40 ANS

DES SCIENCES DE L'ÉDUCATION

L’âge de la maturité? Questions vives
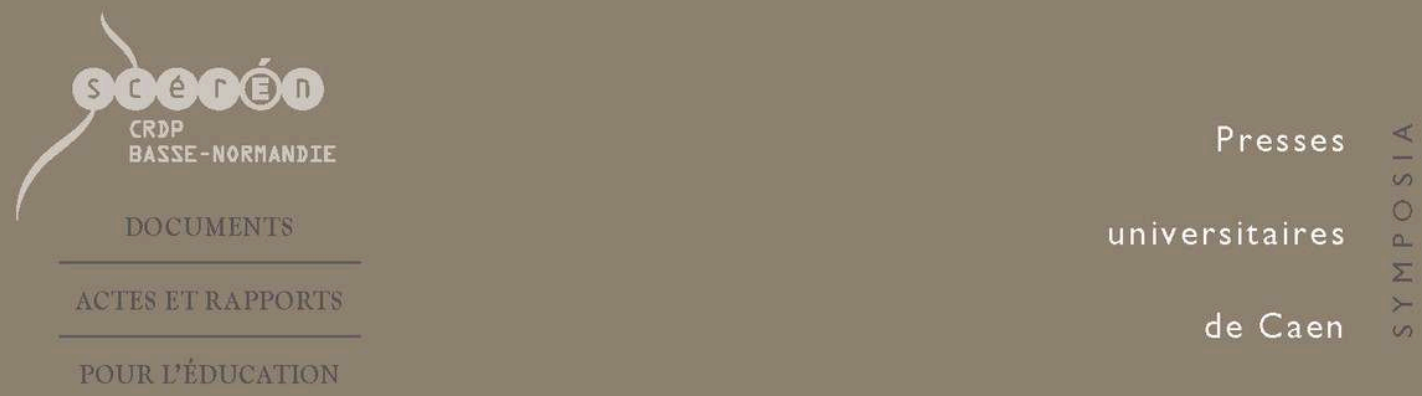
universitaires

de Caen

\section{0 ans des sciences de l'éducation}

L'âge de la maturité ? Questions vives

\section{Alain Vergnioux (dir.)}

DOI : 10.4000/books.puc. 8131

Éditeur : Presses universitaires de Caen, CRDP de Basse-Normandie

Lieu d'édition : Caen

Année d'édition : 2009

Date de mise en ligne : 27 octobre 2016

Collection : Symposia

EAN électronique : 9782841338214

\section{OpenEdition}

\section{Books}

https://books.openedition.org

\section{Édition imprimée}

EAN (Édition imprimée) : 9782841333486

Nombre de pages : 194

\section{Référence électronique}

VERGNIOUX, Alain (dir.). 40 ans des sciences de l'éducation : L'âge de la maturité ? Questions vives. Nouvelle édition [en ligne]. Caen : Presses universitaires de Caen, 2009 (généré le 08 mars 2022). Disponible sur Internet : <http://books.openedition.org/puc/8131>. ISBN : 9782841338214 . DOI : https://doi.org/10.4000/books.puc.8131 


\section{RÉSUMÉS}

En 1967, les " sciences de l'éducation » étaient créées comme discipline universitaire en particulier à Caen sous l'impulsion de Gastion Mialaret. Le colloque organisé 40 ans plus tard se donnait pour projet de faire le point sur un certain nombre de questions institutionnelles et épistémologiques sur la discipline :

- Histoire, sociologie, psychologie et philosophie de l'éducation : fait-on de la recherche de la même façon dans les disciplines éponymes de référence et dans les sciences de l'éducation?

- Dans quelle mesure et selon quelles modalités les sciences de l'éducation ont-elles été amenées à définir de nouveaux territoires ? Ont-elles suscité de nouvelles problématiques de recherche, accompagné de nouvelles demandes sociales?

\section{ALAIN VERGNIOUX (DIR.)}

Alain Vergnioux est agrégé de philosophie, docteur en philosophie, professeur à l'université de Caen, responsable de la revue Télémaque, revue de philosophie de l'éducation (PUC) et directeur d'une collection de philosophie de l'éducation aux éditions Vrin. 


\section{ANS DES SCIENCES DE L’ÉDUCATION L’âge de 1a maturité? Questions vives}
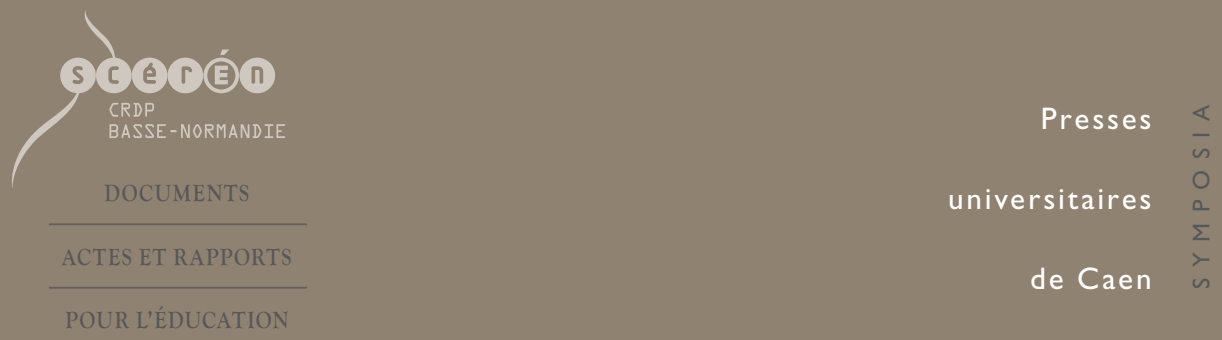


40 ans des sciences de l'éducation

L'âge de la maturité?

Questions vives 
Couverture: @ cliché Éloïse LEGAY

Maquette de Cédric Lacherez

Tous droits de traduction, d'adaptation et de reproduction, sous quelque forme que ce soit, réservés pour tous pays.

ISBN PUC : 978-2-84133-348-6

(C) 2009. Presses universitaires de Caen 14032 Caen Cedex 5 - France
ISBN CRDP : 978-2-86618-579-4 ISSN : $1159-6538$

(c) 2009. CRDP de Basse-Normandie 14070 Caen Cedex 5 - France 


\title{
40 ans des sciences de l'éducation
}

\author{
L'âge de la maturité ? \\ Questions vives
}

Actes du colloque de Caen (20-22 février 2007)

publiés sous la direction d'Alain Vergnioux

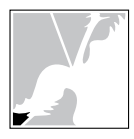

2009

Centre d'études et de Recherches

EN SCIENCES DE L'ÉDUCATION

Université de Caen Basse-Normandie 



\section{OUVERTURE}





\section{LES ORIGINES ET L'ÉVOLUTION DES SCIENCES DE L'ÉDUCATION EN PAYS FRANCOPHONES}

\section{Les préludes de la recherche scientifique en éducation}

La littérature relative à l'éducation est particulièrement abondante. Un de ses trois champs principaux regroupe les essais sur ce que j'appellerai, dès maintenant, les débuts et l'évolution de l'attitude de recherche scientifique sur les questions d'éducation.

Il faudrait remonter très loin dans l'histoire de l'éducation, mais les limites temporelles de cet exposé nous imposent une simplification. Ne citons donc que quelques précurseurs célèbres, accompagnés de quelques citations extraites d'historiens.

Dès le début du XVII ${ }^{\mathrm{e}}$ siècle, J.A. Comenius (1592-1670) évoque implicitement la nécessité d'une pédagogie expérimentale lorsqu'il regrette que le travail scolaire ou universitaire ne fasse pas, comme le travail mécanique, l'objet d'études scientifiques.

Deux siècles plus tard, J.F. Herbart (1776-1841) formule cette exigence d'une manière plus précise lorsqu'il envisage d'annexer une « école expérimentale » à l'Université. En effet, au moment où il est appelé, en 1808, à prendre la succession de Kant à la chaire de philosophie et de pédagogie de Königsberg, il fait part au curateur de l'université d'un projet qui sera, plus tard, repris par Dottrens.

Nous citerons enfin, parmi d'autres, A.-A. Cournot (1801-1877), dont le texte aurait pu être écrit au début du $\mathrm{XX}^{\mathrm{e}}$ siècle. Dans son Essai sur les fondements de nos connaissances, Cournot ouvre une perspective quelque peu nouvelle et n'hésite pas, comme le montre Maurice Debesse, à hisser la pédagogie au rang des disciplines expérimentales. En pédagogie comme en médecine, écrit-il, on n'est pas strictement borné à l'observation des phénomènes tels qu'ils se reproduisent d'eux-mêmes; l'expérimentation directe n'est pas impossible, quoique le respect dû à la nature humaine et le but de l'art ajoutent aux difficultés intrinsèques de l'expérimentation et en restreignent le champ. Afin de surmonter ces difficultés, Cournot préconise l'emploi des statistiques pour étudier, par exemple, les liaisons entre des « aptitudes » différentes ou la relation entre le degré d'instruction et le taux de criminalité. Mieux encore, anticipant l'utilisation des techniques modernes d'analyse factorielle, il se propose de déterminer indirectement quelles sont les facultés entre lesquelles la nature a mis le 
plus d'indépendance par la constitution des organes de pensée, de manière à confirmer ou à renverser une théorie fondée sur des considérations déterministes, comme le système phrénologique de Gal.

\section{Les débuts de la recherche scientifique sur l'éducation}

L'attitude scientifique dans la recherche sur l'éducation a donc une certaine ancienneté et il n'y a que les ignorants (comme malheureusement on en entend actuellement) qui croient que la recherche scientifique sur l'éducation en est encore à l'ère des balbutiements. On voit, en effet, dès la fin du XIX ${ }^{\mathrm{e}}$ siècle et au début du XX $\mathrm{XX}^{\mathrm{e}}$ (avant la guerre de 1914-1918), un essor particulièrement riche en ce domaine.

\section{La période qui précède la guerre de 1914-1918}

En France, c'est Alfred Binet qui en est le chef de file. Dès la fin du XIX ${ }^{\mathrm{e}}$ siècle, ses recherches aussi bien en laboratoire que dans l'école de la rue de la Grange-aux-Belles font de lui un des précurseurs authentiques de la recherche scientifique dans le domaine de l'éducation. Le texte suivant, extrait du livre La fatigue intellectuelle (écrit en collaboration avec V. Henri, en 1898), témoigne de la netteté de ses positions:

La Bibliothèque de pédagogie et de psychologie que nous inaugurons aujourd'hui en publiant ce premier volume sur la fatigue intellectuelle, est destinée à faire profiter la pédagogie des progrès récents de la psychologie expérimentale.

Ce n'est pas, à proprement parler, une réforme de la pédagogie ancienne qu'il faut tenter, mais la création d'une pédagogie nouvelle. L'ancienne pédagogie, malgré de bonnes parties de détail, doit être complètement supprimée, car elle est affectée d'un vice radical: elle a été faite de chic, elle est le résultat d'idées préconçues, elle procède par affirmations gratuites, elle confond les démonstrations rigoureuses avec des citations littéraires, elle tranche les plus graves problèmes en invoquant la pensée d'autorités comme Quintilien et Bossuet, elle remplace les faits par des exhortations et des sermons; le terme qui la caractérise le mieux est celui de verbiage.

La pédagogie nouvelle doit être fondée sur l'observation et sur l'expérience, elle doit être, avant tout, expérimentale. Nous n'entendons pas ici par expérience ce vague impressionnisme des personnes qui ont beaucoup vu; une étude expérimentale, dans l'acception scientifique du mot, est celle qui contient des documents recueillis méthodiquement, et rapportés avec assez de détails et de précision pour qu'on puisse, avec ces documents, recommencer le travail de l'auteur, le vérifier, ou en tirer des conclusions qu'il n'a pas remarquées.

Les expériences de pédagogie psychologique peuvent être divisées en deux groupes: $1^{\circ}$ celles qui sont faites dans les laboratoires de psychologie et $2^{\circ}$ celles qui sont faites dans les écoles.

En pédagogie ce sont surtout les expériences du deuxième groupe qui sont appréciées, mais il ne faut pas négliger pour cette raison les expériences de laboratoire. En effet, dans les laboratoires de psychologie on fait des recherches sur un petit nombre de personnes qui en général viennent au laboratoire pour apprendre la psychologie, et se prêtent par conséquent avec beaucoup de bonne volonté aux expériences. Avec 
ces personnes comme sujets, on peut faire des examens très minutieux, on peut étudier l'influence des différentes causes d'erreur, chercher si telle méthode peut donner quelque résultat ou non, essayer de nouvelles méthodes et les perfectionner de façon à les rendre pratiques et simples ${ }^{1}$.

Binet et Henri terminent leur livre par un vœu qui est encore loin d'être exaucé:

que l'administration française, trop éclairée pour ne pas comprendre l'intérêt supérieur de ces études, se persuade bien qu'on ne résoudra aucun problème pédagogique par des discussions, des discours et des joutes oratoires, et favorise de tout son pouvoir les recherches de psychologie expérimentale dans les écoles ${ }^{2}$.

L' «échelle métrique de l'intelligence» peut être considérée comme la frontière entre l'ancienne et la nouvelle psychologie. Alors que les intellectuels français se moquent de ce «peseur de l'intelligence», le monde entier prend conscience de l'importance de la révolution apportée en psychologie.

On comprend avec quelle faveur fut accueillie l' " échelle métrique de l'intelligence» que Binet publiait en 1905, en collaboration avec Théodore Simon, et qui fournissait le moyen de diagnostiquer, en années, et même en mois, le retard ou l'avance intellectuelle d'un écolier.

Ce travail remarquable, et plein de promesses, passa complètement inaperçu en France. Tout au plus y provoqua-t-il quelques railleries de la part de pédagogues facétieux. Mais à l'étranger, il ne manqua pas de soulever l'admiration générale, puis, un enthousiasme allant jusqu'à l'emballement, aux États-Unis notamment. Les tests de Binet et Simon furent vérifiés à Bruxelles par le $\mathrm{D}^{\mathrm{r}}$ Decroly, à Genève par $\mathrm{M}^{\text {elle }}$ Descœudres, aux États-Unis par Goddard, Kuhlmann et d'autres, en Allemagne par Bobertag, à Stockholm par Jæderrholm, à Milan par Treves et Saffiotti. Partout, abstraction faite de critiques de détail, on les trouva entièrement satisfaisants.

Dès le début du siècle, en effet, Binet se propose d'analyser scientifiquement trois problèmes essentiels: les programmes de l'enseignement, les méthodes d'enseignement, les aptitudes des enfants. Édouard Claparède, à Genève, répondra à ce désir avec son livre, fondamental pour l'époque: Comment diagnostiquer les aptitudes chez les écoliers. C'est à cette époque aussi que Binet, travaillant dans l'école de la rue de la Grange-auxBelles et aidé par le directeur de cette école, Vaney, établit des «barèmes d'instruction ». La question était déjà dans l'air, comme le prouve le court texte suivant:

Tout en signalant le souci, exprimé en 1864 par le Rév. G. Fischer, de mettre en œuvre des standards pédagogiques pour évaluer les travaux scolaires, on voit dans J.M. Rice (18571934), le pionnier de l'organisation scientifique de l'éducation. Il est vrai que J.M. Rice avait fondé en 1903 la première Society of Educational Research et publié, en 1914, son Scientific Management in Education, au moment où F.W. Taylor se proposait d'appliquer au travail industriel ses célèbres Principles of Scientific Management (1911).

1. Mialaret 2006, 20.

2. Ibid., 23 . 
Le barème d'instruction de Vaney, établi pour les écoles primaires de Paris, met en relation des exercices scolaires standardisés (par exemple un problème de calcul simple: "soustraire 8 sous de 59 sous. Combien en reste-t-il? » à un âge moyen de réussite, en l'occurrence 7 à 8 ans).

Dans son commentaire, Binet souligne que la méthode de la comparaison garantit la nature scientifique de la mesure de l'intelligence et la validité des tests. Le principe de la méthode, dit-il, se résume dans les deux propositions suivantes:

- l'examen n'est pas livré au hasard, au caprice de l'inspiration, aux surprises des associations d'idées, il se compose d'un système de questions dont la teneur est invariable et dont la difficulté est dosée;

- le degré d'instruction d'un enfant n'est point jugé in abstracto comme bon, médiocre, mauvais, suivant une échelle subjective de valeurs; il est comparé au degré d'instruction de la moyenne d'enfants de même âge et de même condition sociale qui fréquentent les mêmes écoles.

Le résultat obtenu peut être aussitôt transformé, sans commentaire d'aucune sorte, en une notation qui exprime qu'un enfant est, pour son instruction, régulier, ou qu'il est en avance de six mois, d'un an, de deux ans... ou au contraire, qu'il est en retard de six mois, d'un an et davantage.

À la même époque aussi, et bien que son ouvrage ait été publié longtemps après sa mort, Émile Durkheim montre, dans le cours professé à la Sorbonne dans la chaire de pédagogie, comment on peut, dans une perspective historico-sociologique, étudier l'histoire de l'éducation. Son livre, L'évolution pédagogique en France, est à placer parmi les grands classiques de la littérature pédagogique.

À l'étranger (francophone), la recherche scientifique en éducation se développe dans d'assez bonnes conditions. Sans pouvoir nous étendre sur ce point, signalons quelques grands noms que tous les auditeurs avertis connaissent parfaitement.

En Belgique, c'est le nom d'Ovide Decroly (1871-1932) qui domine. À la fois psychologue-médecin et éducateur (il crée, en 1907, l'École de l'Ermitage), Decroly s'efforce d'introduire l'attitude scientifique dans l'étude de l'enfant et de son éducation. Le petit livre, signé avec R. Buyse, Introduction à la pédagogie quantitative (publié en 1929, trois ans avant sa mort), reprend et utilise les travaux de Mercante, savant argentin de l'université de La Plata qui applique, dès 1893, la statistique à l'étude des problèmes pédagogiques.

En Italie, la personnalité et les ouvrages de Maria Montesori (1870-1952) influencent à la fois la psychologie de l'enfant et la pédagogie du début du siècle. Elle crée, en 1907 , à la fois pour continuer ses recherches sur la psychologie de l'enfant et sur l'éducation, la célèbre Casa dei bambini (la maison des enfants) et publie, en 1909, son ouvrage fondamental sur la méthode de la pédagogie scientifique appliquée à l'éducation des enfants: Il metodo della pedagogia scientifica applicado all'educazione infantile nelle case dei bambini.

En Suisse, l'École de Genève (d'où va émerger Jean Piaget) est très active. Le nom d'Édouard Claparède (1873-1940) domine, à cette époque, l'ensemble des productions 
psychologiques et pédagogiques. Son ouvrage magistral, Psychologie de l'enfant et pédagogie expérimentale, dont la première édition remonte à 1909, a connu un exceptionnel succès et a été, pour beaucoup d'étudiants d'avant la dernière guerre, un véritable livre de chevet. On pourrait en dire autant de son livre qui a ouvert le chemin à la plupart des examens psychologiques de nature métrique: Comment diagnostiquer les aptitudes chez les écoliers, publié en 1923.

La guerre de 1914-1918 interrompt, en France, toutes les recherches dont Binet avait été un brillant initiateur. Le mouvement bouillonnant et fécond de la recherche sur l'éducation de la fin du XIX ${ }^{\mathrm{e}}$ et du début du XX $\mathrm{XX}^{\mathrm{e}}$ siècle y joue «la Belle au bois dormant». Les questions d'éducation et de pédagogie réapparaissent entre les deux guerres, mais la notion de recherche scientifique, telle que l'avaient introduite Binet et ses collaborateurs, reste en sommeil. Le plus bel exemple nous en est donné par le remarquable tome XV de l'Encyclopédie française, consacré à Éducation et Instruction et dirigé par C. Bouglé. Dans l'introduction, Lucien Fèbvre soulève bien la question des rapports de la psychologie et de l'éducation mais la notion de recherche est totalement absente:

Qu'aucune tentative ne se soit produite, ici ou là, pour tirer d'une psychologie conquérante une pédagogie dûment fondée en savoir expérimental: voilà tout de même qui serait bien surprenant! En fait, un peu partout, de semblables pédagogies sont nées... Mais leurs initiatives ont-elles mordu sur la masse ou laissé puissantes les routines coutumières?

Les préoccupations de la remise en route et en ordre des systèmes éducatifs (perturbés sérieusement pendant les hostilités) sont à l'ordre du jour; elles ne trouveront réellement leur satisfaction qu'avec le plan Langevin-Wallon.

\section{La période 1918-1940}

En France, le choc de quatre ans de dure guerre, la remise en route des différents services de l'État, le redémarrage de l'activité économique, etc., ont fait que les problèmes de la recherche scientifique - et surtout en éducation! - sont passés au second plan des préoccupations des responsables. Les mouvements pédagogiques (surtout dans la perspective de la recherche d'une paix durable) occupent le terrain; le mouvement d'Éducation nouvelle tient son congrès international à Calais en 1920. En 1923 sont publiées les Instructions officielles qui déterminent les programmes et les méthodes de l'école primaire. Les soucis de rénovation l'emportent sur les soucis officiels de la recherche scientifique.

Il faut pourtant signaler que d'autres organismes, étrangers à l'Éducation nationale, poursuivaient des recherches qui, plus ou moins directement, intéressaient l'éducation. C'est le cas du Conservatoire des arts et métiers et de son jeune Institut de psychologie; sous la direction de H. Laugier, H. Piéron, $\mathrm{M}^{\mathrm{me}} \mathrm{H}$. Piéron, du $\mathrm{D}^{\mathrm{r}}$ Toulouse, de $\mathrm{M}^{\text {elle }} \mathrm{D}$. Weinberg, une importante enquête est organisée sur la correction 
des copies du baccalauréat; c'est, à notre sens, un des premiers travaux d'envergure de docimologie. Ce pavé lancé dans la mare de notre saint et intouchable baccalauréat reste sans effet; il faudra attendre la fin de la Seconde Guerre mondiale pour que la question ressurgisse dans les préoccupations des chercheurs en sciences de l'éducation (G. Mialaret, G. de Landsheere, A. Bonboir...).

À l'étranger francophone, afin de ne pas alourdir l'exposé, ne citons que les ouvrages belges suivants qui nous paraissent fondamentaux. L'un est précurseur: O. Decroly et R. Buyse, Introduction à la pédagogie quantitative; l'autre de plus grande envergure scientifique: R. Buyse, L'expérimentation en pédagogie. Le livre de T. Jonckheere, La méthode scientifique en pédagogie, et celui d'É. Planchard, L'investigation pédagogique: objet, méthodes, résultats, font partie des bibliothèques de tout chercheur. Les fondements de la recherche scientifique en éducation sont donc posés; il faudra attendre, après la pose de cette première pierre, plusieurs décennies pour que la recherche scientifique en éducation prenne réellement son essor.

\section{Depuis la fin de la Seconde Guerre mondiale jusqu'en 1953}

Les dures périodes de la guerre et de la Résistance ne laissent pas de place aux préoccupations des chercheurs scientifiques. En 1944, la France est à reconstruire, son économie à remettre en marche, ses structures administratives à réorganiser, la paix sociale à retrouver. Les questions d'éducation étaient pourtant déjà à l'étude, comme nous l'indique le plan d'Alger pour la rénovation de l'éducation. Dès la Libération, la question de la réforme de l'enseignement est à l'ordre du jour ; les mouvements pédagogiques dits d'Éducation nouvelle se multiplient et se développent (GFEN, mouvements Freinet, Cousinet, Cemea...). Au plan national, la Commission de réforme de l'enseignement voit le jour sous la présidence de Paul Langevin et donnera lieu au célèbre plan Langevin-Wallon. Ce travail, par ailleurs très remarquable sur le plan pédagogique, ne consacre pourtant aucun chapitre ou paragraphe à la recherche scientifique en éducation. Toutes les préoccupations, aussi bien des «politiques» que des "praticiens» de l'époque, sont tournées vers les problèmes d'organisation, d'innovation, d'adaptation du service de l'Éducation nationale aux nouvelles conditions de la vie sociale, de la nouvelle et naissante civilisation.

\section{La pédagogie expérimentale}

Pourtant un petit courant, plus ou moins clandestin et largement minoritaire, continue à tracer son chemin: plusieurs recherches se regroupent sous le titre général de "pédagogie expérimentale». Il est nécessaire de s'arrêter un instant sur cette expression dont le véritable sens a donné lieu à de nombreux malentendus. Le mouvement de recherches, inauguré et développé par Alfred Binet au début du XX $\mathrm{XX}^{\mathrm{e}}$ siècle, ne s'est pas totalement évanoui. Dans tous les pays francophones, on peut repérer soit des initiatives individuelles, soit des activités de petits centres de recherche qui, avec leurs petits moyens (quand ils existent!), s'efforcent de développer des projets de recherche. 
En Belgique, il faut signaler le centre de Morlanwelz (avec l'action efficace de F. Hotyat), à Bruxelles, Jonckheere, puis Vandevelde, à Liège Clausse puis Bayer, Crahay, de Landsheere à Gand, Verbist, puis M.L. van Herrewegue, à Louvain, Buyse, Frankard, Gilles, Bonboir, Andréa Jadouille à Angleur et Gérard Goossens à ForestBruxelles. Nous rattacherons à la Belgique le professeur belge Planchard, enseignant à l'université de Coïmbra au Portugal.

En Suisse, va dominer, à Genève, la personnalité de Robert Dottrens puis celle de Samuel Roller, directeur du laboratoire de pédagogie expérimentale de l'Institut des sciences de l'éducation de Genève, tous deux aux côtés de Jean Piaget.

En France seront créés, en 1956, le Centre de recherche de l'Institut pédagogique national (ancien Musée pédagogique), dirigé par Roger Gal, le laboratoire de psychopédagogie de l'École normale supérieure de Saint-Cloud, créé et dirigé par Gaston Mialaret (en 1948). Il faut aussi noter le travail de recherche scientifique très important fait par l'équipe des jeunes psychologues scolaires dirigée par René Zazzo (mise au point de tests de connaissances en particulier).

Toutes les recherches entreprises dans ces lieux s'efforcent d'être scientifiques et, sous l'influence de R. Dottrens, se regroupent sous la bannière de la pédagogie expérimentale. L'expression a, à cette époque, une signification que nous devons rapidement expliquer. La $11^{\mathrm{e}}$ édition du livre de Claude Bernard, Introduction à l'étude de la médecine expérimentale, paraît en 1939; la révolution psychologique est en marche et l'on parle couramment de la psychologie expérimentale. Toutes les activités qui vont rompre avec l'ancienne attitude plus ou moins philosophico-littéraire et qui adoptent une attitude scientifique vont s'attacher le qualitatif d'expérimental. Ce qui va d'ailleurs prêter à confusion parce que, comme nous allons le voir, toutes les disciplines qui vont se regrouper sous la famille des sciences de l'éducation ne sont pas de nature expérimentale.

En 1953 (d'où le choix de cette limite), Robert Dottrens, avec la collaboration de l'université de Lyon, organise la toute première réunion d'une poignée de chercheurs (à peine dix!) qui se revendiquent de la pédagogie expérimentale. Le mouvement est lancé; pendant cinq ans, ce groupe de chercheurs se réunit annuellement pour que les membres puissent se mettre au courant réciproquement de leurs travaux, de leurs préoccupations, de leurs projets et, en 1958, est officiellement créée l'Association internationale de pédagogie expérimentale de langue française (AIPELF), dont la présidence est confiée à Gaston Mialaret. Cette AIPELF va jouer un rôle important dans le développement des recherches scientifiques en éducation et dans la formation des jeunes chercheurs ${ }^{3}$.

\section{La renaissance du courant des sciences de l'éducation}

Dès 1912, était créé, à Genève, un Institut des sciences de l'éducation. Quelques universités étrangères, aux environs de 1960, transforment les collèges et les facultés d'éducation en facultés de sciences d'éducation; le nombre est pourtant limité. En 1960,

3. Mialaret 2006,39 . 
on ne parle encore que très peu de sciences de l'éducation. Une dispute s'était élevée au début du $\mathrm{XX}^{\mathrm{e}}$ siècle: fallait-il parler de science de l'éducation ou de sciences de l'éducation? Ces discussions ont été interrompues par la guerre. Depuis longtemps, par ailleurs, Durkheim avait parfaitement posé le problème de la distinction entre la pédagogie et la recherche scientifique (voir ci-après). Les conditions internationales et la création des grandes organisations internationales (ONU, UNESCO, UNICEF, Conseil de l'Europe...) transforment le paysage culturel; les moyens de communication opèrent leur mutation avec l'apparition de l'audiovisuel, de l'informatique, le développement gigantesque des méthodes et techniques de l'information (publication des Annuaires de l'éducation par l'UNESCO); le développement de nouveaux moyens de communication ouvrent une ère nouvelle à l'Humanité. Les préoccupations relatives au rôle de l'éducation et ses relations avec la vie sociale, technique et économique, dans les pays en voie de développement, la nécessité de faire un extraordinaire effort d'alphabétisation, font que les préoccupations relatives à l'éducation prennent une place de premier plan et font appel à de nouvelles disciplines scientifiques: sociologie, ethnologie, démographie, économie... De plus en plus, on voit l'expression «sciences de l'éducation » se substituer à «pédagogie expérimentale» et le nombre de disciplines scientifiques qui vont se préoccuper de l'éducation devient de plus en plus important.

Un autre mouvement se développe pour introduire un cursus nouveau au niveau des universités et des centres de formation des enseignants. Au début du siècle précédent, Durkheim avait brillamment occupé une chaire de pédagogie à la Sorbonne (chaire créée en 1887 et précédemment occupée par Marion, puis par Ferdinand Buisson); en 1904, on republiait la $6^{\mathrm{e}}$ édition (la première date de 1883) du monumental ouvrage de G. Compayré, professeur à la Faculté des Lettres de l'université de Toulouse: Histoire critique des doctrines de l'éducation en France depuis le XVI siècle; Chabot et Jean Bourjade assuraient un enseignement de pédagogie à l'université de Lyon; en 1911, le professeur Lebonnois, de l'université de Caen, créait un Institut pédagogique international. L'Université française n'était donc pas totalement étrangère aux préoccupations pédagogiques. Mais, entre les deux guerres, le bastion principal de la pédagogie est constitué par les Écoles normales de l'enseignement primaire. L'idée d'un enseignement de la pédagogie au niveau universitaire et le besoin de l'organisation d'une recherche scientifique en éducation ne sont développés que par quelques enseignants du supérieur. Sous l'influence des nouvelles réalités et contraintes internationales, sous les effets des actions menées par certains universitaires convaincus (M. Debesse, J. Chateau, G. Mialaret en particulier) et de quelques personnalités éminentes de l'époque (J. Stoetzel et P. Fraisse), les autorités ministérielles françaises se décident, enfin, à créer un cursus universitaire de sciences de l'éducation ${ }^{4}$. C'est ce que nous avons appelé «le coup de tonnerre du 30 novembre 1966 ». C'est ainsi qu'à la rentrée universitaire de 1967, trois universités françaises: Bordeaux, Caen et Paris, organisèrent, au sein des facultés des Lettres, un cursus de sciences de l'éducation.

4. Voir Mialaret 2006, $46 s q$. 


\section{L'histoire et l'enrichissement du concept de «sciences de l'éducation»}

L'expression «sciences de l'éducation" est relativement récente dans la langue française; des ouvrages importants tels que l'Encyclopedia universalis, publiée en 1968, le Dictionnaire de la langue pédagogique publié en 1971 par P. Foulquié, ne la mentionnent pas encore. Utilisée en 1912 à Genève, elle ne recouvre qu'un domaine très limité de l'éducation: psychologie de l'enfant, enfants difficiles ou anormaux, formation des enseignants; à cela on peut ajouter la philosophie et l'histoire des doctrines pédagogiques. Les premiers projets présentés après la réunion qui s'est tenue au ministère de l'Éducation nationale en 1966, quel que soit leur intérêt intrinsèque, témoignent de la pauvreté relative du concept de sciences de l'éducation; ce n'est que sous diverses influences que va s'enrichir ce concept actuellement accepté.

\section{La distinction entre la pédagogie et les sciences de l'éducation: une autre attitude épistémologique}

Depuis longtemps, la distinction couvait dans la pensée scientifique. Dans un célèbre passage d'Éducation et Sociologie, Durkheim précisait bien la distinction à faire entre deux domaines: celui de la science et celui de la pédagogie. Dans le premier cas, «il s'agit simplement ou de décrire des choses présentes ou passées, ou d'en rechercher les causes, ou d'en déterminer les effets». Il poursuit:

Les théories que l'on appelle pédagogiques sont des spéculations d'une tout autre sorte. En effet, ni elles ne poursuivent le même but, ni elles n'emploient les mêmes méthodes. Leur objectif n'est pas de décrire ou d'expliquer ce qui est ou ce qui a été, mais de déterminer ce qui doit être. Elles ne sont orientées ni vers le présent, ni vers le passé, mais vers l'avenir. Elles ne proposent pas d'exprimer fidèlement des réalités données, mais d'édicter des préceptes de conduite. Elles ne nous disent pas: voilà ce qui existe et quel en est le pourquoi, mais voilà ce qu'il faut faire ${ }^{5}$.

À la suite de la sociologie (en particulier l'influence de la position épistémologique de Durkheim), de la psychologie qui «de la science de l'âme» devient «la science des conduites" (toute l'École psychologique contemporaine), les «sciences de l'éducation " opèrent leur tournant épistémologique en étudiant, dans une perspective scientifique, les situations d'éducation. Les sciences de l'éducation sont du domaine de l'observation, du descriptif et de l'explication; la pédagogie est du domaine du prescriptif et de l'action éducative, sans aucune idée sous-jacente de hiérarchie entre les deux domaines.

Les sciences de l'éducation sont constituées par l'ensemble des disciplines scientifiques qui étudient, dans des perspectives différentes mais complémentaires et coordonnées, les conditions d'existence, de fonctionnement et d'évolution des situations et des faits d'éducation ${ }^{6}$.

5. Durkheim 1934, 75.

6. Mialaret 2006, 69. 


\section{Une nouvelle réflexion sur le concept d'éducation}

Le classique modèle socratique est largement dépassé; la relation éducative ne se ramène plus - ou seulement dans quelques cas particuliers - à la mise en présence d'un maître et d'un disciple. Le concept d'éducation s'est considérablement enrichi aussi bien du point de vue compréhensif qu'extensif. L'éducation, sous des formes et des modalités différentes, va de la naissance (et même avant!) jusqu'à la mort; elle s'intéresse à tous les aspects de la personnalité du sujet ou aux différentes caractéristiques du groupe. L'éducation d'un sujet n'est plus uniquement celle qu'il reçoit au sein de l'institution scolaire, mais aussi au sein de cette «école parallèle» dont parle G. Friedmann; l'éducation actuelle ne peut plus se ramener au modèle enseignantenseigné.

Les techniques modernes (audiovisuel, informatique, presse...) donnent un visage particulier aux situations d'éducation.

Une situation d'éducation est une situation sociale dynamique traversée par des finalités plus ou moins clairement explicitées, et composée de sujets n'ayant pas tous, ni le même statut social, ni le même rôle à tenir. Une situation d'éducation se déroule dans un lieu et dans un temps définis; elle a une histoire; souvent, elle s'ouvre sur le futur.

Une situation d'éducation comporte (selon les cas) un certain nombre d'organismes et de services qui participent à l'action éducative telle qu'elle est prévue dans les finalités et les modalités de l'action éducative envisagée: services de santé, services psychologiques et de rééducation, services de documentation, services d'évaluation, services sociaux, services de recherche, services techniques d'audiovisuel et d'informatique.

Les actions déterminantes de l'éducation font par ailleurs intervenir, dans des sphères plus ou moins proches, le milieu familial et les communautés de voisinage, l'environnement social et le mode du travail, l'expérience personnelle et de façon interne les recherches pédagogiques et théoriques sur les questions d'éducation et de formation.

Tous les travaux actuels, et cela dans toutes les disciplines scientifiques, ont mis en évidence la complexité du déterminisme des situations d'éducation, de l'éducation elle-même.

\section{Quelques aspects épistémologiques}

\section{Connaissance empirique et connaissance scientifique}

Ce qui porte la démarche du praticien se trouve au sein de l'action, par intuition le plus souvent, dans la confrontation à de nouvelles situations, à la recherche de nouvelles façons d'intervenir et d'agir: les connaissances auxquelles il parvient sont d'ordre «praxéologique». 
Le chercheur en sciences de l'éducation met en œuvre les méthodes de l'investigation scientifique et vise des résultats de nature scientifique; il vise ensuite à tirer de ses recherches des conclusions pratiques qu'il propose au praticien.

Il ne s'agit pas d'établir une relation hiérarchique entre les deux formes de savoir; elles sont d'ordre différent, elles ne sont pas contradictoires mais doivent collaborer à la constitution du savoir en éducation. Le praticien reste toujours le responsable de son action éducative, aussi bien dans le choix de ses finalités que dans celui de ses méthodes et techniques d'application.

\section{Les domaines d'étude}

La réflexion sur le concept d'éducation a montré la polysémie du terme. Il est donc normal de retrouver les quatre domaines d'étude qui exigent, pour être analysés, de faire appel à des techniques scientifiques quelquefois différentes:

- l'éducation considérée sous l'angle de ses aspects administratifs et réglementaires: l'éducation-institution (aspects législatifs);

- l'éducation considérée sous l'angle des contenus: programmes, matières (aspects scientifiques, besoins de la société);

- l'éducation considérée sous l'angle de ses résultats, des types de formés qui sortent du système scolaire (aspects psychologiques);

- l'éducation sous l'angle de la pratique concrète, de l'action exercée en vue d'atteindre les objectifs définis (aspects pédagogiques).

\section{Trois types de situation d'étude}

On peut considérer les domaines d'étude sous un autre angle: celui de la stabilité des situations. On peut distinguer trois formes principales:

- les situations du passé sur lesquelles nous n'avons plus aucune possibilité d'action, exemple: l'éducation en Grèce. L'étude ne peut se faire qu'à partir de documents (textes, œuvres d'art...): situations définitivement stables;

- les situations relativement stables dans le temps: elles sont contemporaines du chercheur et d'une durée de vie plus ou moins longue (exemple: l'état du système universitaire français en 2007). Il est possible d'analyser les textes qui organisent le système; on peut interroger soit les personnes qui ont été à l'origine des textes, soit les personnes qui les appliquent et les mettent en œuvre (enseignants, corps d'inspection, parents d'élèves, hommes politiques), soit les organismes qui reçoivent les étudiants en fin d'études (entreprises), soit les personnes qui font fonctionner le système (administrateurs, enseignants, étudiants, services de santé, services de psychologie et d'orientation...);

- les situations essentiellement dynamiques qui sont celles au cours desquelles s'exerce l'action éducative. Elles sont limitées dans le temps et dans l'espace, uniques et non reproductibles à l'identique. 


\section{Les types de connaissances}

À la variété des domaines d'étude, des types de situation d'éducation correspondent, évidemment, des types de connaissances variés. En voici quelques-uns:

- les connaissances de type psychologique ou de témoignage. Ce sont celles que l'on recueille soit par observation, soit par entretien; elles résultent de l'action du chercheur et doivent être soumises à une très précise analyse critique pour en assurer un degré d'objectivité compatible avec une recherche scientifique;

- les connaissances de type réflexif, philosophique. C'est ici tout le domaine de la philosophie de l'éducation, qui porte aussi bien sur les doctrines, les finalités que sur les méthodes et techniques utilisées;

- les connaissances de type historique et l'établissement des faits. Nous sommes ici dans le domaine de l'historien qui va procéder, selon ses habitudes, à la critique interne et externe des documents, pour en apprécier le degré d'objectivité et en rechercher la signification réelle en fonction de la datation du document;

- les connaissances de type taxonomique ou de diagnostic. L'activité de recherche est analogue à celle du naturaliste qui s'efforce de construire des tables de classification afin de pouvoir émettre un diagnostic. Comment classer les différentes méthodes d'apprentissage de la lecture et que signifie exactement méthode syllabique ou méthode globale?

- les connaissances de type expérimental. Ce sont les connaissances qui résultent de l'application stricte d'un plan expérimental à des faits ou à des situations d'éducation. La difficulté principale tient au fait que le nombre des variables indépendantes et dépendantes est pratiquement infini, et le chercheur doit obligatoirement faire un choix pour pouvoir gérer la situation dans de bonnes conditions;

- les connaissances de type statistique. Toutes les enquêtes, l'application de questionnaires (quand ils sont construits selon les règles de la méthode scientifique!), toutes les statistiques, officielles ou non (classification des lycées en fonction des résultats au baccalauréat, par exemple), fournissent aux chercheurs une masse impressionnante d'informations que ceux-ci doivent ensuite classer, analyser, interpréter.

\section{Les sources du savoir en sciences de l'éducation}

Les sources du savoir en sciences de l'éducation sont nombreuses, variées, hétérogènes. On peut en repérer quatre principales:

- la pratique pédagogique (pratique directe, pratique observée, pratique partagée);

- les services de documentation (nationaux ou internationaux);

- l'ensemble des résultats de recherches qui relèvent des sciences de l'éducation;

- l'ensemble des résultats des travaux, réflexions et discussions d'ordre philosophique, historique, politique. 


\section{Conclusion}

Nous devons prendre conscience qu'un choix fondamental est à faire et nous retrouvons ici la fausse distinction de Dilthey entre l'explication et la compréhension. Je dis «fausse » parce que le mot est, tout d'abord, mal choisi. Ensuite l'explication n'est pas l'opposé de la compréhension : les deux attitudes ne se situent pas au même moment dans la chaîne de la recherche scientifique. L'option «explication» ne peut pas rendre compte totalement de la réalité éducative, et la «compréhension» (au sens de Dilthey) peut aider à la recherche de nouvelles hypothèses de travail, ou à fournir, en attendant des vérifications ultérieures, des interprétations des résultats obtenus qui ne peuvent, dans l'état actuel, être parfaitement confirmés par la recherche scientifique; compréhension et explication contribuent donc ensemble à la constitution du savoir en éducation. Le problème fondamental, pour le chercheur, est donc de savoir faire lucidement la distinction entre ce qui relève de la «compréhension» et ce qui relève de «l'explication», pour donner aux deux séries de données leur propre statut épistémologique et ne pas tout mélanger dans le compte rendu de la recherche.

Permettez-moi, en terminant cet exposé, de vous dire le plaisir que j'ai eu à prendre la parole dans cette université où j'ai passé l'essentiel de ma carrière et à laquelle je suis resté très attaché. Les sciences de l'éducation - que nous avons eu tant de mal à faire naître et à développer - sont, aujourd'hui, entre les mains d'une nouvelle et jeune génération. J'ai une grande confiance en cette jeune génération, en son enthousiasme et sa volonté de pousser encore plus loin le développement de nos sciences de l'éducation, dont l'évolution n'est certainement pas terminée. Alors bon courage et bonne route à tous.

Gaston Mialaret

Professeur d'université honoraire

\section{Références bibliographiques}

Bernard C. (1865), Introduction à la médecine expérimentale, Paris, Delagrave.

Binet A., Henri V. (1898), La fatigue intellectuelle, Paris, Schleicher frères.

Bouglé C. (dir.) (1939), Éducation et instruction (tome XV de l'Encyclopédie française), Paris, Société de gestion de l'Encyclopédie française.

Buyse R. (1935), L'expérimentation en pédagogie, Bruxelles, Lamertin.

Claparède É. (1923), Comment diagnostiquer les aptitudes chez les écoliers, Paris, Flammarion. 
Decroly O., Buyse R. (1929), Introduction à la pédagogie quantitative. Éléments de statistiques appliqués aux problèmes pédagogiques, Bruxelles, Lamertin.

Durкheim É. (1934), Éducation et sociologie, Paris, Alcan.

Durкheim É. (1938), L'évolution pédagogique en France, Paris, Alcan.

FoulquiÉ P. (1971), Dictionnaire de la langue pédagogique, Paris, PUF (Quadrige).

Mialaret G. (1976), Les sciences de l'éducation, Paris, PUF (Que sais-je?).

Mialaret G. (2006), Sciences de l'éducation: aspects historiques, problèmes épistémologiques, Paris, PUF (Quadrige). 


\section{DE LA PSYCHOPÉDAGOGIE À LA RECHERCHE ÉDUCATIONNELLE}





\section{PRÉSENTATION : ÉVOLUTION DES QUESTIONS}

1967-2007: depuis quarante ans, la question de la pédagogie est de manière récurrente une question vive qui se pose aux sciences de l'éducation au cours de leur développement. Et force est de prendre acte que les réponses qui ont été formulées continuent à alimenter la controverse scientifique, au meilleur sens du terme.

Des quatre disciplines fondatrices des sciences de l'éducation, dans leur acception moderne en 1967, la psychologie, peut-être plus encore que l'histoire, la philosophie ou la sociologie, a une place privilégiée au sein du débat. Un débat qui prend plusieurs formes: sur le plan épistémologique se pose la question de la place de la psychologie parmi les sciences de l'éducation, alors que la psychologie, en tant que discipline universitaire, marque un intérêt soutenu et jamais démenti sur les apprentissages de l'individu. Cette question fait l'objet de plusieurs contributions dans les actes de ce colloque, mais la question se pose également d'un point de vue historique avec l'évolution de la psychopédagogie originelle et ses ambitions vers la recherche éducationnelle (néologisme emprunté à nos collègues québécois); elle se pose également d'un point de vue international, au regard notamment des recherches anglophones en éducation, dont il convient d'emblée de remarquer qu'elles concourent pour près de $90 \%$ à la production scientifique mondiale; elle se pose enfin dans la tension historique de la pédagogie avec les didactiques des disciplines, tension vivante dont le débat entre les pédagogues et les républicains peut être pensé comme un développement.

Historiquement, comme le développera la contribution de Marguerite Altet, la psychopédagogie est issue de la psychologie d'inspiration béhavioriste. Celle-ci emprunte ses méthodes de recherche au modèle expérimental des sciences dures auxquelles elle aspire à ressembler. C'est le binôme classique stimulus / réponse qui sert de référence quasi exclusive dans les années 1950-1960.

Tout en s'écartant des apories de la psychopédagogie expérimentale, les recherches processus / produit restent, comme celle-ci, fortement ancrées dans une épistémologie néo-béhavioriste ${ }^{1}$ et largement prescriptive. Elles fondent explicitement leur existence sur la recherche rationnelle des facteurs d'efficacité de l'enseignement ${ }^{2}$.

1. Durant 1996, chap. 1.

2. Walberg 1986. 
Elles ont pour ambition de rendre compte de régularités systématiques entre, d'une part, les résultats observables et quantifiables de l'action pédagogique (les produits) et, d'autre part, les processus d'enseignement. Elles portent, par exemple, sur les connaissances scolaires des élèves, leur vitesse d'acquisition, leurs performances pour mémoriser, leur niveau de compréhension... à travers des tests normalisés, ainsi que sur les comportements et attitudes des élèves: motivation, engagement dans la tâche, autonomie, coopération / compétition, attention cognitive... Au rang des variables processus, on peut proposer, sans prétendre dresser un catalogue : les comportements des enseignants, les caractéristiques récurrentes de leurs pratiques (fréquences et caractéristiques des reformulations, usage des exemples et métaphores, type et nature des tâches scolaires proposées...), les conditions d'utilisation de matériels pédagogiques, diverses variables d'organisation pédagogique...

Ces recherches, menées en grand nombre dans les années 1970 à 1980, s'intéressent à une multiplicité de variables, et l'ouvrage de M. Postic, intitulé Observation et formation des enseignants ${ }^{3}$, en présente une synthèse érudite ainsi que leur utilisation possible dans la conduite de situations d'enseignement ou de formation. Sur le plan de la démarche scientifique, il s'agit:

- (1) de sélectionner des groupes d'élèves ayant des résultats contrastés dans un domaine de performance (produit final);

- (2) de faire l'hypothèse de l'influence d'une variable du processus pédagogique de transformation appelé processus d'enseignement-apprentissage: clarté de la consigne, modalités de régulation interactive, dispositif pédagogique formalisé...;

- (3) de mener des observations instrumentées rigoureuses qui décrivent et relèvent les informations sélectionnées, c'est-à-dire qui permettent de coder en les quantifiant les différentes positions ou valeurs de la variable retenue;

- (4) de réaliser des calculs statistiques ${ }^{4}$ plus ou moins élaborés de corrélation (analyses multivariées, modèles de régression...) entre les différentes modalités du processus étudié et les performances obtenues;

- (5) de caractériser à partir de l'intensité de la corrélation calculée un lien causal entre processus et produit.

Un prolongement pragmatique des résultats obtenus en formation consiste à entraîner les enseignants à développer les comportements attestés comme efficaces pour qu'ils les systématisent dans leurs pratiques pédagogiques et les transforment par habitude en des ressources pédagogiques ordinaires.

On mesure l'intérêt pratique et fonctionnel d'une telle posture de recherche à un moment où les sciences de l'éducation sont en cours de stabilisation et en recherche de légitimation. L'accumulation de résultats ainsi obtenus permet d'abonder un répertoire des «pratiques enseignantes efficaces» qui constituent un atout précieux pour professionnaliser un métier longtemps tenu comme lié, non pas à des gestes

\footnotetext{
3. Postic 1977.

4. Mialaret 1991.
} 
professionnels identifiables et reproductibles et potentiellement évaluables ${ }^{5}$, mais à des qualités intrinsèquement attachées à l'intimité quasi privée de l'enseignant et d'essence vocationnelle: avoir l'amour des enfants, avoir le sens pédagogique.

Cependant, une critique radicale leur est souvent opposée, qui affaiblit considérablement leur intérêt à la fois sur le plan scientifique et sur le plan pratique, plans sur lesquels pourtant elles ont apporté des contributions significatives. Ces travaux ne s'adossent à aucune théorie d'ensemble et n'ont pas participé à produire des éléments théoriques susceptibles de proposer un cadre d'ensemble clair aux résultats qu'ils ont produits. De fait, M. Durand les qualifie d'a-théoriques ${ }^{6}$. Sur le plan scientifique, l'ensemble de ces travaux apparaît plus sous forme de capitalisation de résultats locaux et en même temps relativement décontextualisés. Cette parcellisation est le prix élevé payé par un attachement peu revendiqué et peu élucidé, et même souvent implicite, au paradigme béhavioriste: les corrélations statistiques des variables mises en relation fournissent peu d'informations qui dépassent la dimension comportementale de l'activité de l'enseignant. L'attribution d'un lien causal entre processus et produit est difficilement falsifiable, puisqu'aucune théorie n'encadre les conclusions auxquelles on parvient, ni n'autorise d'interprétation concurrente. L'approche statistique corrélationnelle issue des observables de la situation pédagogique postule, plus qu'elle ne démontre, un lien causal entre processus et produit. De plus, cette approche suppose toujours implicitement que les résultats d'apprentissage sont obtenus à travers une répétitivité, un caractère cyclique des comportements enseignants et des situations pédagogiques auxquels sont confrontés les élèves, et ignorent l'événementiel (accidit) ou apprentissage par insight, qui peuvent, comme tout apprenant en a fait l'expérience, permettre d'accéder à une compréhension et un apprentissage.

Finalement, ces résultats ne permettent pas de comprendre ce qui fonde les comportements des enseignants, d'où leur faible valeur sur le plan strictement scientifique. Ils contournent «la boîte noire» des béhavioristes et ouvrent sur une dimension de la pédagogie essentiellement programmatique, organisationnelle et comportementaliste. Les questions auxquelles ne répondent pas les recherches processus-produit touchent à la structure profonde des situations d'enseignement-apprentissage, à l'économie interne de ces situations à la fois récurrentes et toujours singulières. Elles concernent finalement l'intelligence interne des pratiques enseignantes, si on tient les enseignants non pas pour des agents pédagogiques mais pour des acteurs, et si on postule que les comportements de ces acteurs, loin d'être référés de manière exhaustive à des normes ou standards externes, sont aussi l'expression de processus concernant la perception et les décisions qui sont instanciées dans la pratique.

Les sciences cognitives vont alors aborder l'impensé de la psychologie comportementaliste et s'intéresser à «la boîte noire», provoquant du même coup un déchirement au sein de la psychologie, entre les courants cliniques, qui eurent un succès

5. On peut imaginer des « référentiels qualité» des comportements professionnels des enseignants pour servir de grilles d'évaluation d'une évaluation «au mérite».

6. Durand 1996. 
important dans les années 1970 et 1980, et les courants néopositivistes d'inspiration cognitive, qui vont eux-mêmes être l'objet de querelles entre les tenants d'une neuropsychologie qui se rapproche de la médecine et une psychologie qui tente de sauver son autonomie centrée sur la compréhension des événements et faits psychiques. On peut signaler que ces tensions existent tant dans l'espace francophone que dans le monde anglo-saxon: ce dernier, contrairement à une idée communément admise, ne se réduit pas, loin s'en faut, au paradigme cognitiviste «pur» tel qu'exposé dans les travaux de J. Fodor sur la philosophie de l'esprit ${ }^{7}$ ou bien ceux de S. Pinker sur l'inscription biologique de l'esprit ${ }^{8}$.

La recherche éducationnelle va prendre son essor dans les années 1990, en France mais aussi au Québec, autour de la notion de situations d'enseignement-apprentissage qui placent au premier plan des interactions entre les acteurs individuels, sociaux, institutionnels, les contextes et les dynamiques qui émergent, à la fois récurrentes et contingentes. La proximité que la psychopédagogie originelle entretenait avec la psychologie classique s'est effacée au profit d'une convergence épistémologique entre la recherche éducationnelle, qui n'est pas toujours éloignée d'une certaine forme de néopositivisme, et les sciences de l'action, voire de l'ergonomie cognitive et la didactique professionnelle ${ }^{9}$. En complément des gestes observables, qui peuvent être décrits, on s'intéresse désormais à la dimension descriptible de l'activité des enseignants, c'est-à-dire à la pensée des enseignants (research on teacher's thinking) : les informations qu'ils perçoivent et sélectionnent, les matrices d'interprétations du réel, les arbitrages et la prise de décision, les automatismes, qui commandent l'action, prise dans une tension entre un déterminisme pluriel (situation de départ de l'interaction en classe), une finalisation de cette action (prescriptions institutionnelles et interprétations de ces prescriptions par les enseignants dans des contextes particuliers et contingents), émergence d'une réalité complexe et en partie inédite dans le cours d'action des situations d'enseignement-apprentissage elles-mêmes. Ce paradigme de la recherche éducationnelle va s'accompagner d'un renouveau sur le plan méthodologique: par exemple les entretiens par autoconfrontation des acteurs avec les enregistrements vidéo-numériques de leurs activités permettent d'explorer de manière fructueuse les informations sur la dimension cognitive de l'activité et des pratiques des enseignants mais aussi des élèves ${ }^{10}$.

La question de la tension entre didactique et pédagogie a connu au cours de ces quarante années des conflits autant épistémologiques que des luttes concernant le territoire symbolique de l'enseignement et la formation, des malentendus mais aussi des tentatives d'élaboration commune, comme le montre la problématisation proposée par Gérard Sensevy. Rappelons que si la pédagogie est ancienne, les didactiques disciplinaires, d'abord en mathématiques puis en français, naissent dans les années 1970.

\footnotetext{
7. Fodor 1986.

8. Pinker 1999.

9. Vergnaud et al. 2006.

10. Piot 2008 .
} 
Si l'on accepte de ne pas penser séparées d'un côté la pédagogie, plus centrée sur les dimensions relationnelles et transversales de l'action de l'enseignant pour favoriser les apprentissages des élèves, et de l'autre côté la didactique, soucieuse du traitement des informations, de la structuration des savoirs, il faut alors affronter la question de leur imbrication dans l'ordinaire des situations de classe, sans prétention hégémonique de l'un ou l'autre point de vue ${ }^{11}$. L'entrée par l'analyse de l'activité des enseignants et par l'observation des organisateurs des pratiques ouvre des perspectives fructueuses de questionnement croisé qui s'accompagnent d'un renouveau des problématiques respectives de la pédagogie et de la didactique, tout en trouvant un cadre théorique dans les théories de l'action et de l'interactivité des acteurs que sont les enseignants (pilotes des situations) et les élèves (objets et sujets des transformations visées).

En conclusion, on peut noter qu'en quelques décennies les problématiques autour de la recherche éducationnelle (la pédagogie, la didactique, les situations d'enseignementapprentissages et les activités réciproques, distinctes et intimement reliées de l'enseignant et des élèves) ont été l'objet de débats scientifiques denses, au moment même où, avec l'avènement de "la société de la connaissance», ces questions, en même temps qu'elles acquièrent une actualité forte, requièrent, tant sur le plan épistémologique que méthodologique, d'être abordées avec lucidité.

Thierry Piot

CERSE

Université de Caen-Basse-Normandie

\section{Références bibliographiques}

Durand M. (1996), L'enseignement en milieu scolaire, Paris, PUF.

Fodor J. (1986), La modularité de l'esprit, Paris, Minuit.

Mialaret G. (1991), Statistiques appliquées aux sciences humaines, Paris, PUF.

Pinker S. (1999), Comment fonctionne l'esprit, Paris, Odile Jacob.

PIот T. (2008), chapitre à paraître dans un ouvrage collectif du réseau OPEN sur l'observation des pratiques enseignantes, dirigé par M. Altet, C. Blanchard-Laville et M. Bru.

Postic M. (1977), Observation et formation des enseignants, Paris, PUF.

Reuter Y. (éd.) (2007), Dictionnaire des concepts fondamentaux des didactiques, Bruxelles, De Boeck.

Vergnaud G., Pastré P., Mayen P. (2006), «La didactique professionnelle (note de synthèse) ", Revue française de pédagogie, $\mathrm{n}^{\circ}$ 154, janvier-février-mars, p. 145-198.

WALbERG H.J. (1986), "Syntheses of research on teaching», in Handbook of research on teaching, M.C. Wittrock (éd.), New York, Macmillan.

11. Reuter 2007 . 



\title{
DE LA PSYCHOPÉDAGOGIE À L'ANALYSE PLURIELLE DES PRATIQUES
}

\begin{abstract}
Résumé: Historiquement, les sciences de l'éducation se sont constituées sur un fond ancien reposant, du côté de l'Université, sur le projet d'une science de l'éducation et la conception durkheimienne de la pédagogie comme théorie pratique et, institutionnellement, dans les Écoles normales, sur l'élaboration d'une discipline composite, la psychopédagogie, dont l'enseignement était confié à des professeurs de philosophie. Certes, les sciences de l'éducation ont trouvé leur appareillage conceptuel et leurs méthodes dans les disciplines mères, mais les caractéristiques de certaines de leurs questions (par exemple, l'analyse des pratiques enseignantes) démontrent aujourd'hui leur irréductible spécificité. De nouveaux paradigmes se sont progressivement imposés, se sont complexifiés, pour donner lieu à des recherches et à des théorisations originales. Par exemple, l'analyse plurielle des processus interactifs dans les situations d'enseignement et d'apprentissage, et la modélisation des pratiques enseignantes en termes d'équilibre et d'ajustement - en particulier à travers les travaux du réseau OPEN (Observation des pratiques enseignantes).
\end{abstract}

Mots clés: interaction, processus, médiation, pratique enseignante, analyse plurielle.

\section{Un rappel historique de l'émergence des sciences de l'éducation}

Dans ce colloque, qui fait le point sur les quarante ans de la discipline universitaire «sciences de l'éducation» telle qu'elle fut créée institutionnellement en France en 1967, notre propos sera de montrer son évolution à partir de l'importante question des rapports complexes entre la pédagogie, la psychopédagogie et la, les sciences de l'éducation, le domaine de l'éducation ayant pour spécificité de recouvrir à la fois un champ de pratiques et un champ de savoirs. Nous rendrons compte de l'évolution de notre discipline sous sa forme scientifique et plurielle telle qu'elle existe aujourd'hui, à partir de l'examen des travaux de recherche sur les pratiques pédagogiques, sur les pratiques enseignantes.

Des historiens ont dégagé l'origine à la fois philosophique et pédagogique de notre discipline et rappelé qu'il y eut bien avant, dès le début du XIX ${ }^{\mathrm{e}}$ siècle, des tentatives de pédagogie scientifique ou de science de l'éducation. Ce fut d'abord le projet de science positive de Marc-Antoine Jullien de Paris, en 1812, qui tenta de soumettre les pratiques éducatives à une rationalité scientifique et technique en créant une taxonomie "afin que l'éducation devienne une science à peu près positive». Puis fut mis 
en place, comme l'a montré J. Gautherin ${ }^{1}$, dans le souci politique d'édifier la Nation, de légitimer l'œuvre scolaire de la III ${ }^{\mathrm{e}}$ République et de former ses maîtres, un cours de science de l'éducation, institué par le ministère de l'Instruction publique dès 1883 . Ce cours fut assuré successivement par Buisson, Marion puis Durkheim et, au tournant du siècle, quinze des dix-sept facultés françaises disposaient d'un enseignement de science de l'éducation. Mais cette institutionnalisation, d'après Gautherin, ne s'explique ni par une volonté d'édifier la science au sens actuel de recherche scientifique susceptible d'éclairer les faits éducatifs, ni par une volonté d'outiller conceptuellement les pratiques pédagogiques, elle vient seulement de la volonté politique d'élever le niveau de formation des maîtres; les cours sont assurés par des professeurs de philosophie, spiritualistes pour la plupart, tous républicains, la science de l'éducation du XIX ${ }^{\mathrm{e}}$ siècle fut souvent, comme le décrit G. Compayré en 1879, un enseignement général, spéculatif et formel sur les fondements et les fins de l'éducation, de la morale et de la société. En France, la science de l'éducation fut donc à ses débuts une science spéculative.

$\mathrm{Au} \mathrm{XX}{ }^{\mathrm{e}}$ siècle, après une éclipse due à la guerre, la création, en 1967, des sciences de l'éducation au pluriel, comme discipline universitaire, par Gaston Mialaret, Jacques Wittwer, Jean Chateau, Maurice Debesse, répond aussi à une demande politique du ministère de l'Éducation, en novembre 1966, de créer un cursus en pédagogie au sein des universités françaises. Mais la mise en place d'une licence puis d'une maîtrise, en 1968, se situe dans le courant de développement des sciences humaines et sociales et reflète une demande de rapprochement entre la pédagogie et une approche par les sciences humaines de l'éducation, en particulier avec les apports de la psychologie. Cette évolution s'inscrit alors dans un nouveau paradigme scientifique.

\section{Les rapports complexes entre pédagogie et sciences de l'éducation}

Au moment de l'institutionnalisation de la discipline universitaire «les sciences de l'éducation », la pédagogie était encore l'affaire des «Écoles normales » et des professeurs de philosophie qui formaient les enseignants de l'enseignement primaire, avec l'appui d'enseignants de classes d'application, et se limitaient à un ensemble de recettes pour «bien faire la classe», reprochant, dit M. Develay, «à la pédagogie non pas d'être normative, mais de se poser comme la science directrice usurpant le rôle de la philosophie ${ }^{2}$. Quelques universités, pourtant, assuraient déjà un autre enseignement qui était, pour les uns, de la pédagogie générale, pour les autres, de la psychopédagogie ou de la philosophie et de l'histoire de l'éducation. L'enseignement donné dépassait ainsi déjà largement le «comment faire» pour aborder, soit sous l'angle philosophique, soit sous l'angle historique, soit sous l'angle scientifique en s'appuyant sur les apports des sciences humaines et sociales, les problèmes éducatifs.

1. Gautherin 2002.

2. Develay 2001, 14 . 
Différentes définitions sont données à la pédagogie entre pratique normative et théorie. Dans une nouvelle approche, Durkheim opère « une césure entre la pédagogie conçue comme une théorie-pratique et la science de l'éducation, science humaine plus encline à décrire et à comprendre qu'à suggérer des pratiques " ${ }^{3}$. Il tente de rendre compte des tensions entre les deux disciplines, en distinguant différents niveaux de sens du terme "pédagogie»:

- la pédagogie comme action et art de l'éducateur, du «bon pédagogue»;

- la pédagogie comme réflexion sur l'action éducative;

- la pédagogie comme systématisation de cette réflexion en une doctrine (la pédagogie Freinet, Montessori...).

La pédagogie comme théorie-pratique selon Durkheim est donc une théorie non scientifique qui vise à améliorer l'action, une discipline normative, un projet d'action construit en vue d'améliorer celle-ci. Alors que les visées de «la " science de l'éducation sont de l'ordre de la compréhension ou de l'explication des phénomènes éducatifs, la pédagogie cherche à juger ou à transformer l'action éducative: c'est une discipline praxéologique.

La pédagogie est donc une théorie mais ne se confond pas avec la science de l'éducation, qui est, écrit Durkheim, " tout entière à constituer », affirmant que l'éducation est susceptible d'une approche scientifique. Son objet, c'est l'ensemble des pratiques mises en œuvre par une génération sur la génération suivante, en vue de son adaptation au milieu social. Une science de l'éducation (l'histoire comparée, la sociologie des institutions) vise donc la connaissance des faits éducatifs sans préoccupation d'applications pratiques immédiates. Science de l'éducation et pédagogie ont bien le même objet mais pas le même intérêt théorique: les sciences de l'éducation visent la connaissance des faits éducatifs, la pédagogie recherche leur amélioration.

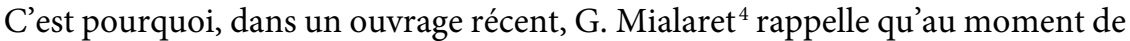
la fondation "des sciences de l'éducation» comme discipline universitaire, les universitaires réunis par le Ministère en novembre 1966 se mirent d'accord sur le titre de cette licence et de cette maîtrise qu'ils voulaient scientifiques et refusèrent d'emblée le terme, proposé par le Ministère, de licence de pédagogie, pour adopter celui de "licence de sciences de l'éducation" en s'inspirant de l'exemple de Genève, pour éviter «le mot de pédagogie devenu doublement équivoque». Ils ont souhaité ainsi marquer la rupture entre ce qui aurait pu être considéré comme un enseignement uniquement tourné vers une pratique scolaire et un enseignement basé sur l'ensemble des réflexions et des recherches scientifiques qui pouvaient définir un champ universitaire: celui des sciences de l'éducation.

Les sciences de l'éducation se situent dès lors dans un projet de connaissance et se construisent à partir de disciplines scientifiques descriptives et explicatives pour étudier «les conditions d'existence de fonctionnement et d'évolution des situations

3. Develay 2001, 18.

4. Mialaret 2006. 
et des faits d'éducation ${ }^{5}$, à partir de disciplines mères: psychologie(s), mais aussi, sociologie(s) (Isambert-Jamati), histoire de l'éducation (Beillerot), économie (Eicher), philosophie (Reboul, Best)... Progressivement, depuis quarante ans, elles se sont constitué un territoire propre autour d'objets complexes contextualisés, qu'elles traitent par des approches plurielles avec la pluridisciplinarité, l'inter-, la trans-, la codisciplinarité nécessaires.

Cependant, les fondateurs de la discipline, ceux de la "première génération", Maurice Debesse, Jean Chateau et Gaston Mialaret, avaient d'abord pensé créer une licence de «pédagogie» en 1962, puis une licence de "psychopédagogie». L'important traité que Maurice Debesse et Gaston Mialaret ont publié aux Presses universitaires de France, après la création des sciences de l'éducation entre 1969 et 1978, s'appelait Traité des sciences pédagogiques. On peut dire que les fondateurs institutionnels de la discipline n'avaient pas vraiment fait le deuil de l'idée d'une «science de l'éducation » qu'ils concevaient comme une science pédagogique ou bien comme une psychopédagogie scientifique.

\section{La psychopédagogie, une discipline de transition vers la scientificité}

La dénomination psychopédagogie ou psychologie pédagogique apparut avec Édouard Claparède, médecin, psychologue et éducateur, à l'occasion d'un séminaire donné à des institutrices: cette nouvelle discipline se voulait scientifique. Le terme correspond à « une pédagogie qui prend en considération des facteurs psychologiques dans l'acte éducatif»; c'est ainsi que Claparède la définit dans Psychologie de l'enfant et pédagogie expérimentale. Son objectif consiste au terme de ses recherches psychologiques à "aboutir à des conclusions pratiques pour l'action pédagogique».

Au même moment se développe le courant de la "pédagogie expérimentale», de la recherche expérimentale en pédagogie avec A.-A. Cournot, A. Binet, V. Henri, T. Simon, R. Buyse, R. Dottrens, A. Bonboir, G. de Landsheere, avec Gaston Mialaret enfin, qui insiste sur la nécessité de «faire profiter la pédagogie des progrès récents de la psychologie expérimentale en proposant les principes de l'expérimentation en pédagogie ». Il s'agit, écrit G. Mialaret, «de faire passer la pédagogie à l'ère de l'objectivité et de la preuve scientifique», de créer une pédagogie nouvelle fondée sur l'observation et sur l'expérimentation, sur une «recherche scientifique». G. Mialaret la définit comme "une pédagogie qui tient compte des processus psychologiques de l'élève et qui trouve son fondement scientifique dans la psychologie de l'éducation » et H. Piéron, psychologue, comme « une pédagogie scientifiquement fondée sur la psychologie de l'enfant». Ces auteurs posent comme principe le recours pour les pédagogues aux connaissances psychologiques de l'enfant, et l'essor des travaux d'Henri Wallon et de Jean Piaget va faciliter la rencontre de la pédagogie et de la psychologie. L'un et l'autre ont à la fois affirmé la distinction entre la pédagogie et la psychologie

5. Mialaret 1976. 
tout en montrant l'intérêt de développer des connaissances en psychologie pour permettre aux pédagogues de fonder leur action sur une pédagogie scientifique.

La psychopédagogie selon Gaston Mialaret, assistant de psychopédagogie à l'ENS de Saint-Cloud en 1946, directeur du premier laboratoire de psychopédagogie, puis, à Caen, fondateur du laboratoire de psychopédagogie en 1957 (étude des apports de la psychologie de Piaget et de Wallon), désigne à la fois « une théorie, une méthode et un ensemble de pratiques pédagogiques qui se réfèrent aux données de la psychologie de l'éducation », à la fois projet de connaissance et d'action ou «étude des composants psychologiques de l'action éducative».

Ainsi les travaux des années 1950-1970 de Mialaret ont porté sur l'analyse psychopédagogique de la communication en classe, de l'évaluation, des «méthodes faite sous l'angle psychologique», des problèmes d'apprentissage. Mialaret utilise aussi la notion d' "attitude psychopédagogique», comme prise de conscience des facteurs d'ordre psychologique en éducation.

De plus, institutionnellement, pour articuler pédagogie et apports de la psychologie, des chaires de «psychopédagogie» ont été créées dans les Écoles normales de formation d'instituteurs. Les professeurs de philosophie nommés psychopédagogues ou "professeurs de psychopédagogie» enseignaient à la fois la psychologie de l'enfant et la pédagogie générale. Dans les années 1970, ce sont ces philosophes qui s'attachèrent à réaffirmer dans la formation la distinction entre pédagogie et psychologie, soit en réintroduisant la philosophie de l'éducation comme fondement de l'éducation, soit en ouvrant les apports des sciences humaines et sociales à la pédagogie, psychologie certes, mais aussi sociologie, histoire..., et à s'inscrire dans le mouvement fondateur des sciences de l'éducation. Ici même au CERSE de Caen, dans les années 1980, Michel Fabre, Henri Peyronie et moi-même étions trois philosophespsychopédagogues en poste en École normale qui avons refait un cursus en sciences de l'éducation en privilégiant une entrée des sciences humaines dans nos travaux de recherches: H. Peyronie, la sociologie de l'éducation, $M$. Altet, la psychologie de l'éducation, M. Fabre, l'épistémologie et la psychanalyse de la connaissance, tout en illustrant le pluriel de notre nouvelle discipline. Comme l'écrit Francine Best en 2005, constatant un abandon progressif du terme composite de psychopédagogie: «sous l'effet des critiques qui lui ont été portées, tant du côté de la philosophie que de certaines sciences sociales, la psychopédagogie a été amenée à céder la place aux sciences de l'éducation, lesquelles bénéficient d'un statut de discipline universitaire».

La psychopédagogie avait bien joué le rôle de discipline transitoire avant le développement des sciences de l'éducation au pluriel.

\section{La création d'un nouveau champ scientifique: les sciences de l'éducation}

Avec la création et le développement des départements de sciences de l'éducation à Caen en 1967 par G. Mialaret, à Bordeaux, à Paris V, à Lyon, à Paris X, Paris VIII, Toulouse, et, en 2007, une trentaine de départements universitaires, la discipline 
s'institutionnalise; les départements s'adossent à des équipes de recherche et un nouveau champ scientifique se constitue sur le plan épistémologique: les sciences de l'éducation; ce sont des sciences plurielles descriptives et explicatives pensées à partir de sciences humaines et sociales «mères» qui vont se développer comme en atteste en 1973 le premier congrès «Apports des sciences fondamentales aux sciences de l'éducation ». À partir des travaux de G. Mialaret, la génération universitaire qui a suivi, les responsables universitaires de la discipline (ceux de la deuxième génération) ont conçu et développé les sciences de l'éducation à partir des sciences humaines et sociales «mères»: on est psychologue, sociologue, historien, anthropologue dans la discipline universitaire des sciences de l'éducation.

Ces dernières décennies ont montré, d'une part, qu'il existe des objets qui nécessitent la spécificité de l'approche plurielle, pluridisciplinaire, propre aux sciences de l'éducation, que, d'autre part, on relève des noyaux de recherche en éducation irréductibles aux recherches des autres sciences humaines, comme les pratiques enseignantes, l'évaluation, les didactiques disciplinaires, et qu'enfin il y a une façon de mener des travaux de sociologie ou de psychologie au sein des sciences de l'éducation qui se caractérise par une ouverture aux apports des autres sciences humaines dans le champ de la recherche, mais aussi par la construction d'objets spécifiques, sans oublier de s'intéresser aux formes de praxis éducative.

Dès 1994, G. Vigarello faisait le constat de la pluralité de ces sciences:

Les faits ici s'imposent et ils sont têtus: impossible, pour l'instant, d'y reconnaître quelque méthode ou quelque concept spécifique, impossible de définir une unité épistémologique. Il est inutile de refaire l'histoire des disciplines de sciences humaines pour montrer à quel point sont rares, sinon exceptionnels, les concepts transdisciplinaires. La quête mythique «d'une» science de l'éducation s'est effacée devant la réalité du multiple: la diversité est aujourd'hui reconnue, soulignée, travaillée.

À titre d'exemple, nous vous présenterons l'évolution en quarante ans des travaux sur les pratiques enseignantes.

\section{Les pratiques pédagogiques des enseignants: l'évolution d'un objet de recherche spécifique}

Dans les années 1970-1980, on assiste au développement des recherches en éducation sur les pratiques éducatives. Le champ de recherches appelé «la recherche éducationnelle» (Educational research) aux USA, au Québec comporte plus particulièrement la recherche sur les pratiques pédagogiques, sur l'enseignement, Research on teaching.

Ces travaux sont l'illustration d'un point de vue qui consiste à considérer la pédagogie comme objet de recherche à part entière et à identifier ce que les recherches en sciences de l'éducation ont apporté sur la description et la compréhension des processus à l'œuvre dans les pratiques pédagogiques. Les enseignants témoignent beaucoup de leurs activités en classe et de leurs difficultés, mais celles-ci avaient été peu étudiées 
objectivement à partir d'une analyse du fonctionnement des pratiques effectives. Des chercheurs de sciences de l'éducation ont pris pour objet les pratiques enseignantes, qui sont un de ces objets de recherche complexes, un objet carrefour spécifique aux sciences de l'éducation, qui relève d'une pluralité d'approches disciplinaires: psychologique, sociologique, pédagogique, didactique, épistémologique.

Les modélisations des travaux sur l'enseignement ont évolué ces cinquante dernières années. Historiquement, en Amérique du Nord, les premiers travaux sur l'enseignement dans les années cinquante se sont inscrits dans un paradigme behavioriste «processus-produit» à visée normative forte. Ils réduisaient l'étude de l'enseignement aux seuls comportements observables de l'enseignant. Ces travaux visaient à déterminer l'efficacité de l'enseignement, et les manières d'enseigner étaient analysées à partir des qualités personnelles intrinsèques de l'enseignant censées produire des effets sur les performances scolaires des élèves.

Les recherches qui ont suivi, relevant d'un paradigme cognitiviste (Clark, Peterson, Tochon) portaient sur «la pensée des enseignants». Elles concevaient la cognition en tant qu'instance essentielle de contrôle de la pratique enseignante, l'enseignant étant vu comme un décideur qui, à partir de ses pensées, théories et choix personnels, planifie ses actions et les met en œuvre. Puis l'émergence du paradigme «écologique» a permis de prendre en compte l'importance de la «situation ${ }^{6}$ au sein de laquelle se déroule l'enseignement (Doyle, Kounin), la situation devenant la variable clé explicative de la pratique.

Et ces quinze dernières années se sont développés les modèles interactionnistes: en France comme au Québec, des chercheurs (Altet, Bru, Clanet, Gauthier, Lenoir, Tupin, Vinatier) proposent des modèles intégrateurs qui visent l'articulation de plusieurs types de variables personnelles, processuelles et contextuelles en interaction. Les variables étudiées concernent l'enseignant, mais portent aussi sur l'élève et la situation, afin de pouvoir expliquer et comprendre le fonctionnement de la pratique enseignante dans sa complexité, à partir de l'étude des processus en jeu, de leurs interactions et des différentes dynamiques internes et externes.

Actuellement les travaux sur l'enseignement-apprentissage portent sur l'étude des processus caractéristiques des pratiques dans leur rapport avec les apprentissages des élèves ${ }^{7}$. Ces travaux montrent «l'impossible approche des pratiques enseignantes par les méthodes». Avant le développement de l'étude scientifique des processus en jeu, l'enseignement a été longtemps appréhendé par une approche prescriptive des «méthodes pédagogiques», de typologies de méthodes fondées sur des oppositions utilisées par les enseignants pour rendre compte de leurs pratiques: méthodes directives / non directives, traditionnelles / innovantes, expositives / interactives; cette vision réduisait la pratique d'un enseignant à la mise en œuvre méthodique d'une procédure préalablement établie.

\footnotetext{
6. Bronfenbrenner 1986.

7. Bruet al. 2004 .
} 
Les chercheurs travaillant aujourd'hui sur les processus ont des approches plus fines car, pour eux, la pratique ne se résume pas à une mise en œuvre qui serait toujours méthodique. Une pratique professionnelle recouvre des procédures, des produits mais aussi des processus interactifs, cognitifs, relationnels, psychologiques, contextuels. Le fait d'analyser les processus en rendant compte des dynamiques en jeu dans la pratique enseignante éloigne les chercheurs des modélisations de type entrée-sortie ou méthode. La pratique ne peut être considérée comme l'application d'une méthode, car chaque enseignant adapte à sa manière les caractéristiques de la méthode choisie. Une comparaison des pratiques observées de plusieurs enseignants censés mettre en œuvre la même méthode fait apparaître des écarts interindividuels importants et une grande variabilité. Le même enseignant met aussi en œuvre des procédures qui peuvent varier dans le temps en fonction des circonstances et des situations. Dans une recherche conduite sur deux années scolaires avec les mêmes enseignants du primaire, M. Altet, M. Bru, P. Bressoux et C. Leconte-Lambert ${ }^{8}$ établissent même que la variabilité intramaître, à conditions de travail différentes, est plus élevée que la variabilité intermaîtres à conditions égales. De même, plusieurs chercheurs montrent la difficulté à repérer une organisation méthodique de la pratique car, dans les faits, un enseignant ne règle pas méthodiquement son action en toutes circonstances mais est un bricoleur en situation. Enfin, si la pratique enseignante revient à réaliser des activités finalisées, ces finalités sont multiples et la notion de méthode, qui renvoie à la mise en œuvre de moyens organisés, tend à ignorer le caractère multifinalisé de la pratique enseignante. C'est le constat des limites de la notion de «méthode» qui a amené les chercheurs à construire de nouveaux modèles d'intelligibilité de la pratique enseignante et de son fonctionnement.

À côté de la recherche éducationnelle sur l'enseignement s'est fortement développée en France la recherche en didactiques des disciplines.

La recherche éducationnelle et les recherches didactiques ont un même objet de recherche, les processus en jeu dans «le triangle pédagogique» ou «le triangle didactique», les pratiques et interactions enseignants-élèves-savoirs, mais les travaux sont abordés avec des problématiques différentes et utilisent des concepts différents:

- la recherche éducationnelle traite l'objet du point de vue privilégié de l'enseignant, de ses actions, de ses interactions, des régulations, des ajustements avec l'élève en contexte (M. Altet);

- la recherche des didactiques disciplinaires l'aborde «du point de vue privilégié du contenu» (J.-L. Martinand), de sa structuration par l'élève en situation.

Les apports des recherches en didactiques disciplinaires sont importants au niveau des concepts qui permettent un éclairage des savoirs disciplinaires visés pour comprendre le fonctionnement des élèves confrontés à ces savoirs ainsi qu'une conceptualisation du contrat didactique, de la transposition didactique (Chevallard), du milieu et du temps didactique (Brousseau). L'analyse des situations didactiques du travail en

8. Altet et al. 1994-1996. 
classe a permis de montrer et de comprendre comment la question des apprentissages est contrainte par la forme scolaire et par la discipline enseignée.

Ainsi les recherches sur l'enseignement et les recherches didactiques sont deux territoires complémentaires à articuler, c'est ce que nous avons tenté de faire au CREN (Centre de recherche en éducation de Nantes) et au sein du réseau OPEN en faisant travailler ensemble sur un même corpus des chercheurs sur l'enseignement et des didacticiens. Notre recherche sur les pratiques enseignantes menée au CREN est l'illustration d'une recherche menée de façon pluridisciplinaire en sciences de l'éducation en France.

\section{Les recherches sur les pratiques enseignantes au CREN}

Nous avons d'abord mené des travaux descriptifs basés sur «l'observation » des «pratiques enseignantes» effectives en classe. Notre objectif était de décrire, caractériser et expliquer le fonctionnement des pratiques enseignantes, les processus en jeu à partir d'un modèle dit «des processus interactifs contextualisés » pour «comprendre les processus interactifs en situation ou l'articulation fonctionnelle des processus d'enseignement-apprentissage en situation ${ }^{9}$.

Nos premiers travaux ont étudié la pratique enseignante en classe à partir de son objet constitutif: l'interactivité, les interactions «pédagogiques, didactiques et intersubjectives» de l'enseignant, les ajustements et les décalages mis en œuvre dans le fonctionnement de la pratique enseignante. Ceci nous a fait mieux comprendre le processus d'adaptation interpersonnelle permanent en classe. Nous avions identifié des modes d'ajustement ou de non-ajustement entre enseignant et élèves en situation, par rapport à la préparation. Nous avions cherché à montrer que l'interactivité était un des organisateurs de la pratique enseignante et la recherche menée pour la DEP (Direction de l'évaluation et de la prospective) sur les pratiques des enseignants de $\mathrm{CE}_{2}{ }^{10}$ avait précisément dégagé une stabilité des interactions chez le même enseignant observé dans des classes différentes.

Nous avions essayé de caractériser les processus en jeu:

- au niveau des processus interactifs observables;

- au niveau des processus médiateurs sous-jacents: cognitifs, socio-affectifs, culturels;

- au niveau des processus situationnels.

Dans ces travaux, nous avions caractérisé la pratique enseignante à partir de processus, de variables d'actions définies par le chercheur, repéré des organisateurs stables des pratiques reflétant une organisation interne, comme le type d'interactions, de guidage, mais sans prendre en compte la gestion des contenus enseignés dans leur spécificité, ni les logiques d'action mises en œuvre par les différents acteurs; c'est pour réarticuler ces dimensions que nous avons développé au CREN l'analyse plurielle avec des approches conjointes de didacticiens et de psychologues.

\footnotetext{
9. Altet 1994.

10. Altet et al. 1994-1996.
} 


\section{Des approches complémentaires pour comprendre les processus en jeu: l'analyse plurielle}

Elle est menée par des chercheurs de sciences de l'éducation de disciplines différentes (M. Altet, M. Fabre, C. Morin, C. Orange, D. Orange, M. Hersant, M. Perraudeau, I. Vinatier...). L'analyse plurielle ${ }^{11}$ est une approche pluridisciplinaire descriptive et compréhensive des processus interactifs enseignement-apprentissage en situation et des processus d'apprentissage des élèves. La pluralité est motivée par la logique interne des recherches propres aux pratiques contextualisées; l'approche croisée des travaux de disciplines différentes sur les processus caractéristiques des pratiques et sur l'intentionnalité des acteurs, le sens de leurs actions permet de rendre intelligibles les pratiques enseignantes telles qu'elles existent dans leur diversité et de comprendre leurs relations avec les apprentissages des élèves dans des contextes variés. Les approches complémentaires «transversales», pour rendre compte de la complexité des processus et de la nature interactive et signifiante des pratiques en classe, se veulent plus heuristiques qu'une simple juxtaposition d'approches disciplinaires.

L'analyse plurielle est une question de partage entre chercheurs qui travaillent en commun sur le même corpus, cernent les variables en jeu, le poids de chacune selon leur entrée théorique, mettent en commun et confrontent leurs analyses, dégagent des invariants, identifient des différences, croisent leurs articulations pour dépasser les juxtapositions; ils tentent de construire ensemble une nouvelle lecture qui produise une plus-value dans l'intelligibilité de la pratique analysée et de produire des interprétations partagées. Ce cadre d'analyse produit de nouvelles grilles de lecture théoriques de la complexité des pratiques enseignantes observées, en analysant une activité enseignante singulière pour rendre compte de l'articulation des multiples dimensions et tensions en jeu; la démarche est fondée sur la confrontation de cadres théoriques différents (didactique, sociologique, clinique, analyse des interactions enseignant / apprenant, interactions langagières intersubjectives) pour comprendre les processus interactifs en jeu. Elle montre qu'il n'y a pas de métalangage donné a priori par une des disciplines, mais qu'un métalangage se construit à partir de la confrontation pluridisciplinaire avec ses propres concepts fédérateurs comme l'analyse des tensions et des régulations au cœur de la pratique (Altet) ou l'analyse de la co-activité (Vinatier). Les multiples dimensions, épistémique, pédagogique, didactique, psychologique et sociale, qui composent la pratique, interagissent entre elles au cours de l'adaptation de l'enseignant à la situation professionnelle et organisent la gestion conjointe de l'apprentissage des élèves et de la conduite de la classe.

C'est l'articulation entre ces différentes dimensions que l'approche plurielle des pratiques enseignantes s'efforce de mettre au jour. Pour ce faire, les chercheurs privilégient l'observation et la description de pratiques effectives, puis l'entretien avec l'enseignant observé, pour donner le sens de la pratique. Ce choix d'une approche

11. Altet 2002. 
multidimensionnelle de la pratique enseignante amène à reconsidérer une distinction entre, d'un côté, les travaux de didactiques disciplinaires, centrés sur la gestion des contenus, leur structuration et leur acquisition par les élèves, d'un autre côté, les recherches sur l'enseignement, la pédagogie, qui se focalisent sur la dimension communicationnelle de l'enseignement, et enfin les travaux psychologiques et sociologiques centrés sur les acteurs et leurs stratégies en classe. Ces travaux, longtemps présentés comme antagonistes et conduits séparément, apparaissent comme complémentaires si l'on veut rendre compte de la complexité de la pratique enseignante et de l'enchâssement dans l'action du didactique et du pédagogique.

Les chercheurs du CREN ont ajouté également à ce cadre d'analyse les significations données par l'enseignant à ses actions, ce qui a nécessité, dans nos recherches, de prendre en compte, à côté des observations, les interprétations des acteurs, en menant aussi des entretiens avec les enseignants sur leurs actions pour en saisir le sens, avant et après l'action observée, particulièrement l'entretien de co-explicitation. Enfin, ce cadre théorique englobe la phase de planification qui correspond, en amont, à la préparation didactique de la séquence. Cette phase se caractérise surtout par la structuration des contenus et s'avère très importante car elle permet de réduire les contraintes lors de la séquence. C'est une phase qui à la fois cadre et guide l'action, mais aussi la contraint par son scénario préétabli.

L'analyse plurielle permet ainsi d'intégrer par une approche pluridisciplinaire les diverses dimensions constitutives de la pratique dans leur spécificité, à partir d'entrées de recherches ${ }^{12}$ disciplinaires différentes, et d'en comprendre l'articulation et le fonctionnement. Sur le plan méthodologique, trois types de recueils de données sont utilisés: des observations systématiques, des entretiens avec les acteurs et des préparations de l'enseignant ou des productions des élèves.

Tel est l'objet d'une analyse plurielle qui se propose de croiser les approches disciplinaires et de contribuer à les relier. Il reste à traiter la question de la contingence des types de chercheurs qui mènent les analyses croisées.

\section{Une modélisation de la pratique enseignante}

Ces trente ans de travaux nous amènent à proposer un modèle d'intelligibilité de la pratique enseignante. Dans un métier de l'humain, dans une pratique communicationnelle comme l'enseignement, où l'enseignant agit dans l'incertitude, où ses actions sont « relayées » ou non par les élèves, où les trames d'interactions structurent l'action à travers plusieurs dimensions de la pratique, les tensions à gérer sont nombreuses. Ce sont ces processus interactifs en tensions que l'analyse plurielle s'efforce de mettre au jour. À partir de l'observation de pratiques effectives, il s'agit de dégager les tensions organisatrices et d'identifier des processus interactifs stables, des organisateurs (type d'interactions, guidage, configuration de la tâche), de mettre en évidence la cohérence

12. Altet 1994, 2002. 
de la dimension communicationnelle de l'enseignement et son articulation avec le volet didactique, d'analyser les dynamiques interactives et leurs régulations, d'identifier l'interactivité fonctionnelle entre enseignant et apprenants; ce sont les trames interactionnelles avec les élèves qui commandent la logique du travail enseignant et produisent une co-activité ${ }^{13}$. L'analyse recouvre bien les dimensions pédagogiques, didactiques, intersubjectives dont il s'agit de comprendre l'articulation fonctionnelle, la cohérence et l'équilibre de fonctionnement. Le modèle d'analyse de la pratique est celui de processus en tensions à maîtriser par des régulations, des transactions, des ajustements pour gérer des logiques d'action contradictoires pédagogiques, didactiques, épistémiques, sociales, psychologiques, personnelles en jeu et obtenir un équilibre entre les gestions du savoir, de l'élève et de la dynamique de la situation ${ }^{14}$. Nous avons intégré une autre caractéristique des approches interactives: le fait qu'elles considèrent que le processus enseignement-apprentissage fonctionne comme un travail interactif d'ajustements, de négociations, de transactions et de compromis permanent entre les acteurs en situation. L'enseignement met en jeu des rapports entre enseignant et élèves où sont convoqués le langage, l'affectivité, la personnalité et qui comportent, comme dans toutes les relations humaines, des rapports de pouvoir, des résistances, des initiatives avec négociation, contrôle, séduction, persuasion. Le sens de l'action en classe est construit par les acteurs au travers de leurs négociations. Ce cadre interprétatif qui s'inscrit dans un modèle constructiviste et interactionniste permet ainsi d'articuler aux variables didactiques et pédagogiques les variables intersubjectives (psychologiques et sociologiques) en jeu dans le processus interactif, et considère que la pratique enseignante est toujours affaire d'ajustement.

Mais il n'y a pas une seule modélisation de la pratique enseignante, et les confrontations entre équipes de sciences de l'éducation permettent d'enrichir notre intelligibilité de cet objet.

\section{Les travaux du réseau OPEN (2001-2007)}

Les chercheurs de vingt-trois équipes françaises et internationales se rencontrent pour confronter leurs travaux autour des objectifs communs suivants:

- décrire, caractériser, comprendre le fonctionnement des pratiques enseignantes;

- répertorier les travaux existants;

- poursuivre la clarification des concepts et des notions utilisées dans différents paradigmes, par des approches disciplinaires différentes;

- mettre en perspective nos recherches françaises avec les productions internationales;

- rassembler et cumuler les données d'enquête sur une période assez longue;

- enfin, aboutir à la construction d'un outil destiné à rendre compte par observation des pratiques enseignantes telles qu'elles sont.

13. Vinatier \& Altet 2008.

14. Altet 2008. 
Le réseau est né des réflexions communes de trois équipes fondatrices: CREN (M. Altet), CREFI (M. Bru), CREF (C. Blanchard-Laville):

- nos travaux partent d'observations de pratiques effectives;

- nous n'utilisons pas la notion de «méthode d'enseignement» pour désigner les pratiques enseignantes;

- nous nous intéressons aux processus, aux procédures, aux produits;

- nous prenons en compte le contexte, la contextualisation de la pratique;

- nous n'expliquons pas la pratique en la ramenant soit à un acte délibéré de l'enseignement soit à des déterminismes extérieurs;

- nous considérons qu'il existe plusieurs manières de rendre raison des pratiques selon l'approche, selon l'échelle, selon le croisement des entrées choisies;

- pour nous «la pratique» est une notion englobante qui comprend les actions, les actes, l'activité, le travail de l'enseignant;

- nous menons des recherches pluri-, inter- ou co-disciplinaires sur les pratiques enseignantes pour en comprendre le fonctionnement.

Les chercheurs du réseau OPEN s'efforcent de mettre en œuvre une articulation des références théoriques utilisées, la mobilisation de plusieurs angles d'approches, de plusieurs méthodologies pour tenter de dépasser les dualismes.

Des approches pluri, inter, co-disciplinaires entre les équipes sont confrontées:

- approche plurielle des processus en tension et approche des organisateurs (CREN, CREFI);

- approche de plusieurs niveaux d'analyse micro, meso, macro (Tupin);

- approche double didactique et ergonomique (Robert, Rogalski);

- approche par la théorie de l'activité de Vergnaud et la théorie linguistique des interactions de Kerbrat-Orecchioni et l'activité productive / transformative (Vinatier);

- approche par la cognition située et l'opérativité Occhanine (Piot);

- approche du générique et du spécifique de l'action de l'enseignant (Sensevy, Amade-Escot);

- approche langagière et ergonomique (Bucheton);

- approche de la didactique professionnelle: articulation analyse du travail et conception de la formation (Pastré);

- approche de l'intervention éducative (Lenoir).

Nous avons ainsi, avec les chercheurs des différentes équipes, d'abord délimité, construit et redéfini l'objet: pratique enseignante, activité de l'enseignant, action de l'enseignant, travail enseignant, acte pédagogique, intervention pédagogique: chaque dénomination définit différemment l'objet. Nos recherches en sciences de l'éducation montrent que l'étude de l'objet «pratique enseignante» recouvre à la fois la pratique, l'agir pédagogique et l'agir didactique. Dans son travail, l'enseignant remplit simultanément une fonction pédagogique de médiation entre le savoir et l'élève, de gestion de la classe et une fonction didactique de gestion des apprentissages, dans un contexte singulier, contraint, dynamique. Étudier le volet «pratique pédagogique» de l'enseignant, c'est se centrer sur les actes de médiation mis en œuvre entre le savoir 
et l'élève sur la communication. Étudier la médiation, c'est analyser la médiation du pédagogue, de la personne: la relation, les interactions pédagogiques, les processus interactifs, cognitifs, affectifs de la communication verbale, non verbale, les processus intersubjectifs en jeu entre les acteurs, identifier les variables d'action. C'est aussi analyser la médiation des dispositifs pédagogiques, l'instrumentation mise en place, la situation choisie pour favoriser l'engagement dans la tâche, la transformation d'informations en savoirs chez l'élève.

Les travaux sur les pratiques enseignantes relient les approches sur les actions pédagogiques de l'enseignant en situation, en interaction avec les apprenants, situées dans le temps et les approches didactiques qui analysent les savoirs et les apprentissages en jeu dans la situation étudiée. Les modélisations utilisées, «modèle des processus interactifs situés ", "modèle clinique», "modèle des organisateurs et des processus en tensions», permettent de comprendre le sens de ces médiations pédagogiques liées à un contrat didactique. Les travaux de sciences de l'éducation associés aux approches de l'ergonomie, de l'anthropologie cognitive située, de la sociolinguistique, de la sociologie du travail permettent de comprendre le fonctionnement des pratiques pédagogiques en situation.

Ces travaux croisés ont produit des savoirs sur la multidimensionnalité des pratiques en classe, sur l'articulation des logiques d'action, sur l'analyse de l'interactivité en classe (Altet), sur les deux volets de l'activité enseignante, productive et transformatrice (Vinatier), sur l'analyse des organisateurs de la pratique (Bru), sur l'analyse de l'activité enseignante située (Piot), sur l'analyse des gestes professionnels (Amigues), sur l'analyse sociologique du travail enseignant (Tardif, Barrère), sur l'analyse du générique et du spécifique (Amade-Escot, Sensevy).

De plus, à côté de l'approche plurielle du CREN présentée ci-dessus, chaque équipe propose une théorisation qui problématise d'autres volets de la pratique enseignante.

Ainsi, M. Bru ${ }^{15}$ et le CREFI cherchent à dégager des organisateurs de la pratique enseignante (et non des déterminants). Une pratique est organisée, s'organise et est organisatrice. Les organisateurs des pratiques ne sont pas des facteurs, c'est en termes de processus organisateurs (représentations, schèmes, couplages action-situation, configuration de la classe, type de tâches, de guidage) qu'il importe de penser la notion d'organisateurs de la pratique. Dans le souci de mieux connaître la façon dont sont organisées et s'organisent les pratiques enseignantes, un intérêt particulier est accordé à leur contextualisation, celle interne des composantes de la pratique: la contextualisation temporelle (séquentielle) des configurations de la pratique et la contextualisation «externe» de la pratique d'enseignement.

Dans une autre approche, clinique et co-disciplinaire (psychologie clinique, didactique des mathématiques), plusieurs modèles théoriques contribuent à l'analyse d'une même pratique. Claudine Blanchard-Laville utilise la notion globale d'acte d'enseignement plutôt que d'action, au sens de G. Mendel. Pour l'approche clinique, il n'y

15. Bru 1991. 
a pas d'acte sans un projet d'action qui se continue durant le temps de l'acte. L'acte a une dimension effective, engagée dans le concret. La pratique est une notion plus englobante qui reprend l'ensemble des actes mais ne s'y résume pas. Elle inclut la posture, c'est-à-dire le rapport au savoir, à soi-même et aux autres.

Pour les didacticiens, l'objet d'étude est le fonctionnement du système didactique en tant que relation ternaire entre une instance enseignante, une instance élève(s) et un objet de savoir. La modélisation de l'action du professeur constitue l'objet de travail des didacticiens comparatistes et la distinction générique / spécifique l'un de leurs outils pour comprendre son activité. La question est abordée en termes de «milieu», le milieu de l'élève et le milieu du professeur. Le "milieu» a un rôle de médiation (medius / mesos) dans «l'action conjointe» du professeur et de l'élève.

Dans l'approche sociologique, les pratiques enseignantes sont analysées au regard des possibilités, «des marges d'autonomie», «des stratégies des enseignants » pour favoriser la démocratisation de l'accès aux savoirs; elles sont examinées en référence à la triade conceptuelle «efficacité-efficience-équité». Les travaux ont décrit méthodiquement et analysé les relations entre les dynamiques interactives en classe, les apprentissages et les progressions des élèves, en précisant quelles formes d'interactions offrent aux élèves les meilleures conditions d'apprentissage ${ }^{16}$.

Dans tous ces travaux, il ne s'agit pas d'expliquer la pratique professionnelle en la ramenant soit à un acte souverain et parfaitement délibéré de l'enseignant, soit au seul résultat de déterminismes extérieurs; il s'agit de montrer qu'il existe plusieurs façons de rendre raison des pratiques, et qu'un dialogue entre les équipes et leurs différentes façons de rendre raison des pratiques est possible et scientifiquement profitable, à partir du moment où ces équipes arrivent à partager des hypothèses communes de recherche sur l'indispensable connaissance des processus en jeu. Une question centrale demeure: toutes les pratiques professionnelles des enseignants ne se valent pas. Les recherches ont jusqu'à présent quantifié « un effet-maître " général, il reste à comprendre en quoi consiste cet effet-maître, quels en sont les processus constitutifs, en développant des travaux sur les effets des pratiques sur les apprentissages des élèves dans la diversité des situations d'enseignement-apprentissage.

Ces travaux de recherche en sciences de l'éducation sur la pédagogie se distinguent de la recherche-action qui cherche d'emblée à modifier les pratiques ou encore de la recherche pédagogique au sens de recherche sur les méthodes pédagogiques, sur les modèles d'enseignement, qui sont des modèles pour l'action, posent a priori des finalités pratiques à atteindre et qui produisent des outils pour l'action. Ils diffèrent aussi de travaux purement didactiques centrés sur la structuration des savoirs ou les processus d'acquisition des savoirs par l'apprenant et les complètent. Ces travaux permettent de dégager la nécessaire convergence entre le didactique et le pédagogique pour que le processus enseignement-apprentissage fonctionne. Ils visent à rendre intelligible le processus enseignement-apprentissage en identifiant

16. Génélot \& Tupin 2003. 
les différentes variables en interaction. Les travaux sur «l'effet-maître» cherchent à identifier les variables effectives de la pratique pédagogique. Ces recherches fournissent des repères théoriques, des grilles de lecture des pratiques, qui peuvent être transposés dans la formation des enseignants.

En conclusion, en quarante ans, les sciences de l'éducation sont devenues des sciences matures et légitimes sur le plan scientifique: elles se sont séparées des approches pédagogiques prescriptives; elles ont inventé un point de vue pluriel, pluri- ou interdisciplinaire sur des objets qui leur sont propres; elles travaillent sur des zones frontières spécifiques; elles ont construit des savoirs, des concepts propres; elles ont produit des théories réinventées; elles ont développé un corpus de connaissances validées, reconnues; elles ont rendu compte de processus éducatifs rendus intelligibles autrement; elles ont travaillé sur les rapports entre savoirs savants et pratiques. Ces avancées restent à poursuivre, il s'agit à présent de:

- continuer à cumuler les travaux;

- poursuivre l'articulation des références théoriques des différentes disciplines et construire des théorisations propres;

- faire des synthèses des acquis des recherches et développer les conférences de consensus pour diffuser les connaissances;

- articuler les connaissances scientifiques avec d'autres modes de connaissance communs et développer la philosophie de l'éducation comme questionnement critique, comme réflexivité sur les savoirs scientifiques.

En tant que chercheure mais aussi institutionnelle comme directrice d'un IUFM, il me semble que les sciences de l'éducation ont à l'heure actuelle une chance à saisir et une dérive à éviter : réussir l'articulation des recherches en sciences de l'éducation et la formation des enseignants avec l'intégration des IUFM à l'Université, tout en ne se laissant pas dériver vers une «neuropédagogie» annoncée par les neurosciences!

Marguerite Altet

CREN

Université de Nantes 


\section{Références bibliographiques}

Altet M. (1994), La formation professionnelle des enseignants, Paris, PUF.

Altet M. (2002), «L'analyse plurielle de la pratique enseignante, une démarche de recherche ", Revue française de pédagogie, $\mathrm{n}^{\circ} 138$.

Altet M. (2006), « Les enseignants et leurs pratiques professionnelles», in Traité de la formation, J. Beillerot, N. Mosconi (dir.), Paris, Dunod.

Altet M. (2008), L'analyse de pratiques: une démarche de formation et de recherche, Paris, PUF.

Altet M., Bressoux P., Bru M., Leconte-Lambert C. (1994-1996), Étude exploratoire des pratiques enseignantes de CE2, Paris, DEP.

Bronfenbrenner U. (1986), «Dix années de recherche sur l'écologie du développement humain", in L'art et la science de l'enseignement. Hommage à Gilbert de Landsheere, M. Crahay, D. Lafontaine (éd.), Bruxelles, Labor.

BRU M. (1991), Les variations didactiques dans l'organisation des conditions d'apprentissage, Toulouse, Éditions universitaires du Sud.

Bru M., Altet M., Blanchard-Laville C. (2004), « La recherche des processus caractéristiques des pratiques enseignantes dans leurs rapports aux apprentissages », Revue française de pédagogie, $\mathrm{n}^{\circ}$ 148, p. 75-87.

Develay M. (2001), Propos sur les sciences de l'éducation. Réflexions épistémologiques, Paris, ESF (Pratiques et enjeux pédagogiques).

GAUTHERIN J. (2002), Une discipline pour la République: la science de l'éducation en France (1882-1914), Berne, Peter Lang.

GÉNÉLOT S., TUPIN F. (2003), « Méthodologie de l'évaluation des pratiques enseignantes», in EVLANG. L'éveil aux langues à l'école primaire. Bilan d'une innovation européenne, M. Candelier (dir.), Bruxelles, De Boeck, chap. 4, p. 95-122.

Mialaret G. (1976-2005), Les sciences de l'éducation, Paris, PUF.

Mialaret G. (1984-1996), La pédagogie expérimentale, Paris, PUF.

Mialaret G. (1987-1998), La psychopédagogie, Paris, PUF (Que sais-je?).

Mialaret G. (2006), Sciences de l'éducation, Paris, PUF (Quadrige).

Vinatier I., Altet M. (dir.) (2008), Analyser et comprendre la pratique enseignante, Rennes, Presses universitaires de Rennes. 



\title{
DIDACTIQUE ET SCIENCES DE L'ÉDUCATION : UNE RECONFIGURATION?
}

\begin{abstract}
Résumé: Privilégier l'analyse des contenus et de leur transmission / acquisition conduisit les premières recherches en didactique à négliger la dimension épistémologique des pratiques enseignantes, mais l'option anthropologique, dans sa généralité même, ne va pas sans difficulté. G. Sensevy suggère que les recherches françaises auraient tout à gagner des recherches nord-américaines sur l'analyse des savoirs pédagogiques en eux-mêmes, et constate que la pluridisciplinarité des recherches, en France, plaide en ce sens. Il resterait, à ses yeux, à lier davantage les recherches positives portant sur les faits à la réflexion sur les valeurs.
\end{abstract}

Mots clés : épistémologie pratique, valeur, pluridisciplinarité, pédagogie.

\section{Introduction}

Ce texte constitue une sorte de perspective, brossée à grands traits. Il n'a pas vocation à faire de l'histoire, ni à produire des concepts. Son objectif consiste avant tout dans un début de problématisation de la question de la reconfiguration des recherches en éducation, au premier rang desquelles celles accomplies en didactique et en sciences de l'éducation. Il s'agit donc d'une (petite) entreprise résolument tournée vers le futur, dans le sens où elle postule un tropisme dont les effets sont encore largement à venir, tropisme à la puissance duquel elle cherche à contribuer.

\section{La naissance des didactiques: la centration sur le contenu}

Dès les premiers moments, la didactique s'est rapidement définie comme la discipline qui étudiait ce qui est, dans les activités d'enseignement et d'apprentissage, spécifique des contenus.

Dans ces premiers moments, une certaine division du travail pouvait être respectée: aux chercheurs en sciences de l'éducation et aux pédagogues une étude générale ou générique des pratiques, aux didacticiens l'étude spécifique de l'enseignement et de l'apprentissage dans telle ou telle discipline, du point de vue de tel ou tel contenu.

Cette division du travail ne signifiait pas nécessairement une harmonie heureuse entre tenants de l'une ou l'autre approche. Dans une certaine mesure, la didactique 
pouvait se constituer dans la critique des sciences de l'éducation ou de la pédagogie " oublieuses des savoirs", tandis que ces dernières pouvaient reprocher à la nouvelle venue son "réductionnisme», voire son "positivisme».

Une chose déjà pouvait commencer de frapper, au-delà de cette division du travail, c'est le type de relation établi entre sciences de l'éducation "générales » et didactiques spécifiques: une relative ignorance réciproque s'installa vite, parsemée d'éclairs d'hostilité, et de quelques rares oasis de travail commun. Mais on peut raisonnablement conjecturer que les travaux des uns n'étaient pas connus par les autres, et réciproquement. Il faut noter que cette ignorance réciproque s'étend(ait) également aux rapports entre didactique, d'une part, et sociologie de l'éducation et psychologie de l'éducation, d'autre part, même si cette situation pouvait être assez différente selon les didactiques. Au-delà de cette forme de constat, on peut noter de façon incidente qu'il y aurait certainement un livre à écrire ou un colloque à organiser sur la manière dont une communauté de recherche peut subsister malgré (ou grâce à) de si fortes ignorances.

\section{L'aporie de la centration sur les contenus}

Une évolution, qui d'ailleurs actualisait une tendance déjà présente depuis le début dans les travaux de certains didacticiens, fut la suivante: la prise de conscience que la seule centration sur le contenu de savoir ne pouvait suffire à définir une discipline comme appartenant aux sciences de l'homme et de la société. Cette constatation fut en grande partie la conséquence d'études empiriques; on la décrira à peu près comme ceci: étudier ce qui est spécifique du contenu nécessite d'intégrer des aspects de l'enseignement et de l'apprentissage qui ne sont pas eux-mêmes directement spécifiques du contenu. Parmi ces aspects, certains, particulièrement importants, furent découverts de la manière suivante: mettant en œuvre des ingénieries didactiques produites par les didacticiens, les professeurs donnaient à voir d'autres rapports aux objets de savoir que ceux des didacticiens eux-mêmes. Ces rapports étaient autres en grande partie par ce que j'ai appelé «l'épistémologie pratique» des professeurs, c'est-à-dire la théorie de la connaissance qui était la leur (théorie à la fois générique et spécifiée à tel ou tel savoir), produite par la pratique et la produisant en retour, cette épistémologie pratique était relativement incompatible avec la mise en œuvre adéquate du dispositif pensé par les didacticiens.

Les didacticiens devaient donc aller jusqu'au bout, et abandonner le mythe de la transparence des savoirs. Enseigner, apprendre, sont des actions, des activités, des pratiques, qui ne sauraient se résumer dans la seule description plus ou moins savante des objets de savoir, dont des avatars plus ou moins reconnaissables constituent l'enjeu et la cible de l'action didactique conjointe du professeur et des élèves ${ }^{1}$.

1. Pour les premiers éléments d'exposition d'une théorie de l'action conjointe en didactique, voir Sensevy \& Mercier 2007 et Schubauer-Leoni et al. 2007. 
Une autre des difficultés rencontrées tenait à un fait plus général: la prise de conscience de déterminants de l'action autres que purement didactiques, et pourtant d'influence profonde. On pouvait reconnaître par exemple la surdétermination sociale de certains comportements d'élèves, ou de certains comportements professoraux. Même si la prise en compte directe de problématiques de type sociodidactique par les didacticiens (et par les sociologues ${ }^{2}$ ) fut (et reste) rare, il devenait de plus en plus difficile de s'en tenir à ce qui est «spécifique des contenus».

\section{Les difficultés d'une perspective actionnelle}

La didactique devait se confronter ainsi à un dilemme difficile à dépasser. Soit elle acceptait de se confiner à l'espace raréfié des études de ce qui est spécifique des savoirs dans l'enseignement et l'apprentissage, mais cela sous peine de renoncer à une compréhension réelle des phénomènes didactiques eux-mêmes, dès lors que l'on pouvait montrer que les phénomènes didactiques étaient dans certains cas en partie déterminés par autre chose qu'eux-mêmes.

Soit elle acceptait sa vocation anthropologique: un bon exemple d'un tel parti pris est donné par le travail d'Y. Chevallard, dont la dernière définition de la didactique est de ce point de vue éclairante. Il commence par construire une notion de praxéologie qui englobe l'activité humaine, comme un ensemble tâche technique et théorie. Ceci fait qu'indifféremment "résoudre une équation du second degré», "se couper les ongles des pieds», "composer une symphonie», "aborder une personne dans la rue pour nouer une amourette» ou «aller aux toilettes» sont des praxéologies.

Chevallard donne ensuite cette définition de la didactique:

Il faut tirer les conséquences de la rupture épistémologique - qui, comme toute rupture de cet ordre, est d'abord une rupture dans la culture - que le concept de praxéologie institue. Par cette rupture, la didactique peut être conçue comme la science - dont il n'existe aujourd'hui que des fragments - ayant pour objet d'étude les processus de diffusion des praxéologies dans la société. Ou plutôt, la didactique est la science des conditions, des contraintes, des mécanismes de la diffusion praxéologique. Son territoire est donc immense. Elle suppose, bien entendu, des spécialisations; mais celles-ci, lorsqu'elles ne se résignent pas à suivre servilement le découpage des institutions établies, ne vont nullement de soi ${ }^{3}$.

On peut donc imaginer que chacune des praxéologies évoquées ci-dessus pourrait sans peine être étudiée du point de vue de sa diffusion, ce qui suppose, on le voit, d'étudier nécessairement de la façon la plus fine son contenu, pour pouvoir attester de ses modifications, et des orientations que ce contenu fournit à l'expérience. Il faut prendre conscience de l'intérêt d'une telle définition, qu'on l'accepte, qu'on la modifie,

\footnotetext{
2. Lahire 2007 .

3. Chevallard 2007, 17 .
} 
ou qu'on la repousse en bloc, dans le fait qu'elle modifie radicalement la perspective précédente, et qu'elle confie à la didactique le statut d'une science de la pratique.

On voit d'entrée les difficultés soulevées par cette double perspective. D’un côté, celui de la didactique resserrée sur le spécifique, un risque « déflationniste » : la pertinence de la didactique est peut-être avérée, mais tellement restreinte qu'on peut douter de l'intérêt à y investir de l'énergie. Les critiques antiréductionnistes, par ailleurs, trouveront un terrain d'expression privilégié. On pressent que cette définition déflationniste, du "pré carré», n'a pas que des désavantages en termes de voisinage social, et certains pourront apprécier le cantonnement, qui assure à la fois la sauvegarde de ses propres territoires et la facilité de la critique d'autrui.

De l'autre, celui de la didactique à vocation anthropologique, un risque « inflationniste». Une lecture rapide de la définition de Chevallard pourrait faire poser la question faussement naïve suivante: "Mais alors, qu'est-ce qui n'est pas de la didactique?». À mettre en avant une ambition aussi démesurée, ne risque-t-on pas de trop embrasser pour mal étreindre? Même si l'on conçoit des spécialités, comment penser une didactique qui fasse droit à des déterminants cognitifs, sociologiques, historiques, etc.?

Ainsi pourrait aller (assez mal) la didactique: tour à tour vilipendée comme montagne un peu ridicule qui accouche d'une souris bien chétive, ou comme une cathédrale boursouflée jetant ridiculement son dévolu sur l'ensemble des sciences de l'homme et de la société dans un impérialisme malade.

Bien entendu, je vais montrer qu'on peut sortir d'un tel dilemme. Mais avant de tenter une réévaluation de l'état des lieux, je voudrais faire un détour anglo-saxon.

\section{Un exemple dans le monde anglo-saxon}

Dans le monde anglo-saxon, de didactique, au sens strict de ce terme, il n'y en a pas eu. Mais on pourrait faire état, cependant, d'un mouvement de même type, qu'on pourrait dater par exemple avec un texte profond, écrit en 1986, de Lee Shulman ${ }^{4}$.

Celui-ci s'attaque dans ce texte à la fameuse maxime de Shaw: He who can, does. He who cannot, teaches. Dans ce texte, Shulman produit plusieurs fortes assertions, dont une en particulier: comprendre le travail d'un professeur, comprendre la nature de ce travail, et donc la manière dont on doit former un professeur, c'est comprendre comment le professeur doit élaborer un rapport particulier aux contenus qu'il enseigne. Le professeur doit en effet non seulement maîtriser les contenus per se comme le ferait un diplômé de la discipline, mais il doit de plus identifier dans ce contenu la substance et la syntaxe qui lui permettront de faire comprendre à ses élèves ce contenu. Cela suppose aussi, nous dit Shulman, qu'il connaisse les conceptions possiblement problématiques des élèves sur un contenu donné et sache travailler avec elles. De fait, en 1985, alors que la didactique est naissante en France, Shulman, d'ailleurs, dans un parallèle constant et toujours fructueux professeur / médecin, invente une

4. Shulman 2007 . 
sorte de didactique à l'américaine, centrée sur les savoirs, définissant ainsi, à côté du content kwowledge qui ne saurait caractériser que par défaut le savoir professoral, le pedagogical content knowledge, que tout didacticien d'aujourd'hui pourrait nommer sans difficulté, me semble-t-il, savoir didactique, puisqu'on peut le définir comme la connaissance que l'on a d'un savoir à des fins d'enseignement.

Je m'arrêterai un instant sur le travail de Shulman en notant les aspects suivants:

- dans l'ensemble de son texte, et plus généralement dans l'ensemble de son travail, Shulman ne produit aucune exclusive et ne se fait pas contempteur. S'il regrette qu'un très grand nombre, pour ne pas dire la quasi-totalité des recherches entreprises sur l'enseignement et l'apprentissage, évacuent les contenus, il est loin de penser que seules les recherches centrées sur l'étude des diverses formes de pedagogical content knowledge devraient être menées. Au contraire, il plaide continûment, dans une perspective finement historique, pour l'assomption de la complexité de l'enseignement, et donc pour le voisinage raisonné de recherches d'inspiration très différente;

- l'influence du texte de Shulman est très grande aujourd'hui aux États-Unis et plus généralement dans le monde anglo-saxon. Les récentes livraisons de revues comme Cognition \& Instruction, le Journal for Research in Mathematics Education, ou le Journal of the Learning Sciences contiennent ainsi de forts articles articulés précisément autour de la notion de pedagogical content knowledge, citant chacun expressément le texte de Shulman. On notera par ailleurs qu'un livre comme celui de Liping $\mathrm{Ma}^{5}$, préfacé par Shulman, a joué un rôle très important au sein des communautés de recherche américaines en éducation dans la mise à distance du socioconstructivisme, et dans une centration réaffirmée sur les savoirs. Il n'est pas indifférent de savoir que le livre de Liping Ma compare le pedagogical content knowledge, en mathématiques, d'enseignants du premier degré américains et chinois, et que la différence semble écrasante en faveur des Chinois.

Il m'apparaît que ces avancées anglo-saxonnes peuvent nous aider, à la fois dans leur pragmatisme (le fait d'étudier les pratiques telles qu'elles se font et d'établir à partir de cette étude des coopérations avec les professeurs pour la définition de dispositifs spécifiques), et dans la manière dont elles intègrent comme sans y penser, au sein d'une même recherche, des considérations qu'on s'attendrait plutôt, dans les études francophones, à voir dispersées tantôt dans des recherches de sciences de l'éducation tantôt dans des recherches de didactique.

\section{Un essai d'état des lieux?}

Revenant dans le monde francophone, et plus particulièrement en France, je voudrais montrer que le dilemme déployé plus haut est plutôt abstrait qu'effectif.

5. Liping Ma 1999. 
Je serai tenté de répondre positivement à la question que nous essayons de traiter ensemble: "Quarante ans des sciences de l'éducation: l'âge de la maturité?».

Je pense en effet que les sciences de l'éducation sont à l'âge de la maturité, du moins à son abord. J'évaluerai en effet de la manière suivante l'état des lieux aujourd'hui.

(Certains) didacticiens et (certains) chercheurs en sciences de l'éducation travaillent aujourd'hui ensemble. Qu'on prenne l'exemple des réseaux internationaux OPEN et RESEIDA, et on verra que les problématiques deviennent partagées, les regards pluriels / croisés systématiques. Il semble qu'on trouve là de réelles occasions d'avancer, en particulier parce qu'il se pratique dans ces rencontres, pour parler comme Paul Veyne $^{6}$, une certaine forme d'allongement du questionnaire. Ont ainsi droit à l'expression et à l'enquête des questions d'origine didactique, qui, en se posant dans ce contexte, gagnent en profondeur et en pertinence: par exemple, on pourra se rendre attentif à la manière dont les savoirs donnent leurs formes aux interactions, qu'il apparaîtra désormais difficile, voire périlleux, d'étudier en apesanteur didactique ou épistémique. D’une manière symétrique, les didacticiens confrontés aux recherches en sciences de l'éducation peuvent investir les questions posées par les sciences de l'éducation. Par exemple, quid des potentialités d'émancipation de tel dispositif ou de telle forme scolaire? En quoi le processus de gestion de la classe renvoie-t-il à des questions d'ordre didactique?

Ainsi, cet allongement du questionnaire me semble permettre aux différents chercheurs, aujourd'hui, d'avancer vers des recherches plus pertinentes. D'une certaine manière, on devrait pouvoir dire que lorsqu'on fait des recherches centrées sur les pratiques d'enseignement et d'apprentissage, il y a des choses qu'on ne doit plus pouvoir ignorer, non pas parce qu'on ne serait plus «dans le coup» ou suffisamment "distingué», mais parce que le travail collectif a permis d'élaborer une certaine cartographie à partir des éléments fondamentaux de ces pratiques. Il semble que, d'une certaine manière, le processus cumulatif est beaucoup plus vivace aujourd'hui, et que par exemple, pour ce qui concerne l'étude des pratiques d'enseignement et d'apprentissage, de nouveaux standards émergent, en particulier au plan méthodologique. On prendra pour exemple les soucis méthodologiques inédits qu'ont pu introduire dans les sciences de l'éducation les didactiques, dès lors qu'elles se sont intéressées au didactique ordinaire, ou l'ergonomie, dès lors qu'elle intègre la complexité du travail professoral.

Il s'est ainsi peu à peu créé une sorte de paradigme général d'étude des pratiques, auquel s'attachent de nouveaux standards:

- la prise en compte des savoirs dans la manière dont ils orientent l'action;

- l'utilisation systématique de transcriptions de séance;

- l'utilisation systématique de méthode de réduction des données (synopsis, récit, etc.);

6. Veyne 1978 
- l'utilisation systématique de méthodes d'entretien d'analyse directe (en particulier au moyen de méthodes vidéo), par l'acteur, de ses propres pratiques;

- l'intégration systématique de questions d'ordre social, politique et éthique;

- l'effort de détermination d'invariants et de modèles de la pratique;

- la question posée de la valeur de la pratique (je reviendrai sur ce point).

Dans de tels standards, aujourd'hui, de nombreux chercheurs peuvent se reconnaître, qu'ils soient chercheurs en sciences de l'éducation, didacticiens, ou ergonomes. Une reconfiguration apparaît donc comme non seulement possible, mais déjà là.

\section{Une reconfiguration aujourd'hui?}

Une telle reconfiguration des frontières disciplinaires, à laquelle les IUFM ne sont sans doute pas étrangers, peut ainsi voir, par exemple, un didacticien du français ou des mathématiques travailler avec un ergonome et un spécialiste de l'étude des pratiques enseignantes en sciences de l'éducation: à l'allongement du questionnaire correspondant une sorte de déploiement des lieux de rencontre et des occasions de travail commun.

Au-delà de ces nouvelles opportunités, certes fructueuses pour elles-mêmes, on trouve dans certains cas l'élaboration d'un autre rapport à la pratique et d'autres modes de coopération entre enseignants et chercheurs. C'est à mon sens dans cette voie qu'il nous faut poursuivre: il y a selon moi une nouvelle épistémologie à faire vivre aujourd'hui dans la recherche en sciences de l'homme et de la société, une épistémologie de type pragmatiste, dont Dewey et Putnam pourraient être des figures.

Cette nouvelle épistémologie est en rupture avec une autre, encore majoritairement vivace dans nos communautés aujourd'hui : une épistémologie «normale», fondée sur une séparation radicale entre faits et valeurs. Au monde de la recherche, les faits; aux mondes politiques et éthiques, les valeurs. Au chercheur qui voudrait non pas seulement interroger les valeurs «de l'extérieur», mais intégrer cette question au sein de sa recherche, on dira de lui qu'il «fait de l'idéologie». Inspiré par un weberianisme commun, on fera ainsi du chercheur quelqu'un qui met à distance l'idéologie et qui évolue sur la terre ferme de l'objectivité scientifique. On pourra remarquer, sans en venir directement au pragmatisme, combien une telle vue est déjà largement battue en brèche par le travail de Pierre Bourdieu ${ }^{7}$ : l'idée d'objectivation de l'objectivation invite notamment le chercheur à prendre distance avec la posture scolastique de surplomb qui est la sienne, et l'appelle à débusquer ce qui, dans son action, est dû à des intérêts et valeurs non explicités.

Hilary Putnam a bien démontré «l'effondrement de la dichotomie entre faits et valeurs ${ }^{8}$ : tout énoncé de fait est d'une certaine manière plus ou moins pénétré de

7. Bourdieu 1992.

8. Putnam 2002. 
valeur. Telle est la thèse de l'auteur. Il ne s'agit pas de nier une distinction salutaire et commode entre les faits et les valeurs. Dans une perspective ouverte par Dewey ${ }^{9}$ et sa critique radicale des dualismes, Putnam nous met en garde contre la rigidification de cette distinction en dualisme, en dichotomie. Enregistrer l'effondrement de la dichotomie de la valeur et des faits, quelle signification cela peut-il avoir pour notre communauté de recherche? La place manque, ici, pour répondre rigoureusement à cette question, mais un premier élément de réponse pourrait être celui-ci: il faudrait probablement penser à nouveaux frais des dispositifs de coopération entre chercheurs et professeurs. Pour le chercheur, il ne s'agit plus maintenant de continuer à consacrer une division du travail héritée qui le fait encore, d'une manière ou d'une autre, homo academicus surplombant, dans une posture se délectant de temps à autre de la déploration critique. Un point important, ici, est le suivant: renoncer à la posture d'homo academicus ne signifie pas pour autant investir la posture d'acceptation benoîte - anything goes - qu'il n'est pas rare, il me semble, de voir se développer en lieu et place de la posture scolastique. On trouve là, en effet, une confusion possible tout à fait intéressante, qu'on peut décrire sous deux avatars différents :

- on veut quitter la posture surplombante, et, un peu à la manière de l'ethnométhodologie radicale, on va trouver dans les comptes rendus des membres, l'alpha et l'omega du travail de recherche;

- on produit ce travail de recherche sans prise en compte de la valeur de la pratique, ce qui fait qu'on peut voir dans certains cas des thèses entières construites sur des pratiques que la guilde des professeurs, si elle existait, tiendrait comme indigentes, sans que jamais le chercheur ne (se) pose la question de ce qui est réellement enseigné et appris. Au passage, on verra bien là une nouvelle conséquence de la dichotomie des faits et des valeurs: je suis un chercheur, et je n'ai donc pas à juger.

Il me semble donc qu'un tournant décisif, aujourd'hui, pour les recherches en éducation, pourrait consister à construire avec les professeurs des dispositifs d'enseignement dont la responsabilité soit à la fois clairement située et pleinement partagée. Ce pourrait être l'un des objectifs majeurs des IUFM intégrés à l'Université.

Plus largement, il me semble que la question posée aux sciences de l'éducation est celle de leur utilité et, dirais-je, de leur valeur: il me paraît que le mouvement que je viens de décrire, et qui renoue, me semble-t-il, avec certaines des intuitions et des projets du début, peut permettre d'envisager à nouveau frais la question de la valeur. Gilles Deleuze disait à la fin de sa vie qu' «il faut en finir avec le jugement». Je m'associerai tout à fait à ce souhait, et aimerais que les sciences de l'éducation s'y associent également.

Mais en finir avec le jugement, ce n'est pas en finir avec la valeur, avec les vertus, c'est en finir avec la division sociale qui fait les uns juges et les autres jugés, c'est

9. Dewey 1920-2003. 
construire, par exemple pour ce qui concerne les institutions didactiques, des dispositifs dont la valeur tient aux fins que professeurs et chercheurs élaborent ensemble, et évaluent ensemble. Là, dans ce travail complexe de traduction réciproque de leur point de vue et de leur expérience, là peut résider à mon sens une valeur première et une utilité cardinale des sciences de l'éducation.

Gérard SENSEVY

CREAD

Université de Haute Bretagne - Rennes II

\section{Références bibliographiques}

Bourdieu P. (1992), Réponses: pour une anthropologie réflexive, Paris, Seuil.

Chevallard Y. (2007), «Éducation et didactique: une tension essentielle», Éducation et didactique, vol. 1, n 1, p. 9-28.

Dewey J. (1920-2003), Reconstruction en philosophie, Pau, Farrago - Léo Scheer.

LAHIRE B. (2007), « La sociologie, la didactique et leurs domaines scientifiques », Éducation et didactique, vol. 1, n 1 , p. 73-82.

LIPING MA (1999), Knowing and Teaching Elementary Mathematics: Teacher's Understanding of Fundamental Mathematics in China and the United States, Mahwah, Lawrence Erlbaum.

Putnam H. (2002), Fait / valeur: la fin d’un dogme, Paris, Éditions de l'Éclat.

Schubauer-Leoni M.-L., Leutenegger F., Forget A. (2007), «L'accès aux pratiques de fabrication de traces scripturales convenues au commencement de la forme scolaire: interrogations théoriques et épistémologiques", Éducation et didactique, vol. 1, n 2, p. 9-36.

Sensevy G., Mercier A. (dir.) (2007), Agir ensemble. L'action didactique conjointe du professeur et des élèves, Rennes, Presses universitaires de Rennes.

Shulman L. (2007), "Ceux qui comprennent. Le développement de la connaissance dans l'enseignement », Éducation et didactique, vol. 1, n 1, p. 97-114.

Veyne P. (1978), Comment on écrit l'histoire, Paris, Seuil. 



\section{NOUVEAUX TERRAINS, NOUVEAUX TERRITOIRES}





\section{PRÉSENTATION : \\ DE NOUVELLES DEMANDES SOCIALES \\ ET DE NOUVELLES PROBLÉMATIQUES DE RECHERCHE}

Nouveaux terrains ou nouveaux territoires: de nouvelles demandes sociales à de nouvelles problématiques de recherche. Questions vives, assurément, pour l'ensemble des domaines professionnels concernés par les deux communications qui suivent: la formation d'enseignants et la formation des travailleurs sociaux; domaines pour lesquels sont examinées les correspondances entre champs de pratiques et champs de recherche.

Les champs de pratiques désignent l'activité des professionnels d'un domaine, spécialistes, parfois diplômés et souvent compétents; celles aussi de chercheurs de divers statuts, spécialités, références et renommées; celles encore du monde politicoadministratif de différents niveaux, plus ou moins proches des centres de décision ou des opérateurs au quotidien: services centraux, chefs d'établissement, responsables de formation, etc. Les champs de recherche se découpent suivant les limites des domaines professionnels observés et analysés, mais aussi suivant des logiques de spécialités (économie, sociologie, histoire, science politique, didactiques, etc.); suivant les objets découpés dans les réalités sociales et les échelles que privilégient ces découpages (les individus, les groupes, les établissements, les organisations, les gouvernements, etc.); les paradigmes et les références; les rapports des chercheurs à l'objet et aux sujets enquêtés; leurs rapports avec l'éventuel commanditaire ou financeur, etc. Évidemment, avant tout cela, faudra-t-il encore avoir discuté ce que recouvre, pratiquement, la notion de recherche, d'activité ou d'action de recherche.

Tissant ces éléments épars et hétérogènes, de façon lacunaire et inégale, il y a la potentialité d'une «demande sociale» au pluriel, en mouvement, en débat; plus sûrement une réalité de finalités sans doute plurielles, qui sous-tendent toutes les situations, allant de la commande politique à l'absence de mobile autre que professionnel à l'échelon individuel.

On connaît par ailleurs la charge d'enjeu social, épistémologique, politique, qui connote l'usage de la notion de terrain. Notion-enjeu d'un rapport de pouvoir autour de la question de la maîtrise de la pratique et de la formation professionnelle, y compris celle des chercheurs dans leurs disciplines respectives et autour de leurs objets quelquefois partagés. Un usage assez dispendieux en est généralement fait pour revendiquer pour soi (individu, groupe, établissement, administration) la 
formation efficace et discréditer, ou ne pas reconnaître qu'il y a chez l'autre, variant suivant les contextes, du savoir opérationnel, de l'efficacité, des capacités d'amélioration de l'action et du système. Si la notion de terrain doit être associée à celle d'enjeu, de confrontation entre des modèles concurrents de professionnalité, de formation professionnelle et de production de savoir finalisée par l'amélioration de l'action, alors sans doute, le terrain a-t-il tendance à se déplacer de lieux qui structuraient les débats tout en étant parfois violemment attaqués, vers les opérateurs eux-mêmes. C'est peut-être à l'échelon de l'individu qu'aujourd'hui se discutent et se déterminent, entre établissements et collectifs, institutions et expériences, les choix professionnels, modèles de professionnalité et décisions dans l'action.

Quant au territoire, le géographe en connaît les tensions entre deux acceptions, celle de l'espace borné-contrôlé, sens le plus ancien d'espace correspondant à la logique de l'État avec son exhaustivité et ses frontières externes; celle d'espace approprié, c'est-à-dire d'espace disposant d'un attribut d'appartenance ou d'identification. Le territoire est dans ce deuxième cas la composante identitaire de tout espace. On voit bien alors comment, d'un côté, un groupe institutionnel, professionnel ou autre, peut se réclamer seul occupant légitime ou seul responsable d'un territoire (celui de la pédagogie dans un établissement, celui des compétences professionnelles dans une académie, celui d'une revue scientifique dans une spécialité, etc.) et disputer, à la faveur d'un changement opéré à une autre échelle, les positions d'un autre groupe. Dans le même temps, nombre d'individus, à la croisée des mondes que nous évoquons, ont pourtant à se positionner dans plusieurs de ces territoires, en rapport avec leurs propres champs de pratiques. Concrètement, ils font fonctionner des réseaux d'information et d'influence qui ouvrent et parcourent ces différents champs. De quels territoires se sentent-ils alors? Quelles territorialités se fabriquent ainsi à l'articulation des champs de pratique de la formation, de la recherche sur et de l'encadrement de la formation? Sous quelles conditions de statut, de reconnaissance du travail, d'autonomie des différents niveaux et périmètres d'action, des territorialités pluriréférentielles peuvent-elles se construire? Estce que les individus sont condamnés à la "gestion» au quotidien des tensions que produit la pluriactivité dans des établissements et des institutions de finalité et de statut différents? Est-ce que les modèles du néo-management sont le seul horizon référentiel pour penser et prévoir les changements dans les organisations et dans l'activité ? Comment construire des accords entre intervenants à des titres divers dans des dispositifs hybrides par les niveaux d'intervention, les statuts et les fonctions des personnes (le conseil d'école d'un institut de formation, le conseil de direction d'un organisme académique de formation d'adultes, le conseil d'administration d'un établissement, etc.)?

Comme observateur et familier à chaque fois d'un petit nombre de ces processus complexes qui lient les décisions politiques relatives à la formation à leurs effets chez les usagers des services, en passant par les médiations institutionnelles, professionnelles et personnelles, chacun devine que les modèles simples de la linéarité (conception / application) et du puzzle (diffusion de l'innovation et du changement 
par le maillage territorial d'État) ne sont pas opératoires pour décrire ce qui se passe, même si ces modèles inspirent un certain nombre de décisions.

Les mondes de la formation, de la recherche et du politique en relation avec la formation sont autonomes autant qu'ils sont composites. Chacun pourrait fournir rapidement une illustration du modèle des cités de Luc Boltanski et Laurent Thévenot à partir de situations qu'il connaît. Bien entendu, les petits mondes des recherches en éducation et en formation ne sont pas seulement structurés par les valeurs, les attitudes et les objets de la cité inspirée (la création). Les cités domestiques (la famille, la tradition) et marchandes (les «lois» de la concurrence) y règlent aussi bien des décisions. Ces mondes de la formation sont aussi poreux : les références de la performance propre à la cité industrielle et de la renommée propre à la cité de l'opinion envahissent la sphère politique de la formation et de la recherche. Ils sont aussi intelligents, au sens où ils produisent des savoirs légitimes et des décisions argumentées. Nombre d'acteurs intermédiaires, y compris des chercheurs, à des échelons territoriaux médians, s'essaient à des articulations, à des hybridations qui tentent de ne pas sacrifier l'intérêt collectif de la cité civique à toute autre référence exclusive. Enfin, des individus bien réels se trouvent alternativement dans ces différents mondes, travaillant ici ou ailleurs à différents postes et fonctions: enseignant, formateur, administrateur, chercheur, conseiller, évaluateur, etc.

C'est donc qu'il nous faut choisir de mieux décrire, de mieux comprendre les médiations, les altérations, les circulations entre ces mondes, leurs «véhicules» (formation, stratégie de recrutement, politique de communication, mots-enjeux, etc.), leurs réseaux (fine circulation d'information professionnelle et injonction institutionnelle massive et répétée), leurs «cibles» (identités, conceptions, processus de décision, gestes, structures, etc.); mieux comprendre aussi comment, parfois, se réalisent des sauts d'échelle qui permettent à certaines pensées, certains modèles de la formation (et donc du social, de l'humain et du politique) de devenir des références au-delà des premiers cercles ou, au contraire, de perdre une suprématie dont ils venaient d'écrire l'histoire de son irrésistible ascension. 



\section{LA FORMATION DES ENSEIGNANTS}

Résumé: La demande sociale en direction de la formation des enseignants doit être pensée en fonction de ses différents contextes ou registres : évolution des pratiques d'éducation, des normes et des valeurs, orientations et exigences des politiques scolaires, représentations du métier. Après avoir rappelé qu'il y a une longue tradition de la recherche dans ces différents domaines, l'auteur souligne qu'il serait souhaitable de mieux articuler les savoirs formalisés issus de la recherche aux compétences professionnelles requises dans les classes et de s'ouvrir aux recherches similaires dans les secteurs professionnels voisins.

Mots clés: formation des enseignants, demande sociale, recherche en éducation, compétence professionnelle, politique éducative.

\section{De «nouvelles demandes sociales», de nouveaux métiers}

Concernant la formation des maîtres, on constate tout d'abord l'ancienneté des "demandes sociales", si tant est que l'on en ait trace ou que des débats expriment d'une certaine façon de telles demandes. Ainsi, au début du XIX ${ }^{\mathrm{e}}$ siècle, la question du type de structures de formation des maîtres (Écoles normales, écoles mutuelles, écoles modèles ${ }^{1}$ ) renvoie à des enjeux économiques mais plus encore idéologiques et sociaux, concernant le développement de la scolarisation élémentaire, la place de l'école et de l'enseignant dans la communauté, les instances de contrôle de la formation, les modèles de l'exercice professionnel souhaitable, etc. À la fin du siècle, les débats sur les dangers de «l'esprit primaire » ${ }^{2}$ qu'auraient favorisé les Écoles normales poseront la question du recrutement de bacheliers issus des lycées plutôt que du réseau des écoles primaires supérieures; mais, dans un contexte politique de luttes idéologiques exacerbées autour de la question des congrégations enseignantes en 1904 et de la séparation de l'Église et de l'État en 1905, le pouvoir républicain s'appuie sur l'ordre primaire qu'il ne saurait affaiblir.

1. Grandière 2006.

2. A. Massé, député, rapport de la commission du budget, séance du 13 juillet 1905. La culture «primaire» inculquée par les Écoles normales a pour effet, selon les débats de l'époque, d'enfermer les élèves-maîtres dans un monde coupé des réalités du terrain professionnel et de la vie sociale, fait de certitudes simplistes et d'intolérance, terreau favorable à la diffusion des idées pacifistes et socialistes. 
On pourrait multiplier les exemples de «nouvelles demandes sociales de formation » à différentes époques. Ils autorisent quatre remarques.

1) La demande sociale concernant la formation des maîtres n'a de sens que référée à divers niveaux contextuels, en particulier :

- celui des évolutions sociétales. Ainsi les rapports éducatifs au sein de la famille et de l'école se sont profondément modifiés: sont régulièrement mises en avant la crise du rapport à l'autorité et à la norme, les incertitudes quant aux valeurs éducatives, les difficultés pour certains élèves à accorder une valeur et un sens cognitifs aux activités d'apprentissage et aux contenus des savoirs scolaires;

- celui des politiques scolaires, dans leurs dimensions de réformes de la scolarisation (prolongation généralisée, massification de l'enseignement secondaire), ou de nouvelles missions de l'école (hausse de la qualification de l'ensemble de la population, formation d'un capital humain correspondant aux nouvelles exigences du marché du travail, développement de la cohésion et de l'intégration sociale, de la justice et de la citoyenneté, etc.).

2) La demande sociale concernant la formation des maîtres nous renvoie également à l'analyse de la structuration et de l'organisation actuelle de l'institution, en termes de décentralisation et de déconcentration («territorialisation des politiques d'éducation »), de pilotage stratégique de l'institution et de modernisation de l'État (professionnalisation des acteurs et nouveau management). Si, de 1945 à 1975, les budgets de l'Éducation nationale sont multipliés par neuf en francs constants, ils stagnent à partir du milieu des années 1970: la gestion de l'institution et l'évaluation de son efficacité viennent au-devant de la scène et le discours gestionnaire met en avant la qualité, l'efficience, la mobilisation des acteurs autour de projets, etc.

3) La demande sociale concernant la formation des maîtres est aussi imprégnée par les conceptions que l'on a du métier auquel on doit former, les modèles d'excellence de l'exercice professionnel (un homme cultivé, un humble instituteur républicain, un éducateur d'adolescents, un praticien réflexif, un spécialiste de l'ingénierie ou des relations humaines) et les connaissances que l'on a des conditions d'exercice (hétérogénéité des élèves, élargissement du cadre de référence de l'action pédagogique, par exemple). En d'autres termes, la demande sociale porte une / des conception(s) de la normalité du métier aujourd'hui et de ce qui est rejeté à sa périphérie.

4) Cependant, la demande sociale n'est pas unifiée: les attentes ne font pas consensus, pas plus aujourd'hui qu'hier. Si on observe un relatif consensus sur la nécessité d'adapter les métiers enseignants à la nouvelle donne de la scolarisation, il n'y a pas accord sur le modèle du "praticien réflexif», encore moins sur la politique de professionnalisation menée par l'institution. De même, les enseignants débutants sont pris dans une tension entre les modèles professionnels préconisés en formation et l'effet terrain, la mémoire héritée valorisée par les pairs et les difficultés de la prise de fonction.

La demande sociale de formation est donc une construction historique et, en tant que telle, doit être analysée en termes d'enjeux sociétaux contextualisés, de modèles de professionnalité (d'excellence), de stratégies de groupes d'acteurs, etc. 


\section{De nouvelles problématiques de recherche?}

Il a souvent été relevé que les structures et dispositifs de formation mis en place par l'institution "précèdent» le développement de la recherche qui, dans cette sorte de configuration, aurait pour fonctions principales la compréhension critique et réflexive des politiques et des dispositifs, la compréhension des interactions entre les acteurs, voire parfois l'étayage de la décision ${ }^{3}$. Selon A. Prost ${ }^{4}$, la recherche a lentement pris conscience de la complexité des processus de formation. Durant les années 19501960, deux positions antagonistes dominaient, issues de traditions que l'on peut faire remonter aisément jusqu'à la fin du XIX ${ }^{\mathrm{e}}$ siècle: l'une proclamait l'inutilité de la formation (la leçon d'agrégation passait dans certains rapports de concours pour le modèle de l'excellence professionnelle), l'autre tentait de construire l'exercice professionnel comme des applications de la psychologie de l'enfant et de l'apprentissage, les cours de psychologie et de psychopédagogie constituant les fondements et l'épine dorsale de la formation des maîtres. Un certain nombre de déplacements s'opéreront au sein même de la formation des maîtres, centrée dorénavant sur l'apprentissage d'un métier, la nécessité d'une compréhension réflexive des actes et des interactions professionnels : citons les réformes du CPR (Centre pédagogique régional) au début des années 1980 pour le second degré, la longue émergence des dispositifs d'analyse des pratiques dans les Écoles normales au cours des années 1970-1980, la mise en place bien avant la naissance des IUFM (Institut universitaire de formation des maîtres) d'un mémoire professionnel dans les Écoles normales nationales d'apprentissage pour les professeurs de l'enseignement professionnel.

On rappellera qu'il existe une longue tradition de recherche en ces domaines, prenant des formes et des objets variés. L'Association des enseignants et chercheurs en sciences de l'éducation ${ }^{5}$ propose de distinguer différents types de travaux, dont trois sont présents très tôt dans le champ éducatif: les recherches philosophiques qui produisent des savoirs dans une logique de réflexion critique et de questionnements sur les valeurs et les finalités; les recherches dites «fondamentales» avec problématique, méthodologie, recueil d'un corpus de données; les recherches praxéologiques qui, plus proches des terrains et des acteurs, proposent des outils, des ingénieries, mettent en place une innovation pédagogique, etc. Disciplines philosophiques et recherches expérimentales occuperont massivement la recherche en éducation depuis la fin $\mathrm{du} \mathrm{XIX}^{\mathrm{e}}$ et au cours de la première moitié du $\mathrm{XX}^{\mathrm{e}}$ siècle $^{6}$. Recherches actions, expérimentations, innovations dites "contrôlées» se multiplieront dans le dernier tiers du XX ${ }^{\mathrm{e}}$ siècle.

Qu'en est-il des recherches aujourd'hui ? On trouve bien évidemment des tentatives pour décrire et interroger les grandes tendances du développement de la recherche

\footnotetext{
3. AECSE 2001, 71.

4. Prost 2002, 37.

5. AECSE 2001, 69.

6. Dottrens \& Mialaret 1969; Vigarello \& Hermine 1994; Depaepe 1998; Hoffstetter \& Schneuwly 2002.
} 
depuis les années 1980 dans les (co-)publications de l'AECSE ${ }^{7}$. Selon B. Charlot ${ }^{8}$, deux grands courants se développent dans les années 1980: d'une part l'ingénierie de la formation, le management éducatif, la recherche sur les pratiques efficaces, en privilégiant les approches didactiques et de psychologie cognitive; d'autre part les recherches compréhensives sur les situations, les pratiques, les acteurs. A. Prost relève cependant, malgré les efforts de l'AECSE et en son temps ceux du Comité national de coordination de la recherche en éducation (CNCRE), la difficulté de procéder à un état des lieux et le manque de coordination des recherches ${ }^{9}$, en partie parce que les recherches sont fortement contextualisées et en conséquence dispersées et d'une extension limitée. En termes de problématiques, on observe un éclatement des recherches sur la formation des maitres qui s'élargissent à toutes les dimensions du développement professionnel: les dispositifs de formation, les pratiques et l'exercice professionnel stricto sensu, les phases de la carrière, les transformations des cultures et des identités, les formes historiques d'organisation sociale du travail, le contrôle du travail, la reconnaissance et le statut des acteurs, etc.

\section{Des ouvertures pour conclure}

On se bornera à esquisser trois pistes, qui sont autant de vœux, pour conforter les développements des recherches sur la formation dans leurs rapports aux demandes sociales actuelles quant à la formation des maîtres.

L'un des objectifs de l'universitarisation des formations professionnelles est d'articuler les savoirs formalisés issus des recherches et les compétences mises en œuvre par les praticiens dans les classes. On sait que les savoirs professionnels des enseignants ne constituent pas un corps de connaissances homogène et stabilisé et l'on observe un certain éclatement de la recherche sur ces thèmes tant la question de la nature des savoirs enseignants reste complexe ${ }^{10}$. Cette dispersion et cette complexité ne suffisent pas cependant à expliquer le fait que la crédibilité ou l'expertise des groupes professionnels enseignants n'est assurée ni au niveau des décideurs ni au niveau de l'opinion publique: ainsi les débats récurrents sur les méthodes de lecture ou sur la maîtrise de l'orthographe réduisent l'expertise et les pratiques au seul choix de méthodes. Il serait souhaitable de développer un jour les recherches (historiques et sociopolitiques) sur la construction d'une (non-)reconnaissance de l'expertise enseignante: quels sont les acteurs concernés (e.g. associations d'enseignants, chercheurs, experts ministériels, politiques), les réseaux constitués autour de ces questions, les positions des uns et des autres, etc.

\footnotetext{
7. AECSE 1993-2001; Jeannel et al. 1994; Charlot 1995.

8. Dans Jeannel et al. 1994.

9. Prost 2002, 24.

10. Lessard et al. 2004 .
} 
Les recherches gagneraient également à ne pas considérer leur objet isolément, à le «désenclaver» en prenant en compte les rapports entre les groupes professionnels enseignants, les autres acteurs au sein de l'institution (experts, corps d'inspection, personnels de direction, formateurs), les partenaires éducatifs, le public, mais aussi les politiques éducatives européennes et les forces du marché, en particulier les formes diverses par lesquelles les grands groupes de l'édition multimédia investissent le domaine éducatif.

Elles doivent enfin impérativement s'enrichir des travaux concernant des groupes professionnels voisins confrontés à des exigences de mutation du même ordre (secteur santé, paramédical, métier du secteur social, police par exemple) et de façon plus fréquente des recherches internationales sur le sujet.

Vincent LANG

CREN

Université de Nantes

\section{Références bibliographiques}

Aecse (1993-2001), Les sciences de l'éducation. Enjeux et finalités d'une discipline, Paris, INRP.

Charlot B. (1995), Les sciences de l'éducation, un enjeu, un défi, Paris, ESF.

Depaepe M. (1998), «La recherche expérimentale en éducation de 1890 à 1940 : les processus historiques sous-jacents au développement d'une discipline en Europe de l'Ouest et aux États-Unis», in Le pari des sciences de l'éducation, R. Hofstetter, B. Schneuwly (éd.), Bruxelles, De Boeck, p. 29-56.

Dottrens J., Mialaret G. (1969), «Le développement des sciences pédagogiques et leur état actuel», in Traité des sciences pédagogiques, M. Debesse, G. Mialaret (dir.), Paris, PUF, vol. 1, p. 19-73.

Grandière M. (2006), La formation des maîtres en France, 1792-1914, Paris, INRP.

Hofstetter R., Schneuwly B. (2002), Science(s) de l'éducation (XIX ${ }^{e}-X X^{e}$ siècles). Entre champs professionnels et champs disciplinaires, Berne, Peter Lang.

Jeannel A., Clanché P., Debarbieux E. (dir.) (1994), 25 ans de sciences de l'éducation. Bordeaux, 1967-1992, Paris, AECSE - INRP.

Lessard C., Altet M., Paquay L., Perrenoud P. (dir.) (2004), Entre sens commun et sciences humaines. Quels savoirs pour enseigner?, Bruxelles, De Boeck.

Prost A. (2002), Pour un programme stratégique de recherche en éducation, Rapport aux ministres de l'Éducation nationale et de la Recherche, Paris, La Documentation française.

Vigarello G., Hermine M. (1994), «L'histoire des sciences de l'éducation en France», in Pour les sciences de l'éducation. Approches franco-québécoises, M. Bernard (dir.), Paris Montréal, INRP - Revue des sciences de l'éducation. 



\section{LE TRAVAIL SOCIAL ET LA FORMATION DES TRAVAILLEURS SOCIAUX}

Résumé: Depuis une dizaine d'années, les transformations du champ et des pratiques du travail social ont été si nombreuses que l'on peut parler d'une véritable recomposition de cet univers professionnel. Pour l'essentiel, les évolutions concernent les cadres et les modalités de la formation et de la professionnalisation des travailleurs sociaux. L'article se propose d'identifier les transformations en cours, en rappelant les principales caractéristiques de l'appareil de formation tel qu'il existe. Il s'agira ensuite de décrire les évolutions et d'analyser l'impact que fait peser la décentralisation sur le domaine, la rénovation des diplômes et les conséquences sur les formations initiale et continue des travailleurs sociaux de l'introduction, avec la VAE, de nouveaux référentiels professionnels. Enfin, la redéfinition des qualifications et des diplômes pose le problème des relations ave les recherches universitaires dans le champ de la formation, en particulier avec celles qui sont menées au sein des sciences de l'éducation.

Mots clés: intervention sociale, travailleurs sociaux, formation, professionnalisation, sciences de l'éducation.

Dans la reconfiguration en cours, ce sont donc les cadres et les processus de la formation et de la professionnalisation qui sont appelés à évoluer. Aussi, après avoir identifié les transformations du champ, on rappellera brièvement les principales caractéristiques de l'appareil de formation des travailleurs sociaux et l'on cherchera à situer les transformations à l'œuvre au niveau de la formation initiale et continue des professionnels de l'intervention sociale: impact de la décentralisation des formations sociales, rénovation des diplômes et apparition de référentiels induits par la VAE... Enfin, dans un contexte où les relations avec l'Université sont interrogées de façon récurrente, quels peuvent être les apports des sciences de l'éducation sur les plans de la recherche et de la formation?

\section{Les travailleurs sociaux}

De plus en plus utilisée à partir du milieu des années 1960, l'expression «travail social» n'est pas la traduction littérale de l'anglais «social work», les "social workers» ne correspondant qu'aux assistants sociaux en France, mais permet de désigner un ensemble hétérogène d'activités multiples mises en œuvre en direction de populations en difficulté par une grande diversité de professionnels : "les travailleurs sociaux». 
Intégrés auparavant dans l'ensemble des "professions sanitaires et sociales", les travailleurs sociaux - des femmes pour la plupart, voire quasi exclusivement selon la catégorie de professionnels considérée - exercent dans des secteurs extrêmement diversifiés (pauvreté, handicap, délinquance, protection de l'enfance, aide à domicile...), auprès de populations multiples mais en butte à des difficultés suffisamment sérieuses pour justifier leur intervention. Historiquement, le développement des professions sociales s'est étayé sur celui des professions de santé: les infirmières visiteuses sont devenues progressivement assistantes sociales et ce sont des psychiatres d'exercice universitaire qui sont à l'origine du secteur de l'enfance inadaptée et de l'émergence du métier d'éducateur... Aujourd'hui encore, la proximité des deux ensembles est telle, surtout si l'on se réfère à la notion de soin dans son acception anglaise de care et non de cure, que l'on a du mal à distinguer ce qui sépare les activités d'une aide-soignante de celles d'une aide médico-psychologique (AMP), lorsque toutes deux peuvent intervenir ensemble auprès de personnes handicapées par exemple.

L'ensemble intitulé «travailleurs sociaux» correspond, en France, au regroupement, opéré à partir du début des années 1970, de toute une série de "professions sociales et éducatives» jusque-là différenciées et cloisonnées, dont l'essor, lié à la tertiarisation de l'économie et à l'émergence d'une "société de service " ${ }^{1}$, s'est révélé spectaculaire depuis trente-cinq ans. Alors qu'on distinguait traditionnellement trois grandes catégories de «métiers administrativement reconnus ${ }^{2}$ : les métiers de l'aide (autrefois «de l'assistance»), les métiers éducatifs et enfin ceux de l'animation, une étude plus récente ${ }^{3}$ dénombre 600 ooo travailleurs sociaux en 2002 - soit une augmentation de $40 \%$ en 10 ans - regroupés en quatre catégories de métiers du travail social:

- 51500 professionnels de l'aide sociale (hors aide à domicile ${ }^{4}$ ), dont 40400 assistants de service social et 4600 conseillers en économie sociale et familiale;

- 177100 professionnels de l'éducation spécialisée: 3300 chefs de service, 99100 éducateurs spécialisés, 12800 éducateurs de jeunes enfants, 10100 éducateurs techniques et éducateurs techniques spécialisés, 17700 moniteurs éducateurs, 9500 moniteurs d'ateliers, 24700 aides médico-psychologiques; sans intégrer les éducateurs du ministère de la Justice (Protection judiciaire de la jeunesse) ainsi que certains enseignants spécialisés qui exercent également des fonctions éducatives;

- 49700 professionnels de l'animation;

- et 308000 professionnels de l'accueil d'enfants à leur domicile (dont 250100 assistantes maternelles).

\footnotetext{
1. Laville 2005.

2. Woitrain 2000 .

3. Beynier et al. 2005 .

4. Bressé 2004.
} 
L'appartenance de certaines catégories de professionnels aux métiers du travail social peut être discutée: c'est, par exemple, le cas des assistantes maternelles dont le statut a été réformé (loi du 27 juin 2005). On distingue dorénavant les 250100 assistantes maternelles qui accueillaient, en 2002, 740000 enfants de moins de 6 ans à la journée, des 58200 assistantes maternelles à titre permanent, nouvellement appelées «assistantes familiales », qui accueillaient 65000 enfants dans le champ de la protection de l'enfance. On pouvait contester le statut de travailleur social aux premières en raison d'une faible qualification, mais pas aux secondes. On s'interrogera également sur l'homogénéité des professionnels de l'animation, écartelés entre un secteur de diffusion artistique (Maisons de la culture, Centres d'action culturelle...), le développement d'activités sportives et de loisir de plus en plus spécialisées, et des modes d'action relevant de l'intervention sociale. Par ailleurs, considérera-t-on prochainement les médiateurs familiaux (diplôme d'État instauré fin 2003) comme une nouvelle catégorie de travailleurs sociaux?

Les travailleurs sociaux ont pour principaux employeurs l'État, les collectivités territoriales et surtout de très nombreuses associations gestionnaires d'établissements et de services, mais selon une répartition spécifique en fonction de la catégorie de professionnels considérée. Si un premier repérage peut être effectué en fonction de la sphère d'activités professionnelles et du type d'organisme de rattachement, il convient de tenir compte, secondairement, du niveau de qualification reconnu pour l'accès à ces métiers, selon la formation initiale dispensée et le diplôme obtenu (niveaux V, IV, III...). Ainsi distingue-t-on habituellement trois niveaux:

- les professions en contact direct avec les usagers, nécessitant une qualification de niveau $\mathrm{V}$ ou IV et dont l'essor quantitatif s'est révélé le plus important ces dernières années (front office);

- les métiers traditionnels, de niveau III, d'accompagnement éducatif et social, en faible augmentation et tenus de plus en plus éloignés des usagers dans des activités de gestion des dossiers et de coordination des actions (back office);

- les professionnels d'encadrement et de direction de niveaux II et I, naguère promus à l'ancienneté parmi des diplômés de niveau III, même si on semble leur préférer dorénavant de jeunes titulaires de DESS et, aujourd'hui, de masters de droit ou de gestion.

À la segmentation en secteurs d'activités s'ajoute une forte hiérarchisation des formations et des diplômes. Il s'agit donc d'un champ professionnel peu homogène et aux frontières mouvantes au sein duquel chaque catégorie a connu un processus de professionnalisation spécifique et différencié dans le temps. De plus, ces données chiffrées ne constituent qu'une photographie partielle ne prenant pas en compte les «nouveaux métiers du social », regroupés avec les travailleurs sociaux dans ce que l'on appelle désormais «les professionnels de l'intervention sociale», ni les professionnels relevant d'autres ministères de tutelle (Éducation nationale, Justice...). 


\section{Du travail social à l'intervention sociale}

Jusqu'au début des années 1980, c'est une logique de «ciblage» qui a animé le mode de structuration et de développement du travail social: définition d'une population à problème à laquelle on s'efforce de faire correspondre une compétence professionnelle, voire un traitement institutionnel spécifique, chaque métier traditionnel du travail social s'articulant autour de plusieurs dimensions:

- une assise institutionnelle liée au type d'établissement, d'équipement ou de service, dans lequel on exerce (institut médico-éducatif, centre social, club de prévention...);

- un type de public, une population spécifique, en direction de laquelle le travailleur social intervient (handicapés, déficients, jeunes en danger...);

- une qualification, conférant une certaine professionnalité par l'usage de techniques spécifiques plus ou moins efficaces.

En tant qu'acteurs de la relation d'aide, les travailleurs sociaux constituent assurément un groupe professionnel, mais segmenté et aux qualifications spécifiques.

Plusieurs facteurs ont contribué à la transformation du paysage institutionnel à tel point que certains auteurs ont pu avancer l'hypothèse de la fin du travail social ${ }^{5}$. Montée de l'exclusion appelant à poser en d'autres termes «la question sociale», impact de la décentralisation conférant une place déterminante aux collectivités territoriales dans la définition des politiques sociales, développement de politiques transversales (politique de la ville, prévention de la délinquance, insertion par l'économique...), développement de nombreuses initiatives bénévoles en direction de publics en difficulté... Sous l'effet conjugué de ces facteurs, plusieurs mouvements sont repérables simultanément en matière d'emploi dans le secteur social:

- apparition de nouveaux postes de travail qualifiés (chargés de mission, chefs de projet, agents de développement, formateurs d'insertion...) liés à l'émergence de nouveaux dispositifs institutionnels;

- développement d'emplois peu qualifiés, le plus souvent occupés par des jeunes en difficulté d'insertion, pour assurer des activités de médiation au contact de publics diversifiés;

- augmentation de postes d'encadrement, notamment dans les services sociaux départementaux, après la décentralisation, davantage occupés par des titulaires de diplômes de management (droit, sciences économiques...) que par des professionnels issus des métiers traditionnels du travail social; d'où l'opposition entre «un social d'intervention» et un «social de gestion».

Aussi, quand il a été question d'identifier les évolutions dans le secteur social, à la fin des années 1990, les chercheurs ont-ils choisi de recourir à l'expression «intervenants sociaux", plus globalisante que celle de "travailleurs sociaux», qui désigne

5. Ion 1998. 
les métiers traditionnels du social; en formulant l'hypothèse que les premiers étaient davantage recrutés en fonction de leur compétence, alors que c'est une qualification qui procurait leur assise aux seconds ${ }^{6}$. En effet, selon une logique de qualification professionnelle, les salariés sont recrutés sur la base d'un statut défini par des conventions collectives et dont l'acquisition repose sur l'obtention d'un diplôme national. La logique de la compétence est davantage d'ordre individuel, puisque recrutement, salaire et position dans l'organisation sont fonction de l'expérience, du parcours et des acquis professionnels de la personne.

\section{La professionnalisation des intervenants / travailleurs sociaux en débat}

Le débat qualification / compétence ${ }^{7}$ a sans doute contribué à relancer en partie celui de la formation des intervenants / travailleurs sociaux, compte tenu de l'émergence de nouveaux besoins sociaux et d'une pyramide des âges vieillissante, tout aussi préoccupante que celle des professionnels de l'enseignement et de la santé.

L'appareil de formation apparaît pour le moins morcelé: 313 établissements, 348 sites et 676 sections de formation. En dépit de cette dispersion et de ce cloisonnement, l'heure est au regroupement et à la mise en place de passerelles, dans un contexte institutionnel de décentralisation accentuée qui n'a pas été sans provoquer des inquiétudes. La loi du 13 août 2004 («acte II de la décentralisation») a organisé à partir du $1^{\text {er }}$ janvier 2005 le transfert du financement des centres de formation professionnelle aux conseils régionaux. Quel fonctionnement adopter dans un système où l'État décide des diplômes et de l'architecture des formations alors que la Région encadre l'organisation, la planification et le financement de ces formations? Schéma national puis schémas régionaux des formations du social, augmentation des quotas d'admission en centres de formation, développement de nouvelles formules de formation, de certification et d'accès à l'emploi (apprentissage, validation des acquis de l'expérience...), autant de dispositifs censés endiguer le déficit prévisible de professionnels, à défaut de promouvoir leur professionnalité ${ }^{8}$.

\section{La formation professionnelle initiale}

Qu'il s'agisse des formations initiales ou des formations continues et supérieures, le mode de professionnalisation privilégié est celui de l'alternance, combinant cours et apports de savoirs en centres de formation (pour la plupart de gestion associative) et formation pratique avec des stages sur le terrain. D'où la multiplicité des possibilités de formation: voie directe, en situation d'emploi, apprentissage...

\footnotetext{
6. Chopart 2000.

7. Chauvière \& Tronche 2002.

8. Grenat 2006.
} 
Le système de formation initiale est organisé sur le mode de la reproduction: responsables de centres de formation, «formateurs permanents", "formateurs terrain» (autrefois «moniteurs de stage»)... sont pratiquement tous des professionnels ou d'anciens professionnels. Deux aspects caractérisent, en outre, la formation initiale professionnelle des intervenants / travailleurs sociaux:

- une inscription dans l'ensemble extra-universitaire, même si certaines formations (assistant de service social, éducateur spécialisé, gestion urbaine, animation sociale et urbaine) sont dispensées en IUT et en dépit de quelques conventions d'enseignement ici ou là entre quelques centres de formation et des départements d'universités (cursus de sociologie, administration économique et sociale, sciences de l'éducation...), alors que la situation est bien différente chez nos voisins européens (filières universitaires de travail social, pédagogie sociale, orthopédagogie...) ou d'outre-Atlantique (travail social, psycho-éducation...);

- une réforme touchant l'ensemble des diplômes et marquée par l'apparition de référentiels (métier, de compétences, de formation...), désormais indispensables pour les procédures de certification par validation des acquis de l'expérience (VAE)... et pour la formation pratique par la disparition des «formateurs terrain» (modèle du compagnonnage) et l'émergence de «sites qualifiants».

C'est certainement l'assimilation de l'action des professionnels du travail social à une «prestation de service» et l'introduction de référentiels professionnels, à partir desquels sont élaborés référentiels de certification et référentiels de formation, qui ont contribué à une grande déstabilisation de l'univers de la formation professionnelle. Après la loi 2002-02 rénovant l'action sociale et médico-sociale avec la mise en place de référentiels et schémas, de procédures d'évaluation interne et externe destinées à promouvoir de «bonnes pratiques», c'est le domaine de la formation qui se trouve contraint d'adopter des procédures relevant d'un registre managérial peu compatibles avec la culture professionnelle et ses modes de régulation en vigueur, comme l'indique de façon très critique $\mathrm{M}$. Chauvière ${ }^{9}$. T. Piot ${ }^{10}$ en vient à opposer deux modèles concurrents de professionnalisation des formateurs du secteur social: un «modèle managérial centré sur la maîtrise des savoirs techniques au niveau de l'organisation, du droit de la gestion » et un «modèle "réflexif-critique" centré sur une culture de la relation de formation et intégrant des savoirs techniques et organisationnels».

\section{La formation professionnelle continue}

Quant à la formation professionnelle continue, elle est dispensée par les centres de formation impliqués dans la formation professionnelle initiale ou par des organismes limitant leurs activités à la formation et à la consultation. La pluralité des conditions d'exercice professionnel des intervenants sociaux renforce la nécessité des remises

\footnotetext{
9. Chauvière 2007.

10. Piot 2007, 100.
} 
à jour et des activités de perfectionnement, d'où un développement important de la formation continue, notamment des pratiques de supervision d'équipes et de groupes d'analyse de la pratique ${ }^{11}$. De plus en plus, la part des formations «intra» ou «sur site » prend le dessus sur les formations «inter» jugées trop onéreuses et peu efficaces.

Les formations supérieures principalement destinées à l'encadrement ont été profondément rénovées ces dernières années:

- apparition en 2004 du Caferuis (Certificat d'aptitude aux fonctions d'encadrement et de responsable d'unité d'intervention sociale), diplôme homologué de niveau II, initialement institué pour l'encadrement des métiers de l'aide à domicile et qui semble répondre à des attentes plus importantes du secteur social pour la formation des cadres de proximité;

- disparition du DSTS (Diplôme supérieur de travail social), peu reconnu statutairement par les employeurs, et instauration en 2006 du DEIS (Diplôme d'État d'ingénierie sociale), désormais diplôme homologué de niveau I;

- réforme en 2002 du CAFDES (Certificat d'aptitude aux fonctions de directeur d'établissement social), diplôme homologué de niveau I et comportant quatre options: enfance inadaptée, personnes adultes handicapées, personnes en difficulté sociale, personnes âgées. Sachant par ailleurs que des professionnels issus de l'enseignement spécialisé dirigent de nombreux établissements médico et / ou socio-éducatifs après obtention du DDEEAS (Diplôme de directeur d'établissement d'éducation adaptée et spécialisée) préparé à l'INSHEA (Institut national supérieur de formation et de recherche pour l'éducation des jeunes handicapés et les enseignements adaptés) à Suresnes.

C'est d'ailleurs davantage en direction de l'encadrement que l'offre de formation universitaire s'est développée.

\section{Le rapport à l'Université et la place des sciences de l'éducation}

Contrairement à d'autres pays, l'appareil de formation professionnelle initiale des travailleurs sociaux s'est édifié en France hors de l'Université. Néanmoins, outre les quelques formations dispensées en IUT déjà signalées, on relève un partenariat, sans doute assez limité, entre certaines filières (sociologie, administration économique et sociale, sciences de l'éducation...), pour la préparation de diplômes homologués de niveau III. L'obtention de tels diplômes permet aux professionnels d'accéder à l'Université (licence). Ils sont d'ailleurs assez nombreux à s'y inscrire et à poursuivre des études universitaires au-delà de la licence depuis les années 1980, notamment en $3^{\text {e }}$ cycle, DESS et DEA, et aujourd'hui en master.

Les travailleurs sociaux désireux de s'inscrire à l'Université semblent davantage s'orienter vers la sociologie et les sciences de l'éducation, dans la mesure où la

11. Ion \& Ravon 2002; Fablet 2008. 
discipline psychologie dispose de sa propre filière de formation professionnelle: les psychologues (titre reconnu depuis 1985), qu'elle protège en restreignant l'offre de formation aux professionnels extérieurs. On impose en effet aux titulaires de diplômes de travailleurs sociaux de niveau III désireux de poursuivre des études de psychologie d'effectuer un parcours presque complet (accès possible seulement en $1^{\mathrm{re}}$ ou $2^{\mathrm{e}}$ année de licence), ce qui se révèle dissuasif en dépit de l'attractivité de la profession de "psy» pour un nombre important de travailleurs sociaux (la référence pour les éducateurs se révélant être bien souvent la relation psychothérapeutique). Ne formant pas de professionnels reconnus par un titre spécifique, à l'instar des psychologues, mais à des métiers et / ou fonctions (formateurs, consultants, études, recherches, et autres formes d'expertise...), la sociologie et les sciences de l'éducation se montrent des disciplines en général plus accueillantes (accès possible en $3^{\mathrm{e}}$ année de licence, avec parfois des allégements de formation tenant compte des acquis de l'expérience professionnelle).

C'est surtout au niveau de l'encadrement que l'offre de formation universitaire est apparue progressivement menaçante pour les centres de formation du secteur professionnel. Si les diplômes d'université (DU) ainsi que les diplômes des hautes études en pratiques sociales (DHEPS) proposés par les collèges coopératifs initiés par $\mathrm{H}$. Desroches ont eu un certain succès et permis le développement de la professionnalisation durant la période 1975-2000, c'est davantage vers des certifications mieux établies que les travailleurs sociaux se sont depuis tournés. Le rôle joué par le réseau universitaire des formations du social (RUFS) apparaît ici non négligeable. Animé par des universitaires soucieux de proposer un accès à l'Université aux professionnels du secteur social, le plus souvent via des services de formation continue, le RUFS ${ }^{12}$ a œuvré, notamment au niveau du DSTS, à un rapprochement entre universités et centres de formation professionnelle, après la réforme de ce diplôme en 1998 qui rendait obligatoire ce type de partenariat.

La réforme LMD (licence / master / doctorat) a contribué à accentuer la concurrence entre les différentes catégories d'acteurs du fait de la démultiplication de l'offre de formation, notamment par la création de masters professionnels selon trois orientations: politiques sociales (insertion sociale, familles...), management du social et du médico-social, formation de formateurs d'intervenants sociaux, destinés à former des personnels d'encadrement et de formation du secteur social.

Peu de temps auparavant, la création en 2000 d'une chaire de travail social au CNAM (Conservatoire national des arts et métiers) constitue un signe timide de reconnaissance pour la recherche prenant pour objet le travail social et l'intervention sociale. Convient-il de considérer qu'il s'agit là des prémices de l'édification d'une nouvelle discipline universitaire? Même si les milieux professionnels revendiquent depuis longtemps la création d'une discipline du travail social, il est probable qu'elle ne voie pas le jour de sitôt. En effet, la recherche scientifique prenant pour objet le social se révèle

12. De Montalembert 2006; Sanchou 2007. 
très dispersée entre les différentes disciplines en sciences humaines, même si la sociologie apparaît hégémonique en ce domaine. La chaire de travail social relève d'ailleurs de cette discipline et, au sein du RUFS, c'est la sociologie qui est la mieux représentée; aussi, à l'instar des cursus d'administration économique et sociale, c'est plutôt la constitution d'une filière de formation universitaire à l'enseigne de l'intervention sociale qui serait souhaitée, à défaut de promouvoir une nouvelle discipline.

Qu'en est-il de l'apport spécifique de la discipline sciences de l'éducation, notamment depuis la dernière édition en 2001 de la «brochure bleue» de l'AECSE (Association des enseignants et chercheurs en sciences de l'éducation)? Même si le paysage institutionnel du secteur social s'est profondément transformé au cours de la dernière décennie, la place occupée par la discipline n’a guère évolué.

Dans le domaine de la formation des professionnels de l'intervention sociale, le partenariat des départements de sciences de l'éducation avec des centres de formation apparaît relativement limité au regard du nombre de conventions d'enseignement signées, tout comme l'est l'offre spécifique de formation destinée à cette catégorie d'étudiants, même s'ils sont assez nombreux à s'inscrire et à poursuivre des études de sciences de l'éducation.

Du point de vue de la recherche, qu'il s'agisse des thèses soutenues ou de l'implantation dans le secteur de la recherche contractuelle (MIRE, CNAF...), l'apport des sciences de l'éducation se révèle bien moins important que celui de la sociologie, discipline à n'en pas douter prédominante dans le champ de l'intervention sociale depuis plus d'une vingtaine d'années ${ }^{13}$ et parfois inquiète des tendances à «la psychologisation» de l'intervention sociale ${ }^{14}$.

Davantage centrée sur l'école, l'enseignement et la formation des adultes, la discipline des sciences de l'éducation s'est tardivement intéressée à l'éducation familiale et aux interventions socio-éducatives. Entendue à la fois comme un champ de pratiques éducatives spécifique et de recherches sur ces pratiques, l'éducation familiale s'intéresse aux activités éducatives intrafamiliales, c'est-à-dire principalement des parents à l'égard des enfants, mais également aux interventions sociales mises en œuvre par une assez grande diversité de professionnels pour former, soutenir, aider, voire suppléer les parents; soit le domaine des interventions socio-éducatives ${ }^{15}$.

La seule équipe de recherche à cette enseigne, créée par P. Durning (1995 / 2006) qui dirige depuis 2004 l'Observatoire national de l'enfance en danger (ONED), est une composante du Centre de recherche éducation et formation (CREF EA 1589) de l'université de Nanterre. Insérée dans plusieurs réseaux - le réseau REEFI (l'éducation des enfants: entre familles et autres institutions éducatives), l'AIFREF (Association internationale de formation et de recherche en éducation familiale) et l'EUSARF (European Scientific Association for Residential and Forster Care for Children and

\footnotetext{
13. Bailleau et al. 1985 .

14. Bresson 2006.

15. Fablet 2002 et 2007 .
} 
Adolescents) - cette équipe assure depuis 1997 la publication de La revue internationale de l'éducation familiale. Signalons non loin de Nanterre, à Suresnes, l'émergence de l'INSHEA qui s'est substitué le $1^{\text {er }}$ janvier 2006 au CNEFEI (Centre national d'études et de formation pour l'enfance inadaptée) et qui est appelé à développer recherches et formations dans le domaine de l'éducation et des enseignements adaptés et spécialisés. L'INSHEA publie La nouvelle revue de l'adaptation et de la scolarisation, qui a pris la suite en 2006 de La nouvelle revue de l'AIS.

Les activités de recherche en sciences de l'éducation relatives au secteur social apparaissent donc assez dispersées au sein des différents pôles universitaires (Lille, Lyon, Paris, Toulouse...) et témoignent davantage d'initiatives individuelles d'enseignantschercheurs, du fait sans doute d'une insuffisante structuration de la recherche de la discipline en direction de ce secteur. Ce n'est pourtant pas là le signe d'un désintérêt puisque, au cours de l'année 2007, plusieurs revues relevant de la discipline - Questions vives (Université de Provence), Les sciences de l'éducation. Pour l'ère nouvelle (Université de Caen), Les dossiers des sciences de l'éducation (Université de Toulouse) - ont publié des numéros spéciaux consacrés au travail social, sachant que la revue Recherche et formation $\mathrm{n}^{\circ} 54$ (INRP) de la même année traite de l'offre de formation universitaire de la discipline quant à la formation professionnelle des formateurs.

On concevra donc aisément que dans une période qui a vu décroître, parfois de façon alarmante, le nombre d'inscriptions dans divers cursus de sciences humaines, la discipline des sciences de l'éducation aurait tout intérêt, tant en matière de recherche que de formation, à accentuer ses efforts pour ouvrir davantage de perspectives aux professionnels de l'intervention sociale.

Dominique FABLET ${ }^{16}$

CREF-EA

Université de Nanterre - Paris $X$

\section{Références bibliographiques}

Aecse (2001), Les sciences de l'éducation. Enjeux, finalités et défis, Paris, AECSE - INRP.

Bailleau F., Lefaucheur N., Peyre V. (dir.) (1985), Lectures sociologiques du travail social, Paris, Les éditions ouvrières.

Beynier D., Tudoux B., Momic M. (2005), « Les métiers du travail social hors aide à domicile», Études et résultats, $n^{\circ} 441$.

16. Équipe de recherche "Éducation familiale et interventions sociales auprès des familles", Centre de recherche éducation et formation (CREF-EA 1589), Université Paris Ouest - Nanterre - La Défense. 
BREssé S. (2004), «Le personnel des services d'aide à domicile en 1999», Études et résultats, n 297.

BRESSON M. (dir.) (2006), La psychologisation de l'intervention sociale: mythes et réalités, Paris, L'Harmattan.

Chauvière M. (2007), Trop de gestion tue le social. Essai sur une discrète chalandisation, Paris, La Découverte.

Chauvière M., Tronche D. (dir.) (2002), Qualifier le travail social. Dynamique professionnelle et qualité de service, Paris, Dunod.

Chauvière M., Ladsous J., Belorgey J.-M. (dir.) (2006), Reconstruire l'action sociale, Paris, Dunod.

Chopart J.-N. (dir.) (200o), Les mutations du travail social. Dynamiques d'un champ professionnel, Paris, Dunod.

De Montalembert M. (2006), «Une reconfiguration (en cours) de la formation. Entre école et Université», Informations sociales, n 135, p. 72-80.

Fablet D. (coord.) (2002), Les interventions socio-éducatives. Actualité de la recherche, Paris, L'Harmattan.

FABLET D. (coord.) (2007), Les professionnels de l'intervention socio-éducative. Modèles de référence et analyses de pratiques, Paris, L'Harmattan.

FABLET D. (coord.) (2008), Intervenants sociaux et analyse des pratiques, Paris, L'Harmattan.

Grenat P. (2006), «Les étudiants et les diplômés des formations aux professions sociales de 1985 à 2004", Études et résultats, n’ 513.

IgAs (2006), L'intervention sociale, un travail de proximité. Rapport annuel 2005, Paris, La Documentation française.

IoN J. (1998), Le travail social au singulier, Paris, Dunod.

Ion J., Ravon B. (2002), Les travailleurs sociaux, Paris, La Découverte.

Laville J.-L. (2005), Sociologie des services. Entre marché et solidarité, Ramonville-SaintAgne, Érès.

Ріот T. (2007), «Formation de formateurs dans le travail social. Un espace de tensions plurielles", Recherche et formations, $\mathrm{n}^{\circ}$ 54, p. 87-100.

SANCHOU P. (2007), "Les travailleurs sociaux et leurs formations: quelques enjeux actuels", Les dossiers des sciences de l'éducation, $\mathrm{n}^{\circ} 17, \mathrm{p} .13-23$.

Woitrain E. (2000), «Les travailleurs sociaux en 1998: environ 800 ooo professionnels reconnus», Études et résultats, n 79. 



\section{LES RECHERCHES EN SCIENCES DE L'ÉDUCATION ET DANS LES DISCIPLINES ÉPONYMES}





\section{PRÉSENTATION : MATURITÉ ET INDÉPENDANCE}

Sociologie, histoire, psychologie, philosophie... définissent des questions théoriques et des domaines de recherche anciens, au passé souvent prestigieux, auprès desquels les sciences de l'éducation peuvent apparaître bien modestes. Mais on pourra remarquer que la psychologie, quand elle s'est constituée comme science à la fin du XIX ${ }^{e}$ siècle, s'est directement intéressée, avec Binet et Claparède, à des questions d'éducation, et que la philosophie, ancêtre tutélaire, s'est dès son origine penchée sur le problème de la genèse des connaissances, sur les conditions de la pédagogie et sur le sens de l'éducation dans la politique de la cité. Mais il est vrai également que les sciences de l'éducation, dans leurs spécialisations régionales, se sont constituées à partir de ces disciplines mères: elles en ont défini les soubassements théoriques et méthodologiques.

Ainsi la question posée par le colloque est double: les développements de la sociologie, de l'histoire, de la psychologie et de la philosophie dans les espaces nouveaux ouverts par les sciences de l'éducation les ont-elles amenées à définir de nouveaux objets, poser des questions inédites, spécifier leurs méthodes? Corollairement, la sociologie "de l'éducation", la psychologie "de l'éducation", l'histoire «de l'éducation» et la philosophie «de l'éducation» ont-elles conquis, au cours de leur courte histoire, leur autonomie vis-à-vis des disciplines éponymes, en termes de problématiques, d'élaborations théoriques et d'approches méthodologiques?

Les contributions présentes dans cette troisième partie de l'ouvrage vont tour à tour aborder ces questions et fournir des réponses qui nous amèneraient à conclure qu'au cours de ces quarante années, les sciences de l'éducation, avec une diversité qui fait leur richesse, ont acquis une solidité et une pertinence qui devraient convaincre les plus sceptiques.

Yves Dutercq montre comment la sociologie française, de Durkheim à Bourdieu, s'est abondamment préoccupée des problèmes d'éducation et de socialisation, tandis qu'à partir des années soixante, les questions d'éducation ont largement puisé dans les travaux des sociologues. Cette convergence n'a fait que se renforcer, mais s'est aussi démultipliée: la recherche en éducation s'est diversifiée et la sociologie ne saurait faire cavalier seul; à côtoyer ses disciplines sœurs en sciences de l'éducation, la recherche en sociologie de l'éducation a progressivement construit des problématiques spécifiques et renouvelé, de façon relativement autonome par rapport à la discipline mère, ses questions et ses approches; il analyse en particulier 
en quoi, par quels biais, l'articulation entre sociologie et autres sciences de l'éducation a de nombreuses incidences sur la démarche du sociologue en éducation: c'est dans ces va-et-vient, ces frottements, voire les débats que les confrontations suscitent, que les sciences de l'éducation "apportent» quelque chose de neuf à la sociologie, en même temps que cette dernière les incite à «sortir» de leurs cadres familiers. À partir de l'ethnologie, les analyses de Julie Delalande aboutissent à des conclusions similaires et vont plus loin : elle montre qu'à la convergence entre la tradition anthropologique et les méthodes de l'investigation ethnologique, de nouvelles approches se sont mises en place concernant l'étude de l'enfance et qu'une nouvelle discipline en particulier est en train de naître sur les frontières, dans les croisements ou les juxtapositions: l'ethnosociologie de l'enfance, domaine pluridisciplinaire s'il en est.

Concernant les recherches historiques, François Jacquet-Francillon indique que l'histoire de l'éducation et de l'enseignement est une histoire-mémoire qui présente trois caractéristiques principales: i) elle se centre sur des auteurs et des œuvres; ii) elle s'intéresse aux origines et au devenir des doctrines et des institutions; iii) elle prête aux idées dans l'histoire une efficacité particulière. Ses avancées au cours des dernières années méritent d'être remarquées, en particulier sur le terrain de l'histoire des savoirs scolaires - où elle manifeste sa différence et son originalité par rapport aux cadres canoniques de l'histoire socioculturelle et de l'histoire patrimoniale.

À partir de cela, Jean-Noël Luc peut analyser l'essor d'un champ de recherche, qui s'est développé à partir des années soixante-dix, à l'intérieur de la discipline historique mais en travaillant aussi sur ses frontières et avec d'autres disciplines : la sociologie chez Viviane Isambert-Jamati, la philosophie avec Mona Ozouf...; mouvement qui s'accompagnait d'un important développement éditorial: création de revues ou de collections. De nouveaux territoires et / ou de nouveaux objets d'études sont apparus, mobilisant des dynamiques plus larges : économiques, démographiques, sociales ou religieuses. Dans ce contexte, «l'historien de l'éducation est amené à traverser avec profit d'autres champs de recherche, relatifs à l'histoire de la famille, des enfants, des jeunes et du genre». Ainsi, riche de sa pluralité et de la transversalité de ses curiosités, l'histoire de l'éducation peut elle accéder à la place privilégiée d' «observatoire d'une société».

Quant à Loïc Chalmel, il développe plus particulièrement dans sa communication l'idée que la recherche historique en éducation travaille sur la mémoire et sur l'archive - entre les traces de pratiques devenus lointaines et le sens que des sujets leur ont accordées. Du point de vue épistémologique, il défend la thèse d'une histoire herméneutique de la pédagogie (des idées ou des doctrines pédagogiques).

Jusqu'à une période récente, la philosophie de l'éducation a connu un statut et une identité problématique. Quand est-elle devenue un domaine «détachable» de la philosophie pour définir ses objets et ses méthodes de façon propre? Dans une première période, elle est identifiée parmi les sciences de l'éducation à partir des tâches qui lui sont dévolues: la réflexion sur les valeurs et les fins de l'éducation. Il 
a fallu attendre les années $1980^{1}$; pour qu'elle trouve son identité théorique et institutionnelle (dans les départements de sciences de l'éducation à l'Université, et pour la formation des enseignants dans les Écoles normales et les IUFM). Les rencontres, les confrontations, les interrogations sur l'identité de la discipline ont conduit au cours de cette période la communauté des chercheurs à définir ses domaines de réflexion à partir de trois grands axes : l'orientation axiologique (où elle croise la philosophie morale et la philosophie politique), l'élucidation critique (herméneutique) des réalités éducatives : pratiques, discours pédagogiques, dispositifs institutionnels et didactiques..., l'analyse épistémologique enfin (où elle croise l'épistémologie des sciences) qui peut porter tour à tour sur la genèse et les conditions de constitution des discours de la formation, des théorisations pédagogiques, sur la généalogie des problématiques de l'éducation ${ }^{2}$. Dans sa communication, Jean Houssaye reprend la question de la constitution de la philosophie de l'éducation en se penchant sur l'histoire des professeurs de philosophie dans les Écoles normales à partir des missions qui leur furent confiées par l'Inspection générale dans la formation des maîtres : c'est dans ce cadre institutionnel, à la fois contraint et ouvert, que cette communauté professionnelle a défini, inventé, exploré des questions spécifiques - délaissées par la philosophie universitaire, leur a donné corps à partir d'hypothèses théoriques extrêmement diverses pour délimiter in fine un domaine propre, animé par les tensions et les dynamiques qui ont présidé à son émergence et qui entretiennent sa vitalité. De ce travail, Didier Moreau fournit une belle illustration, en se livrant à la déconstruction critique des discours politiques sur l'école à partir des travaux de Derrida qui fait de l'éducation une question centrale en philosophie et de l'herméneutique philosophique fondée par Gadamer autour de la question de la Bildung.

Pour présenter la place qui revient, dans cet éventail, à la psychologie de l'éducation, nous céderons la parole à Jacques Arveiller, qui s'était livré avec brio à cette tâche le deuxième jour du colloque:

Psychologie et sciences de l'éducation sont des disciplines sœurs. Au moment de leur fondation universitaire, en 1967, les sciences de l'éducation se situent dans la filiation de cette autre discipline, qui est son aînée de vingt ans. Mais la psychologie, quoi qu'en ait voulu Daniel Lagache en 1947, n'est pas unifiée, ni dans ses modèles, ni dans ses méthodes, ni dans ses pratiques. On pouvait penser, en 1967, qu'un tel projet d'unification autour du paradigme psychanalytique était encore en marche. Les quarante ans qui ont suivi ont prouvé qu'il n'en était rien, et la psychologie a persisté dans ses branches spécialisées, entre autres et pour ce qui nous occupe aujourd'hui : psychologie sociale, psychologie du développement, psychologie clinique et pathologique, sans oublier son dernier et vigoureux rejeton, les sciences cognitives.

1. Drouin-Hans \& Hannoun 1994.

2. Vergnioux 2006. 
Mais ce même Lagache avait introduit en 1947 dans notre pays une autre nouveauté de taille: le fait que la psychologie universitaire puisse déboucher, pour ses étudiants, sur des pratiques socialement opératoires à large échelle, visant certes à étudier la réalité des hommes, mais aussi à transformer cette réalité. Projet réalisé, celui-là, d'une psychologie praticienne, et plus seulement d'une pratique tout juste périphérique à celle du laboratoire, comme du temps d'Alfred Binet.

Pas plus que la psychologie n'a trouvé d'unité, la part des sciences de l'éducation qui s'en inspire depuis quarante ans est partagée dans ses modèles. Les fondateurs, comme Gaston Mialaret ou Maurice Debesse restaient psychologues de l'enfant, avec une référence tant sociale (Henri Wallon) que génétique (Jean Piaget), et une ambition expérimentale. La génération qui a suivi a témoigné d'avancées importantes du côté clinique et psychodynamique, à partir du modèle freudien, pouvant donner l'impression, pendant une vingtaine d'années, d'une référence massivement binaire, entre des anciens, tenants d'une psychologie restée traditionnelle, et des nouveaux centrés sur la psychanalyse. Mais les directions développées ultérieurement ont connu d'autres inspirations, la psychologie cognitive a connu son essor, et s'il persiste aujourd'hui bien des manières de faire de la psychologie en général, les manières de s'y référer ne sont pas moins nombreuses dans l'espace des sciences de l'éducation.

Dès lors qu'elles ont pris consistance, institutionnellement parlant, les sciences de l'éducation ont pu reprendre à leur compte, élargir et développer, cette branche définie dès 1904 par Édouard Claparède, d'une pédagogie basée sur la connaissance psychologique de l'enfant, d'une psychopédagogie. Avec ce risque d'un côté de ne voir dans l'exercice pédagogique que terrain d'application de connaissances produites ailleurs, sur le modèle sciences fondamentales $v s$ sciences appliquées. Avec certes la garantie corollaire d'une frontière toujours poreuse, jamais fermée, entre les développements désormais parallèles de la psychologie d'une part et des sciences de l'éducation de l'autre. Mais avec ce nouvel écueil, émergent, d'un recouvrement de travaux finalement comparables, menés de part et d'autre de la frontière, de nouvelles questions. En psychologie de l'enfant, comment en effet ne pas intégrer la dimension des apprentissages? Et si l'on se penche sur la pédagogie, sauraiton, de l'autre côté, ne pas tenir compte de celle qui s'adresse aux enfants?

Entre les sœurs parfois ennemies, il y a d'autres inconforts: risquer de voir, pour un pédagogue, les règles d'une bonne pédagogie définies par d'autres disciplines que la sienne; ou encore les soupçons, voire les procès dénigrant la valeur scientifique d'une recherche qui, comme la recherche en éducation, resterait fondamentalement au service d'une visée de modification, voire d'amélioration des pratiques pédagogiques. Mais la psychologie elle-même, avec d'autres sciences humaines, échapperait-elle donc à ce travers? Serait-elle donc une discipline si bien fondée épistémologiquement que cela justifierait un établissement institutionnel indiscutable? De grâce, ma sœur, un peu de modestie et de parité dans la fratrie, je vous prie. La cadette possède aussi sa personnalité et ses droits. Et d'ailleurs: si l'on remonte un peu plus avant, qui est réellement la cadette de l'autre, de la pédagogie et de la psychologie?

Rassurons-nous cependant: pour notre discipline, l'âge de la maturité, s'il existe, n'est pas celui de la non-contradiction, qui est l'apanage de la sénescence et 
de la mort. Allons plus loin : les sciences de l'éducation peuvent sans doute prétendre à une telle maturité, non pas en dépit, mais peut-être grâce aux relations polymorphes, contradictoires, parfois conflictuelles, qu'elles ont toujours entretenues et qu'elles continuent à entretenir avec les sciences psychologiques. Tant mieux. La conférence de Claudine Blanchard-Laville va, je le pense, illustrer cette vitalité, du côté clinique et psychanalytique.

\author{
Alain VergNiouX \\ CERSE \\ Université de Caen Basse-Normandie
}

\title{
Références bibliographiques
}

Drouin-Hans A.-M., Hannoun H. (dir.) (1994), Pour une philosophie de l'éducation, Dijon, CNDP-CRDP.

Vergnioux A. (2006), "Philosophie et éducation», in Traité des sciences et des pratiques de l'éducation, J. Beillerot, N. Mosconi (dir.), Paris, Dunod, p. 489-50o. 



\section{LES RECHERCHES EN SCIENCES DE L'ÉDUCATION ET DANS LES DISCIPLINES ÉPONYMES}

- La sociologie - 



\title{
SOCIOLOGIE, SOCIOLOGIE DE L'ÉDUCATION ET SCIENCES DE L'ÉDUCATION
}

\begin{abstract}
Résumé: Il ne semble pas que le sociologue ès qualité ait des atouts particuliers par rapport à son collègue sociologue de l'éducation quand il prend pour objet une question d'éducation, sinon une légitimité et une autorité institutionnelle plus élevées. À l'inverse, le sociologue de l'éducation n'a guère d'avantages hormis peut-être la nécessité de recourir à d'autres points de vue que ceux de la sociologie pour mener à bien ses recherches. À cet égard, la dynamique du travail collaboratif en sciences de l'éducation permet de consolider la pertinence des questions, de capitaliser les expertises et de favoriser la réflexion critique en même temps que la prudence des interprétations.
\end{abstract}

Mots clés: changement, objet de recherche, sciences de l'éducation, sociologie, sociologie de l'éducation.

La sociologie occupe une place privilégiée dans la recherche en éducation pour au moins deux raisons: d'une part, nos grandes figures de la sociologie en France (et pas seulement), se sont préoccupées abondamment des problèmes d'éducation et de socialisation, Durkheim, pour ne citer que lui, ayant été à la fois professeur de sociologie et de science de l'éducation ${ }^{1}$; d'autre part, les sciences de l'éducation, quand elles se sont organisées en discipline académique, ont largement construit leur reconnaissance autour du travail sociologique (qu'on se rappelle le colloque d'Amiens de 1967, moment assez unique de réflexion plurielle entre chercheurs, enseignants, syndicalistes et politiques). Cette convergence pose encore question aujourd'hui: la recherche en éducation s'est diversifiée, la sociologie ne prétend plus occuper une place hégémonique au sein des sciences de l'éducation, les sociologues de l'éducation oscillent entre sociologie et sciences de l'éducation, etc.

Ainsi peut-on s'interroger: le travail et les manières de travailler du sociologue sont-ils particulièrement propices à l'élucidation des questions d'éducation? Y a-t-il une communauté identifiable de la recherche en sociologie de l'éducation, à la fois distincte des et articulée aux deux mondes de la sociologie et des sciences de l'éducation? Et, pour tout dire, fait-on de la recherche sociologique de la même

1. Gautherin 2002 . 
manière en sociologie et en sciences de l'éducation? Les réflexions qui suivent ne prétendent pas répondre à ces questions mais simplement poser quelques éléments de débat, sans tabou.

\section{Recherche sociologique et recherche en éducation}

Se demander si sociologie et sciences de l'éducation font appel à la même recherche sociologique, c'est poser implicitement une question qui a toujours pesé sur les sciences de l'éducation dans leur aspiration à être acceptées comme pleine discipline scientifique: $\mathrm{y}$ a-t-il une recherche en éducation qui ne relève pas, en fait, d'une discipline particulière, déjà constituée, classique (histoire, psychologie, sociologie, etc.)?

Effectivement, la sociologie peut être a priori considérée comme une discipline de recherche établie, avec un ensemble de concepts et de méthodes reconnus et des figures tutélaires, alors que l'éducation est d'abord un objet ou un terrain de recherche.

Il est possible encore de s'interroger sur ce problème en observant les chercheurs et leur domaine d'intervention: qui sont les sociologues de l'éducation? Constituent-ils un groupe identifié et cohérent? De ce point de vue, il faut sans doute faire la différence entre, d'une part, les sociologues de l'éducation inscrits dans le champ académique et institutionnel de la sociologie qui, la plupart du temps, travaillent ou ont travaillé sur d'autres objets que l'éducation (Durkheim s'est intéressé au suicide, aux mythes et aux religions, Bourdieu aux pratiques sociales, à l'art et aux médias, etc.) et d'autre part les sociologues de l'éducation travaillant en sciences de l'éducation, qui se consacrent par nécessité exclusivement à l'éducation et à ce qui lui est connexe, formation ou socialisation, sans pour autant pratiquer d'ouverture disciplinaire.

Or l'idée de nombreux fondateurs des sciences de l'éducation était, au contraire, que leur objet devait permettre de recomposer des démarches disciplinaires au départ différentes pour constituer un corps de connaissances spécifique et intégré, par exemple à partir de la psychologie et de la sociologie. Ce fut le cas de Jacques Ardoino, auquel s'opposa à l'époque Viviane Isambert-Jamati dans des débats qui ont marqué le milieu. Viviane Isambert-Jamati se préoccupa plutôt de faire reconnaître institutionnellement des sous-disciplines originales, au premier rang desquelles elle mettait bien évidemment la sociologie de l'éducation. Mais on peut considérer, sans lui faire injure, que ses efforts furent vains, puisque son laboratoire de l'Université Paris V perdit, après son départ, la reconnaissance du CNRS et se fit absorber par un gros centre de recherche en sociologie, dont l'éducation demeura un objet d'analyse... parmi d'autres.

Et c'est bien là un premier écueil ou une première différence importante à faire, qui tient à la moindre faculté d'ouverture des sociologues inscrits en sciences de l'éducation que de ceux qui sont en sociologie et qui ont une large possibilité de diversifier leurs objets. Ce serait ainsi, contrairement à ce qu'on aurait pu imaginer d'emblée, les chercheurs en éducation qui seraient contraints de se montrer moins ouverts ou moins pluriels que les sociologues! 
Si l'on pose le problème en termes institutionnels, il faut rappeler qu'il existe en France deux sections universitaires distinctes, la $19^{\mathrm{e}}$ pour la sociologie, la $70^{\mathrm{e}}$ pour les sciences de l'éducation. Or si les sociologues de l'éducation de la $19^{\mathrm{e}}$ section se désignent d'abord comme sociologues et sont reconnus académiquement comme tels, ceux de la $70^{\mathrm{e}}$ sont, pour l'institution, des enseignants-chercheurs en sciences de l'éducation et, aux yeux de leurs collègues, considérés parfois comme des chercheurs en éducation, parfois comme des sociologues de l'éducation, plus rarement comme des sociologues.

Une autre manière de poser la question renvoie à un implicite qu'il ne serait pas honnête de cacher: celle de l'inégale dignité des sections universitaires de référence, qui peut avoir une incidence sur celle des recherches menées. Dans ce cas, la question posée deviendrait au bout du compte: fait-on de la «meilleure» recherche en sociologie de l'éducation, selon qu'on est reconnu comme sociologue ou comme chercheur en éducation? Question sans réponse unilatérale, on l'aura deviné, mais qu'on doit avoir à l'esprit dans la suite de la réflexion proposée.

Une fois ces préliminaires posés, une fois le terrain déblayé de quelques-uns de ses pièges, il paraît possible de débattre de la question initiale en se focalisant sur les points qui suivent:

- en quoi consiste le travail du sociologue et en quoi le regard qu'il porte a-t-il de la pertinence et de l'intérêt pour la recherche en éducation?;

- l'objet de recherche éducation ou plutôt les objets de recherche éducatifs confèrent-ils une spécificité à la sociologie de l'éducation?;

- malgré ce qui a été dit plus haut, l'articulation entre sociologie et autres sciences de l'éducation a-t-elle une incidence sur la démarche de sociologie en sciences de l'éducation?;

- le fait d'être confronté, dans l'enseignement comme dans la recherche en sciences de l'éducation, à des professionnels de l'éducation, a-t-il une incidence sur les pratiques de recherche, par exemple dans le rapport aux institutions ou encore dans la préoccupation pour l'intérêt social des recherches menées?

Je précise d'emblée que pour m'aider dans mes supputations, j'ai fait appel à quelquesuns de nos collègues sociologues de l'éducation parmi lesquels je dois une dette particulière à Anne Barrère, Jean-Louis Derouet, Marie Duru et Patrick Rayou.

\section{Le travail du sociologue}

Les sociologues ont une prétention plus ou moins avouée à contribuer au changement de l'organisation et du fonctionnement de la société, mais ce changement exige, aujourd'hui plus que jamais, une pleine participation des individus sociaux. La participation, pour qu'elle ait un sens et une efficacité, doit s'appuyer sur une claire connaissance de l'existant et des possibilités alternatives de le transformer. Cette manière de concevoir le changement n'est sans doute pas la plus spectaculaire, mais elle est la plus réaliste et la plus durable. 
Pour en être persuadé, il suffit de regarder les travaux menés depuis plus de quinze ans sur l'évolution du service public. On connaît les impulsions données en France par l'État au tournant des années 1990, à l'instigation du Commissariat général du plan, mais les études anthropologiques font ressortir que les changements y ont procédé la plupart du temps par petites touches, par micro-innovations, les unes émanant des seuls fonctionnaires, qui cherchent ainsi à répondre aux difficultés pratiques qu'ils rencontrent, les autres correspondant à une traduction par les agents d'incitations venues d'en haut ${ }^{2}$. Dans tous les cas, ces personnels de service public font preuve d'une capacité interprétative et inventive assez étonnante: cette capacité est l'œuvre d'individus qui ont appris à décrypter le monde et à faire une lecture sociologique et psychologique des relations entretenues avec les autres, en particulier avec les usagers.

Décrire les difficultés des gens, décrire le travail de ceux qui cherchent à répondre à leurs difficultés, analyser les chaînes de connexion verticales (au premier rang desquelles figurent les incitations hiérarchiques) et horizontales (comme l'exemple des autres, les transports et échanges d'un univers à l'autre), mettre ainsi en évidence les rapprochements possibles, proposer un cadre interprétatif qui peut par la suite aussi permettre de construire un cadre d'action, voilà bien ce qui peut être du travail de sociologue.

L'ambition de cette sociologie-là est donc non seulement de faire le lien entre les actions individuelles et des schèmes explicatifs, mais aussi, en décrivant sur l'exemple, à partir de l'observation et de l'analyse des pratiques, de mettre en évidence, par comparaison, par regroupement, par recoupement, des catégories descriptives qui sont en même temps des catégories possibles d'interprétation.

En ce sens, le sociologue se livre au même travail que tout être social, qui passe son temps à vivre et interpréter des situations, et par là à construire et à activer des catégories interprétatives. La principale différence est que le sociologue en a fait son métier, il dispose d'une grande panoplie de références et peut sans doute plus rapidement que d'autres faire les rapprochements et les connexions nécessaires qui permettent de généraliser. Mais son travail est d'abord modeste: la plus grande qualité du sociologue me semble être justement de pouvoir passer de la simple position d'interactant, dans les situations d'enquête de terrain, à celle d'analyste, voire de conceptualisateur, position qui dans certains cas peut être de pure récupération... mais il y a beaucoup de mérite et d'intelligence à savoir raconter des histoires vraies, d'une manière qui «donne à réfléchir» ou «fasse prendre conscience».

Si la sociologie ne peut avoir la prétention de «faire changer», elle peut assurément contribuer ou parfois participer au changement. Elle a pour cela deux possibilités. La première consiste à décrire des situations inacceptables, notamment de domination ou de soumission, plus généralement d'injustice, et en cela susciter le changement - l'objectif que Bourdieu affirmait avoir donné à ses écrits sur la reproduction à l'école, c'était d'aider les enseignants à en prendre conscience. La seconde

2. Camus et al. 1993. 
possibilité de contribuer au changement est de décrire celui qui est en train de se faire, à travers l'étude de mobilisations, de processus, de dispositifs...

C'est dire que démarches et objectifs de la sociologie trouvent toute leur pertinence dans l'analyse de l'école et, plus globalement, de l'éducation : faut-il en conclure qu'il n'y a pas de spécificité de la sociologie de l'éducation?

\section{Y a-t-il une spécificité de la sociologie de l'éducation?}

Considérer que la démarche sociologique est particulièrement adaptée à l'investigation des problèmes d'éducation conduit à se demander si, à rebours, le fait de travailler sur un ensemble d'objets eux-mêmes particuliers et qui ont trait à l'éducation produit une spécificité, celle de la sociologie de l'éducation.

Julie Delalande (CERSE, université de Caen) privilégie le terrain de l'enfance et de ses modes de socialisation: si elle revendique que ce terrain et ces objets de recherche confèrent une spécificité à sa sociologie, elle se trouvera sans doute rapidement confrontée à la difficulté de différencier une sociologie de l'éducation qui s'intéresse aux enfants d'une sociologie-anthropologie plus globale de l'enfance, dont elle se réclame d'ailleurs également ${ }^{3}$. De la même manière, je travaille beaucoup sur les politiques d'éducation, ce qui me conduit non seulement à échanger constamment avec les spécialistes des politiques publiques ou avec les sociologues de l'action publique, mais encore à insister sur le fait qu'il n'y a pas de spécificité du domaine de l'éducation en la matière et que les outils et concepts qu'ils utilisent sont parfaitement opératoires pour le travailler. Anne Van Haecht n'a-t-elle pas écrit qu'au bout du compte les politiques d'éducation pourraient bien être des figures exemplaires des politiques publiques ${ }^{4}$ ?

Ces deux exemples tendraient à montrer que des objets aussi dissemblables que les politiques d'éducation et la socialisation scolaire des enfants ne sont pas traités de façon fondamentalement différente par les sociologues spécialisés dans l'éducation. Bien plus, ces sociologues vont chercher ailleurs, soit dans les domaines plus généraux de la sociologie politique ou de la sociologie de l'enfance, concepts, hypothèses explicatives et démarches de recherche. Le reconnaitre signifierait que la principale spécificité de la sociologie de l'éducation tiendrait à ses objets en tant que tels. On est sociologue de l'éducation parce qu'on poursuit des recherches sociologiques qui portent sur l'éducation: les démarches qu'on requiert pour cela sont celles de la sociologie, certaines de ces démarches plus spécifiques sont cependant communes à d'autres sociologies qui s'intéressent à d'autres objets de recherche.

Cela vaut pour la sociologie de l'éducation en général, mais si on poursuit dans la même logique, on devra faire la différence entre une sociologie de l'éducation pratiquée par les sociologues de sociologie et une autre pratiquée par les sociologues des sciences de l'éducation. Pourquoi alors ne pas se demander si le fait d'aborder les

\footnotetext{
3. Delalande 2001.

4. Van Haecht 1998.
} 
objets éducatifs avec la double casquette de sociologue et de chercheur en éducation apporte un plus par rapport à ceux qui les abordent comme simples sociologues? Ce plus serait à aller chercher du côté de la plus grande aptitude des premiers à ouvrir leur dispositif de recherche à d'autres regards que de pure sociologie.

\section{Qu'apportent les autres sciences de l'éducation à la sociologie de l'éducation?}

Qui travaille comme sociologue affilié aux sciences de l'éducation a la chance de côtoyer au sein des laboratoires auxquels il appartient des spécialistes de nombreux domaines, classiques comme la philosophie ou la psychologie, moins classiques comme les didactiques ou l'ergonomie. Le melting-pot est fructueux car il fournit de nombreuses occasions de discussions stimulantes et encore la possibilité de concevoir des dispositifs de recherche complexes, alliant des savoirs et des savoir-faire non seulement différents mais aussi complémentaires.

C'est cette expérience dont témoigne Patrick Rayou (ESCOL, université Paris VIII) quand il évoque son travail avec Luc Ria, spécialiste d'ergonomie cognitive, sur l'entrée dans le métier des enseignants néo-titulaires. Dans ce cas, ce que des entretiens sociologiques individuels classiques peuvent aider à mettre en évidence se trouve fortement enrichi du recours à des techniques comme le filmage vidéo de séquences de cours, l'autoconfrontation des jeunes enseignants avec la séquence dont ils ont été les acteurs, le débat avec les collègues qui les ont observés, surtout quand il s'agit, comme ici, de jeunes professeurs d'éducation physique et sportive ${ }^{5}$. À la suite des entretiens préalables, les commentaires faits lors du visionnement des séquences filmées permettent des analyses plus précises et font notamment émerger difficultés et émotions vécues à tel ou tel moment. Patrick Rayou va même jusqu’à considérer que les analyses livrées ainsi deviennent mieux capables de discriminer ce qui relève de la généralité ou de la particularité et, par exemple, de la construction d'un style propre à l'intérieur d'un genre professionnel enseignant.

Le plus intéressant pour le sociologue, c'est que cette approche met à l'épreuve de l'interprétation des situations les explications obligées renvoyant à des déterminants sociaux. Et tout spécialement quand les situations à interpréter correspondent à des moments de risque vécus par l'enseignant.

Une expérience similaire avec une équipe composée de sociologues et de didacticiens m'a beaucoup appris, lorsqu'il a fallu imaginer un dispositif d'enquête pertinent pour mener une recherche portant sur la diversification pédagogique au collège. Nous voulions étudier comment les enseignants de collège confrontés à un public hétérogène, et pour une bonne part en difficulté, répondaient aux exigences des situations de cours. Par ailleurs, nous souhaitions mettre en œuvre un véritable travail de sociologie du curriculum. Le groupe des sociologues qui s'était lancé dans l'aventure

5. Gelin et al. 2007 . 
se sentait tout à fait capable de mener une enquête par entretiens auprès des enseignants et des élèves, qui devait permettre de confronter trois niveaux de curriculum: le programme officiel, son interprétation par les enseignants, sa compréhension par les élèves. Pour le premier, il suffisait de lire les instructions du Ministère, pour la seconde et la troisième, des entretiens avec enseignants et élèves devaient nous éclairer. Mais comment donner du corps à de tels entretiens, comment éviter de simplement enregistrer la reconstruction idéale des enseignants et comment appréhender ce que les élèves avaient ou n'avaient pas compris? Seuls des entretiens construits autour de l'observation attentive et avertie de cours pouvaient aider à sortir des généralités. Sur ce point, le travail des sociologues s'est considérablement enrichi de la coopération avec le groupe des didacticiens de différentes disciplines scolaires avec lesquels avaient été constitués des duos et avait été élaboré un dispositif d'enquête sophistiqué, constitué d'entretiens ex ante avec les enseignants, d'observations de situations de classe et d'entretiens ex post avec enseignants et élèves ${ }^{6}$. L'objectif en était de confronter les différentes interprétations du cours proposées par les différents protagonistes, y compris les deux chercheurs chargés de l'observation: nous avons ainsi pu récupérer un matériau d'une grande richesse et un croisement de points de vue qui interdisait les explications toutes faites. Un simple exemple en donnera une idée.

Un professeur de mathématiques expérimenté et considéré dans son établissement comme un leader pédagogique, s'étonnait, après le cours que nous avions observé, que les élèves n'aient pas accroché et se montrait très amer de l'échec de ce cours, pourtant bien préparé. Il avait commencé par des exercices destinés à rappeler aux élèves une notion vue l'année précédente et dont ils avaient besoin pour comprendre les nouveautés abordées durant la seconde partie du cours. Les élèves avaient dans l'ensemble réussi ces exercices mais n'avaient visiblement pas assimilé le cours qui avait suivi. S'exprimant au cours d'un entretien collectif, ils s'étonnaient quant à eux que leur professeur leur ait proposé un cours ardu, exigeant des connaissances préalables qu'ils ne maîtrisaient plus et regrettaient qu'il ne leur ait pas fait d'abord un rappel sur ce point. Ils n'avaient absolument pas perçu les exercices liminaux comme destinés à cet effet! Ils n'avaient pas fait le lien entre les deux parties du cours que nous avions observé: inattention de leur part? Insuffisante insistance de l'enseignant? Peu importe: du point de vue pédagogique, l'échec était patent. Seule l'observation armée, facilitée par le double regard du sociologue et du didacticien des mathématiques, avait permis d'aller suffisamment loin dans l'entretien pour mettre au jour ce conflit quasi cognitif et cet écart dans le curriculum réel. Mais c'est aussi la coopération avec l'enseignant lui-même, dans le cadre d'une enquête au dispositif lourd et avec le risque assumé d'ébranler ses certitudes, qui avait permis d'en arriver là. De plus, cette coopération volontaire et consciente avait servi les objectifs de recherche aussi bien que la demande de réflexivité et de régulation de l'enseignant dans le cadre d'un donnantdonnant qui constitue une règle et un sésame du travail de recherche en éducation.

6. Dutercq \& Derouet 2004. 


\section{Un travail collaboratif entre chercheurs et enseignants}

Dès le début des années 1970, les sociologues anglais de l'Institute of Education de Londres, qui avaient entrepris de nombreuses enquêtes de terrain, se sont interrogés sur leur relation aux enseignants des établissements dont ils étudiaient le travail. Ils les observaient dans les classes, dans les salles de professeurs, ils observaient les élèves dans la cour de récréation, ils multipliaient leur présence sur le terrain, sans témoigner en retour de leur dette envers leurs enquêtés. Après quelques débats, ils décidèrent de ne plus travailler désormais sur, mais avec les enseignants, et de leur proposer des comptes rendus de leurs enquêtes, outre que ces travaux de terrain servaient bien sûr de base à la formation qu'ils proposaient dans les instituts de formation récemment créés et plus encore à l'Open University. Puis, allant plus loin, ils se mirent à associer complètement ces enseignants à leurs recherches en leur proposant de participer pleinement aux enquêtes ${ }^{7}$. Encore mieux: Andrew Pollard ${ }^{8}$ fit de même avec de jeunes élèves du primaire qui devinrent ses premiers observateurs des formes de socialisation que leurs camarades entretenaient à l'école. Il y eut durant toutes ces années d'ardents débats méthodologiques entre ces tenants de la new sociology of education qui influencèrent bien évidemment ce qui se construisit à la suite en France, à l'INRP.

L'originalité du travail réalisé dans les années 1980 par les chercheurs de l'INRP fut sans doute de deux ordres: d'une part, favoriser la collaboration entre spécialistes de différents domaines, comme dans l'enquête précédemment décrite, menée conjointement par sociologues et didacticiens; d'autre part, et de façon plus constante, permettre à des enseignants du terrain de participer aux études lancées par les équipes de l'Institut, sous le statut d'enseignants associés aux recherches. Ce type d'association fut particulièrement requis par les sociologues. C'est ainsi que naquit le Groupe d'études sociologiques qui systématisa cette manière de faire, comme en témoigne l'article de Jean-Louis Derouet, au titre évocateur: «Des enseignants sociologues de leur établissement $"$ ${ }^{9}$.

C'est là qu'on perçoit le mieux la spécificité des sciences de l'éducation, en particulier dans leur versant sociologique: elles sont incontestablement influencées par la présence des praticiens, que ce soit à l'échelon de la recherche, comme nous venons de le voir, que ce soit à l'échelon de l'enseignement, à la mesure de la forte présence parmi les étudiants des enseignants actuels ou en puissance. Cette présence crée une double dépendance: dépendance à l'égard de l'utilité sociale, car ces praticiens attendent bien normalement que la sociologie de l'éducation apporte des réponses à leurs difficultés, dépendance à l'égard de la demande institutionnelle, car le travail des sociologues de l'éducation s'apparente d'une manière ou d'une autre à des formes d'expertise ou d'évaluation qui répondent explicitement ou implicitement à cette

7. Hammersley 1999.

8. Pollard 1982.

9. Derouet 1985 . 
demande. Et c'est bien là tout l'intérêt et tout le risque de cette sociologie de l'éducation que d'avoir la possibilité de peser sur l'action tout en gardant l'exigence d'une extériorité suffisante.

\section{Pluralité des approches et acuité de l'analyse}

Il me semble que de telles considérations ne tiennent finalement pas tellement à la sociologie de l'éducation en tant que telle, mais bien à son éventuelle inscription dans les sciences de l'éducation en général, qui conditionnerait une certaine manière d'aborder les objets éducatifs.

Les travaux récents menés sur la question des inégalités scolaires font ressortir la pertinence d'analyses qui requièrent différents cadres et points de vue - comme le font les sciences de l'éducation. C'est ainsi que la synthèse proposée par Marie Duru ${ }^{10}$ montre toutes les combinaisons possibles entre prise en compte d'approches déterministes ou individualistes, typiquement sociologiques, et attention à la façon dont les acteurs réagissent en situation aux données contextuelles, y compris de façon affective, ce qui appelle une démarche à la fois interactionniste et psychosociologique. C'est encore la pluralité des approches et l'acuité d'une analyse nourrie de connaissances économiques, pédagogiques et sociologiques qui ont permis à Nathalie Mons ${ }^{11}$ de faire valoir qu'il n'y a pas incompatibilité entre la recherche d'efficacité et la recherche de justice dans le système scolaire.

Je ne crois pas qu'on fait une sociologie différente selon qu'on est sociologue de sociologie ou des sciences de l'éducation, mais il est vrai en revanche que la recherche en éducation confronte le sociologue à des dilemmes spécifiques. Je crois encore que, pour résoudre ces dilemmes, la chance du chercheur en sciences de l'éducation, qu'il soit sociologue, psychologue ou philosophe, c'est de pouvoir s'appuyer quasi naturellement sur l'expertise de collègues maniant une autre épistémologie et recourant à une autre méthodologie que la sienne propre. Il gagne ainsi en prudence d'interprétation et il prend un peu plus garde aux préconisations qu'on ne manquera pas d'attendre de lui.

Yves Duterce

CREN

Université de Nantes

10. Duru-Bellat 2002.

11. Mons 2008. 


\section{Références bibliographiques}

Camus A., Corcuff P., Lafaye C. (1993), «Entre le local et le national: des cas d'innovation dans le service public», Revue française des affaires sociales, vol. 47, n 3, p. 17-47.

Delalande J. (2001), La cour de récréation. Contribution à une anthropologie de l'enfance, Rennes, Presses universitaires de Rennes.

Derouet J.-L. (1985), «Des enseignants sociologues de leur établissement», Revue française de pédagogie, $\mathrm{n}^{\circ}$ 72, p. 113-124.

Duru-Bellat M. (2002), Les inégalités sociales à l'école, Paris, PUF.

Duterce Y., Derouet J.-L. (dir.) (2004), Le collège en chantier, Paris, INRP.

GaUtherin C. (2002), Une discipline pour la République: la science de l'éducation en France (1882-1914), Berne, Peter Lang.

Gelin D., Rayou P., Ria L. (2007), Devenir enseignant: parcours et formation, Paris, Armand Colin.

Hammersley M. (1999), Researching School Experience: Ethnographic Studies of Teaching and Learning, Londres - New York, Routledge.

Mons N. (2008), «Élites scolaires, inégalités sociales et renouveau des filières dans l'école moyenne: une comparaison internationale», Éducation et sociétés, n² 21.

Pollard A. (1982), The Social World of the Primary School, Londres, Holt, Rinehart and Winston.

VAN HAEсHT A. (1998), «Les politiques éducatives: figure exemplaire des politiques publiques?», Éducation et sociétés, n 1, p. 21-46. 


\section{PRATIQUER L'ANTHROPOLOGIE DE L'ENFANCE EN SCIENCES DE L'ÉDUCATION : UNE AIDE À LA RÉFLEXION}

Résumé: Pour traiter de la posture de l'anthropologue qui inscrit sa recherche en science de l'éducation, je m'appuierai pour une part sur mon expérience personnelle. J'ai reçu une formation universitaire d'anthropologie sociale et ethnologie et, au sein de ce cursus disciplinaire, j'ai choisi de travailler sur l'enfance, parcours qui m'a conduite à être aujourd'hui enseignante-chercheuse en sciences de l'éducation. Je tâcherai de poser quelques éléments qui présentent la position de l'anthropologie en sciences de l'éducation. Mais avant même cette présentation, c'est la position de l'objet enfance en anthropologie qui permettra de situer la problématique dans laquelle se trouvent les chercheurs qui, dans cette discipline, choisissent cet objet de recherche. Il s'agit en effet, d'une part, de distinguer la position de l'enfance dans ma discipline de formation et celle de l'anthropologie en sciences de l'éducation, d'autre part, d'examiner le cas particulier des anthropologues de l'enfance, chercheurs en sciences de l'éducation et de poser des questions transposables à d'autres disciplines accueillies par les sciences de l'éducation.

Mots clés: enfance, anthropologie, sociologie de l'enfance, décentrement, codisciplinarité.

\section{L'inégal prestige des objets d'une discipline: un sujet tabou}

\section{L'enfant: «petit sujet» de l'anthropologie}

Les questions d'enfance et d'éducation étaient, et demeurent en grande partie aujourd'hui, non seulement des sujets peu nobles en anthropologie, mais encore dévolus par tradition aux femmes. L'anthropologue américaine Margaret Mead avait déjà dénoncé cette hiérarchie des objets de recherche. Elle écrivait en 1972, à propos de son ex-époux lui aussi anthropologue, Réo Fortune: «Réo décida qu'il s’occuperait de la culture, et me laisserait la langue, les enfants, les techniques ${ }^{1}$.

1. Mead 1977, 210. 
Dans un article déjà ancien et fameux pour les chercheurs du champ, les anthropologues africanistes Suzanne Lallemand et Guy Le Moal présentaient eux aussi l'enfant comme "petit sujet» dans leur discipline ${ }^{2}$. Dans un article plus récent ${ }^{3}$, la première esquissait une histoire de l'anthropologie de l'enfance et notait l'intérêt plus grand de ses confrères pour cet objet dans la première partie du $\mathrm{XX}^{\mathrm{e}}$ siècle qu'aujourd'hui. Selon son analyse, actuellement, l'anthropologie de l'enfance balance entre une tendance extérieure à son champ propre (la psychologie du développement et de l'apprentissage) et des tendances plus conformes à ses orientations spécifiques (l'analyse des représentations symboliques de l'enfance, des rituels auxquels celle-ci donne lieu; l'examen des pratiques de soins de la prime éducation ainsi que les modalités d'insertion familiale et sociale du jeune individu).

Un récent numéro de la revue d'ethnologie Terrain intitulé «Enfant et apprentissage ${ }^{4}$ corrobore ses propos en exposant des articles relevant des deux registres. Parmi ceux-ci, celui de l'anthropologue américaine Lawrence A. Hirschfeld s'intitule «Pourquoi les anthropologues n'aiment-ils pas les enfants? » ${ }^{5}$. Selon l'auteure, la regrettable réticence des chercheurs de sa discipline à donner une place plus importante aux enfants résulte de ce qu'elle nomme deux erreurs: d'une part, une vision de l'enculturation qui surestime le rôle joué par les adultes dans les apprentissages culturels des enfants et minimise celui de ces derniers dans la reproduction de la culture. D'autre part, une méconnaissance de l'importance de la culture des enfants, en particulier dans son façonnage de celle des adultes. Ces lacunes pèsent sur l'analyse que nous faisons des phénomènes de transmission et de transformation des cultures, pourtant centrales dans la discipline.

Ces différents éléments montrent bien la position marginale et peu prestigieuse de l'anthropologie de l'enfance aux yeux de la discipline, malgré les défenseurs du champ. En fait, l'enfance est davantage étudiée indirectement à travers une anthropologie attentive aux manières d'éduquer la jeune génération. Les recherches interrogent l'action de l'adulte sur l'enfant pour le faire sortir de son état d'enfance et l'amener à l'âge adulte. Elles n'interrogent pas l'expérience des enfants et peu leurs relations entre eux.

\section{Les objets de l'anthropologie qui intéressent les sciences de l'éducation}

Si la recherche en anthropologie n'est pas la même quand elle est menée dans sa discipline ou bien en sciences de l'éducation, c'est déjà parce qu'elle suppose une sélection des objets qui intéressent celles-ci. Or c'est aujourd'hui l'anthropologie de l'éducation qui semble le champ de cette discipline le plus développé en sciences de l'éducation.

\footnotetext{
2. Lallemand \& Le Moal 1981.

3. Lallemand 2002.

4. Terrain 2003.

5. Hirschfeld 2003.
} 
Le champ a surtout traité des populations de territoires exotiques, dans le but de découvrir des modes d'éducation coutumière différents des nôtres ${ }^{6}$, mais aussi, dans les anciennes colonies, afin d'adapter la pédagogie occidentale au contexte local. L'œuvre de Pierre Erny est sans doute la plus révélatrice de cette double volonté. Son parcours montre comment, dans les années 1960 et suivantes, on pouvait conjuguer les disciplines. Lors d'une communication orale en septembre $2006^{7}$, il présentait sa carrière de la manière suivante. De formation philosophique, il a été instituteur dans une école de campagne dans l'actuel Burkina Faso à la fin de l'époque coloniale (1958) et plus tard au Rwanda, puis de retour en France il a reçu une formation en psychologie et ethnologie à l'université de Strasbourg. Dès qu'il a commencé à enseigner dans cette université, il a cherché à mettre les données ethnologiques à la disposition des pédagogues, des enseignants et des psychologues. Il a participé à la mise en place du programme des sciences de l'éducation dans son université. Il a ensuite enseigné dans des universités africaines, puis de retour en France il a enseigné la psychologie sociale puis l'ethnologie, toujours à Strasbourg. Il était donc en même temps du côté de la pédagogie et de l'ethnologie. Dans ses cours à l'université africaine, il était dans une problématique ethnopédagogique qui voulait adapter la pédagogie occidentale aux contextes culturels locaux grâce aux connaissances ethnologiques. Dans ses ouvrages, il a davantage développé l'ethnologie de l'éducation à partir d'une présentation du statut de l'enfant africain et de son éducation traditionnelle. Bien que l'ethnopédagogie ait été sa motivation de départ, il l'a finalement peu développée.

Ses travaux comportent donc une approche transdisciplinaire, alliant l'ethnologie de l'enfant et de l'éducation en Afrique (ce qu'il a appelé la païdologie, l'anthropologie appliquée à l'enfant ${ }^{8}$ ), l'approche sociologique et historique de l'école africaine comme institution, et l'approche psychologique de l'enfant et de l'adolescent. Il déplore qu'aujourd'hui, en ethnologie, il soit devenu un tabou de s'ouvrir à la pédagogie.

Son parcours amène à réfléchir sur les conséquences sur la recherche des frontières disciplinaires, telles qu'elles sont construites aujourd'hui institutionnellement, notamment à l'échelle d'un chercheur. Quelles en sont les conséquences sur sa manière de penser ses problématiques?

Sur ce point, inscrire sa recherche en sciences de l'éducation suppose une réflexion transdisciplinaire, quelle que soit la manière dont celle-ci se mette en œuvre, car la construction de cette « discipline pluridisciplinaire» suppose que toute question gagne à être abordée conjointement par plusieurs disciplines, chacune apportant ses questions, ses concepts et ses méthodologies.

\footnotetext{
6. Rabain 1979.

7. Erny 2006.

8. Erny 1972.
} 
L'anthropologie développe d'ailleurs, sur les thématiques éducatives, des recherches interdisciplinaires avec la psychologie notamment, par exemple pour étudier les ethnothéories parentales qui analysent les attentes et exigences des parents envers leurs enfants, en fonction des capacités qu'ils leur prêtent à un âge donné. Pour répondre aux interrogations de nos sociétés face aux difficultés rencontrées par l'école, aux États-Unis et en Europe notamment, elle a adapté ses théories, issues de l'anthropologie culturelle, afin d'analyser les situations des minorités ethniques à l'école et leur problème d'échec scolaire, ou encore pour comprendre les malentendus culturels entre enseignants et élèves. Elle a donc été sollicitée pour proposer des solutions aux difficultés pédagogiques rencontrées par les enseignants, et on a vu par exemple en France à partir des années 1980, dans les classes, la mise en place par certains enseignants de la pédagogie de l'interculturalité, pour faire face à ces difficultés.

L'anthropologie intéresse également les sciences de l'éducation pour sa méthodologie de l'enquête qui suppose une immersion sur le terrain et une observation participante. Ces outils méthodologiques, repris également par des chercheurs de disciplines voisines, notamment les sociologues, ont permis de développer une ethnographie de l'école attentive aux acteurs. Ils profitent aussi aux professionnels déjà en situation d'immersion qui utilisent ces techniques pour analyser leurs pratiques, comme en témoigne un numéro de la revue Spirale coordonné par Michelle Guigue et intitulé «Ethnographie de l'école " ${ }^{9}$. Le numéro de la revue Ethnologie française consacré à l'anthropologie de l'école et dirigé par Jean-Paul Filiod témoigne également de l'apport de l'anthropologie à l'univers scolaire, qui, au-delà de méthodes d'enquête, propose des outils conceptuels et des problématiques qui retravaillent des questions des sciences de l'éducation.

En accueillant l'anthropologie dans sa réflexion interdisciplinaire, les sciences de l'éducation permettent à celle-ci une valorisation nouvelle d'objets qu'elle considérait comme marginaux. Si jusqu'à présent les processus de développement, d'intégration et d'apprentissage ont mobilisé les recherches autour de l'enfance, les anthropologues ont dans leur discipline des outils conceptuels leur permettant de développer une recherche centrée sur l'enfance. Celle-ci permet de considérer les enfants comme un groupe social et suppose par exemple d'étudier, comme le fait Hirschfeld, la culture enfantine ${ }^{10}$. De manière plus globale, la démarche oblige à saisir le point de vue des enfants sur leur expérience sociale et non, tel qu'il a été classique de le faire, de s'enquérir du seul point de vue des adultes sur les sujets qui les concernent. Il reste alors aux anthropologues de l'enfance à montrer aux sciences de l'éducation l'intérêt d'une recherche ainsi centrée, intérêt que les chercheurs de leur discipline éponyme ne semblent pas prêts à reconnaître.

\footnotetext{
9. Spirale 2002.

10. Voir aussi Delalande 2006.
} 


\section{Cultiver les enrichissements réciproques initiés par la rencontre}

L'anthropologue qui inscrit ses recherches sur l'enfance en sciences de l'éducation voit son objet devenir légitime puisque les enfants font l'objet d'éducation. Le chercheur s'enrichit des nouvelles questions auxquelles les sciences de l'éducation le soumettent. Son inscription disciplinaire nouvelle lui permet d'envisager plus concrètement la pratique de recherche pluridisciplinaire. Nous essaierons d'explorer ces trois points.

\section{Des connaissances mises à l'épreuve: le regard décentré}

Pratiquer l'anthropologie de l'enfance dans sa discipline est une position relativement confortable, tout du moins est-elle classique. Il s'agit d'utiliser les outils et théories déjà élaborés par d'autres chercheurs pour penser son objet et développer des problématiques habituelles dans sa discipline, en utilisant les méthodologies d'enquête testées par ses prédécesseurs. La confrontation avec des chercheurs, des professionnels et des étudiants des sciences de l'éducation amène à un exercice très différent. Formés par d'autres disciplines, habités par d'autres questions et préoccupés par des situations professionnelles de terrain, ces acteurs perçoivent le regard de l'anthropologue sur l'enfance comme exotique. L'évidence des concepts de sa discipline éponyme ne fonctionne plus et il doit en démontrer la validité pour penser des situations concrètes auxquelles il n'avait pas songé. Plus que le passage d'une anthropologie fondamentale à une anthropologie appliquée, la difficulté réside dans le fait d'un anthropologue qui s'applique à penser des questions qui ne sont pas les siennes sur un objet de recherche qu'il croit connaître. Ce regard décentré sur l'enfance, préoccupé par des questions pédagogiques notamment, met à l'épreuve ses constructions théoriques. Ces dernières résistent-elles aux expériences des professionnels de l'enfance et aux autres analyses disciplinaires?

Dans cette rencontre, il est difficile de démêler les apports de chacun pour l'autre. Pour ma part, le regard des acteurs des sciences de l'éducation sur ma recherche (j'entends les chercheurs, professionnels et étudiants) m'amène à constater que mes analyses les intéressent pour des raisons qui ne sont pas les miennes. De mon point de vue, mes études me permettent de décliner l'idée de groupe social et culturel pour penser une population enfantine et d'étudier des concepts classiques de ma discipline, comme celui de culture et de transmission culturelle, d'identité sociale ou encore celui du don et de son rôle dans les échanges, à l'échelle du groupe d'enfants auprès duquel je travaille. Ma problématique demeure donc, ou devrais-je dire demeurait, car précisément le contact avec ma discipline d'accueil enrichit mon regard, celle d'une monographie d'un groupe défini, en cherchant à voir ce qui découle de la spécificité de cette population qui se trouve dans une relation de dépendance avec des adultes.

Pour les acteurs des sciences de l'éducation, mes analyses permettent de poser un autre regard sur l'être à éduquer, en approfondissant ce qui se joue entre enfants, autrement dit en sortant d'un «adultocentrisme » qui réduit les questions d'éducation 
et de socialisation à celles des relations verticales de l'adulte vers l'enfant. Mes études ethnologiques approfondissent l'expérience sociale et culturelle des enfants, grâce à des enquêtes qui consistent à saisir leur point de vue par observation et entretien, et posent par exemple un regard sur l'école comme lieu de vie entre pairs sans s'attacher à la mission d'instruction de l'institution scolaire. Mon approche anthropologique de l'enfance permet aux étudiants de relativiser leur conception de cet âge de la vie en travaillant les représentations sociales et manières d'éduquer les enfants dans d'autres régions du monde et antérieurement dans nos pays. Les cours que je leur dispense m'obligent à dépasser l'objectif de transmettre les concepts clés de ma discipline pour rendre concrets, en fonction de leurs objectifs professionnels, les enjeux du relativisme culturel et historique. De mon côté, ce dépassement modifie profondément le sens que j'attribue à mon enseignement et de ce fait la manière dont je le conçois.

En conséquence, l'intérêt que les acteurs des sciences de l'éducation trouvent à ma recherche m'oblige à sortir des seuls enjeux conceptuels tels que ma discipline m'amène à les concevoir. Des problématiques nouvelles émergent de notre rencontre qui ne sont ni celles de ma discipline ni celles que ma recherche inspire à mes collègues et étudiants. Elles sont une ouverture pour nous tous. Elles interrogent par exemple les conséquences d'une meilleure connaissance pour les professionnels de l'enfance, enseignants ou éducateurs, des relations entre enfants, sur leur travail auprès d'eux. Elles enrichissent plus fondamentalement les réflexions sur l'évolution de nos représentations de l'enfance et celle du statut de l'enfant dans notre société. Elles appellent à une réflexion conjointe avec les étudiants et à des analyses codisciplinaires entre chercheurs.

\section{Acquérir et nourrir une culture commune en gardant sa spécificité}

Construire une analyse codisciplinaire sur un objet suppose de partir d'une culture disciplinaire commune facilitant la discussion. Un des intérêts des sciences de l'éducation est, pour un chercheur formé par une autre discipline, de proposer cette «culture commune ${ }^{11}$ qui s'ajoute à l'héritage disciplinaire de chacun. Celle-ci suppose une "socialisation identitaire disciplinaire » ${ }^{12}$, basée sur la connaissance des auteurs, ouvrages, concepts et théories de référence, mais fondée aussi sur le rassemblement de chercheurs originaires de disciplines diverses au sein de laboratoires de recherche. Cette socialisation par immersion suppose aussi d'être attentif au « regard décentré» que proposent les collègues, tel que je l'ai présenté dans le point précédent. Elle se fait au sein des laboratoires mais peut aussi être travaillée par la proposition d'enseignements qui tentent une approche plurielle d'un objet. L'expérience, au sein de mon équipe, d'un cours de master première année d'approche codisciplinaire, suppose un plaisir à se confronter à la difficulté d'élaborer de concert. Elle permet d'avoir des témoins, les étudiants, de la pertinence visible ou non d'une approche plurielle

11. Charlot 1998.

12. Peyronie 2002. 
sur une question et oblige surtout à un travail pédagogique pour expliciter cette pertinence. Plus fondamentalement, c'est au sein d'une recherche commune que l'intérêt d'une approche codisciplinaire apparaît.

$\mathrm{Si}$ je devais approfondir ce que suppose l'immersion dans ladite culture commune, j'emploierais une image typiquement anthropologique dans laquelle ma position est celle de l'immigré, ni tout à fait le même, ni tout à fait un autre, qui doit s'intégrer sans s'assimiler, puisqu'il est riche du fait de ses origines et qu'il est ici dans un pays d'accueil particulièrement hospitalier. C'est en effet les sciences de l'éducation qui permettent ma reconnaissance en tant qu'anthropologue et non ma discipline mère.

\section{Les sciences de l'éducation : une expérience d'analyse pluridisciplinaire transposable à d'autres objets?}

La position de l'objet «enfance» peut-elle être comparée à celle de l'objet «éducation » ? Pratiquer l'anthropologie de l'enfance au sein des sciences de l'éducation incite à concevoir une approche pluridisciplinaire de l'objet. Celui-ci pourrait-il être étudié par des «sciences de l'enfance», réunies dans l'optique d'une réflexion commune sur un objet en évolution? Cet objet, peu légitime en anthropologie, mais peu valorisé également en sociologie, dans laquelle il a longtemps été abordé à partir d'une sociologie de la famille, de l'école ou une sociologie de l'éducation, peut devenir plus légitime si plusieurs disciplines l'étudient de concert.

C'est ce que tentent depuis quelques années un groupe de chercheurs francophones en sciences sociales et humaines, réunis au sein d'un comité de recherche de l'AISLF (Association internationale des sociologues de langue française), le CR 31 «Sociologie de l'enfance ${ }^{13}$. La reconnaissance institutionnelle du groupe permet une valorisation de l'objet de recherche. Mais elle camoufle une dimension qui donne pourtant sa dynamique aux échanges scientifiques qui s'y produisent, celle de la rencontre interdisciplinaire. Ici, la diversité des disciplines disparaît derrière la bannière de la sociologie. La rencontre entre disciplines est en partie dépendante des institutions qui la permettent et sans doute cette rencontre est-elle encore peu instituée, exception faite précisément de l'histoire des sciences de l'éducation qui institue une bannière composite, et des demandes de projets de recherche autour d'une équipe pluridisciplinaire.

L'histoire des sciences de l'éducation doit aider à penser tout nouveau regroupement de chercheurs de diverses origines autour d'un objet. Ce groupe doit chercher à se prémunir de deux dangers: celui de gommer les différences d'approches disciplinaires sur l'objet. La recherche d'une culture commune ne devrait pas annuler la richesse de chaque discipline, mais au contraire, obliger chacune d'elles à réinterroger ses théories et à bousculer celles des autres. Le deuxième danger est la mise en avant d'une discipline qui prend une position dominante face aux autres et brise la dynamique d'échange.

13. Sirota 2006 . 
L'histoire des sciences de l'éducation peut également nous mettre en garde contre le danger d'une migration des termes et de leur sens, résultat d'effets de mode sociétaux dans laquelle s'engouffre la recherche. Une première migration a eu lieu, dans la valorisation d'une sociologie de l'enfance, qui souhaite se différencier d'une sociologie de l'éducation. Simple effet de mode qui résulte d'une mise en avant de l'enfance dans notre société ${ }^{14}$ ? Si l'expression répond à un désir, dont on peut se réjouir, de mieux connaître l'enfance au-delà de la relation éducative avec l'adulte, instituer une sociologie de l'enfance répond-il à ce besoin? Dans la mobilisation des chercheurs du groupe «sociologie de l'enfance» de l'AISLF, il semble que l'on risque de ne pas répondre au besoin ressenti en ne se posant pas les questions que celui-ci soulevait. Car c'est sans doute par l'approche plurielle que les questions des chercheurs sur l'enfance trouveront à se nourrir.

L'histoire des sciences de l'éducation nous apprend encore que l'approche pluridisciplinaire permet non seulement de nouveaux éclairages sur l'objet travaillé, mais enrichit également chacune des disciplines mères qui y participent. Sur ce point, l'objet qui réunit l'attention de chercheurs de plusieurs disciplines peut occuper dans leurs travaux plusieurs positions. Soit il est une sorte de "vache à lait » permettant l'approfondissement d'autres questions jugées plus fondamentales. L'étude de l'enfant peut être un moyen de mieux comprendre la construction de l'adulte et la manière dont il se socialise lors de ses premières années. Elle peut être un moyen de comprendre le phénomène d'acquisition du langage ou celui du développement des fonctions cognitives. Dans ce cas, constituer des «sciences de l'enfance» semble artificiel puisque l'intérêt pour l'objet disparaît quand le phénomène est compris. Si au contraire l'ambition des chercheurs est de fonder une réflexion transdisciplinaire sur l'enfance avec comme objectif une meilleure connaissance de l'objet, qui répond également aux interrogations de leur société, la constitution de «sciences de l'enfance» permet une immersion dans l'objet et impulse une dynamique de reconnaissance institutionnelle.

\section{Les sciences de l'éducation : discipline mère pour de jeunes docteurs?}

Je terminerai sur une question qui dépasse mon propos. Qu'en est-il des chercheurs qui ont suivi leur cursus dans un département de sciences de l'éducation jusqu'à leur thèse, sans se rattacher clairement à une discipline mère? Doivent-ils se nourrir de la culture commune des sciences de l'éducation ou doivent-ils «s'inventer» une discipline mère? Autrement dit: peut-on être dans un cursus de sciences de l'éducation sans autre discipline mère que celle-ci? A-t-elle le même statut qu'une autre discipline ou bien demeure-elle un regroupement disciplinaire autour d'un objet, comme on pourrait l'imaginer d'une «science de l'enfance»? Il semble que jusqu'à présent les jeunes docteurs formés en sciences de l'éducation choisissent un rattachement à une

14. Danic et al. 2006, 10. 
discipline mère dans laquelle ils approfondissent par eux-mêmes leur formation et qui est fréquemment la discipline dans laquelle leur directeur de recherche se reconnaît. Dans ce cas, leur connaissance de leur discipline d'appui est-elle la même qu'un étudiant qui aurait suivi le cursus de celle-ci? Ne sont-ils pas tentés de ne sélectionner dans leur exploration que les auteurs et théories centrés sur leur objet de recherche? Armés d'une compétence moindre dans la discipline de rattachement qu'ils ont élue, ne manquent-ils pas d'aisance à manier les concepts et outils de cette discipline qui devraient nourrir leurs analyses? Sans doute serait-il important pour les sciences de l'éducation de mener à ce propos une recherche approfondie, afin d'éclairer non seulement leur devenir, mais aussi celui de disciplines voisines qui se nourrissent de l'expérience des sciences de l'éducation pour mener leurs recherches pluridisciplinaires.

Julie DeLalande

CERSE

Université de Caen Basse-Normandie

\section{Références bibliographiques}

Charlot B. (1998), «Les sciences de l'éducation en France: une discipline apaisée, une culture commune, un front de recherche incertain", in Le pari des sciences de l'éducation, R. Hofstetter, N. Schneuwly (dir.), Bruxelles, De Boeck (Raisons éducatives), p. $147-167$.

Danic I., Delalande J., Rayou P. (2006), Enquêter auprès d'enfants et de jeunes, objets, méthodes et terrains en sciences sociales, Rennes, Presses universitaires de Rennes.

Delalande J. (2006), "Le concept heuristique de culture enfantine», in Éléments pour une sociologie de l'enfance, R. Sirota (dir.), Rennes, Presses universitaires de Rennes, p. 267-274.

ERny P. (1972), L'enfant et son milieu en Afrique noire. Essai sur l'éducation traditionnelle, Paris, Payot.

ERny P. (2006), Discours de conclusion des $6^{e}$ journées d'études internationales du comité de recherche $n^{\circ} 31$ «Sociologie de l'enfance» de l'AISLF (Association internationale des sociologues de langue française), Les enfants: "un monde à part»? Altérité de l'enfant et de l'enfance dans les pratiques de recherche en sciences sociales, Université Marc Bloch, Faculté des sciences sociales, pratiques sociales et développement, Strasbourg, 14-15 septembre 2006.

Filıod P. (dir.) (2007), «Anthropologie de l'école. Perspectives », Ethnologie française, vol. 37 . 
Hirshfeld L.A. (2003), «Pourquoi les anthropologues n'aiment-ils pas les enfants?», Terrain, $\mathrm{n}^{\circ} 40$, p. 21-48.

LALLEMAND S. (2002), « Esquisse de la courte histoire de l'anthropologie de l'enfance, ainsi que de certains de ses thèmes électifs", Journal des Africanistes, vol. 72, n 1, p. 9-18.

Lallemand S., Le Moal G. (1981), «Un petit sujet», Journal des Africanistes, vol. 51, n 1-2, p. 5-21.

Mead M. (1977), Du givre sur les ronces, Paris, Seuil.

Peyronie H. (2002), « Les sciences de l'éducation, une discipline? Logique institutionnelle et logique épistémologique: une relation dialectique», in Les sciences de l'éducation. Des recherches, une discipline, J.-F. Marcel (dir.), Paris, L'Harmattan, p. 177-196.

Rabain J. (1979), L'enfant du lignage. Du sevrage à la classe d'âge chez les Wolof du Sénégal, Paris, Payot.

Sirota R. (dir.) (2006), Éléments pour une sociologie de l'enfance, Rennes, Presses universitaires de Rennes.

Spirale (2002), «Ethnographie de l'école», M. Guigue (coord.), n³0.

Terrain (2003), «Enfant et apprentissage», G. Lenclud (coord.), n 40. 


\section{LES RECHERCHES EN SCIENCES DE L'ÉDUCATION ET DANS LES DISCIPLINES ÉPONYMES}

- L'histoire - 



\section{TERRITOIRES ET PRATIQUES DE L'HISTOIRE DE L'ÉDUCATION \\ Le point de vue d'un historien des $\mathrm{XIX}^{\mathrm{e}}$ et $\mathrm{XX}^{\mathrm{e}}$ siècles}

Résumé: L'histoire de l'éducation est longtemps restée pour les historiens un domaine de second ordre, mais les analyses de Jean-Noël Luc montrent que la situation est en train de changer : thèses, revues, publications... en témoignent. Il propose alors un inventaire détaillé des territoires où elle se développe: histoire culturelle, histoire des institutions religieuses, histoires des établissements scolaires et des champs de la formation (enseignement technique, éducation populaire...), histoire des enseignants et des populations d'élèves, histoire de la culture et des savoirs scolaires. Pour l'historien de l'éducation, les questions croisent souvent d'autres recherches et ouvrent à d'autres sciences humaines et sociales; elles l'obligent à étudier des processus nouveaux, à faire appel à d'autres concepts, à s'intéresser à des objets inédits (manuels, pratiques pédagogiques, identités professionnelles...). L'histoire de l'éducation trouve ainsi sa fécondité et sa richesse dans la pluralité et la transversalité de ses curiosités.

Mots clés: histoire de l'éducation, histoire de l'enfance, histoire sociale.

«Laissez ce sujet à une femme et prenez un beau sujet d'histoire politique»! L'assistant d'histoire destinataire de ce conseil amical, en 1979, venait d'annoncer qu'il envisageait de préparer un doctorat d'État sur la préscolarisation au XIX ${ }^{\mathrm{e}}$ siècle. Mais pour l'éminent professeur non contemporanéiste qui s'intéressait à son destin, l'éducation - surtout celle des petits, domaine féminin par excellence - constituait un thème de recherche secondaire et, qui plus est, inopérant pour favoriser une carrière universitaire. Vingt ans plus tard, l'ancien assistant devenu professeur entendait un collègue de province chevronné le féliciter d'être "sorti de l'éducation» - ce qui était faux pour mieux s'investir dans l'histoire de la Gendarmerie et des gendarmes! Ces deux anecdotes témoignent de la faible considération accordée à l'histoire de l'éducation par une partie de la corporation historienne. Faut-il voir dans ce discrédit un réflexe de méfiance, classique à l'Université, à l'égard d'un thème de recherche et d'enseignement soupçonné - à tort - de contribuer à privilégier le mode de transmission du savoir sur le savoir lui-même? D’autres indices révèlent, au contraire, sa promotion graduelle au sein de la communauté scientifique. 


\section{L'essor d'un champ de recherche depuis la fin des années 1970}

Plusieurs pionniers ont ouvert la voie entre la fin des années 1940 et celle des années 1960: en histoire, Henri-Irénée Marrou, Philippe Ariès, Pierre Riché, Paul Gerbod ou Antoine Prost, en sociologie ou dans d'autres disciplines, Antoine Léon, Georges Snyders, Guy Avanzini ou Viviane Isambert-Jamati, pour ne citer qu'eux. Dès 1961, dans un ouvrage de référence, L'histoire et ses méthodes, le médiéviste Georges Duby juge d'ailleurs indispensable, pour l'histoire des mentalités, "une étude préalable des institutions scolaires, de leurs structures, de leurs méthodes, des notions qu'elles veulent transmettre, de leur équipement, de leur implantation dans la société, de ce qui les rattache aux autres cadres, familiaux, militaires, religieux, politiques ${ }^{1}$. Mais ces initiatives restent isolées, et l'histoire de l'éducation ne figure pas dans les deux bilans de la recherche historique publiés en 1974 (Faire de l'histoire et Aujourd'hui l'histoire).

La situation change au cours des années suivantes. Un plus grand nombre de travaux fondateurs sont alors publiés, comme l'enquête de François Furet et de Jacques Ozouf sur L'alphabétisation des Français, de Calvin à Jules Ferry (1978) ou la synthèse de l'Histoire générale de l'enseignement et de l'éducation en France (1981-1982), tandis que le service d'histoire de l'éducation, intégré en 1977 au sein de l'Institut national de recherche pédagogique (INRP) lance la revue Histoire de l'éducation. L'année suivante, le manifeste de La nouvelle histoire accorde à l'éducation une rubrique autonome. La croissance durable des publications consolide ce mouvement. La proportion d'articles de la Revue d'histoire moderne et contemporaine relatifs à l'histoire de l'éducation sous toutes ses formes passe de $2 \%$ du total entre 1954 et 1963 à 7,1 \% entre 1974 et $1987^{2}$. En 1988, la responsable de la bibliographie annuelle de l'histoire de France souligne «l'extraordinaire essor de l'histoire de l'enseignement», qui affiche le plus fort taux de croissance $(+354 \%)$ en trente ans ${ }^{3}$. Dans ce contexte favorable, les éditions Belin lancent, en 1997, une collection «Histoire de l'éducation », dirigée par Bruno Belhoste, Pierre Caspard, Jean-Noël Luc et Antoine Prost.

Plusieurs facteurs expliquent le dynamisme de ce champ de recherche au sein d'une histoire contemporaine elle-même foisonnante. L'essor de l'histoire culturelle, le renouvellement de l'histoire sociale et le développement de l'histoire de la famille, de l'enfance et de la jeunesse attirent l'attention sur les modes de transmission des valeurs et des savoirs. La variété des structures éducatives - famille, école, Églises, mouvements de jeunesse, organisations d'éducation populaire, armée, entreprises favorise la multiplication des travaux. Les orientations des autres sciences humaines et sociales inspirent des investigations sur le rôle de l'école dans certains processus politiques et sociaux, comme la surveillance, la normalisation et la reproduction. Enfin, plusieurs conjonctures se succèdent ou s'entremêlent pour entretenir la demande

\footnotetext{
1. Samaran $1961,958$.

2. Caspard 1988, 10.

3. Havelange 2002, 61.
} 
sociale d'histoire: disparition de «l'école de Jules Ferry» dans les années 1960, avènement progressif de la scolarisation de masse, génératrice de nouveaux problèmes, accumulation des commémorations à partir de 1981. Or, le vivier des spécialistes académiques répond à cette attente autant qu'il la nourrit, puisqu'il rassemble, à côté des historiens, des chercheurs venus d'horizons divers, et notamment des sciences de l'éducation, institutionnalisées dans l'Université à partir de 1967.

\section{Un chantier pluridisciplinaire}

La répartition des thèses par discipline témoigne de cette diversité. Si $40 \%$ d'un corpus de 290 thèses françaises soutenues entre 1991 et 1995 sur l'histoire de l'éducation, toutes périodes confondues, relèvent de l'histoire, les autres se répartissent entre la médecine (19\%), les sciences de l'éducation (14\%), la littérature et la linguistique $(13 \%)$, la sociologie $(5 \%)$ et d'autres disciplines $(10 \%)^{4}$.

Les chercheurs de formation historienne constituent le groupe le plus nombreux. À la fin du $\mathrm{XX}^{\mathrm{e}}$ siècle, on a estimé à 200 environ ceux qui travaillent et publient plus ou moins régulièrement sur l'histoire de l'éducation depuis l'Antiquité. C'est dire que les sous-entendus péjoratifs évoqués au début de ce texte doivent être un peu relativisés, et d'autant plus qu'ils sont vraisemblablement moins répandus chez des chercheurs jeunes ou influencés par l'histoire sociale et culturelle. Les spécialistes des sciences de l'éducation constituent les seconds acteurs du développement de l'histoire de l'éducation en France, alors que les facultés dites de pédagogie, dotées de multiples chaires d'histoire spécialisées, jouent un rôle prépondérant dans les nombreux pays européens fidèles au modèle germanique ${ }^{5}$. Mais si les instituts français de sciences de l'éducation comptent peu de postes de ce type, et d'historiens de formation, l'histoire y est présente, depuis l'origine, dans la réflexion ${ }^{6}$, la recherche (les exemples sont trop nombreux pour être inventoriés ici), l'enseignement et les publications. Au cours des années 1981-1985, 12 \% des thèses soutenues en sciences de l'éducation traitent d'un sujet d'histoire ou possèdent une dimension historique ${ }^{7}$. Par ailleurs, des articles historiques paraissent régulièrement dans la Revue française de pédagogie, Carrefours de l'éducation et Le Télémaque. Une troisième catégorie de professionnels rassemble des spécialistes venus d'horizons divers, mais intéressés par la genèse de leur discipline, les processus de son institutionnalisation et l'évolution de son enseignement.

L'histoire de l'éducation s'est aussi développée grâce à l'action du Service d'histoire de l'éducation (SHE) de l'INRP, unité de recherche associée au CNRS (section Histoire moderne et contemporaine), puis équipe associée à l'ENS. Sous la direction,

\footnotetext{
4. Havelange 2002, 72.

5. Compère 1995, 24.

6. $\quad$ Debesse \& Mialaret 1971-1972; Plaisance \& Vergniaud 1993, 61-68.

7. Caspard 1988, 15 .
} 
depuis trente ans, de Pierre Caspard, spécialiste d'histoire sociale et culturelle, ce service a contribué à structurer la recherche aux côtés des universités par la production d'instruments de travail, dont la Bibliographie annuelle de l'histoire de l'éducation, la publication de numéros thématiques de la revue Histoire de l'éducation, l'ouverture de pistes neuves, l'organisation de séminaires et de colloques ${ }^{8}$.

Après ce premier survol, comment définir le champ et la pratique de l'histoire de l'éducation? Les réponses proposées ici restent subjectives, puisqu'elles s'appuient sur la seule bibliographie connue de l'auteur, sur une expérience de chercheur en histoire sociale et culturelle et sur la direction, depuis dix ans à la Sorbonne, d'un séminaire d'histoire contemporaine - Enfance, jeunesse, éducation et société, XIX ${ }^{e}-X X^{e}$ siècles au sein duquel ont été soutenus, à la fin de l'année 2007, 125 maîtrises, DEA ou masters, 7 doctorats et 4 HDR.

\section{Territoires et objets d'étude}

Les principales orientations de la recherche renvoient à une dizaine de grands thèmes d'étude. L'histoire de l'éducation est d'abord celle d'un projet culturel. «Pour toute humanité, enseigner, au fond, c'est s'enseigner », affirmait Charles Péguy en 1904. En filigrane des doctrines pédagogiques, des objectifs de l'école, des programmes et de leurs commentaires, on entrevoit, sous un angle original, les valeurs, les savoirs, les aspirations, mais aussi les préjugés et les peurs d'une société. C'est pourquoi l'histoire de l'éducation a profité de l'essor d'une histoire culturelle soucieuse d'étudier, notamment, «un monde légué par les transmissions dues au milieu, à l'éducation, à l'instruction " ${ }^{9}$.

Une seconde approche globale souligne la diversité des processus de formation et le retard de l'histoire de l'éducation non scolaire. Le dynamisme des recherches sur les institutions d'enseignement, qui orientent la réflexion présentée ici, ainsi que sur les mouvements de jeunesse, l'éducation surveillée ou la littérature enfantine et de jeunesse, ne doit pas masquer le déficit de travaux sur d'autres formes ou d'autres lieux d'éducation: famille, établissements pour handicapés, éducation populaire, groupes de jeunes, armée, apprentissage, formation continue.

Objet politique, l'éducation sous toutes ses formes trouve sa place dans un champ de recherche qui s'est depuis longtemps renouvelé. De nombreuses mutations du système éducatif ont donné lieu à des affrontements, entre l'État et l'Église romaine, les partis, les groupes de pression, les syndicats. Au-delà des discours, on commence aussi à s'intéresser - insuffisamment, hélas - aux contributions de l'État, des collectivités locales et des familles au financement de l'école, qui mériteraient d'être étudiées - en ce qui concerne les fonds publics - jusqu'au niveau des crédits réellement dépensés.

\footnotetext{
8. Caspard 2006.

9. Sirinelli 1992, III.
} 
Ces premiers terrains d'investigation rappellent que l'éducation est l'un des modes d'intervention privilégiés des Églises dans la société. La contribution des institutions religieuses à la scolarisation, la succession des guerres scolaires et les nouveaux débats apparus à la fin du $\mathrm{XX}^{\mathrm{e}}$ siècle justifient une présentation autonome de ce chantier, où plusieurs pistes peuvent être distinguées: formation interne des clercs et des fidèles, établissements d'enseignement confessionnels, intervention des congréganistes dans le système scolaire public, œuvres péri- ou post-scolaires, conflits avec l'État et une partie de la société, notamment à propos de la laïcisation ou de la laïcité.

En réaction à une histoire trop attachée aux idées pédagogiques ou aux décisions des pouvoirs centraux, plusieurs historiens ont déplacé leur regard vers la cellule de base de l'épithélium scolaire: l'établissement. Ils ne renouent pas pour autant avec le genre monographique traditionnel, puisqu'ils considèrent l'établissement scolaire comme objet d'histoire totale, c'est-à-dire non seulement comme ensemble de bâtiments, unité de gestion, espace de formation et de vie, collectivité d'adultes et d'élèves, mais aussi comme élément d'un réseau d'institutions rivales ou complémentaires, employeur et client de l'économie locale et lieu d'interactions entre tous les acteurs locaux de la scolarisation : administration scolaire, municipalités, conseils généraux et régionaux, associations diverses, dont celles des parents d'élèves, et entreprises ${ }^{10}$. Parmi les sujets promus par ce renouveau d'intérêt pour l'institution scolaire elle-même, on peut citer les enseignements techniques et intermédiaires, très dépendants des initiatives locales, «l'offre effective de places » dans la scolarisation post-élémentaire, l'architecture des bâtiments, les politiques scolaires des grandes villes et, nous y reviendrons, la culture scolaire et son acquisition.

L'approche historique s'intéresse aussi aux deux grands acteurs de l'entreprise éducative: les professionnels auxquels la collectivité délègue la mission d'éduquer ou de faire fonctionner les institutions, et les publics visés. Les enseignants sont les protagonistes les mieux observés, dans leurs trajectoires socioprofessionnelles, leur recrutement, leur professionnalisation, leurs engagements et leurs images. Longtemps négligés, les administrateurs sortent de l'ombre, du moins certains d'entre eux, comme les inspecteurs primaires, les inspecteurs généraux et, récemment, les recteurs, objets d'un travail d'histoire totale pour les années 1808-1940. Il en est de même des enseignantes, de plus en plus étudiées à la faveur de l'essor de l'histoire du genre et de la professionnalisation féminine ${ }^{11}$. Dans la cohorte des oubliés, restent les agents des services administratifs de tout niveau, les chefs d'établissement, les personnels nonenseignants et les parents d'élèves, dont le rôle, au travers des diverses associations, déborde la seule notion de "demande sociale».

L'histoire des élèves est un puzzle encore moins achevé, surtout si l'on s'écarte des profils et des cursus, eux-mêmes inconnus pour plusieurs catégories d'entre eux. Or les attitudes des enseignés, en classe, en récréation, à l'internat, donc leur histoire

10. Compère \& Savoie 2001 et 2005.

11. Rogers 2007. 
scolaire et personnelle, déterminent aussi la forme et les effets de l'acte éducatif. À titre d'exemples de pistes de recherche, on citera les comportements des élèves (notamment dans le cadre des interrelations entre tous les acteurs de la communauté scolaire), la construction de leurs identités sexuées, leur perception de l'institution scolaire, les représentations dont ils sont l'objet et les effets de leur formation sur leur cursus ultérieur, leur insertion professionnelle et leur trajectoire sociale.

Dans le champ de référence choisi ici, l'enquête se poursuit autour de la culture scolaire et de sa transmission. Trois thèmes d'étude - savoirs enseignés, pratiques éducatives et résultats - se dégagent des travaux qui se multiplient, depuis une vingtaine d'années, au Service d'histoire de l'éducation (SHE), en sciences de l'éducation et, grâce à des didacticiens parfois en poste dans les IUFM, au sein de plusieurs disciplines ${ }^{12}$. André Chervel a joué un rôle fondateur dans l'émergence et l'orientation de l'histoire des «disciplines scolaires" au sein de ce nouveau chantier. Dès 1988, ce chercheur du SHE affirme l'autonomie relative de l'école par rapport aux injonctions officielles, et sa capacité à produire une culture spécifique, faite d'emprunts à la culture savante, mais aussi de savoirs, de méthodes et d'exercices créés pour les besoins de l'apprentissage et de la hiérarchisation des élèves ${ }^{13}$. Cette analyse stimulante a inspiré plusieurs recherches sur la construction institutionnelle de certaines matières, les composantes de la transposition didactique (programmes officiels, culture professionnelle des enseignants, contenu et usage des manuels, pratique du cours magistral) et les travaux d'élèves. Trop souvent perdue, cette dernière source est l'une des rares qui permettent d'apprécier, à la fois, l'interprétation magistrale des compétences attendues, son influence sur l'enseignement et les acquisitions effectives.

L'histoire n'oublie pas non plus de traiter les institutions éducatives et leurs messages comme des lieux de mémoire, dont l'étude peut être entreprise sur trois niveaux. La mémoire collective de l'école s'exprime surtout à travers des bâtiments, des musées, les discours de quelques directeurs d'opinion et la vulgate transmise par les médias. La mémoire individuelle ou commune de certains acteurs est conservée dans des autobiographies et des bulletins d'amicales ou sauvegardée grâce à des enquêtes écrites ou orales. Ces deux mémoires livrent à l'historien des informations originales, grâce auxquelles il comprend mieux le fonctionnement du système scolaire et les comportements de ses personnels et de ses usagers. Mais elles diffusent aussi une vision autonome du passé de l'école, parfois imprégnée de nostalgie, commercialement rentable, ou biaisée par le poids des idéologies, voire pour les besoins d'interprétations partisanes.

Ces dernières transparaissaient déjà dans des travaux d'histoire de l'éducation réalisés, entre le milieu du XIX siècle et les années 1930, dans un contexte de guerre scolaire vive ou sporadique. Tandis que les cléricaux accusaient la Révolution puis les républicains d'avoir ruiné l'entreprise d'éducation conduite par l'Église romaine, les

12. Compère \& Savoie 2005; Caspard 2006, 20-40.

13. Chervel 1988 et 1998. 
laïcs dénonçaient l'obscurantisme de l'Ancien Régime pour mieux exalter les réalisations du XIX ${ }^{e}$ siècle, couronnées par celles de la Troisième République. Cette seconde lecture a été suffisamment prégnante pour empêcher, pendant longtemps, les historiens contemporanéistes de l'éducation de prendre en considération l'œuvre des communes avant la loi Guizot ou le développement de certaines filières (préscolarisation, post-élémentaire féminin, primaire supérieur, technique) avant les initiatives de la Troisième République, au demeurant importantes même quand elles ne sont pas fondatrices. L'audience récente du «devoir de mémoire» a donné un nouveau souffle à la mobilisation des mythes de l'école républicaine au service du débat récurrent sur le malaise du système scolaire ${ }^{14}$. L'école "d'autrefois» est convoquée pour vilipender l'intérêt, baptisé "pédagogisme», porté aux modes de transmission et d'acquisition du savoir, les projets de sélection ou les responsabilités de l'école d'aujourd'hui dans la "panne de l'ascenseur social». C'est dire que les contradictions entre l'instrumentalisation du passé et les résultats de la recherche peuvent constituer un autre objet d'étude.

Lorsque l'obstacle linguistique n'existe pas, plusieurs des travaux évoqués ici peuvent être conduits au-delà des frontières nationales. L'histoire de la circulation internationale des modèles institutionnels ou pédagogiques éclaire les filiations et leurs multiples relais, mondains, confessionnels, associatifs, éditoriaux ou professionnels. L'étude précise des systèmes éducatifs nationaux fait ressortir, au contraire, leur spécificité, façonnée par des contextes - économiques, sociaux, politiques, culturels - particuliers. Malgré les contributions de certains spécialistes de langues et civilisations étrangères, et celles des doctorants étrangers étudiant le cas de leur propre pays, ces chantiers sont peu développés: l'histoire de l'éducation à l'étranger et l'histoire comparée des faits éducatifs demeurent des points faibles de l'historiographie française.

Quel que soit l'objet d'étude, l'approche historienne de l'éducation reste fidèle aux exigences heuristiques de la discipline de référence. Faute de pouvoir la décrire ici dans son ensemble, on a choisi de s'inspirer des conseils dispensés au sein du séminaire déjà évoqué pour présenter quelques pratiques jugées les plus pertinentes.

\section{Une enquête exigeante}

Si la construction de tout objet historique requiert un questionnement, le choix d'un sujet, au niveau du master comme du doctorat, impose aussi aux directeurs de recherche de se préoccuper de la documentation disponible. L'histoire de l'éducation recourt aux matériaux habituels de la recherche moderne (archives, sources imprimées, iconographiques, audiovisuelles et orales, objets), remarquablement inventoriés, surtout à propos du système scolaire, dans un guide du chercheur ${ }^{15}$. Lorsque le sujet s'y prête, les archives doivent être considérées comme des documents fondamentaux. Là plus

14. Compère \& Savoie 2005, 109; Caspard 2008.

15. Charmasson 2006. 
qu'ailleurs, se trouvent en grand nombre des indices sur la genèse d'une décision officielle, l'influence réelle d'une théorie pédagogique, les singularités du fonctionnement d'un établissement, les effets de l'enseignement, les événements contingents. Ni leur volume, ni leur dispersion, ni le coût horaire de leur consultation, ne doivent rebuter le chercheur: comme les autres champs historiques, l'histoire de l'éducation ne se construit pas à l'économie.

Deux autres pratiques contribuent à sa spécificité: l'insertion de ses objets d'étude dans les dynamiques générales - économiques, démographiques, sociales, politiques, culturelles, religieuses - d'une société, et la construction de leur historicité. Le traitement de la préscolarisation comme un fait social complexe a été, par exemple, le seul moyen de découvrir l'articulation - différente selon les périodes, les lieux et les individus - de tous les facteurs associés à la multiplication des écoles enfantines au XIX ${ }^{\mathrm{e}}$ siècle $^{16}$ : ferveur éducative héritée du piétisme ou des Lumières, nouvelle politique d'assistance, reconquête religieuse post-révolutionnaire, entreprise de moralisation précoce inspirée par la peur sociale, besoin de main-d'œuvre féminine consécutif à la Révolution industrielle, représentation valorisante des 2-6 ans chez des mères aisées et des médecins, demande des familles populaires. De même l'histoire des établissements secondaires laïcs aux XIX ${ }^{\mathrm{e}}$ et $\mathrm{XX}^{\mathrm{e}}$ siècles exige-t-elle de s'intéresser à d'autres sujets, comme le développement de l'État et ses relations avec l'Église romaine, l'évolution du pouvoir municipal et régional, les mutations de l'urbanisme, la formation des élites et la démocratisation de la société, l'encadrement des jeunes et leur culture ${ }^{17}$. Cet élargissement des perspectives incite à rechercher obsessionnellement les effets du temps ou, plus précisément, de l'enchevêtrement des durées cher à Fernand Braudel: temps spécifique de la doctrine ou de l'institution étudiée, conjonctures particulières de la société concernée, longue durée de l'outillage mental et des rapports des groupes sociaux avec leur milieu. C'est ainsi qu'au-delà de l'approche globale de «la» salle d'asile, cette devancière de l'école maternelle répandue à partir de 1828 , le chercheur de formation historienne se devait de traquer patiemment les traits permanents «des» institutions de jeunes enfants et les retombées des mutations économiques, sociales, politiques et culturelles sur leur fonctionnement et leur représentation.

Poussé par cette première ambition, l'historien de l'éducation peut être amené à traverser avec profit d'autres champs de recherche, relatifs à l'histoire de la famille, des enfants, des jeunes et du genre. «Un maillon dans une chaîne [...] entre la famille, dont il reçoit ses élèves, et la société, à laquelle il les destine» : cette définition du système scolaire comme objet d'histoire s'accompagnait d'une ouverture pionnière sur l'étude du "couple et l'enfant» et sur celle de "l'avènement des copains ${ }^{18}$. Si le détour par le rôle éducatif de la famille - lui-même objet de recherche autonome -

\footnotetext{
16. Luc 1997.

17. Luc 2005.

18. Prost 1981, 14.
} 
reste nécessaire pour certains sujets, l'histoire de l'enfance et de la jeunesse a suffisamment progressé pour être considérée aujourd'hui comme une source d'éclairage spécifique $^{19}$. C'est elle qui analyse les comportements et les représentations sociales de ces tranches d'âges, dont on sait qu'ils influencent les politiques et les pratiques éducatives. Pour la seule éducation scolaire, l'histoire des collèges et des lycées des années 1950 et 1960 a ainsi gagné à prendre en considération l'émancipation de l'adolescence et les inquiétudes suscitées par la «crise de la jeunesse». La même suggestion peut être faite à propos de l'histoire du genre. Il n'est plus possible aujourd'hui de négliger la construction des identités féminines et masculines ou les rapports de pouvoir entre les sexes dans l'approche historique des contenus et des méthodes d'enseignement, de la professionnalisation des personnels et des interactions parmi les éducateurs et les éduqués ou entre ces deux catégories ${ }^{20}$.

Propres à sa discipline ou empruntés à d'autres (sociologie, ethnologie, philosophie, psychologie, etc.), les paradigmes et les instruments à la disposition de l'historien de l'éducation sont trop nombreux pour être évoqués ici. «J'ai appris quelques rudiments de ces sciences humaines que dédaigne d'ordinaire le gros des historiens", constate l'historienne Françoise Mayeur, professeur d'histoire de l'éducation, pendant treize ans, à l'UFR de sciences de l'éducation de l'université de Lille III ${ }^{21}$. Il faut dire que les différences de langage jouent un rôle inhibiteur: l'abondance de termes techniques ou simplement abscons rebute des chercheurs habitués à une langue peu conceptualisée. Mais quand cet obstacle est minime ou levé, le détour mérite d'être effectué, comme il l'a souvent été: l'historien peut intégrer quelques nouveaux ingrédients dans son miel sans trahir son procédé de fabrication. L'ouverture sur d'autres sciences humaines et sociales lui permet d'enrichir ses questionnements et son argumentation, à condition de ne pas plaquer sur le passé des concepts importés et de dégager la particularité de chaque démarche. Là où l'approche strictement historienne des politiques d'éducation attache de l'importance aux acteurs, aux événements et aux prises de décisions (comme celles d'un Guizot en 1833 ou d'un Edgar Faure en 1968), la lecture sociologique, plus déterministe, souligne les pesanteurs du social, génératrices d'effets pervers, par exemple dans la mise en œuvre de la réforme Carcopino de $1941^{22}$.

Les curiosités et les outils de l'historien de l'éducation varient en fonction de l'évolution des sciences sociales en général et de sa discipline en particulier. L'érosion, à partir des années 1980, des modèles d'interprétation inspirés par Marx, Bourdieu ou Foucault réduit ou fait disparaître la lecture critique de l'histoire des structures éducatives en termes exclusifs de domination, de discrimination ou de normalisation. Inversement, l'audience parfois tardive de l'individualisme méthodologique de Raymond Boudon, de la microsociologie de l'interaction d'Erving Goffman ou de la

\footnotetext{
19. Luc 2008; Bantigny \& Baubérot 2008.

20. Rogers 2007.

21. Mayeur 1995, 35.

22. Prost 1992.
} 
théorie du capital humain popularisée par Carlo Cippola attire l'attention sur l'autonomie et la rationalité des individus et des familles, l'importance du face-à-face entre les personnes et la logique propre du marché scolaire. Au sein même de la recherche historique, le délaissement de la macro-histoire au profit de la micro-histoire contribue à revaloriser le local, l'individuel et le singulier comme facteurs explicatifs de la société. En histoire de l'éducation, ce recadrage a permis de découvrir les limites de la mise en œuvre ou de l'efficacité de certaines entreprises «disciplinaires", les résistances des publics à éduquer, la porosité des frontières entre l'école des notables et celle du peuple, les accommodements du modèle scolaire républicain avec les «petites patries» ou les spécificités du fonctionnement des établissements scolaires.

Sans nier le schématisme des anciennes analyses, ni l'intérêt des nouvelles, on peut se demander si le balancier n'est pas reparti trop loin dans l'autre sens. Depuis que les conduites individuelles et les processus de régulation ont été heureusement tirés de l'ombre, il est devenu de bon ton, çà et là, de négliger les tensions et les conflits collectifs qui agitent toute société. Pour échapper aux $a$ priori, il vaudrait mieux convoquer parfois quelques-uns des anciens concepts (contrôle social, discipline des corps, discrimination, habitus, champ social, etc.), et apprécier leur degré de pertinence sur pièces. La même remarque peut être faite à propos de l'approche statistique, passée de mode avec l'ambition quantitative associée aux problématiques et aux catégories de l'histoire sociale traditionnelle. A-t-elle été victime de ses seules insuffisances ou, aussi, des efforts exigés par la construction de séries cohérentes? Avec les précautions méthodologiques qui s'imposent, pourcentages, tris croisés, coefficients de corrélation ou analyses factorielles restent utiles si l'on ne veut pas s'en remettre totalement à la litanie des «beaucoup », « un peu», «rarement».

D'autres interrogations concernent l'attention, par ailleurs légitime, portée aux établissements et, plus largement, à l'autonomie des institutions éducatives et de leur culture. Si elle influençait trop l'histoire de l'éducation scolaire, cette approche pourrait enfermer la recherche entre les murs de l'école. Or l'expérience des années 19801990 - à savoir la remise en cause des interprétations structurelles du social, jusque-là dominantes - devrait inciter les chercheurs à ne pas adopter systématiquement les modèles en vogue à un moment donné. Peu médiatique, certes, la confrontation des objets et des perspectives n'en reste pas moins l'un des principes de base de la recherche. L'histoire des apprentissages de la lecture a progressé en s'intéressant aux usages sociaux de cet instrument. L'histoire des manuels a dépassé le simple inventaire critique des idéologies sous-jacentes en replaçant leurs messages dans le contexte politique et culturel de leur production. L'histoire de la classe et des interactions qui s'y produisent exige de prendre en compte l'histoire personnelle - donc en partie extrascolaire des élèves. L'histoire des pratiques pédagogiques et des contenus réellement enseignés, donc de la marge de manœuvre des enseignants, ne peut ignorer leurs origines, leur formation, leur carrière et leurs éventuels engagements, professionnels (par exemple, dans les mouvements d'éducation nouvelle) ou syndicaux. C'est dire que l'histoire de l'éducation ne doit pas renoncer à combiner, autant que possible, le contexte, les attentes des groupes sociaux et les logiques institutionnelles, nationales ou locales, avec les 
profils, les expériences subjectives et les pratiques individuelles des différents acteurs. La prosopographie des formateurs et des administrateurs reste d'ailleurs un chantier d'actualité si l'on considère les travaux achevés, entre 2004 et 2007, sur les recteurs, de 1808 à 1940, les directeurs de MJC au cours des années 1960 et les enseignants de breton dans les années 1970-1980, ou la grande enquête du Service d'histoire d'éducation sur les enseignants des établissements d'enseignement supérieur public, de 1808 à 1940. Le même raisonnement peut être appliqué à l'histoire des diverses strates des représentations : figuration des établissements, mise en scène des éducateurs et des éduqués, schèmes de leur perception du monde. Là encore, la pertinence de l'investigation n'exclut pas son prolongement: l'analyse complète d'une représentation exige de connaître le profil, le vécu et l'action de ceux qui la conçoivent, qui la reçoivent et, le cas échéant, qui en sont l'objet. Comme les autres champs de la discipline, l'histoire de l'éducation gagne à demeurer «sociale et culturelle, indissociablement ${ }^{23}$, donc à considérer les représentations comme des «formes qui sont toujours, simultanément, expressives et constructives du social ${ }^{24}$. Plusieurs des colloques organisés entre la fin de l'année 2006 et l'automne 2008 sur l'histoire de la seule éducation scolaire attestent d'ailleurs la diversité persistante de ses objets. Ils portent aussi bien sur les racines historiques du débat pédagogique, les plans d'éducation, la production des savoirs, la place de la religion à l'école et la certification des études secondaires, que sur l'État éducateur, les recteurs, l'identité féminine enseignante, les profils et les carrières des personnels du secondaire et du supérieur, les migrations des étudiants et leur place dans la société ${ }^{25}$.

Lorsque l'objet d'étude s'y prête, il est enfin utile de sortir de la bibliographie nationale pour découvrir d'autres approches et d'autres conclusions à propos de l'histoire de l'éducation dans d'autres pays ou en France même. Si la production anglo-

23. Prost 1997.

24. Kalifa 2005.

25. Étudiant-e-s en mouvements: internationalismes et internationales, cosmopolitismes et migrations étudiantes, 1870-200o (GERME - BIDC - Université de Reims, décembre 2006); Les stratégies de «l'État éducateur ". Études de cas et problématiques (Paris IV, mai 2007); L'enseignement secondaire féminin et l'identité féminine enseignante (IUFM de Champagne-Ardenne, juin 2007); Plans d'éducation et société en France et en Europe, de l'Ancien Régime à nos jours (IUFM d'Aquitaine et Bordeaux III, octobre 2007); L'État et les savoirs: institutionnalisations, résistances, alternatives, 1808 -milieu XIX ${ }^{e}$ siècle (Service d'histoire de l'éducation, SHE - IHRF, Paris I, octobre 2007); Sociohistoire des institutions scientifiques et éducatives (Nancy II, octobre 2007); Étudier ailleurs, étudier malgré tout. Migrations étudiantes et relations internationales. XVI siècle-1962 (Toulouse-le-Mirail, octobre 2007); Dieu à l'école: éducation et religion en Europe du Nord-Ouest et en Amérique du Nord de 1800 à nos jours (Université du Littoral, novembre 2007); Les hommes et les femmes de l'université: deux siècles d'archives (Archives de France, mars 2008); L'éducation entre héritages et modernité (Université de Rouen, Musée Oberlin, août 2008); Histoire sociale des enseignants du supérieur, XIX ${ }^{e}-X X^{e}$ siècles (SHE, automne 2008) et les manifestations co-organisées par Lille III, Paris IV et le SHE (INRP-ENS), qui ont choisi de travailler ensemble à l'occasion de la commémoration scientifique du bicentenaire de la naissance de l'université: L'État et l'éducation, 1808-2008 (La Sorbonne-ENS, mars 2008), Les recteurs d'académie et la fonction rectorale. Deux siècles d'histoire, 1808-2008 (La Sorbonne, mars 2008), Le baccalauréat et la certification des études secondaires: exception française ou pratique européenne? 1808-2008 (Lille III, juin 2008). 
saxonne attire à juste titre l'attention, par son volume, son accessibilité et l'originalité de certaines de ses perspectives (par exemple, à propos de l'histoire des universités), il ne faut pas oublier sa tendance, parfois, à «se constituer comme un en-soi», à l'écart des publications rédigées dans d'autres langues ${ }^{26}$. On a donc intérêt à consulter aussi la dynamique revue internationale Paedagogica historica, où les contributions européennes sont très nombreuses, et à se reporter, lorsque l'obstacle linguistique peut être levé, aux travaux allemands, italiens, espagnols, portugais ou brésiliens, pour ne citer qu'eux.

\section{L'histoire de l'éducation, observatoire d'une société}

Cet inventaire des territoires et des pratiques paraîtra disproportionné au regard de certaines contraintes de la recherche: lacunes de la documentation, disponibilité réduite des enseignants-chercheurs, durée limitée - un ou deux ans - de la préparation d'un master. Et le tableau reste incomplet, puisque rien n'y transparaît des tâtonnements imposés par la dispersion de la documentation, du désappointement provoqué par l'opacité persistante du passé, du bricolage créateur à partir des concepts et des sources ou de l'émotion du chercheur lorsque, selon la belle formule de Marc Bloch qui le compare à l'ogre de la légende, «il flaire la chair humaine [et] sait que là est son gibier».

En réalité, ce panorama propose plus un ensemble de repères qu'un programme impératif pour n'importe quel sujet, étudié dans n'importe quel cadre. Aujourd'hui comme hier, la préparation d'un doctorat ou d'un livre offre le cadre le plus propice à l'extension des curiosités. "Je voulais tenter une histoire aussi complète que possible: pédagogique, administrative, mais aussi sociale», explique Françoise Mayeur au début de l'exposé de soutenance de son doctorat ès lettres, L'enseignement secondaire des jeunes filles, 1867-1924, avant de détailler son itinéraire: «Il fallait donc décrire un système éducatif particulier, les intentions qui l'animaient, faire leur part aux élèves, aux professeurs, aux établissements et aux règlements qui les régissaient, puis prendre en compte l'évolution du tout durant près de cinquante ans ${ }^{27}$. Les articles, les communications à des colloques et les masters portent plutôt sur des objets ou des éclairages partiels, mais dont l'étude gagne toujours à s'inscrire dans une problématique élargie, voire dans les débats relatifs aux orientations de la discipline. Si l'on accepte cette démarche, l'histoire d'une réforme éducative, des contenus d'une catégorie de manuels ou des profils d'une population d'élèves devrait, au moins, poser les questions respectives des limites de l'application de toute décision gouvernementale, de l'usage effectif des livres de classe ou, inversement, de la construction et de la réception de l'image d'un établissement d'éducation.

26. Compère $1995,105$.

27. Mayeur 1976, 104. 
L'histoire de l'éducation doit sa richesse à la pluralité et à la transversalité de ses curiosités, qui en font l'un des carrefours de l'histoire d'une société. Elle étudie les deux dynamiques à l'œuvre dans la conception et le fonctionnement de toute entreprise éducative: une logique interne et des facteurs externes, propres à une population ou à une société particulière dans un espace et un temps donnés. Elle se prête bien à la variation des échelles d'observation, de l'analyse des doctrines pédagogiques, des plans d'éducation et des débats nationaux au décryptage des représentations et des pratiques individuelles. Elle associe des regards venus de disciplines différentes. En conservant cette ouverture, elle préservera, comme le recommandait Henri-Irénée Marrou, sa capacité à faire reconnaitre son objet comme « une condensation de la vie, de la civilisation, d'une société».

Jean-Noël LuC

Université Paris-Sorbonne - Paris IV

\section{Références bibliographiques ${ }^{28}$}

Bantigny L., Baubérot A. (2008), «Jeunes, jeunesse», in Dictionnaire d'histoire culturelle de la France contemporaine, C. Delporte, J.-Y. Mollier, J.-F. Sirinelli (dir.), Paris, PUF.

Caspard P. (1988), «Histoire et historiens de l'éducation en France», Les dossiers de l'éducation, $\mathrm{n}^{\circ} 14-15$, p. 9-29.

CASPARd P. (2006), Service d'histoire de l'éducation (INRP-ENS). Rapport scientifique 2004 et 2005, Lyon, INRP.

Caspard P. (2008), "Mémoire et histoire de l'éducation», in Dictionnaire de l'éducation, A. Van Zanten (dir.), Paris, PUF.

Charmasson T. (dir.) (2006), Histoire de l'enseignement, XIX ${ }^{e}-X X^{e}$ siècles. Guide du chercheur, Paris, INRP.

Chervel A. (1988), «L'histoire des disciplines scolaires. Réflexions sur un domaine de recherche», Histoire de l'éducation, n 38, p. 59-119.

Chervel A. (1998), La culture scolaire. Une approche historique, Paris, Belin.

Compère M.-M. (1995), L’histoire de l'éducation en Europe. Essai comparatif sur la façon dont elle s'écrit, Paris - Berne, INRP - Peter Lang.

Compère M.-M., Savoie P. (dir.) (2001), «L'établissement scolaire. Des collèges d'humanités à l'enseignement secondaire, $\mathrm{XVI}^{\mathrm{e}}-\mathrm{XX}^{\mathrm{e}}$ siècle», Histoire de l'éducation, n’ 90.

28. Pour ne pas allonger cette bibliographie, on y a surtout placé des études historiographiques. 
Compère M.-M., Savoie P. (2005), «L'histoire de l'école et de ce qu'on y apprend », Revue française de pédagogie, $\mathrm{n}^{\circ}$ 2, p. 107-146.

Debesse M., Mialaret G. (dir.) (1971-1972), Traité des sciences pédagogiques, Paris, PUF [t. II Histoire de la pédagogie; t. III Pédagogie comparée].

Havelange I. (2002), «Vingt ans de bibliographie d'histoire de l'éducation française (19791998) ", Histoire de l'éducation, n’ 93, p. 59-9o.

KALIFA D. (2005), «L'histoire culturelle contre l'histoire sociale? », in L'histoire culturelle du contemporain, L. Martin, S. Venayre (dir.), Paris, Nouveau Monde, p. 75-84.

LUC J.-N. (1997), L'invention du jeune enfant au XIX ${ }^{e}$ siècle. De la salle d'asile à l'école maternelle, Paris, Belin.

LUC J.-N. (2005), «À la recherche du "tout puissant empire du milieu”. L'histoire des lycées, du Second Empire au XXI" siècle», in Lycées, lycéens, lycéennes. Deux siècles d'histoire, P. Caspard, J.-N. Luc, P. Savoie (dir.), Paris, INRP, p. 11-56.

LUC J.-N. (2008), «Enfants, enfance», in Dictionnaire d'histoire culturelle de la France contemporaine, C. Delporte, J.-Y. Mollier, J.-F. Sirinelli (dir.), Paris, PUF.

Mayeur F. (1976), «L'enseignement secondaire des jeunes filles (1867-1924) », Mouvement social, n’96, p. 103-110.

Mayeur F. (1995), «Un itinéraire en histoire contemporaine», Bulletin de la société des amis de l'ENS, n' 197, p. 30-36.

Plaisance E., Vergnaud G. (1993), Les sciences de l'éducation, Paris, La Découverte.

Prost A. (1981), Histoire générale de l'enseignement et de l'éducation en France, t. IV : L'école et la famille dans une société en mutation, Paris, Nouvelle librairie de France.

Prost A. (1992), Éducation, société et politique. Une histoire de l'enseignement en France, de 1945 à nos jours, Paris, Seuil.

Prost A. (1997), "Sociale et culturelle, indissociablement», in Pour une histoire culturelle, J.-P. Rioux, J.-F. Sirinelli (dir.), Paris, Seuil.

Rogers R. (2007), «L'éducation des filles. Un siècle et demi d'historiographie», Histoire de l'éducation, n' 114-115.

Samaran C. (dir.) (1961), L’histoire et ses méthodes, Paris, Gallimard.

Sirinelli J.-F. (dir.) (1992), Histoire des droites en France, t. II : Cultures, Paris, Gallimard. 


\title{
L'HISTOIRE DE L'ÉDUCATION ET DE L'ENSEIGNEMENT DANS ET HORS LES SCIENCES DE L'ÉDUCATION
}

\begin{abstract}
Résumé: L'histoire de l'éducation et de l'enseignement, qui est familière dans les sciences de l'éducation, est une histoire-mémoire qui emprunte ses démarches et ses objets à l'histoire classique des idées. Elle a trois caractéristiques principales: 1) elle se centre sur des auteurs et des œuvres; 2) elle s'intéresse d'abord aux origines des doctrines et des institutions;3) elle prête une efficacité spéciale aux idées dans l'histoire. On pourra saisir sa différence avec l'histoire socioculturelle développée ces dernières années à l'extérieur des sciences de l'éducation, si l'on prend pour exemple les résultats obtenus par cette dernière sur le terrain de l'histoire des savoirs scolaires.
\end{abstract}

Mots clés: histoire de l'éducation et de l'enseignement, histoire des idées, histoire socioculturelle, histoire des savoirs scolaires.

\section{Diversité des approches}

D'abord un constat. Depuis longtemps une histoire traditionnelle est pour l'essentiel (je ne dis pas en totalité) en circulation dans les sciences de l'éducation. C'est une histoire constituée sur un enjeu patrimonial car elle a pour fonction de produire et de conserver la mémoire collective des enseignants et de l'institution scolaire ellemême. Cette histoire quasi «identitaire » était jadis fort prisée dans les Écoles normales primaires, notamment sous la Troisième République; et elle est très bien exposée, par exemple, au Musée national de l'éducation de Rouen, comme dans de nombreux musées locaux, qui fleurissent ici ou là ces dernières années...

L'histoire traditionnelle (ce dernier qualificatif étant utilisé ici sans aucune nuance dépréciative) de l'éducation et de l'enseignement a des objets de prédilection qui dessinent deux domaines d'intérêt principaux: d'une part les doctrines éducatives et d'autre part les institutions d'enseignement - donc les réformes qui les créent, les débats qui les suscitent, les lois qui les définissent, etc.

L'histoire des doctrines s'est constituée au XIX ${ }^{\mathrm{e}}$ siècle, en retard sur l'Allemagne d'ailleurs, avec l'Histoire critique des doctrines de l'éducation en France, de G. Compayré ${ }^{1}$, puis avec le Dictionnaire de pédagogie et d'instruction primaire, de

1. Compayré 1879 . 
F. Buisson ${ }^{2}$, qui comporte de nombreuses notices à finalité historique - dont certaines sont devenues des sources très précieuses même quand les sources primitives sont occultées. Je citerai aussi l'ouvrage moins connu de J. Paroz, Histoire universelle de la pédagogie ${ }^{3}$, peut-être le premier du genre (sans omettre de signaler dans ce volume une savoureuse visite au Philanthropin de Basedow, à Dessau). On peut lire aussi, plus proche de nous, le livre de J. Leif et G. Rustin, Pédagogie générale par l'étude des doctrines pédagogiques ${ }^{4}$.

Une telle histoire des doctrines comporte des rubriques obligées qui nous sont très familières. On y rencontre souvent des auteurs et des œuvres mémorables: voici les «grands pédagogues» et leurs ouvrages canoniques. Mais on y découvre aussi des "courants » - c'est-à-dire des pensées unifiées et propres à un milieu, à un groupe, un pays, une époque ou autre. On pense au livre de L. Legrand sur L'influence du positivisme dans l'ouvre scolaire de Jules Ferry ${ }^{5}$; ou bien à la plus récente Histoire de la pédagogie du XVII siècle à nos jours ${ }^{6}$, dirigée par G. Avanzini - au moins pour un certain nombre des contributions. Et sans doute est-il légitime d'associer à ces références certains travaux actuels qui, toujours dans le cadre des sciences de l'éducation, ont une facture moins classique et constituent des apports notables pour nos réflexions. C'est le cas d'un ouvrage réalisé par l'équipe de Paris VIII et A. Savoye (qui se réclament de la «socio-histoire»), sur L'Éducation nouvelle, histoire, présence, ave$n i r^{7}$. Dans la même veine, l'ouvrage dirigé par D. Denis et P. Kahn, sur le Dictionnaire de F. Buisson ${ }^{8}$, a permis de resaisir sinon une doctrine républicaine de l'école et de la pédagogie, du moins un «moment» intellectuel propre à l'élaboration républicaine de l'école.

Le second domaine auquel j'ai fait allusion est l'histoire des institutions, laquelle s'associe le plus souvent à l'histoire des acteurs qui font vivre et évoluer de telles institutions. On pourrait se reporter là encore à des ouvrages de la génération précédente comme l'Histoire des institutions scolaires, de J. Leif et G. Rustin ${ }^{9}$. Plus avant, l'historiographie dite républicaine n'a pas été en reste, pour des raisons politiques que chacun comprendra. Viennent à l'esprit les deux volumes d'A. Léaud et E. Glay, L'école primaire en France ${ }^{10}$. C'est une voie qu'a suivie plus récemment, et avec bonheur, C. Lelièvre ${ }^{11}$.

\footnotetext{
2. Buisson 1878-1887, seconde édition en 1911.

3. Paroz 1868.

4. Leif \& Rustin 1953.

5. Legrand 1961.

6. Avanzini 1981.

7. Ohayon et al. 2004.

8. Denis \& Kahn 2003.

9. Leif \& Rustin 1954.

10. Léaud \& Glay 1934.

11. Lelièvre 1990.
} 
Évidemment, cette histoire s'appuie sur, ou coexiste davantage que la précédente variante avec les travaux d'historiens qui n'appartiennent pas directement aux sciences de l'éducation, mais y ont des relations et des intérêts avérés. A. Prost est en tête de ces historiens, bien sûr, depuis son ouvrage de 1968 (L'enseignement en France, 1800-1967 $7^{12}$ ), toujours à lire, même si l'entreprise mériterait d'être réactualisée par les connaissances d'aujourd'hui, si développées. Les spécialistes savent en outre l'impressionnant apport d'auteurs plus anciens, comme M. Gontard ou F. Ponteil, etc.

Ces repères étant posés pour indiquer des lignes de force sans souci d'exhaustivité, je voudrais interroger les particularités de la configuration évoquée. Pour ce faire, il est commode - et plausible - de distinguer cette histoire traditionnelle et patrimoniale de l'histoire plus scientifique qui se pratique ou est censée se pratiquer dans ce que F. Furet appelait l'atelier de l'histoire, et qui a fourni ces dernières années des données originales sur différents sujets d'éducation et d'enseignement. Je me garde de hiérarchiser ou d'opposer les deux genres. Je ne suis pas là, et pas qualifié d'ailleurs, pour décerner des bons et mauvais points. Bien faite, l'histoire traditionnelle est toujours utile; je veux juste proposer des définitions de ses objets et plus encore de ses problèmes.

Pour saisir les progrès de la connaissance historique hors du cadre strict des sciences de l'éducation, on peut consulter la note de synthèse publiée par M.-M. Compère et $\mathrm{P}$. Savoie dans la Revue française de pédagogie ${ }^{13}$. S' il ne fallait mentionner qu'une ou deux références depuis trente ans, ce seraient sans doute le très important travail de F. Furet et J. Ozouf sur l'alphabétisation des Français ${ }^{14}$, et celui de R. Chartier, M.-M. Compère et D. Julia sur l'éducation du XVI ${ }^{e}$ au XVIII ${ }^{e}$ siècle ${ }^{15}$. Ces deux ouvrages, parmi tant d'autres, se rejoignent dans leur traitement des phénomènes de l'alphabétisation et de la scolarisation - élémentaire ou dans les collèges d'Ancien Régime. Ils observent des phénomènes sociaux et culturels, comme l'histoire des mentalités d'ailleurs, dans le sillage de l'histoire économique et sociale de l'école des Annales. Quelles différences peut-on alors repérer entre cette approche et celle que j'ai qualifiée de patrimoniale (si, encore une fois, on accepte cette dualité trop simplificatrice sans doute)?

L'histoire traditionnelle de l'éducation, comme histoire des doctrines et des institutions éducatives, est avant tout une histoire des idées; ou du moins c'est dans l'histoire des idées qu'elle trouve ses enjeux majeurs. L'approche est classique, elle vient du XIX ${ }^{e}$ siècle et de l'histoire littéraire (qui s'est imposée comme discipline dans les lycées). En s'attachant aux auteurs et aux œuvres, elle privilégie en outre les discours fortement valorisés, ceux auxquels on accorde un grand crédit de cohérence et de rationalité, contrairement à d'autres qui ne sont visibles que dans les profondeurs de la mémoire et de l'archive.

\footnotetext{
12. Prost 1968.

13. Compère \& Savoie 2005.

14. Furet \& Ozouf 1977.

15. Chartier et al. 1976.
} 


\section{Le primat des auteurs}

À ce titre, il s'agit d'abord, comme je le suggérais plus haut, d'une histoire peuplée de personnalités, d'auteurs, plus exactement de «grands auteurs». Telles sont justement les références obligées - de Montaigne à Freinet, ou Piaget..., en passant par beaucoup d'autres, avec des «innovateurs», comme Jean-Baptiste de La Salle ou Pestalozzi, des «réformateurs», comme Jules Simon ou Ferdinand Buisson, etc. Le besoin d'invoquer ces figures admirables - souvent mythifiées - explique que cette histoire s'achève en galerie de portraits. L'important est de savoir qui a produit telle doctrine, qui est le promoteur de tel courant, le fondateur de telle institution ou l'inventeur de telle méthode. Ces questions d'appartenance remplissent la fonction patrimoniale et le besoin identitaire dont je parlais plus haut. Ceci suppose que les auteurs se définissent par des identités, des formes de conscience et, en quelque manière, des intentions, claires ou obscures, qui se lisent dans leurs œuvres et se retrouvent dans les courants de pensées qu'animent des disciples ou des adeptes. Les frontières qui séparent les doctrines prennent alors une grande importance : elles réservent telles «idées » à telle doctrine et non à telle autre, elles mettent les auteurs et les courants, soit à distance, soit en conflit les uns avec les autres. Républicains contre catholiques, laïcs contre cléricaux, méthodes nouvelles contre routines rétrogrades, etc.: toutes ces dualités et d'autres du même type, fermement maintenues, concentrent le pouvoir d'explication de l'histoire-mémoire de l'éducation.

L'histoire socioculturelle par contre connait peu de héros. Comme elle objective de préférence des populations ou des masses, elle convoque des anonymes et des sans-grade, dont elle cherche à décrire les représentations et les comportements collectifs. Or il est entendu que ceux-ci ne peuvent être référés à des identités et des intentions permanentes, qui seraient constitutives des pensées et des décisions qui ont fait date. Quand J.-C. Caron examine le traitement de la violence dans les contextes éducatifs du XIX ${ }^{e}$ siècle $^{16}$, quand J.-F. Chanet analyse la production des normes culturelles de l'école primaire sous la Troisième République ${ }^{17}$, et quand J.-N. Luc décrit la naissance de l'école maternelle dans le cadre plus général du souci pour la petite enfance ${ }^{18}$, ils rendent compte aussi bien des pensées assurées que des jugements hésitants, des projets durables que des initiatives passagères et confuses. Ce faisant, ils atteignent des formes de conscience plus labiles et des modes d'expérience moins élaborés, moins établis dans des frontières doctrinales, et qui se réfèrent peu à des conceptions exposées dans une littérature et diffusées dans des programmes.

\footnotetext{
16. Caron 1999.

17. Chanet 1996.

18. Luc 1997.
} 


\section{La recherche des origines}

L'histoire traditionnelle porte ensuite une attention soutenue aux moments d'origine - origines des doctrines autant que des institutions. Elle se demande quel auteur, quelle œuvre, ou quelle initiative se trouvent au début d'une tradition et se retrouvent ensuite dans les autres œuvres, les autres initiatives, celles qui, après ce point de création radicale, reçoivent un héritage et sont prises dans une filiation. Cette interrogation en appelle presque toujours une autre, pour savoir quel auteur ou quelle idée d'un auteur exerce, depuis ce moment d'origine, une influence sur les conceptions ou les initiatives des successeurs, individus ou groupes particuliers, aux différentes époques. Origine, filiation, influence, telles sont les trois grandes catégories organisatrices de l'histoire patrimoniale. Rappelons qu'on doit à Foucault une analyse critique décisive de ces notions dans son ouvrage épistémologique de 1970, L'archéologie du savoir ${ }^{19}$.

La primauté accordée aux questions d'origine oriente le choix des sources ainsi que la manière de périodiser les phénomènes. L'histoire traditionnelle s'accorde à une chronologie événementielle, le plus souvent politique. Elle se situe par exemple " de Guizot à J. Ferry », elle se cadre dans un «moment républicain », etc. (ce qui renforce la solidarité de l'histoire des idées et de l'histoire des institutions). Le tropisme de l'origine donne du poids aux événements, qu'ils soient politiques, sociaux, voire culturels...; étant entendu que l'événement est producteur d'une temporalité continue, celle qu'il a ouverte et qui, en quelque sorte, ne sera pensable que par lui.

D'après ce qui précède, on peut se demander si le succès, dans les sciences de l'éducation, de la théorie de la «forme scolaire» de G. Vincent, au moins sa première version, de 1981 ( «forme scolaire de socialisation » d'après l'expression primitive de l'auteur), ne tient pas à ce qu'elle fait écho à cette double prédilection de l'histoire des idées - l'avantage donné aux fondateurs et la sensibilité à l'origine. Cette théorie semble en effet très adéquate à ces passions de l'histoire patrimoniale: les fondateurs, ce sont en l'occurrence Jean-Baptiste de La Salle et les frères des écoles chrétiennes, et l'origine, c'est l'enseignement simultané (le mode de relation impersonnel qui exige le respect des règles et non plus la soumission à la personne du maître). Cet enseignement, en effet, constitué et diffusé comme une doctrine spéciale, fera sentir ses effets sur les innovations ultérieures.

Les orientations et démarches classiques de l'histoire des idées ont suscité de nombreuses questions, théoriques, méthodologiques, historiographiques. Les discussions ne manquent pas, depuis la première génération de l'école des Annales et ceux qui, comme Febvre, appelaient de leurs vœux une histoire intellectuelle resituée dans le cadre d'une histoire sociale (voir son ouvrage pionnier sur Rabelais), ou plus tard quand l'histoire des mentalités a impulsé des travaux aussi marquants que ceux de R. Mandrou sur la culture populaire aux XVII et XVIII e siècles, ou de P. Ariès sur

19. Foucault 1970. 
l'enfance et la famille sous l'Ancien Régime. Il est d'ailleurs intéressant de savoir que Foucault, que je citais plus haut, adresse des reproches croisés à l'histoire des idées classique et à l'histoire des mentalités, qui s'en est pourtant affranchie.

Puisqu'on se détourne des auteurs et de leurs identités au profit des masses et des groupes, on traite avant tout des séries objectives et quantifiables de données, donc des séries qui, souvent, s'inscrivent dans des durées plus longues, sans se fixer sur des origines et des événements significatifs. C'est ainsi que Furet et Ozouf, en s'appuyant sur les relevés des signatures des actes de mariage, retracent les progrès de l'alphabétisation depuis le XVII ${ }^{\mathrm{e}}$ siècle, et suivent l'évolution des taux de population acculturée à la langue écrite ${ }^{20}$ aux différentes périodes, jusqu'au XIX ${ }^{\mathrm{e}}$ siècle. Et dès lors que l'alphabétisation n'est pas un processus tout à fait superposable à la scolarisation, on doit admettre que les institutions scolaires et les pensées pédagogiques suivent davantage qu'elles ne provoquent les mutations en cours, ce qui engage bien une tout autre manière d'en analyser la formation et d'en périodiser les développements ${ }^{21}$.

\section{L'efficacité des doctrines et de leur message}

L'histoire traditionnelle de l'éducation et de l'enseignement postule une capacité essentielle du message déposé dans les doctrines à susciter des projets et des décisions, à informer des pratiques, donc à engendrer l'histoire réelle, de telle sorte que celle-ci trouve en lui sa propre vérité. Ainsi voit-on l'histoire de la pédagogie vérifier les progrès de l'amour des enfants, l'histoire des institutions scolaires enregistrer la politique républicaine, ou l'histoire de la pédagogie s'achever avec les méthodes modernes. Il y a du sens dans l'histoire et un sens de l'histoire. Le présent explique le passé, et non l'inverse.

En outre, le point de vue de l'efficacité doctrinale (donc de l'unité du message) implique que l'on s'attache à saisir les thèmes qui, en déclinant les doctrines et leurs idées, les convertissent en significations, principes et règles disponibles pour des commentaires et des applications. On songe au thème de l' «enfant au centre " (principe puérocentrique), au thème de l' «élève actif» (norme psychologique), au thème de la

20. En fait, ils traitent aussi des données déjà recueillies un siècle avant eux par l'inspecteur d'académie Maggiolo, qui s'était vu confier une mission par le ministère de l'Instruction publique, et avait demandé à plus de 15000 instituteurs dans un grand nombre de départements d'aller dépouiller les actes d'état civil de leur commune (actes paroissiaux avant la Révolution), et de faire des relevés sur les signatures.

21. Rappelons les résultats très connus de ces travaux: une partie des Français sait lire depuis longtemps à l'époque de J. Ferry (sous Louis XIV, $1 / 3$ des Français et $1 / 7^{\mathrm{e}}$ des Françaises savent lire; puis, avant la Révolution, un homme sur deux et une femme sur quatre); longtemps, les femmes et les hommes ne sont pas entraînés au même titre dans ce courant d'acculturation (chez les femmes domine le savoirlire seulement); et le constat d'une France divisée en deux parties, l'une, bien alphabétisée, au nord de la ligne Saint-Malo - Genève, et l'autre, mal alphabétisée, en retard, plus lente, au sud de cette ligne, ce constat, donc, déjà effectué par l'inspecteur Maggiolo sous la Troisième République, était juste mais méritait d'être nuancé par toutes sortes de données géographiques, sociales, économiques. 
«laïcité» (idéal républicain), etc. Ainsi seraient éclairées, au-delà des doctrines et des œuvres, les activités des agents, habités par les fins que les doctrines prescrivent.

L'histoire sociale et culturelle de l'éducation n'a pas les mêmes visées. En constituant des séries, d'une part, elle cherche, on l'a vu, à objectiver sans préalable des comportements collectifs et des représentations; mais d'autre part, elle relie ces données de conduite et de mentalité non pas à l'unité de doctrines quelconques, mais à la pluralité des contextes sociaux et culturels à l'intérieur desquels elles sont visibles. C'est ainsi que les progrès de l'alphabétisation décrits par Furet et Ozouf s'expliquent par les évolutions anthropologiques et démographiques (les transformations de la famille), les évolutions économiques et sociales (les rapports de la ville et de la campagne, les différents types des exploitations rurales), les évolutions culturelles (l'expansion et la circulation de la culture écrite), etc. Les travaux de R. Chartier sur l'histoire des pratiques culturelles, et notamment des pratiques de lecture et des usages de l'imprimé, adoptent une démarche similaire, en interrogeant les supports (s'agit-il de littérature savante ou de livres de colportage?), les usages (lecture silencieuse ou oralisée?), les modes d'appropriation (en public ou dans l'intimité?), etc. Si bien qu'apparaissent deux grands types de lecture aux XVIII ${ }^{\mathrm{e}}$ et XIX ${ }^{\mathrm{e}}$ siècles. D'une part, une lecture «intensive» (traditional literacy), faite de révérence et de respect, qui confronte à un petit nombre de textes comme la Bible, des livres de piété, des almanachs, et qui privilégie des situations collectives (on lit et on écoute en société, dans la famille, à l'Église). D'autre part, une lecture « extensive», qui se développe de 1750 à 1850, exercée sur des textes plus nombreux, lus silencieusement et abordés dans une situation d'intimité, en s'entourant même des conditions matérielles appropriées, y compris un mobilier spécifique: bref une lecture laïcisée ${ }^{22}$.

En résumé, l'histoire traditionnelle de l'éducation et de l'enseignement reconstitue des espaces de pensée et d'action homogènes, qui connaissent, non pas des ruptures, mais des oppositions doctrinales (comme entre le respect de l'enfance et l'inculcation autoritaire, ou entre le dogmatisme chrétien et la tolérance républicaine). L'histoire socioculturelle au contraire traite des univers de discours et de pratiques hétérogènes, qui sont travaillés par des ruptures, des décalages, des déviations (comme entre l'alphabétisation des hommes et celle des femmes, ou entre des transmissions religieuses et des sociabilités profanes). Et du coup, alors que la première imagine les progrès d'une rationalité universelle où le présent joue toujours contre le passé, qu'elle rejette dans l'oubli, la seconde interroge certes des transformations irréversibles, mais aussi des adaptations précaires ou pérennes, réussies ou ratées, qui arrangent, entre le nouveau et l'ancien, des compromis originaux, évolutifs et complexes.

22. Voir notamment Chartier 1985, $88 \mathrm{sq}$. R. Chartier utilise des témoignages, des traces, prélevées dans les biographies, les romans et toutes sortes de récits, comme des journaux intimes, avec des descriptions, parfois incidentes et accessoires, des manières de lire. Importants également sont pour lui les documents iconographiques: tableaux, gravures, estampes. Voir aussi Chartier 1987. 


\section{Une histoire des savoirs scolaires}

Entre l'histoire patrimoniale et l'histoire socioculturelle, il y a au total une différence d'objets, et pas seulement de regards sur les mêmes objets. J'ai fait allusion à l'histoire de l'alphabétisation et de la scolarisation, mais je voudrais évoquer l'histoire de la culture scolaire, ainsi que celle, très proche, des pratiques et des techniques d'enseignement. Je voudrais donc compléter mon tour d'horizon en m'arrêtant aux contenus de l'enseignement. Aujourd'hui bien installée, une telle histoire nous révèle la formation des catégories de la culture scolaire et des disciplines scolaires. J'insiste sur ce point. L'attention aux contenus a été très faible dans le cadre de l'histoire-mémoire. Omission d'autant plus étonnante que le livre inaugural de Durkheim, L'évolution pédagogique en France ${ }^{23}$ (peut-être le livre dont les sciences de l'éducation doivent se réclamer avant tout autre) s'était donné cet objet précisément. L'indifférence a commencé d'être réparée à partir des travaux d'A. Chervel et d'un article de la revue d'Histoire de l'éducation en $1988^{24}$. Le même numéro comportait du reste un autre article très intéressant d'Hébrard, qui fixait un programme un peu différent. Il n'est pas inutile de rappeler que, dans les mêmes années, la sociologie anglaise du curriculum commence d'être bien connue en France.

Pour faire entrevoir les résultats de ce genre d'approche, je prendrai cette fois l'exemple des travaux d'A.-M. Chartier sur l'apprentissage scolaire de la lecture (voir notamment «Des abécédaires aux méthodes de lecture: genèse du manuel moderne avant les lois Ferry ${ }^{25}$ ). L'exemple est d'autant plus éclairant qu'on peut l'opposer à un autre, qui relève justement d'une histoire patrimoniale, l'étude publiée sous la plume de J. Guion dans l'ouvrage collectif de G. Avanzini que j'ai cité plus haut. D'après cet auteur, l'histoire de la lecture est en effet une suite de «méthodes » basée sur un principe général, après quoi, par inventions accumulées (la nouvelle épellation après l'ancienne, puis la syllabisation après l'épellation, etc.), on assisterait à un progrès nécessaire de la raison didactique, jusqu'à ce que s'affirment les techniques enfin rationnelles et efficaces. L'histoire, une fois de plus, se structure dans l'opposition entre les valeurs - les stéréotypes - du «rétrograde» et du «moderne».

Considérons cependant, avec A.-M. Chartier, le procédé de l'épellation en vigueur sous l'Ancien Régime et utilisé jusqu'au XIX ${ }^{\mathrm{e}}$ siècle. L'épellation de chaque mot ou syllabe est exigée des élèves avant toute lecture de mot. Comment comprendre le passage de l'ancienne épellation (bé, cé, dé) à la nouvelle ( $\mathrm{Be}, \mathrm{Ce}, \mathrm{De})$, étant entendu que la première se donne comme une manière de nommer les lettres alors que la seconde survient pour les prononcer, en rendant mieux la valeur phonique des consonnes dans la syllabe? Pour obtenir une réponse qu'on trouvera peu dans les justifications des pédagogues, s'il y en a, on doit faire deux constats. Le premier,

\footnotetext{
23. Durkheim 1938.

24. Chervel 1988.

25. Chartier 2005.
} 
d'une grande importance, c'est que cette variation va s'accorder à un apprentissage en langue française et non plus en latin. Le second, c'est que l'ancienne épellation s'exerce sur des textes transmis et appris oralement, des prières avant tout, donc des textes entendus et récités par cœur avant d'être déchiffrés, une fois les bases alphabétiques transmises; en revanche, la nouvelle épellation, apparue dès le XVIII ${ }^{\mathrm{e}}$ siècle, s'emploie dans des exercices où l'on mémorise des mots ou des syllabes qu'on réinvestit ensuite dans le déchiffrage de textes inconnus - provenant toutefois du même univers de culture religieuse. Que suggèrent donc ces constats? Que c'est d'abord dans le contexte d'une nouvelle culture que s'opèrent ces mutations, non sous l'influence d'un principe explicite et rationnel qu'une doctrine et qu'un auteur (mais lesquels et quand?) auraient réfléchi et transmis à des successeurs.

Lorsqu'ensuite, vers le milieu du XIX ${ }^{\mathrm{e}}$ siècle, les méthodes «sans épellation » préconisent la lecture directe des syllabes et des mots, et que se répandent la syllabisation et l'étude des correspondances graphophonétiques, les enjeux se déplacent encore. Prenons, en suivant là encore A.-M. Chartier, un livre comme l'Alphabet et premier livre de lecture, diffusé et soutenu par les autorités en 1832, au moment où le terme de «méthode» est vulgarisé, et qu'apparaît un vocabulaire nouveau comportant des termes étranges comme «citolégie», "statilégie», etc. Dans ce manuel, assez typique du courant moderne, on ne trouve plus l'ordre qui mettait en tête l'alphabet, qui débutait la lecture par des exercices sur les voyelles, qui continuait par des exercices sur les consonnes, qui se poursuivait par l'approche des syllabes en allant des plus simples aux plus complexes, etc. En fait, les lettres, qui sont désormais appariées à des familles de sons, sont présentées dans un ordre nouveau et, alors que le temps consacré aux syllabes augmente, les textes à découvrir, inconnus, arrivent plus tardivement dans le cursus. Or, ces changements, comme les précédents, s'expliquent en fonction de contextes qui privilégient cette fois une culture savante ou du moins une culture en voie de sécularisation - quoique non exempte d'intentions moralisatrices. Les élèves doivent toujours apprendre par cœur, mais les éléments et les contenus qu'ils mémorisent sont d'une autre nature, ils peuvent être moins religieux, plus « usuels », etc. C'est dans cette configuration où s'imposent à la fois le français et les visées d'une culture laïcisée, qu'apparaît la finalité suprême de l'orthographe; et, pour longtemps, le couple dictée-questions commence de régner sur l'école élémentaire.

Pour que le paysage soit complet, il faudrait saisir la convergence, très frappante, de ces évolutions avec d'autres, qui surviennent à l'interne cette fois, dans le cadre scolaire et pédagogique. D’une part, la mémorisation des éléments à prononcer est soutenue et quasi naturalisée par la simultanéité des apprentissages de la lecture et de l'écriture, introduite au début du XIX ${ }^{\mathrm{e}}$ siècle (spécialement par les écoles mutuelles). Et d'autre part, quelques dizaines d'années plus tard, autour de 1850, l'adoption très rapide des plumes métalliques permet de systématiser cette norme de la lecture et de l'écriture conjointes, par la facilité d'exécution et le gain de temps que procure cet outil (ne fût-ce que la taille des plumes d'oie occupant les débuts de la matinée de classe). Quand le système global sera en place, dans la seconde moitié du siècle et surtout sous la Troisième République, les manuels prescriront en même temps ce 
qu'on lit et ce qu'il faut écrire; ce sera le cas de la méthode Schuler, tant vantée par J. Guillaume dans le Dictionnaire de pédagogie et d'instruction primaire de Buisson.

On aura aperçu, je l'espère, le pouvoir d'explication de telles enquêtes qui prennent pour objet les rapports entre les pratiques scolaires et les contextes extérieurs. Il resterait bien sûr à comprendre comment ces rapports agissent dans la réalité de l'enseignement. Disons qu'à certaines conditions, les savoirs (scolaires) ne se séparent pas des usages culturels (non scolaires), ni des expériences que procurent et que commandent ces usages émanés d'une culture singulière. Il ne s'agit pas là de transfert de formes pédagogiques ou de transposition de contenus. À travers les usages se diffusent des idéaux et s'affirment des valeurs, donc se répandent des convictions qui attribuent à ces usages un statut d'évidence dans l'univers éducatif, leur donnant par là même une force de production et d'imposition de normes (la déduction des choix pédagogiques à partir des idéaux de culture et d'éducation caractérise la méthode de Durkheim dans L'évolution pédagogique en France). Le récit historique peut dès lors s'attacher à décrire la construction des normes de culture qui informent les savoirs scolaires - ce qui ne va pas sans adaptations de toutes sortes, car les normes sont des prescriptions toujours transformées par les agents qui les acceptent et les institutions où elles s'enracinent.

Cette approche a plusieurs mérites et pour finir je voudrais souligner celui qui apparait le plus clairement. C'est le nouveau regard sur les pratiques d'enseignement qu'elle a suscité. On peut même dire qu'une dimension importante des pratiques d'enseignement est devenue visible à partir du moment où on s'est intéressé aux savoirs scolaires dans leurs rapports avec les contextes socioculturels externes. Là réside sans doute la différence la plus importante avec l'histoire-mémoire qui se centre non pas sur la production des normes par en bas (depuis les contextes pratiques), mais sur l'application de règles par en haut (depuis les discours et les doctrines).

François JACQUET-FrANCILLON Université Charles de Gaulle - Lille III

\section{Références bibliographiques}

Avanzini G. (dir.) (1981), Histoire de la pédagogie du XVII siècle à nos jours, Toulouse, Privat.

Buisson F. (1878-1887, 1911), Dictionnaire de pédagogie et d'instruction primaire, Paris, Hachette.

Caron J.-C. (1999), À l'école de la violence. Châtiments et sévices dans l'institution scolaire au XIX siècle, Paris, Aubier. 
Chanet J.-F. (1996), L'école républicaine et les petites patries, Paris, Aubier.

Chartier A.-M. (2005), «Des abécédaires aux méthodes de lecture: genèse du manuel moderne avant les lois Ferry", in Histoires de lecture XIX ${ }^{e}-X X^{e}$ siècles, J.-Y. Mollier (éd.), Bernay, Société d'histoire de la lecture, p. 78-102.

Chartier R. (1985), «Du livre au lire», in Pratiques de la lecture, R. Chartier (dir.), Paris, Payot.

Chartier R. (1987), Lecteurs et lectures dans la France d'Ancien Régime, Paris, Seuil.

Chartier R., Compère M.-M., Julia D. (1976), L'éducation en France du XVI ${ }^{e}$ au XVIII ${ }^{e}$ siècle, Paris, SEDES.

Chervel A. (1988), « L'histoire des disciplines scolaires: réflexions sur un domaine de recherche», Histoire de l'éducation, n’ 38, p. 59-119.

Compayré G. (1879), Histoire critique des doctrines de l'éducation en France depuis le $X V I^{e}$ siècle, Paris, Hachette.

Compère M.-M., SAvoie P. (2005), «L'histoire de l'école et de ce que l'on y apprend », Revue française de pédagogie, $\mathrm{n}^{\circ} 152$.

Denis D., Kahn P. (dir.) (2003), L'école républicaine et la question des savoirs. Enquête au coeur du Dictionnaire de pédagogie de Ferdinand Buisson, Paris, CNRS.

Durkheim É. (1938), L'évolution pédagogique en France, Paris, PUF.

Foucault M. (1970), L’archéologie du savoir, Paris, Gallimard.

Furet F., Ozouf J. (1977), Lire et écrire. L'alphabétisation des Français de Luther à Jules Ferry, Paris, Minuit, 2 vol.

LÉAud A., Glay É. (1934), L'école primaire en France. Ses origines, ses différents aspects au cours des siècles, ses luttes, ses victoires, sa mission dans la démocratie, Paris, La Cité française, 2 vol.

LEGRAND L. (1961), L'influence du positivisme dans l'œuvre scolaire de Jules Ferry, Paris, Marcel Rivière.

Leif J., Rustin G. (1953), Pédagogie générale par l'étude des doctrines pédagogiques, Paris, Delagrave.

LeIF J., Rustin G. (1954), Histoire des institutions scolaires, Paris, Delagrave.

LeLIÈvRe C. (1990), Histoire des institutions scolaires (1789-1989), Paris, Nathan.

Luc J.-N. (1997), L'invention du jeune enfant au XIX siècle. De la salle d'asile à l'école maternelle, Paris, Belin.

Ohayon A., Ottavi D., Savoye A. (coord.) (2004), L'Éducation nouvelle, histoire, présence et devenir, Berne, Peter Lang.

PAROz J. (1868), Histoire universelle de la pédagogie, Paris, Delagrave.

Prost A. (1968), L'enseignement en France, 1800-1967, Paris, Colin. 



\section{POUR UNE ÉPISTÉMOLOGIE DE L'HISTOIRE DES IDÉES PÉDAGOGIQUES}

Résumé: La mémoire est devenue indépendante du sujet ou de la communauté qui en était dépositaire. La science occidentale moderne a fondé pour une large part sa légitimité sur l'éviction du sujet. L'exploration de l'entre-deux pédagogique vise au contraire à réduire cette dichotomie entre les traces et les sujets qui les ont générées, et inscrit l'«histoire des idées pédagogiques» dans au moins deux univers théoriques différents, la connaissance historique et l'herméneutique du sujet. L'épistémologie de la recherche en histoire des idées pédagogiques développée au long de cet article contribue à définir une forme possible pour un type de temporalité que Ricœur nomme «le temps du sens » : entre l'instance des sédimentations successives des dépôts d'archives et une démarche de distanciation critique.

Mots clés: pédagogie, histoire, histoire des idées, herméneutique, épistémologie.

Pour l'histoire, en tant que référentiel théorique, l'éducation et ses figures se présentent comme un référent ordinaire, analysé dans le cadre habituel de périodisations faisant autorité : antique ${ }^{1}$, ancienne, moderne, contemporaine... D'un point de vue tant méthodologique qu'épistémologique, l'historien s'intéresse à l'éducation comme il traite de l'art, de la guerre ou de la mode. Daniel Hameline affirme à ce propos que la confection des "grandes figures» répond dans ce cadre «à deux impératifs incompressibles: mettre en mémoire et faire mémoire $»^{2}$.

\section{À la recherche du temps perdu}

L'épistémologie de la recherche développée au long de cette communication contribue à définir l'émergence, au sein des sciences de l'éducation, d'une histoire des idées pédagogiques, semblable et différente de son aînée, forme possible, pour les pédagogues, d'un type de temporalité que Ricœur nomme «le temps du sens».

1. Marrou 1948.

2. Hameline 2001, XVI. 
Si l'on accepte comme une évidence que toute pensée, et par conséquent toute idée est, au moins à l'origine, incarnée par l'individu qui la produit, dans le référentiel spécifique des idées pédagogiques, écrire l'histoire reviendrait de fait à en rechercher les auteurs: toute demande ou question concernant les idées pédagogiques porte, tout d'abord, à retrouver leur auteur. Un des principaux effets induit par cette quête de reconnaissance en paternité (maternité ?) accomplie par les historiens, servirait dans une telle perspective à déterminer des «classiques» auxquels on se doit de faire référence, dans un environnement culturel donné. Mais qui décidera que tel auteur mérite d'entrer dans la mémoire collective des éducateurs, et selon quels critères?

En opposition à cette vision personnaliste de l'histoire des idées, théoriciens et philosophes montrent de concert que l'on peut parler de pédagogie en dehors de tout rapport existentiel. Le monde des théories se satisfait peu du caractère poétique et de «l’incarnation pédagogique».

Une autre conception de l'histoire conteste l'acception selon laquelle la genèse et le développement des idées pédagogiques suivraient un parcours linéaire des acteurs, les pédagogues, vers les facteurs, la société. Ces mêmes idées transfusent incontestablement, bien que de façon différente, de la culture (conçue comme conjonction de facteurs) vers les acteurs. Les pédagogues sont en effet des êtres curieux de leur temps, qui prennent en compte dans leur quête de mieux-être, outre les apports des différentes sciences humaines, des outils, des savoirs, des principes méthodologiques issus de champs théoriques fort disparates, utiles à leur réflexion et à leur action éducatives. À cet égard, toute idée pédagogique, dans sa valeur de synthèse provisoire, déborde le cadre même de l'éducation. Ce constat rend plus ardue encore la tâche de l'historien qui voudrait s'engager dans une description précise de liens de cause à effet. Évaluer l'influence de tel ou tel référentiel sur la modification des conceptions éducatives de l'acteur, ou sur les reformulations successives de ce qui fait son originalité en tant que pédagogue, revient au premier regard à poser une problématique insoluble. Pour toute période historique se pose, pour peu que l'on accepte une perspective critique, la question de l'importance et de la profondeur du lien existant entre les idées pédagogiques et leur influence sur la construction ou la réélaboration d'une réalité éducative donnée, ainsi que son inévitable corollaire: la détermination de ce qui est de première importance par rapport à ce qui reste superficiel ou éphémère, ainsi que les critères qui fondent une telle classification des facteurs. Vérifier que, de manière incontestable, une idée pédagogique ou la combinaison de plusieurs d'entre elles aient eu des retombées sur les pratiques éducatives quotidiennes, qu'elles soient institutionnelles ou domestiques, conduit le chercheur à une posture alternative à celle du biographe, en se plaçant résolument du côté des facteurs.

\section{Repères}

«Choisir, c'est renoncer» nous disent les psychanalystes. Ainsi l'historien des idées pédagogiques se voit contraint d'affirmer ses choix épistémologiques en défendant 
explicitement les options retenues, et les enjeux qu'ils représentent dans la manière de «dire» l'histoire. Dans cette perspective, la pensée complexe définie par Edgar Morin ainsi que l'herméneutique vue par Paul Ricœur constituent autant de référentiels de tout premier ordre. L'application d'outils méthodologiques issus de ces champs à l'histoire des idées pédagogiques nous conduira à nous démarquer de positions déterministes, au profit de déterminations multiples, abandonnant l'idée de causalité linéaire impliquant que, derrière une complexité apparente, se dissimule une logique régulatrice des phénomènes humains. Nous tenterons également de définir en quoi l'approche anthropologique, "actualisation d'innombrables virtualités humaines », permet de rendre toute leur place aux sujets, aux côtés des paramètres économiques et sociaux traditionnellement valorisés par les historiens positivistes:

l'histoire n'est pas seulement une succession d'évènements, de bruit, de fureur ou de processus économiques mécaniques. L'histoire devient manifestation de diverses potentialités humaines. C'est une façon de nous mettre en relation avec l'anthropos, la condition humaine ${ }^{3}$.

\section{Éloge de la complexité}

Quel chercheur en histoire des idées n'a pas ressenti une forme de découragement devant l'inextricable fouillis, le désordre apparent auquel conduit le repérage des sources à l'orée d'une recherche nouvelle? La science a besoin d'ordre: d'où la tentation première d'occulter le désordre, de hiérarchiser les corpus documentaires, en écartant tout ce qui induirait ambiguïté et incertitude. Cette opération historiquement nécessaire, qui consiste à produire une analyse claire et pertinente du passé, s'appuyant si possible sur l'administration de la preuve (documentaire), conduit bien souvent à la production d'interprétations aveugles et à une forme de cécité plus ou moins bien assumée par celui qui les pose.

L'une des modalités qui permettrait de réduire la complexité pour remettre de l'ordre dans l'histoire serait sans doute de s'en remettre aux experts pour traiter la même réalité à des niveaux référentiels différents: il y aurait ainsi une analyse économique, psychologique, sociale, etc. d'un même référent. L'histoire s'écrirait dans le cadre de cette multiréférentialité : la combinaison entre elles d'analyses unidimensionnelles, parcellaires et spécialisées, permettrait de produire des synthèses encyclopédiques, réputées exhaustives. Morin récuse une telle confusion entre complexité et complétude:

La totalité, c’est la non-vérité. Nous sommes condamnés à la pensée incertaine, à une pensée criblée de trous, à une pensée qui n'a aucun fondement absolu de certitude. Mais nous sommes capables de penser dans ces conditions dramatiques ${ }^{4}$.

3. Morin 1999, 352-353.

4. Morin 1990, 93. 
Tout chercheur qui se fait «monographe épistémique» aspire à la complétude. La différence d'avec la multidimensionnalité propre à la juxtaposition d'expertises, c'est que le monographe épistémique assume seul la responsabilité des regards croisés qu'il porte sur son référent. Aspirer à la complétude tout en acceptant la complexité, c'est assumer en conscience l'incertitude, l'idée utopique d'un savoir global, tout en conservant à l'eu-topos ses deux acceptions originelles: une île inaccessible certes, mais aussi un espace où l'on est bien... C'est aussi résister à la perspective de rationalisations arbitraires qui enferment les faits historiques dans un système supposé cohérent. Une forme (classique au demeurant) de rationalisation délirante consiste à ne retenir que les indicateurs qui confortent un système explicatif, en écartant tout ce qui pourrait le contredire. Se référer à la complexité place ainsi le chercheur dans une tension inconfortable: entre nécessaires simplifications, hiérarchisations, sélections et aspirations à la complétude; entre la volonté de dire une histoire achevée et l'imperfection, acceptant l'incertitude propre à toute tentative de rationalisation des phénomènes humains.

Le principal défi, inhérent à ces allers et retours entre simplicité et complexité, est bien de "s'y retrouver », de ne pas perdre le fil, tissant comme Pénélope une toile cohérente mais toujours inachevée, sans cesse remise sur le métier au cours du temps, complexe à défaut d'être complète. Le risque serait de «s'y perdre», dans une confusion des sentiments où l'on ne sait plus ce que l'on veut ni où l'on va.

\section{Double herméneutique : traduire et interpréter}

«S’y retrouver» nécessite en particulier l'utilisation de cartes et de boussoles, propres à baliser un itinéraire incertain. François Dosse ${ }^{5}$ propose à cet effet, en référence aux travaux de Ricœur, une «double herméneutique», conçue comme un processus d'alternance entre traduction et interprétation. Cette méthodologie, féconde pour l'historien, le situe au cœur d'un débat dialectique et mutuel entre deux pôles: l'explicatif (traduction de traces sémiotiques différenciées) et le compréhensif (mise en relation des résultats de l'analyse en vue de construire un réseau de signification cohérent). Cette tension permanente entre explication et compréhension, la nécessité d'un va-et-vient continuel, permet de nourrir la recherche du potentiel propre à ces deux pôles. La visée explicative induit une attitude critique, distante. Le mouvement de compréhension implique l'immersion, l'appartenance, l'ouverture à de nouveaux possibles: "Interpréter, c'est imaginer un ou des mondes possibles déployés par le texte, et c'est agir...» ${ }^{6}$.

Le concept ne s'oppose plus au vécu pour le disqualifier, et la quête de sens se réalise à partir de «médiatrices imparfaites» dans une «dialectique inachevée » toujours

\footnotetext{
5. Dosse 1999, 317.
}

6. Dosse 1999, 321. 
ouverte à un sens nouveau. Cette ouverture sur la temporalité, sur la chaîne des générations inscrite dans la trame de l'historicité, s'oppose à la coupure épistémologique devenue un absolu du paradigme animé par une prétention scientiste ${ }^{7}$.

\section{Le retour du sujet}

La tension herméneutique précédemment décrite permet-elle de déterminer des modèles explicatifs globaux, véritables règles de grammaire organisatrices du sens de l'histoire? Certes non, si l'on se réfère au paradigme structuraliste, dominant dans les années soixante-soixante-dix, dont l'ambition fut de saisir la réalité de manière objective et scientifique. La revendication théorique, qui caractérise les auteurs qui s'y réfèrent, sous-tend la conception fondamentale d'une vérité scientifique présente mais cachée, qu'il s'agit de démasquer. Éthique du soupçon et nécessité du décentrement, propres à cette approche, renvoient les acteurs hors du champ de l'expertise, exilés des terres d'investigation comme des indicateurs instables et aléatoires, principales sources d'illusions et de mirages. Si l'objet de la connaissance historique est de définir l'immuable, les invariants dans la détermination des conduites humaines, le sujet redevient objet, enfermé, contraint par le déterminisme des lois et le jeu des facteurs. Certes, la détermination de ces mécanismes reste un des objets privilégiés de l'historien, mais un objet parmi d'autres. Car l'histoire n'est pas écrite à l'avance, et c'est justement dans la capacité des acteurs à s'extraire des conditionnements de toute nature que se construit l'essentiel de l'historicité, qui échappe aux structuralistes. Éternelle dualité du singulier et du général. Accepter d'être «travaillé» par la tension herméneutique implique, à l'inverse, une mise à l'écoute du récit des acteurs, en leur reconnaissant une compétence singulière à analyser leur propre situation. Le «sens commun» exclu par les structuralistes est réintégré dans le champ de la recherche par l'herméneute. L'historien se fait alors ethnologue, en s'attachant à suivre au plus près les interprétations internes fournies comme autant de matériaux par les acteurs. Ce travail interprétatif interne constitue ainsi un gisement potentiel de savoirs, qui ne doit pas d'emblée être réfuté par un travail interprétatif externe qui conduirait à sa disqualification.

L'interprétation externe doit se bâtir sur le terreau de l'explication, ou, pour mieux dire, d'explications multiples et croisées. Expliquer plus pour comprendre mieux, telle pourrait être la devise de l'herméneute. Elle engage à une forme de compagnonnage avec l'acteur et son histoire, comme elle exige un examen précis des marqueurs internes et externes qui situent son niveau d'implication dans le débat d'idées. L'historicité ne se substitue pas à la structure; les savoirs issus de l'une viennent féconder ceux de l'autre. Le retour au premier plan d'actes explicités et réfléchis place celui qui les pose au centre d'une quête interprétative qui ne se satisfait pas de l'histoire

7. Dosse 1999, 320. 
convenue. Ce retour de l'acteur ne remet nullement en cause le primat du regard critique, de la mise à distance et de l'objectivation. Il ne s'agit pas non plus d'une quête de l'exotisme, mais plutôt d'une démarche «qui vise à l'appropriation des diverses sédimentations de sens léguées par les générations précédentes, des possibles non avérés qui jonchent le passé des vaincus et des muets de l'histoire ${ }^{8}$.

\section{Interprétations conflictuelles}

Si la multiplicité, le désordre apparent, l'embrouillamini des sources constituent pour le chercheur en histoire des idées une cause probable de découragement initial, il en est une autre, tout aussi pernicieuse, que questionne Daniel Hameline dans un ouvrage récent ${ }^{9}$ autour de la métaphore du bègue: «Tout est dit et l'on vient trop tard, depuis sept mille ans qu'il y a des hommes et qui pensent». Rien de nouveau sous le soleil... L'aphorisme de La Bruyère nous condamne à réécrire l'identique à l'infini, en ressassant les mêmes litanies, les mêmes refrains:

Tout est dit. Voilà effectivement une bien puissante image de l'origine. Mais n'estce pas énoncer plutôt qu'il n'est pas d'origine puisque les jeux sont faits, que rien ne peut désormais commencer qui ne soit barré d'avance? Tout possible est définitivement altéré. «L'ancien» est réputé le lieu du «meilleur», un «ancien» qui est trop notre idée pour s'avérer mythique, ce qui le sauverait de notre entreprise complaisante ${ }^{10}$.

Quiconque veut apporter une contribution originale sur l'autel de l'histoire des acteurs est-il condamné à faire revivre des figures oubliées, pédagogues apparemment de second ordre, en les faisant passer pour ce qu'ils ne sont pas? L'entrée par les facteurs confronte l'infortuné chercheur aux savoirs académiques et à la doxa. Qui peut prétendre réécrire aujourd'hui l'histoire des institutions de formation des maîtres en France? L'école maternelle de la République peut-elle sérieusement avoir eu des racines piétistes moraves? La liste des exemples est innombrable et à première vue décourageante. Pourtant l'impuissance du bègue fait aussi paradoxalement sa force, qui réside dans sa résistance et son entêtement:

[...] le bégaiement nous signifie qu'il y a, bien évidemment, quelque chose à dire. Et quelque chose d'important. Car bégayer, c'est insister. C'est s'instaurer impuissant dans l'insistance et donner par là toute sa mesure au malentendu ${ }^{11}$.

\footnotetext{
8. Dosse 1999, 325.

9. Hameline 2002, 3-7.

10. Hameline 2002, 5 .

11. Hameline 2002, 3.
} 
La démarche herméneutique vient alors au secours du bègue à travers le conflit des interprétations théorisé par Ricœur. Ce conflit interprétatif est une porte ouverte sur une construction plurielle, mouvante et dynamique des savoirs, une conception en tension de la vérité.

L'herméneute bredouille parce qu'il est médiateur entre deux mondes déployant son art dans deux directions. Il cherche à retrouver le fil d'une communication rendue inaudible par le gouffre spatio-temporel, devenue obscure par l'évolution des contextes et des langages. La distance, l'écart avec le présent sont tout autant des obstacles que de puissants stimulants pour celui qui cherche. Le recueil, la traduction et l'authentification des sources permettent de reconstruire jusque dans les détails ce qui entre dans le décodeur de la mémoire sous forme d'input; la combinaison de cet explicatif avec l'interprétation externe, le compréhensif, restaure «en clair», non à l'identique mais de manière originale, un réseau de significations brouillé jusqu'alors. Ainsi, si le travail d'analyse, pour peu qu'il suive une méthodologie rigoureuse, relève de l'administration de la preuve, c'est bien l'output, la reconstruction du sens dans ce qu'elle a de singulier qui fait problème. Le conflit des interprétations suppose une conception de la vérité en tension.

Après avoir jeté des ponts par-dessus le fleuve du temps pour entrer en relation avec l'autre, semblable différent, il faut en jeter de nouveaux avec ses contemporains, pour échanges. Ces échanges réciproques et contradictoires à la recherche du sens caché, dans une perspective de démythologisation, participent d'une construction de sens plurielle.

Entre chronique, récit d'une approche éducative singulière inscrite dans des limites spatio-temporelles déterminées et analyse du contenu de ce qu'un auteur a rêvé être l'éducation sur les terres de l'utopie, entre simplification extrême et complexité parfaite, se dégage un espace d'étude étroit, aux limites incertaines, entre discours sur les idées pédagogiques et histoire des idées pédagogiques. Investir l'espace «où s'invente l'humanité dans le champ des possibles» résonne comme une invitation à «revisiter, à partir du passé, les multiples possibles du présent, afin de penser le monde de demain ${ }^{12}$. Nous suivons A. Novoa lorsqu'il affirme que:

La responsabilité de l'historien ne s'acquitte pas dans les réponses, mais surtout dans les questions. La valorisation d'une histoire des problèmes permet de situer son compromis intellectuel dans le temps et l'espace d'aujourd'hui ${ }^{13}$.

Une des finalités essentielle des résultats de la recherche historique nous semble ainsi être «d'interroger» l'apparente cohérence de la réalité contemporaine, pour aider à distinguer, dans un tissage complexe de facteurs hétérogènes, les "possibles » de chacun d'entre eux, et cela au-delà des systèmes explicatifs compartimentés des différentes sciences sociales. Elle induit en particulier l'opportunité de repenser le rapport de l'histoire avec les autres sciences.

12. Dosse 1999, 326.

13. Novoa 1998, 21. 


\section{Conclusion}

Aucune rationalité scientifique ne pouvant prétendre démêler, à elle seule, telle ou telle tentative de synthèse empirique du rapport théorie pratique, les champs pédagogiques, mais encore philosophiques, théologiques, etc., dès lors qu' ils jouent une partition en mode mineur, sont aussi nécessaires que l'histoire qui joue, certes en mode majeur, à l'élaboration et aux enrichissements successifs du réseau hypertextuel tissé par les historiens des idées pédagogiques.

Trois dimensions, jugées complémentaires, devront dès lors être prises en compte:

- une dimension théorique d'abord: la nécessaire convergence entre différents référentiels ouvre la voie à une histoire des idées pédagogiques ne devant aux autres domaines scientifiques que des emprunts de bon voisinage qui devraient constituer la vie ordinaire des théories et des disciplines;

- une dimension pratique: l'appréhension d'un objet de recherche dans un cadre pluridisciplinaire induit la maitrise de concepts et de principes méthodologiques particuliers à chacune d'entre elles. L'appropriation de ceux-ci constitue un prérequis déterminé par l'utilité qu'ils représentent au service de la recherche en cours;

- une dimension philosophique: une telle posture épistémologique construite autour de l'idée d'unité, par opposition à celle d'expertise, remet peut-être à l'honneur une approche romantique et progressiste de l'histoire, contre les conceptions philosophiques des thèses de Comte sur les trois états de la science: théologique, métaphysique et positif.

Disons, pour conclure, que l'enjeu majeur d'un développement du référentiel «histoire des idées pédagogiques» au sein du monde de l'éducation nous semble être d'éviter le passage, chez les éducateurs en général, de l'héritage à la rémanence. L'héritage inscrit l'humain dans une filiation, et donc dans une culture. La rémanence aveugle et permet toutes les formes de manipulations. L'héritage permet l'exercice d'une liberté individuelle et intellectuelle: ainsi est-il possible de refuser tout ou partie d'un héritage.

Refuser de prendre en compte des conceptions pédagogiques anciennes, des pratiques du passé (dépassées?), conduit à une forme de myopie dans la compréhension du présent. La finesse dans l'analyse des réalités éducatives contemporaines reste très dépendante de clés de lecture historiques: la perte de ces clés nous tient isolés d'un réseau de significations, solitaires en quête de solidarités.

Nous suivons Daniel Hameline lorsqu'il affirme tout l'intérêt de ces solidarités historiques pour le chercheur, dans une quête de l'identique qui paradoxalement révèle, au sens photographique du terme, des éléments de dissemblance et qui, loin de s'imposer comme des modèles, nous permettent d'exister comme uniques, en tant que semblables / différents: 
L'histoire nous instruit sur nous-mêmes parce qu'elle nous restaure à l'identique, avec, quelque part, le frémissement d'un juste assez de dissemblance pour qu'il y ait doute sur le passé et, dès lors, doute sur le présent: un interstice s'ouvre, et comme une échappée belle ${ }^{14}$.

L'histoire n'est pas écrite à l'avance, dans le cadre d'un déterminisme des facteurs où les acteurs ne seraient que de simples figurants. Les politiques éducatives peuvent être plurielles, mais il est du ressort des acteurs de décider in fine si l'acte d'éduquer concerne encore l'affirmation de l'humanité dans l'homme... À l'heure des choix, les pédagogues, qui génèrent, structurent mais aussi critiquent les idées éducatives, peuvent contribuer à ne pas perdre de vue les principes fondateurs d'une éducation humaniste au centre de laquelle l'homme se tient debout, comme un tout insécable.

Loïc Chalmel

CIVIIC

Université de Rouen

\section{Références bibliographiques}

Dosse F. (1999), «La méthode historique et les traces mémorielles», in Relier les connaissances. Le défi du XXIe siècle, E. Morin (dir.), Paris, Seuil.

Hameline D. (2001), "Connaissez-vous Stuber? Variation sur: avez-vous lu Baruch?», in Jean-Georges Stuber (1722-1797). Pédagogie pastorale, L. Chalmel, Berne - Paris, Peter Lang.

Hameline D. (2002), L'éducation dans le miroir du temps, Lausanne, LEP Loisirs et pédagogie.

Marrou H.-I. (1948), Histoire de l'éducation dans l'Antiquité, Paris, Seuil.

Morin E. (1990), Introduction à la pensée complexe, Paris, ESF.

Morin E. (1999), Relier les connaissances. Le défi du XXI' siècle, Paris, Seuil.

Novoa A. (1998), Histoire \& comparaison (essais sur l'éducation), Lisbonne, Educa.

Ricceur P. (1969), Le conflit des interprétations. Essais d'herméneutique, Paris, Seuil.

Ricceur P. (1986), Du texte à l'action. Essais d'herméneutique II, Paris, Seuil.

RodAri G. (1979), Grammaire de l'imagination, Paris, Messidor.

14. Hameline 2002, 6 . 



\section{LES RECHERCHES EN SCIENCES DE L'ÉDUCATION ET DANS LES DISCIPLINES ÉPONYMES}

- La psychologie - 



\section{DU CÔTÉ DES RECHERCHES CLINIQUES D'ORIENTATION PSYCHANALYTIQUE EN SCIENCES DE L'ÉDUCATION}

Résumé: J'interpréterai pour ma part la question posée en me référant à une seule branche de la psychologie: la psychologie clinique; je proposerai ainsi quelques pistes de réflexion concernant la mise en perspective du courant de recherches cliniques d'orientation psychanalytique que je soutiens dans le champ des sciences de l'éducation avec le courant de recherches cliniques soutenu par des chercheurs relevant de la discipline psychologie. J'indiquerai que, dans notre champ, nous nous inscrivons dans un courant Pédagogie et psychanalyse dont les premiers contours ont été dessinés par Freud et que cette appartenance nous allège peut-être de certaines contraintes en même temps qu'elle définit notre lien singulier à la psychanalyse. Je montrerai que cela se traduit concrètement au plan de la méthodologie de recherche en induisant notamment des différences sur la question des hypothèses, sur celle des élaborations contre-transférentielles du chercheur ainsi que sur les questions de validation; je ferai ainsi l'hypothèse que cela nous donne peut-être un peu plus de liberté dans notre posture de chercheur. Je m'appuierai, pour ce faire, sur la note de synthèse que nous avons publiée dans le numéro 151 de la Revue française de pédagogie en 2005 et sur les nombreuses communications présentées au colloque Cliopsy que nous avons organisé à la Sorbonne en novembre 2006 .

Mots clés: psychologie clinique, sciences de l'éducation, psychanalyse, discipline, rapport au savoir, transfert didactique.

La question qui nous a été adressée pour les exposés de ce jour, nous l'avons vu, déjà, avec les interventions précédentes, a été traduite ou interprétée par les chercheurs sollicités pour y répondre de différentes manières. Pour ce qui me concerne, la question était: "Psychologie: fait-on de la recherche de la même façon dans la discipline éponyme et en sciences de l'éducation?». J'aurais pu intituler mon intervention: «Du côté des recherches cliniques d'orientation psychanalytique en sciences de l'éducation ou comment transformer un état de fait qui en apparence nous est défavorable en ressource?». En lien avec mon champ de compétences, j'ai choisi de restreindre la portée de la question qui nous était posée en ne m'intéressant véritablement qu'à la branche de la psychologie la plus proche de ma démarche de recherche, à savoir la psychologie clinique. Ce point de départ étant posé, je tenterai dans un 
premier temps de dire de quoi est constitué l' «observatoire» d'où je tiens les propos que j'avance, le lieu à partir duquel je propose des éléments pouvant apporter un certain éclairage pour répondre à la question. En troisième lieu, je mettrai l'accent sur les dimensions institutionnelles de la question, en me souvenant des points sur lesquels Jacky Beillerot ${ }^{1}$ aurait été vigilant s'il avait pu être parmi nous. Dans une deuxième partie de l'exposé, je me placerai sur un plan plus épistémologique et je m'attacherai à dégager certaines spécificités de nos recherches cliniques en sciences de l'éducation, en montrant que la pratique clinique qui soutient nos recherches ne se penche pas sur la même facette des sujets auprès desquels nous intervenons que les psychologues cliniciens; nous nous attachons à travailler au plan psychique non sur la personnalité des professionnels auprès desquels nous menons nos recherches ou que nous accompagnons mais avant tout avec la part professionnelle de ces personnes. Cette spécificité entraîne la construction d'objets nouveaux, de nouvelles méthodes d'investigation ou tout du moins des transformations des méthodes classiques ainsi que l'émergence de nouvelles notions en voie de conceptualisation. Pour terminer, je proposerai une hypothèse pour indiquer que les conséquences de la problématique institutionnelle liées à notre discipline "sciences de l'éducation ", alors qu'elles pouvaient nous apparaître comme défavorables à première vue et qu'elles ont pu s'avérer plutôt disqualifiantes pour nous dans un premier temps, peuvent être considérées à l'inverse comme des atouts pour construire nos recherches dans un plus grand espace de liberté à partir de l'autonomie de notre discipline par rapport à la discipline éponyme. Voilà, en quelques mots résumé le message que je voudrais transmettre. Dans ce court texte, je vais reprendre ces quelques points sans pour autant les développer autant qu'il serait souhaitable, tout en indiquant au passage les références bibliographiques sur lesquelles je m'appuie pour cette argumentation.

\section{Mon «observatoire»}

Je suis professeure en sciences de l'éducation depuis 1991 à Paris X Nanterre dans une Unité de formation et de recherche (UFR) de sciences psychologiques et sciences de l'éducation. Pour autant, les liens noués avec mes collègues professeurs de psychologie clinique, psychanalystes ou professeurs de psychiatrie se sont produits le plus souvent hors de mon UFR d'appartenance, à l'occasion de colloques et de congrès extérieurs où je suis invitée, ou dans des jurys de thèse ou de masters recherche soutenus en psychologie et concernant des objets de l'éducation pour lesquels j'ai été sollicitée depuis quelques années, après la sortie de mon ouvrage aux PUF en 2001 Les enseignants entre plaisir et souffrance. Un autre espace-temps de cet observatoire est celui que nous avons nous-mêmes construit en organisant deux rencontres internationales, l'une en novembre 2003 à Paris X, l'autre en novembre 2006 à Paris V, en

1. Que dire sur lui? Qu'il a énormément œuvré pour la constitution / légitimation du courant clinique dans les sciences de l'éducation? 
nous appuyant sur les ressources (à l'initiative?) des deux laboratoires de sciences de l'éducation de ces universités, le CREF (Centre de recherche éducation et formation à Paris X) et l'équipe EDA (Éducation, didactique et apprentissage à Paris V). En 2003, la manifestation a rassemblé quatre-vingts participants sous la forme de plusieurs tables rondes; en 2006, deux cents participants étaient présents et une soixantaine de communications ont été proposées, communications émanant de chercheurs en psychologie et de chercheurs en sciences de l'éducation se recommandant tous d'une forme de clinique d'orientation psychanalytique. Pour ma part, à ce jour, j'ai fait soutenir une cinquantaine de mémoires de recherches et j'ai eu l'occasion d'accompagner une vingtaine de thèses cliniques.

\section{La part de ma subjectivité}

Peut-on parler de subjectivité dans ma manière d'analyser ces phénomènes? Sans doute, dans la part de méconnaissance que peut révéler mon propos et aussi au niveau des interprétations que j'en propose. Néanmoins, mon observation repose sur certains éléments tout à fait objectifs: je ne suis pas "psychologue», au sens où je n'ai pas été formée par un cursus en psychologie mais que j'ai suivi un cursus de sciences de l'éducation (comme deuxième formation après une formation initiale en mathématiques et plusieurs années d'exercice au niveau de l'enseignement supérieur de cette discipline). On peut facilement imaginer que cette trajectoire singulière aboutit à me donner un point de vue différent de celui d'un autre professeur en sciences de l'éducation soutenant la même approche clinique que moi qui, lui, est «psychologue », je veux parler de mon collègue de Paris V, Bernard Pechberty. Le travail que je présente ici n'existerait pas sans les échanges fructueux entre nous qui durent maintenant depuis plusieurs années et qui m'ont sensibilisée à cette différence de points de vue. D’emblée, je dirais que ce collègue est, par exemple, mieux accueilli que moi par les psychologues universitaires, étant davantage « des leurs», relié à leur communauté d'appartenance. En revanche, j'ai été témoin privilégié du chemin qu'il a dû faire pour entrer dans notre culture «sciences de l'éducation» et y trouver sa voie. C'est bien la mise en perspective de nos trajectoires respectives qui aujourd'hui tendent à se rejoindre au sein des sciences de l'éducation qui m'a aidée à mettre en lumière certaines proximités et différences.

\section{Appartenances disciplinaires}

J'en arrive ainsi au point suivant de mon argument, la question institutionnelle, celle de l'appartenance disciplinaire. Notre discipline «sciences de l'éducation » a aujourd'hui quarante ans d'existence, et c'est à ce propos que nous sommes réunis, mais estimons-nous qu'elle a acquis une reconnaissance au plan de la consistance de son épistémologie ou de ses épistémologies? Ce que l'on peut constater, en tout cas, c'est qu'entre les sections du CNU, il ne lui est pas reconnu une égale dignité de fait avec les autres disciplines, comme l'énonce Y. Dutercq dans sa propre contribution à cette rencontre. Nous avons tous remarqué qu'il est possible d'être «qualifié» en 
$70^{\mathrm{e}}$ section du CNU avec une thèse en psychologie alors que l'inverse est quasiment inenvisageable. Sans doute faut-il convenir que les conditions pour obtenir le titre de psychologue et la protection de ce titre ne rendent pas les choses faciles en termes de mutualisations entre nos cursus. Je l'ai particulièrement éprouvé au niveau des tentatives de mutualisations entre nos masters professionnels. Un autre indice de cette hiérarchie entre les deux disciplines m'est fourni en observant la manière dont nos collègues se désignent eux-mêmes dans les quatrièmes de couverture de leurs ouvrages, ou au moment de certaines émissions de télévision ou de radio dans lesquelles ils sont invités ou encore lors des entretiens dans la presse. Se disent-ils, se disent-elles professeurs en sciences de l'éducation ou le plus souvent psychologues, ou sociologues? Regardons d'ailleurs leur présentation comme auteurs pour le Dictionnaire encyclopédique de l'éducation et de la formation (2005 pour la troisième édition). Et notons de plus à qui l'on a fait appel pour rédiger les notices relevant de la psychologie, par exemple, dans ce dictionnaire; on peut aussi consulter dans le Traité de sciences et pratiques de l'éducation dirigé par J. Beillerot et N. Mosconi la rubrique dans laquelle se présentent les auteurs qui ont été sollicités.

Or, si nous-mêmes, nous ne portons pas plus fièrement notre appartenance disciplinaire, qui le fera à notre place? Rendons-nous compte que nous donnons ainsi prise aux tenants d'autres disciplines que cela pourrait, dans certains cas, arranger de sous-estimer nos spécificités, dans le jeu des concurrences disciplinaires. J'en viens à des considérations plus liées aux questions de "scientificité», lesquelles nous entraînent vers des réflexions épistémologiques.

\section{Réflexions épistémologiques}

Pour cette deuxième partie, je me référerai principalement à ce que nous avons écrit dans la note de synthèse parue en 2005 dans la Revue française de pédagogie, intitulée «Recherches cliniques d'orientation psychanalytique dans le champ de l'éducation et de la formation ". J'inviterai le lecteur à se reporter à ce texte s'il souhaite entrer dans les détails de l'argumentation, car c'est un texte écrit dans lequel nous avons pris position sur plusieurs points nodaux concernant notamment la légitimité des recherches cliniques d'orientation psychanalytique menées en sciences de l'éducation et leur spécificité. Premièrement, nous estimons que les chercheurs de notre champ, souvent praticiens ou ex-praticiens de l'éducation eux-mêmes, sont légitimes à faire usage de la psychanalyse dans leurs recherches en tant que corpus théorique lié à une praxis, dès lors qu'ils / elles s'engagent à acquérir une certaine expérience du travail psychique et sans pour autant être labellisés comme psychologues cliniciens et / ou comme psychanalystes.

Deuxièmement, lorsque nous, chercheurs cliniciens en sciences de l'éducation, sommes engagés dans une pratique clinique, c'est le plus souvent du côté du registre professionnel des personnes que nous nous situons, du côté des difficultés liées à l'école pour les élèves chez les enfants, et du côté de la souffrance au travail chez les 
professionnels de l'éducation et de la formation auprès desquels nous menons nos recherches et / ou que nous accompagnons. Bien entendu, notre approche ne serait pas clinique si elle nous conduisait à opérer un clivage entre le professionnel et le personnel, mais il s'agit plutôt d'une question de centration de notre regard et de notre écoute. L'entrée de nos travaux est toujours la question de l'identité professionnelle et l'analyse de la part professionnelle du sujet. Pour ma part, lorsque j'en viens, par exemple, à parler de «soin psychique» aux enseignants (intitulé de mon dernier article pour la revue Cliniques méditerranéennes ${ }^{2}$ ) il s'agit bien de «soins » à la part professionnelle des acteurs éducatifs et non de proposer une thérapie personnelle à ces professionnels. Ainsi, j'estime que nous sommes en train de développer des dispositifs spécifiques pour ce que je nomme une clinique professionnelle du lien.

\section{Dimensions institutionnelles}

Troisièmement, à mes yeux, cette spécificité nous a entraînés vers des différences dans l'évolution de nos méthodologies de recherche. Nous avons par exemple beaucoup développé l'usage de l'entretien clinique de recherche ${ }^{3}$, à partir de la méthodologie proposée par M.-F. Castarède ${ }^{4}$ dans le livre dirigé par Colette Chiland. Sans doute, notre manière d'utiliser cette forme d'entretien de manière privilégiée, plutôt que d'utiliser par exemple des tests projectifs, nous est singulière. De la même façon, nous tentons aujourd'hui de transposer des méthodes d'observation psychanalytique dans notre champ, en particulier dans le cadre de l'observation des pratiques enseignantes, sans ignorer les débats qu'elles suscitent au sein des courants psychanalytiques, mais aussi en tenant compte des avancées spécifiques de la question de l'observation dans notre champ ${ }^{5}$.

$\mathrm{Au}$ niveau de la manière de poser les problématiques de recherche, a contrario de ce que l'on peut par exemple lire dans le texte d'Odile Bourguignon sur le processus de recherche ${ }^{6}$, nous ne formulons pas d'hypothèses de recherche a priori, sinon, bien sûr, des hypothèses théoriques concernant les postulats ou les fondamentaux de la psychanalyse (inconscient freudien, transfert, etc.). Nous prenons acte, en quelque sorte, de la dimension heuristique de la démarche et de ses limites, à savoir qu'elle n'a pas la capacité de pouvoir vérifier ou infirmer des hypothèses de recherche, comme cela est recommandé dans certains textes à l'intention des étudiants en psychologie ${ }^{7}$. De même, à propos des questions de validation, nous développons des réflexions à propos des caractéristiques de l'écriture des résultats des recherches cliniques, à la

2. Blanchard-Laville 2007.

3. Yelnik 2005.

4. Castarède 1989.

5. Chaussecourte 2003 et 2006.

6. Bourguignon \& Bydlowski 1995.

7. Ghiglione \& Richard, 1993. 
suite de Mireille Cifali ${ }^{8}$ et de Claude Revault d'Allonnes ${ }^{9}$, pour tenter de formaliser la manière spécifique de communiquer nos résultats en ce domaine; il s'agit en effet de ne pas renoncer à une manière argumentative, tout en respectant le fait qu'il nous est impossible d'aller vers ce qu'ordinairement on appelle l'administration de la preuve et le type de généralisation proposés classiquement. Nous nous situons ainsi plutôt du côté d'une éthique de la conviction, en évitant d'un autre côté ce qui serait de l'ordre de la violence de l'interprétation ou de la «violence du sens imposé» comme l'écrivait Claude Revault d'Allonnes ${ }^{10}$.

Si enfin nous allons sur le plan de l'émergence de nouvelles notions, nous en reconnaissons au moins une, portée et développée en partie par notre équipe à Paris X et mise en lumière par Jacky Beillerot, celle de «rapport au savoir " ${ }^{11}$; notion dont j'ai pu apercevoir qu'elle est encore relativement ignorée au sein de la discipline psychologie ou tout du moins peu travaillée alors qu'elle aurait été utile à certains chercheurs, auteurs de mémoires et de thèses en psychologie, que j'ai eus à évaluer et dont j'ai estimé qu'ils auraient tiré grand bénéfice de la connaissance de nos travaux dans ce domaine.

Sur un plan personnel, je me trouve engagée, au-delà de la transposition de certains concepts psychanalytiques dans mon champ de recherches, dans une construction théorique originale, notamment pour ce qui concerne la mise en intelligibilité des phénomènes psychiques liés aux pratiques enseignantes dans l'espace d'enseignement. Je peux commencer à y avancer une notion comme celle de «transfert didactique», qui témoigne de la spécificité des phénomènes transférentiels et contretransférentiels lors de l'acte d'enseigner, en lien avec le rapport au savoir de celui ou celle qui enseigne ${ }^{12}$ et celle de "holding didactique», pour désigner la manière d'habiter au plan psychique le geste de «tenir» un groupe-classe ${ }^{13}$. J'ai pu montrer dans mes travaux, à plusieurs reprises, la genèse de cette conceptualisation en indiquant les éléments sur lesquels elle s'appuie et desquels elle a dû s'émanciper par la suite, en s'autorisant à penser des retournements de perspective ${ }^{14}$.

Ainsi je dirais pour résumer que ce sont à la fois nos objets, nos méthodes et les concepts qui se transforment chemin faisant dans notre différenciation disciplinaire. Je terminerai par la question qui, sans doute, nous distingue le plus en ce moment de nos collègues psychologues cliniciens, c'est celle qui se rapporte aux élaborations contre-transférentielles du chercheur vis-à-vis de ses questions de recherche. Au-delà de son implication ${ }^{15}$, nous sommes amenés, pour évoquer la prise en compte de la subjectivité du chercheur à l'égard de sa recherche, à parler, à la suite

\footnotetext{
8. Cifali 1999.

9. Revault d'Allonnes 1989.

10. Revault d'Allonnes 1998.

11. Beillerot 1987, 1996, 2000.

12. Blanchard-Laville 2005.

13. Blanchard-Laville 2001.

14. Blanchard-Laville 2003.

15. Ardoino 2000.
} 
de G. Devereux ${ }^{16}$, des mouvements contre-transférentiels du chercheur, mouvements qui, pour nous, sont à la fois le moteur et l'obstacle dans notre manière de faire de la recherche. Nous développons actuellement nos réflexions à ce sujet et notamment, nous réfléchissons à la manière de soutenir la tension forte que cela implique pour les chercheurs qui s'engagent sur cette voie; notamment à partir de l'examen des modalités par lesquelles il est possible de les accompagner, à la fois au niveau des élaborations nécessaires dans leur avancée et dans l'écriture de certaines de ces élaborations en lien avec la production de connaissances. C'est ainsi que, adoptant les messages de Georges Devereux à la lettre, je préconise que ces élaborations doivent figurer d'une certaine manière dans ce qui est rendu public de nos résultats de recherche dans le but, puisque nous ne pouvons fournir de "preuve» au sens classique du terme, d'au moins essayer de transmettre comment nous avons découvert ce que nous avons trouvé. Ce point-là est en débats et chaque accompagnement nouveau d'un mémoire ou d'une thèse nous permet de remettre à l'épreuve la réflexion à ce propos.

Pour ma part, je fais l'hypothèse que, sans doute, du fait que nous ne sommes pas assujettis aux contraintes, normes, habitudes, jurisprudences instaurées par la discipline psychologie à laquelle appartient la psychologie clinique, nous bénéficions d'une beaucoup plus grande marge de manœuvre que nos collègues psychologues. Au fond, pour moi, je me reconnais dans une filiation qui est celle du courant Pédagogie et psychanalyse, transformée aujourd'hui en Psychanalyse et éducation (2005), et qui, comme nous le montrons dans notre note de synthèse (2005), à la suite de celle de Jean-Claude Filloux publié en 1987, nous fait remonter jusqu'à Freud et Ferenczi, sans passer par la psychologie qui, elle, comme on le sait, a opéré une «difficile rencontre» avec la psychanalyse, pour ne pas dire «impossible» selon les termes propres d'Annick Ohayon dans son travail de 1999, réédité en 2006 aux Éditions La Découverte.

Claudine BLANCHARD-LAVILLE

Équipe Savoirs et rapport au savoir du CREF

Université de Nanterre - Paris $X$

\section{Références bibliographiques}

Ardoino J. (200o), Les avatars de l'éducation, Paris, PUF.

BeIllerot J. (1987), Savoir et rapport au savoir. Disposition intime et grammaire sociale, Note de soutenance, Thèse de doctorat d'État sous la direction de G. Vigarello, Université René Descartes - Paris V.

16. Devereux 1967. 
Beillerot J. (1996), "Désir, désir de savoir, désir d'apprendre», in Pour une clinique du rapport au savoir, J. Beillerot, C. Blanchard-Laville, N. Mosconi (dir.), Paris, L'Harmattan, p. 51-74.

BeILLEROT J. (2000), «Le rapport au savoir», in Formes et formations du rapport au savoir, N. Mosconi, J. Beillerot, C. Blanchard-Laville (dir.), Paris, L'Harmattan, p. 303-317.

Blanchard-Laville C. (coord.) (1999), «Recherches cliniques d'inspiration psychanalytique en éducation et formation ", Revue française de pédagogie, n’ 127.

Blanchard-Laville C. (2001), Les enseignants entre plaisir et souffrance, Paris, PUF.

Blanchard-LAville C. (2003), "Rapport au savoir et approche clinique des pratiques enseignantes», in Rapport au savoir et didactiques, M. Sylvette, M. Caillot (dir.), Paris, Fabert.

Blanchard-Laville C. (2005), "Travail enseignant (aspects cliniques)», in Dictionnaire encyclopédique de l'éducation et de la formation, P. Champy, C. Étévé (dir.), Paris, Retz, p. 1002-1007.

Blanchard-Laville C. (2006), "Psychanalyse et enseignement», in Traité des sciences et pratiques de l'éducation, J. Beillerot, N. Mosconi (dir.), Paris, Dunod.

Blanchard-Laville C. (2007), «Du soin psychique aux enseignants. Psychopathologie du quotidien de l'enseignant», Cliniques méditerranéennes, $\mathrm{n}^{\circ}$ 77, p. 159-176.

Blanchard-Laville C., Chaussecourte P., Hatchuel F., Pechberty B. (2005), «Recherches cliniques d'orientation psychanalytique dans le champ de l'éducation et de la formation ", Revue française de pédagogie, n 151, p. 111-162.

Bourguignon O., Bydlowski M. (dir.) (1995), La recherche clinique en psychopathologie. Perspectives critiques, Paris, PUF [chapitres 1-2, «Le processus de recherche» et 1-3, «Le chercheur, vocation, engagement, formation»].

CASTARÈDE M.-F. (1989), «L'entretien clinique à visée de recherche», in L'entretien clinique, C. Chaland (dir.), Paris, PUF.

Chaussecourte P. (2003), Observations cliniques en sciences de l'éducation. Microanalyses et observations directes de pratiques d'enseignant(e)s de mathématiques, Thèse de doctorat en sciences de l'éducation, Université Paris X.

Chaussecourte P. (2006), «Controverses autour de l'observation des nourrissons", Perspectives Psy, vol. 45, n’ 3 , p. 274-288.

Cifali M. (1999), «Clinique et écriture. Un apport de la psychanalyse aux sciences de l'éducation », Raisons éducatives, $\mathrm{n}^{\circ}$ 1, p. 293-313.

Devereux G. (1967), De l'angoisse à la méthode dans les sciences du comportement, Paris, Flammarion.

FILloux J.-C. (1987), « Psychanalyse et pédagogie ou: d'une prise en compte de l'inconscient dans le champ pédagogique", Revue française de pédagogie, n’ 81.

Ghiglione R., Richard J.-F. (1993), Cours de psychologie. II, Paris, Dunod (chapitre «Définition de la recherche clinique»). 
Ohayon A. (1999), L’impossible rencontre. Psychologie et psychanalyse en France (1919-1969), Paris, La Découverte.

Pechberty B. (2003), «Apports actuels de la psychanalyse à l'éducation et l'enseignement: un éclairage fécond», Études de linguistique appliquée, n 131, p. 265-273.

Pechberty B. (2003), «La difficulté d'apprendre et d'enseigner entre soin et formation », note de synthèse pour l'habilitation à diriger des recherches, Université Paris X, Nanterre.

Revault d'Allones C. (dir.) (1989), La démarche clinique en sciences humaines, Paris, Dunod.

Revault D'Allones C. (1998), «L'étude de cas: problèmes déontologiques et éthiques au cœur d'une méthode», Psychologie clinique, vol. 5, p. 51-62.

SÉchaud E. (dir.) (1999), Psychologie clinique. Approche psychanalytique, Paris, Dunod.

YeLNiCK C. (2005), "L'entretien clinique de recherche en sciences de l'éducation ", Recherche et formation, $\mathrm{n}^{\circ} 50$, INRP. 



\section{LES RECHERCHES EN SCIENCES DE L'ÉDUCATION ET DANS LES DISCIPLINES ÉPONYMES}

- La philosophie - 



\section{DE LA NAISSANCE DES PHILOSOPHES DE L'ÉDUCATION EN FRANCE}

Résumé: Comment sont nés les philosophes de l'éducation en France? Pour répondre à cette question, cinq aspects vont être développés: il n'y a pas de philosophes de l'éducation chez les philosophes; la science de l'éducation est le fait de philosophes qui s'oublient; le moment où Joseph Leif inscrit les philosophes comme formateurs privilégiés des maitres du primaire; l'institutionnalisation des sciences de l'éducation provoque la naissance des philosophes de l'éducation; aujourd'hui, installée, la philosophie de l'éducation est parcourue de tensions constitutives et dynamisantes.

Mots clés: philosophie de l'éducation, histoire des disciplines, sciences de l'éducation, histoire de l'éducation, épistémologie.

Qu'il me soit permis de saluer ce colloque de Caen en commençant par souligner que le temps n'est plus où l'université de Paris s'indignait en 1433 de la naissance de l'université de Caen (cf. "L'université de Paris proteste contre la fondation de celle de Caen - 12 novembre $1433{ }^{1}{ }^{1}$ ).

Ce temps n'est plus, notamment grâce aux sciences de l'éducation, puisque c'est ensemble que les universités de Paris et de Caen (avec celle de Bordeaux) ont créé les cursus de sciences de l'éducation en 1967.

On n'oubliera pas non plus que les sciences de l'éducation de Caen ont déjà organisé un premier colloque en octobre 1988 autour de la question / affirmation «Sciences de l'éducation. Sciences majeures». On sentait bien que, sous l'affirmation «Les sciences de l'éducation sont désormais majeures », pointait la question «Le sont-elles vraiment, au regard des autres disciplines universitaires dont elles sont issues?». Je suis d'ailleurs un peu effrayé de prendre la parole aujourd'hui, puisque, sur les vingtcinq conférenciers qui ont participé aux actes de ce colloque (Issy-les-Moulineaux, Editions EAP, 1991), seuls trois d'entre eux sont convoqués aujourd'hui: Gaston Mialaret, Jean-Louis Martinand, Jean Houssaye - et j'en suis. Je ne sais comment interpréter ce fait...

1. Cité par Riché \& Verger 2006, 246-247. 
Je me demande d'ailleurs si le complexe des sciences de l'éducation vis-à-vis des autres sections universitaires, que l'on pouvait supputer dans le titre de ce premier colloque et que l'on pourrait considérer comme le complexe de l'enfant naturel (ou du bâtard) face à la famille légitime (et à sa noblesse), ne se retrouve pas dans le titre de ce second colloque: la comparaison / justification de la recherche en sciences de l'éducation au regard des «disciplines éponymes ", c'est-à-dire des disciplines qui donnent leur nom aux sciences de l'éducation... La sociologie, la philosophie, la psychologie, l'histoire nous donneraient donc notre nom, à nous sciences de l'éducation... Voilà qui mérite réflexion! En tout état de cause, les sciences de l'éducation existent institutionnellement depuis quarante ans et, après s'être longtemps interrogées sur leur légitimité, leur nature et leur spécificité, les voici qui se demandent si leurs recherches, institutionnellement spécifiques, le sont épistémologiquement. Bon courage! On pourra toujours se dire que la question est légitimée par le fait que la faculté de psychologie et de sciences de l'éducation de Genève se l'est déjà posée en $2004^{2}$. Mais, pour ma part, je ne considérerai cette question avec sérieux et avec un clin d'œil que le jour où les sociologues de la section sociologie, les psychologues de la section psychologie et les historiens de la section histoire se poseront une telle question. Je pense que ce jour n'est pas en vue.

Je vais donc prendre mon courage à deux mains pour envisager une telle question. Seulement, en tant que devant me saisir de la philosophie, j'ai un sérieux avantage sur mes collègues qui doivent s'emparer de l'histoire, de la psychologie et de la sociologie. Et c'est ce que je vais essayer de vous montrer en abordant le problème sous l'angle suivant, sachant qu'il est préférable d'avoir à se poser la question du nonêtre à partir de l'être plutôt que celle de l'être à partir du non-être: d'où viennent les philosophes de l'éducation? Je vais le faire à travers cinq affirmations.

\section{Il n'y a pas de philosophe de l'éducation chez les philosophes}

Si, depuis des siècles, il y a de la philosophie de l'éducation, il n'y a pas à proprement parler de philosophes de l'éducation. Certes des philosophes, depuis l'Antiquité, traitent de l'éducation, mais l'éducation n'est, au mieux, qu'un de leurs objets de réflexion. On peut même assurer que c'est un de leurs objets secondaires. Pour s'en assurer, on pourrait regarder les différents auteurs cités par les ouvrages de base qui présentent la philosophie de l'éducation ${ }^{3}$ pour repérer les philosophes de référence en éducation. Nous nous baserons d'abord sur l'ouvrage qui a servi pendant longtemps d'introduction au domaine, La philosophie de l'éducation d'Olivier Reboul (1989). Qui présente-t-il comme "classiques» dans sa bibliographie? Platon, Aristote, SaintAugustin, Locke, Rousseau, Kant, Fichte, Nietzsche, Comte, Alain et Dewey. Qui peut

2. Chatelanat et al. 2004.

3. Drouin-Hans 1998; Morandi 2000; Kerlan 2003, etc. 
prétendre que, chez ces philosophes, l'éducation soit la question centrale et la plus développée de leur philosophie? Certains pourraient peut-être être tentés de mettre Rousseau à part. Certes il a bien dit que Émile ou De l'éducation (1762) était son ouvrage le plus important. Faut-il le croire pour autant et, surtout, faut-il considérer que, sur l'ensemble de ses écrits, ce livre, essentiel et capital certes, notamment au regard de son influence dans le champ éducatif, représente dans son œuvre la part la plus volumineuse? Je peux, au moins, en douter. On pourrait tenir le même raisonnement à partir de l'ouvrage coordonné par P. Kahn, A. Ouzoulias et P. Thierry sur L'éducation. Approches philosophiques (1990), qui vont rajouter d'autres philosophes au catalogue (Montaigne, Descartes, Condorcet, Hegel, Marx, Arendt, Bergson, Merleau-Ponty), sans que l'on puisse infirmer mon propos.

On peut même aller plus loin et prétendre que les philosophes ne sont pas des philosophes de l'éducation parce qu'ils sont devenus professeurs, au lieu de rester pédagogues. On peut interpréter ainsi ce que dit Pierre Hadot, référence de l'histoire de la philosophie antique, sur les premiers professeurs de philosophie. Dès l'Antiquité, la profession de professeur se structure sur cette tension entre les tenants d'une philosophie conçue avant tout comme exercice dialectique et comme discours théorique et éloquent, et les tenants d'une philosophie conçue avant tout comme mode de vie. Cette tension est un combat. Et ce combat engendrera un vainqueur : l'institution. La profession de professeur va de plus en plus s'instituer et de moins en moins convaincre. Professeurs de l'enseignement fonctionnarisé, les professeurs de philosophie vont avoir comme fonction de professer «l'histoire» de la philosophie, de donner «des renseignements» sur la philosophie.

Ne reste-t-il rien de la conviction, de la vie selon les idées? Si, de façon minoritaire. En effet, à côté de cet enseignement fonctionnarisé, subsistent des écoles privées, destinées avant tout à ceux qui se convertissent, par un choix personnel, à la vie philosophique. C'est là que se pratiquent encore la communauté de vie et l'ascèse philosophique. Les professeurs de conviction vont donc subsister, attachés à leurs idées dans la mesure où elles témoignent d'un mode de vie. Ce sont des éducateurs pour lesquels la question de l'éducation est bel et bien vitale. Et on va pouvoir les considérer comme à l'origine de la lignée des pédagogues qui, eux, vont faire de l'éducation l'objet central de leurs pratiques et de leurs idées.

Retenons donc que, tout au long de l'histoire de la philosophie, l'éducation est restée un objet mineur. Et il en est de même au $\mathrm{XX}^{\mathrm{e}}$ siècle. Des philosophes continuent à s'intéresser aussi à l'éducation, sans en faire leur objet premier. Les hommes de foi semblent même en faire une de leurs obligations, si l'on considère la Théorie de l'éducation de Laberthonnière (1935) ou Pour une philosophie de l'éducation de Maritain (1959). Et aujourd'hui ? Si l'on veut bien dépasser la bulle inflationniste des philosophes républicains antipédagogues ${ }^{4}$ et si l'on veut bien considérer que des philosophes traitant de l'éducation en poste en philosophie ont souvent été obligés

4. Bourgeois 1984; Kintzler 1984; Finkielkraut 1987; Debray 1991; Coutel 1991; Baudart 1991; Péna-Ruiz 1991 et 2005; Muglioni 1993; Michéa 1999; Jaffro \& Rauzy 1999, etc. 
d'obtenir leur qualification dans la section de sciences de l'éducation du CNU, on ne repère que peu de noms s'intéressant aux questions éducatives, sans jamais en faire leur objet principal: Gauchet, Kambouchner, Rancière, Renaut, Serres et VieillardBaron ${ }^{5}$ peuvent être cités dans cette catégorie. Il n'y a donc pas, en philosophie, de philosophe de l'éducation, ne serait-ce que parce qu'il n'y a pas de poste défini ainsi (alors que la philosophie des sciences, l'esthétique, l'éthique et bien d'autres spécifications fleurissent). Terminons ce premier point en soulignant une exception: il y a peut-être eu un philosophe de l'éducation en philosophie, c'était Jacques Ulmann. Professeur de philosophie à Paris I, non seulement sa célèbre thèse (La nature et l'éducation, 1964) est bien consacrée à l'éducation, mais l'essentiel de ses publications ultérieures l'est aussi. Saluons cette exception.

\section{La science de l'éducation est le fait de philosophes qui s'oublient}

Après bien d'autres ${ }^{6}, \mathrm{P} . \mathrm{Kahn}^{7}$ rappelle très justement que, à la fin du XIX ${ }^{\mathrm{e}}$ siècle, le discours pédagogique était républicain et mobilisait les représentants en vue de l'institution philosophique. L'école républicaine de Jules Ferry a non seulement institutionnalisé la pédagogie (publication du Dictionnaire de pédagogie; création du Musée pédagogique; fondation de la Revue pédagogique), mais elle a aussi universitarisé cette même pédagogie en créant un premier cours de science de l'éducation à la Sorbonne fin 1883 pris en charge par Marion, provoquant ainsi une généralisation de ce cours dans les facultés des lettres de province et attribuant systématiquement ces cours à des professeurs de philosophie. Ces cours pouvaient s'appeler pédagogie, science de l'éducation ou psychologie appliquée à la pédagogie (en écho, dans le dernier cas, aux nouveaux programmes des Écoles normales d'instituteurs de 1881). Voici donc les philosophes installés comme spécialistes de l'éducation au nom d'une science de l'éducation qui s'impose dans le cadre d'une formation professionnelle des enseignants.

Certes quelques philosophes à ce moment vont résister à cette affirmation d'une possibilité et d'une nécessité d'une connaissance rationnelle, transmissible, applicable en éducation. Ils ne feront souvent que reprendre le leitmotiv des spécialistes des disciplines. Quel est-il ? On peut le symboliser par le propos de Brunetière, un académicien, homme de lettres et professeur à l'École normale supérieure, que Daniel Hameline a si bien analysé ${ }^{8}$ : «Ayons avant tout des professeurs qui ne songent qu'à professer, et moquons-nous de la pédagogie!» (Éducation et instruction,

5. Gauchet 1985; Kambouchner 2000; Vieillard-Baron 1994; Rancière 1987; Renaut 2004; Serres 1992; Vieillard-Baron 1994.

6. Charbonnel 1988; Fabre 2002; Gautherin 2002; Houssaye 1991; Houssaye \& Hameline 2002.

7. Kahn 2006.

8. Hameline 1993 . 
1895, p. 8). En effet, à la fin du XIX ${ }^{\mathrm{e}}$ siècle, la pédagogie fait aussi rire, la pédagogie fait aussi peur. Elle fait rire par son côté démodé, dépassé et prétentieux. Elle fait peur parce qu'elle porte en elle une menace de faux-savoir qui dissoudrait la culture. Il est donc normal, logique, nécessaire et salutaire de nier la pédagogie, disent ses adversaires. Parce qu'elle n'existe pas en tant que telle, parce qu'elle n'est en aucune façon spécifique.

Il reste que, dans les années 1880 , ce sont des philosophes qui vont se poser en spécialistes de la pédagogie. Ce sont des philosophes qui réduisent la pédagogie à la psychologie de l'éducation, et ce dans le giron de la philosophie, alors que la psychologie va au même moment commencer à s'autonomiser (c'est en 1889 qu'est fondé à l'École des hautes études à Paris le «laboratoire de psychologie»). Ils ont pour nom Compayré, Pécaut, Gréard, Marion, Buisson, Thamin, Dumesnil, Janet. Malgré les nuances, la tentative dominante sera celle-ci: réduire la pédagogie à une science de l'éducation en tant que psychologie appliquée, au sein de la philosophie. Telle est la conception qui va devenir prégnante avant le tournant du siècle, à tel point que la psychologie appliquée à l'éducation sera mise au programme des Écoles normales primaires et supérieures en 1881. Ce n'est qu'en 1920 que la sociologie s'ajoutera au programme, mais cette fois la philosophie sera souterrainement menacée, car la sociologie prétend, elle aussi, révéler les fins de l'éducation. Notons cependant que ces philosophes ne produisent pas de philosophie de l'éducation à proprement parler, ils ne sont pas des philosophes de l'éducation, mais des professeurs de pédagogie ou de science de l'éducation.

Quoi qu'il en soit, la pédagogie devient science parce qu'elle se résout, se dissout en savoir psychologique appliqué. La philosophie classique nourrira la psychologie expérimentale et en fait la pédagogie expérimentale de Binet, Claparède, Buyse ou Aurélien Fabre. Grâce à la philosophie, la pédagogie meurt, la science puis les sciences de l'éducation naissent. Durkheim avait eu beau, dès 1911, prévenir que la pédagogie était une théorie pratique, rien n'y a fait; la pédagogie a été réduite à une pratique d'application d'une théorie scientifique externe. Il est vrai que Durkheim aura plus que tendance à opérer la même démarche, au profit, cette fois, de la sociologie.

Bref, il apparaît que, pour les philosophes en éducation de 1880, la science de l'éducation est donnée comme l'apogée de la philosophie de l'éducation par le truchement de la psychologie scientifique. Il semble bien cependant que ceci soit resté plus une intention qu'une réalité, si l'on suit les analyses de Gautherin. Celle-ci a montré que la science de l'éducation universitaire est restée une science principalement spéculative, éloignée de toute préoccupation empirique. Les professeurs de science de l'éducation se sont posés en théoriciens et non en experts de l'éducation. Ils produisaient des théories générales de l'éducation à qui on reprochait l'inutilité de leur pédagogie. Ils remplirent leur mission: «justifier une politique». Professeurs de science de l'éducation et de pédagogie, les philosophes ont ainsi pu s'inscrire institutionnellement comme les spécialistes de la pédagogie, au nom de la psychologie, sans se vouloir pour autant philosophes de l'éducation à proprement parler. 


\section{Le moment où Joseph Leif inscrit les philosophes comme formateurs privilégiés des maîtres du primaire}

C'est donc tout à fait légitimement (c'est-à-dire en cultivant ces ambiguïtés), si l'on peut dire, que les professeurs de philosophie sont devenus en 1947 des professeurs de psychopédagogie chargés d'enseigner dans les Écoles normales à la fois la pédagogie générale, la philosophie de l'éducation, la psychologie de l'enfant et l'anthropologie sociale. Seulement ce baptême de possession reconnue de la pédagogie va se lézarder peu après: la psychologie va quitter les eaux de la philosophie et se constituer en secteur indépendant qui, certes, ne récuse pas ses origines, mais qui tient tout de même à mettre plus que des distances. La psychologie de l'enfant s'épanouit, accompagnée dans une moindre mesure par la psychologie de l'éducation.

Que devient la pédagogie dans tout cela? Va-t-elle passer corps et biens à la psychologie, au nom de la science, et échapper ainsi à la philosophie? Pas du tout. La philosophie, en tant qu'institution, va continuer à se donner comme le référent de la pédagogie, elle va accaparer la pédagogie et la réduire à elle. Pour preuve, on se reportera à la réforme introduite par Joseph Leif en 1969 (soit deux ans après la création des premiers cursus de sciences de l'éducation) dans les Écoles normales; on confiera de nouveau aux philosophes la pédagogie générale et on retrouvera la tradition de l'histoire des doctrines de l'éducation. La psychologie n'est pas absente, mais elle participe d'un ensemble plus vaste qui fait de la philosophie de l'éducation la spécialiste des généralités et qui continue à justifier la place des philosophes dans les lieux de formation pédagogique initiale. Quand les généralités deviendront à leur tour vraiment trop générales et quand surtout il apparaîtra que les savoirs de sciences de l'éducation se réfèrent de plus en plus à des disciplines typées, reconnues et indépendantes (psychologie, sociologie, histoire, économie, etc.), les généralités s’affineront et la philosophie de l'éducation (en tant que contenu) se repliera sur un noyau dur, qu'elle nommera le plus souvent étude des buts et des fins de ces moyens qui constituent la pédagogie. Moyen de dire que l'essentiel, le sens, doit présider à la mise en œuvre, même si par ailleurs ces moyens pédagogiques vont continuer à aller chercher des justifications dans les sciences humaines. N'est-ce pas encore un moyen de tenir les sciences, même si on n'assimile plus la philosophie aux sciences? Le principal, c'est que la pédagogie, elle, reste sous contrôle, mais cette fois elle a deux mentors, la philosophie d'une part, les sciences de l'éducation d'autre part, ces dernières ayant été induites par la première. Autrement dit, les philosophes s'installent comme les spécialistes de la formation des maîtres du primaire autour de la psychopédagogie. Ce qui est d'ailleurs très frappant, c'est que, pendant longtemps, alors que les philosophes sont nombreux sur ces postes dans les Écoles normales, ils ne produiront pratiquement rien en philosophie de l'éducation.

En fait, on ne sait pas grand chose sur cette période et concernant ces philosophes. On peut rêver d'une thèse sur le sujet. Et notamment d'une thèse sur celui qui me semble au cœur de cette inscription des philosophes dans la poursuite de la tradition républicaine pour les considérer avant tout comme des spécialistes de 
la pédagogie, Joseph Leif, qui a été philosophe, inspecteur de philosophie, inspecteur d'académie avant d'être inspecteur général de l'instruction publique. Comment conçoit-il ces philosophes? Comme des professeurs de pédagogie. Le sont-ils encore au nom de la science de l'éducation? On peut en douter, d'autant que les sciences de l'éducation existent désormais à l'université. Qu'est-ce que Leif attend des philosophes en École normale? Certainement de changer la pédagogie des maîtres dans un sens rénovateur.

Leif lui-même est un novateur engagé. Il participe fortement à la réforme des collèges de 1963 où l'on voit Jean Capelle créer les collèges d'enseignement secondaire avec, notamment, les classes de transition pour les élèves de sixième qui auraient trop de difficultés à suivre un cycle moderne ou classique. Joseph Leif sera, avec Maurice Rouchette, l'un des deux inspecteurs généraux qui piloteront la rénovation pédagogique de l'école primaire à partir de 1964. Leif semble avoir trois cordes à son arc. En premier lieu, il produit de nombreux manuels scolaires, toujours en duo, pour le collège, chez Nathan et Delagrave. Il se fait une spécialité des manuels d'instruction civique (la commune en 1962 avec Rustin; le département en 1962 avec Rustin; l'organisation économique et sociale en 1963 avec Richard; la vie administrative et l'effort français en 1963 avec Grimal; la vie politique en 1963 avec Joxe; la France et l'Europe dans le monde en 1965 avec Grimal; l'initiation à la vie active en 1970 avec Valot; les travaux pratiques en 1977 avec Taguel, etc.). Mais il faut aussi ajouter des manuels de calcul, de leçons de choses et de sciences (1958 avec Dézaly), d'histoire et géographie (1957 avec Rustin), de français (1962 avec Vérel), etc. Bref, on peut dire que notre inspecteur tient une place considérable dans la production des manuels scolaires, principalement en sixième et en cinquième.

La deuxième corde à son arc se nomme psychologie. Leif y fait des incursions, notamment en proposant en 1976, avec un adepte de la non-directivité, Lucien Brunelle, Le jeu pour le jeu, après avoir publié à partir de 1965 Psychologie et éducation en quatre tomes (L'enfant; L'adolescent; La psychométrie; Les textes) (avec Delay, puis Juif). Mais l'aspect le plus intéressant pour nous est le troisième aspect, soit la pédagogie. Leif, là encore, y règne en maître, au moins sur le plan de l'édition. Outre quatre ouvrages de Pédagogie spéciale (1957) consacrés en fait aux contenus disciplinaires et un ouvrage intitulé Esprit et évolution des civilisations en 1950, notre inspecteur publie une Histoire des institutions scolaires en 1964 (avec Rustin), une Morale professionnelle à l'usage des instituteurs et des institutrices en 1966 (toujours avec Rustin), une Pédagogie générale par l'étude des doctrines pédagogiques en 1954 et 1965 (encore avec Rustin) et enfin Les doctrines pédagogiques par les textes en 1966 (avec Biancheri). Il poursuit en 1978 par Qu'est-ce que la rénovation pédagogique?, en 1979 par la Formation des enseignants, en 1980 par Problèmes de psycho-pédagogie, avant de titrer en 1970 (puis en 1999) une Philosophie de l'éducation en quatre tomes qui est en fait la reprise de ses ouvrages antérieurs (Pédagogie générale; Inspirations et tendances; Doctrines pédagogiques).

De ce catalogue tout à fait impressionnant, ne retenons que trois choses autour des ouvrages de pédagogie générale. En premier, Leif conforte les professeurs de 
philosophie en tant que professeurs de pédagogie dans les Écoles normales. En deuxième, il fournit à ces professeurs des manuels de pédagogie, qui répondent en fait point par point au programme des Écoles normales publié le 15 novembre 1947, notamment sur l'histoire des doctrines pédagogiques. En troisième, tardivement, cette pédagogie générale va être baptisée philosophie de l'éducation, sans que rien ne change fondamentalement. Sur le plan des contenus, Leif dispense, d'une part, une histoire des idées éducatives de l'Antiquité au $\mathrm{XX}^{\mathrm{e}}$ siècle (sous la rubrique «sens de l'éducation ») et, d'autre part, une présentation des méthodes pédagogiques axée sur la supériorité des méthodes actives. Il conforte ainsi la tradition qui institue les philosophes en tant que professeurs rénovateurs de pédagogie pour les maîtres du primaire. Il ne suscite pas à proprement parler de philosophes de l'éducation, mais il maintient le réservoir de ceux qui devront ensuite le devenir, quand les véritables philosophes de l'éducation seront effectivement apparus.

\section{L'institutionnalisation des sciences de l'éducation provoque la naissance des philosophes de l'éducation}

C'est donc en 1967 que débarquent les trois Pilgrim Fathers des sciences de l'éducation: Jean Chateau à Bordeaux, Maurice Debesse à la Sorbonne et Gaston Mialaret à Caen instituent les sciences de l'éducation. D'où viennent-ils? De la psychologie tous les trois, d'une psychologie qui se veut expérimentale, tournée vers la pédagogie, proche de l'éducation nouvelle. Que pensent-ils de la philosophie? Sans doute qu'il est nécessaire de la dépasser pour parvenir à une connaissance rationnelle, scientifique et fondée. Que pensent-ils de la pédagogie? Sans doute que le temps est venu de l'arracher aux généralités philosophiques pour l'arrimer aux connaissances démontrées de la recherche en psychologie. Rappelons cette première phrase écrite par Jean Chateau, qui se présente comme «Professeur de pédagogie à la Faculté des Lettres de Bordeaux", dans le premier article du premier numéro de la Revue française de pédagogie:

Toujours nous nous heurtons au manque de critères solides. La seule pédagogie reste ici impuissante... Il nous faut donc nous tourner vers la psychologie... elle seule peut fournir les bases d'une pédagogie valable... Sans suffisante psychologie, je ne peux qu'errer au hasard... Psychologie d'abord, pédagogie ensuite, telle est donc la règle logique ${ }^{9}$.

Quoi qu'il en soit, une fois passés en sciences de l'éducation, nos fondateurs vont se considérer fort logiquement comme des psychologues de l'éducation. Et ils vont accueillir, pour renforcer leurs équipes d'enseignants à l'université, des philosophes qui, eux, vont pouvoir alors se dire philosophes de l'éducation. On voit ainsi

9. Chateau 1967,10 . 
apparaître des philosophes patentés de l'éducation qui n'auront plus à se dire pédagogues (d'autant que les psychologues, eux, veulent contrôler la pédagogie). Des philosophes qui vont devoir faire de la philosophie de l'éducation. Des spécialistes en sciences de l'éducation dont l'objet central est bien l'éducation, mais à partir d'une démarche philosophique qui saura d'ailleurs tout de suite s'inscrire contre le «tout scientifique» des pères fondateurs.

Au trio des pères fondateurs des sciences de l'éducation, on peut ainsi ajouter le quatuor des premiers philosophes de l'éducation en titre. Il s'agit de Georges Snyders à la Sorbonne, d'Olivier Reboul à Strasbourg, de Guy Avanzini à Lyon et de Daniel Hameline à l'Institut supérieur de pédagogie de Paris. Leurs histoires ne sont pas semblables bien entendu, mais ils vont représenter la première vague des philosophes de l'éducation, capables même de créer les sciences de l'éducation dans leur université (Avanzini à Lyon et Reboul à Strasbourg). Le plus emblématique d'entre eux est sans doute Snyders, dans la mesure où sa thèse, dès 1965, est consacrée à la pédagogie des XVII ${ }^{e}$ et XVIII ${ }^{e}$ siècles et où il participera très activement à la vie des sciences de l'éducation. Curieusement, il commencera par enseigner la psychologie de l'enfant à la création de la licence de psychologie à Nancy, avant d'être recruté à la Sorbonne par Debesse (contre Ulmann d'ailleurs) pour y enseigner la psychologie en sciences de l'éducation puis la philosophie. Reboul, après sa thèse sur Alain, aura l'audace, de son côté, de faire fonctionner son poste de philosophie principalement en sciences de l'éducation, poste de philosophie de l'éducation qui sera ensuite perdu au profit de la philosophie du langage. Quant à Avanzini et Hameline, ils seront bien sur une optique philosophique, tout en gardant pendant un certain temps des attaches du côté de la psychologie (cf. leurs thèses: sur Binet pour Avanzini et sur le rapport au savoir d'un point de vue psychanalytique pour Hameline).

Ce quatuor se verra rejoint au fur et à mesure par d'autres philosophes de l'éducation en poste en sciences de l'éducation: Fumat à Montpellier, Hannoun à Dijon, Natanson à Rouen, Soëtard à Lille. On peut aussi y adjoindre Ardoino à Caen et sans doute bien d'autres. Qu'est-ce qui les caractérise? Ils se réclament de la philosophie et de ses références historiques; autrement dit, ils ont à cœur de montrer qu'ils connaissent leurs classiques. Pour autant, s'ils donnent des gages à la philosophie classique de l'éducation, ils se comportent comme des modernes, des contemporains, et non comme des historiens de la philosophie ou de la philosophie de l'éducation ou des doctrines pédagogiques. Par contre, ils prennent en compte les autres disciplines des sciences de l'éducation (psychologie, psychanalyse, sociologie, histoire), soit en tant qu'objets de réflexion, soit dans leurs propres démarches de chercheurs. Ils n'hésitent pas à nourrir les débats sur la spécificité et l'épistémologie des sciences de l'éducation. Ce sont avant tout des philosophes qui se veulent professeurs de sciences de l'éducation. Voilà, les philosophes de l'éducation sont nés et installés en sciences de l'éducation. Ils vont pouvoir notamment accueillir le réservoir des philosophes en École normale, enfants et petits-enfants de Leif, pour leur permettre de faire des thèses de philosophie de l'éducation, lorsque ces derniers soit vont estimer que cela leur serait intellectuellement profitable, soit vont comprendre qu'ils ne peuvent pas faire 
l'économie institutionnelle de la thèse (à la création des IUFM plus particulièrement). Une étude serait à mener sur ces professeurs de philosophie en École normale, pour saisir leur évolution sur ce point (sachant que les uns se sont tournés vers des thèses de philosophie, d'autres vers des thèses en sciences de l'éducation, sans pour autant qu'il s'agisse de philosophie de l'éducation).

\section{Aujourd'hui, installée, la philosophie de l'éducation est parcourue de tensions}

Actuellement, le nombre de ceux qui se réclament de la philosophie de l'éducation est impressionnant. La liste des publications établie par Anne-Marie Drouin en témoigne suffisamment. Qui plus est, le milieu s'organise autour de colloques, de revues (Penser l'éducation, Le Télémaque) et d'associations (AECSE de façon globale et SOFPHIED de manière spécifique). Pour autant, si l'on regarde ceux qui se revendiquent de la philosophie de l'éducation et leurs publications, il faut bien dire que la définition est large, sinon lâche. L'épuration est certes impossible à concevoir, même si on constate un certain affadissement de la définition par rapport aux fondateurs. En même temps on voit bien que, pour certains, il suffit d'avoir fait de la philosophie pour estimer qu'on le reste, même si on est devenu psychologue, sociologue ou historien. Quoi qu'il en soit, institutionnellement, les philosophes de l'éducation en France relèvent soit des départements universitaires de sciences de l'éducation soit des IUFM. Il ne serait pas faux d'avancer que la domination des départements est réelle. Il suffit de considérer le bureau et le comité fondateur de l'association SOFPHIED pour s'en persuader. Il y aurait là à la fois une logique du respect de la naissance des philosophes de l'éducation et un renversement symbolique de la tradition des philosophes professeurs de pédagogie. Il est bien entendu difficile d'avancer des éléments fiables sur les philosophes de l'éducation aujourd'hui. Je ne veux, pour ma part, qu'esquisser quelques tensions qui parcourent le champ.

La première est inscrite dans l'histoire des philosophes de l'éducation. Sont-ils d'abord philosophes ou d'abord en sciences de l'éducation? Ne seraient-ils pas mieux en philosophie, tout compte fait? N'est-ce pas leur milieu naturel, qui pourrait leur pardonner leur dérive institutionnelle? La consécration ne serait-elle pas dans la reconnaissance des «vrais " philosophes? Après tout, ils ont bien un objet justificatif historique donné, la tradition philosophique en éducation, appuyée sur la déférence de la philosophie générale des philosophes.

La deuxième tourne autour de la pédagogie. Les philosophes ne peuvent plus se prétendre spécialistes de la pédagogie. D'autant que les sciences de l'éducation d'une part se sont emparées de l'objet (au besoin pour le réduire) et que la philosophie d'autre part s'ingénie à en faire son repoussoir (au besoin dans la caricature). Il est donc devenu difficile de se dire à la fois pédagogue et philosophe. Et pourtant, ne serait-ce qu'en raison du long compagnonnage entre les deux domaines, les philosophes de l'éducation ne peuvent récuser cet ancrage pédagogique et sa réalité. 
La troisième tension relève d'un écartèlement de fait dans la production des savoirs. Le philosophe de l'éducation est tributaire d'un côté du secteur de la philosophie en tant que lieu de production renouvelée des savoirs philosophiques et d'un autre côté du secteur des sciences de l'éducation en tant que lieu de production effectif des savoirs de philosophie de l'éducation. Condamné au nomadisme, le philosophe de l'éducation se voudrait parfois sédentaire.

La quatrième vient de la différence entre les savoirs élaborés par les philosophes de l'éducation d'une part et les savoirs des autres disciplines classiques des sciences de l'éducation, disciplines qui, elles, de plus, peuvent se dire vraiment scientifiques et qui ne vont pas manquer de se poser comme justifiées et dominantes, et d'être ressenties comme hégémoniques et suffisantes.

La cinquième s'adresse plus spécifiquement à l'histoire. Une des traditions de la philosophie de l'éducation (et on a vu historiquement pourquoi) a toujours été l'histoire des conceptions pédagogiques. Il est donc logique que bien des philosophes de l'éducation se soient empressés de s'inscrire dans cette tradition. Seulement les règles du jeu tendent à se modifier, d'une part parce que les règles de la méthode historique s'imposent de plus en plus, d'autre part parce que l'objet histoire des idées pédagogiques est fortement revendiqué par des historiens patentés (à l'intérieur ou à l'extérieur des sciences de l'éducation).

La sixième tension est épistémologiquement plus lourde. Les positivistes en sciences de l'éducation continuent à voir dans la philosophie et sa démarche en sciences de l'éducation un reste malheureux à éliminer, ne serait-ce que pour garantir l'image de scientificité de la section sciences de l'éducation. En réponse, les philosophes de l'éducation se donnent aussi comme rôle de diminuer et de relativiser l'aspect «science» dans la démarche et le contenu des recherches et des savoirs en éducation.

La septième tient à la composition interne des philosophes de l'éducation. La plupart des philosophes de l'éducation ne sont pas purs, au sens où ils sont philosophes et autre chose: psychologues, historiens des idées, épistémologues, pédagogues... Le philosophe de l'éducation est un penseur «biface». Cet aspect, en même temps, lui donne une crédibilité (il a un « objet»), comme si la pensée «pure» en éducation risquait d'être trop éthérée et peu crédible. En même temps, cet aspect biface le menace dans la reconnaissance de son être (ce n'est pas un vrai philosophe...). Tant et si bien qu'on se demande si chacune de ses faces est un hobby ou une essence. C'est aussi ce qui autorise indûment les non-philosophes ou les anciens philosophes, devenus historiens, psychologues ou sociologues, à estimer qu'ils peuvent prétendre être encore philosophes en éducation.

Faut-il s'alarmer outre mesure de ces tensions? Non, puisqu'elles sont constitutives de la philosophie de l'éducation et de la philosophie de l'éducation en sciences de l'éducation. D'une certaine manière, ces tensions nourrissent l'existence et la vitalité de la philosophie de l'éducation. Qui s'en plaindrait? Certainement pas moi. Je suis heureux de constater que la vie de la philosophie de l'éducation est bel et bien au sein des sciences de l'éducation (au moins de facto, sinon de jure). Et je plains beaucoup mes collègues historiens, psychologues et sociologues de devoir 
se poser ainsi tragiquement la question du rapport avec leur discipline éponyme, car ils vivent toujours sous la menace de devoir estimer que le «vrai » savoir légitime se construit en dehors d'eux. Les philosophes de l'éducation, eux, sont beaucoup moins en mesure d'être complexés: n'ayant pas de véritable concurrence, ils n'ont qu'à défendre le statut de la philosophie de l'éducation, aussi bien en interne, par rapport aux autres domaines des sciences de l'éducation, qu'en externe par rapport à la philosophie.

Juste retour des choses et clin d'œil de l'histoire: ce sont des philosophes qui ont généré la, puis les sciences de l'éducation; et ce sont les sciences de l'éducation qui, à leur tour, ont généré les philosophes de l'éducation. J'ai donc essayé de vous montrer que les philosophes de l'éducation sont nés véritablement à la naissance des sciences de l'éducation. La première génération, celle de la création, a pris sa retraite dans les années 1990. La seconde génération, celle de l'extension, s'est retirée officiellement dans les années 2000. La troisième génération, la mienne, celle de l'organisation et de l'installation, constituera les retraités des années 2010. Il reste à souhaiter réussite et approfondissement à la quatrième génération. De manière provocatrice, je dirai, pour terminer, que les philosophes de l'éducation seront pleinement accomplis en sciences de l'éducation le jour où, sur un poste de philosophie de l'éducation, on recrutera une personne qui aura fait tout son cursus en sciences de l'éducation, sans être passée par celui de la philosophie. Alors les sciences de l'éducation seront devenues des sciences majeures, comme annoncé dans le premier colloque de Caen en octobre 1988. Mais, dans ce cas, la question des rapports avec les disciplines éponymes aura-t-elle encore un sens?

Jean Houssaye

CIVIIC

Université de Rouen

\section{Références bibliographiques}

BAudart A. (1991), «La scholè ou les dimanches de l'esprit», in Les préaux de la République, A. Baudart, H. Péna-Ruiz (coord.), Paris, Minerve.

Bourgeois B. (1984), «L'école sans sa République?», in Philosophie, école, même combat, Paris, PUF.

Charbonnel N. (1988), Pour une critique de la raison éducative, Berne, Peter Lang.

Chateau J. (1967), «Pour une éducation scientifique», Revue française de pédagogie, $\mathrm{n}^{\circ} 1$.

Chatelanat G., Moro C., SaAda-Robert M. (dir.) (2004), Unité et pluralité des sciences de l'éducation. Sondages au cour de la recherche, Berne, Peter Lang. 
Coutel C. (dir.) (1991), La République et l'École. Une anthologie, Paris, Presses Pocket.

Debray R. (1991), "Éloge de nos maîtres», in Les préaux de la République, A. Baudart, H. Péna-Ruiz (coord.), Paris, Minerve, p. 13-24.

Drouin-Hans A.-M. (1998), L'éducation, une question philosophique, Paris, Anthropos.

FABRE M. (2002), « Les controverses françaises sur l'école: la schizophrénie républicaine», in Enseigner et libérer, C. Gohier (dir.), Québec, Les Presses de l'université Laval.

Finkielkraut A. (1987), La défaite de la pensée, Paris, Gallimard.

Gauchet M. (1985), « L'école à l'école d'elle-même», Le Débat, n’ 37.

GAUtherin J. (2002), Une discipline pour la République. La science de l'éducation en France (1882-1914), Berne, Peter Lang.

Hameline D. (1993), «L'école, le professeur et le militant», in La pédagogie, une encyclopédie pour aujourd'hui, J. Houssaye (dir.), Paris, ESF.

Houssaye J. (1991), «Les 105 ans des sciences de l'éducation: fin de la pédagogie?», in Sciences de l'éducation. Sciences majeures, Issy-les-Moulineaux, EAP.

Houssaye J. (1999), Éducation et philosophie. Approches contemporaines, Paris, ESF.

Houssaye J., Hameline D., Soëtard M., Fabre M. (2002), Manifeste pour les pédagogues, Issy-les-Moulineaux, ESF.

Jaffro L., Rauzy J.-B. (1999), L'école désouvrée. La nouvelle querelle scolaire, Paris, Flammarion.

KAHN P. (2006), «La critique du "pédagogisme” ou l'invention du discours de l'autre», Les sciences de l'éducation. Pour l'ère nouvelle, vol. 39, n 4.

Kahn P. (2006), «La philosophie de l'éducation au miroir de l'histoire», Le Télémaque, n' 30, p. 131-136.

Kahn P., Ouzoulias A., Thierry P. (1990), L'éducation. Approches philosophiques, Paris, PUF.

Kambouchner D. (2000), Une école contre l'autre, Paris, PUF.

Kerlan A. (2003), Philosophie pour l'éducation, Issy-les-Moulineaux, ESF.

Kintzler C. (1984), Condorcet, l'instruction publique et la naissance du citoyen, Paris, Gallimard.

LABerthonnière L. (1935), Théorie de l'éducation, Paris, Vrin.

Leif J., Rustin G. (1953), Pédagogie générale par l'étude des doctrines pédagogiques, Paris, Delagrave.

LeIf J., Rustin G. (1954), Histoire des institutions scolaires, Paris, Delagrave.

Maritain J. (1959), Pour une philosophie de l'éducation, Paris, Fayard.

MichÉA J.-C. (1999), L'enseignement de l'ignorance et ses conditions modernes, Castelnaule-Lez, Climats. 
Morandi F. (200o), Philosophie de l'éducation, Paris, Nathan.

Muglioni J. (1993), L'école ou le loisir de penser, Paris, CNDP.

PÉNA-Ruiz H. (1991), «Les faux-semblants du droit à la différence», in Les préaux de la République, A. Baudart, H. Péna-Ruiz (coord.) Paris, Minerve.

PÉna-Ruiz H. (2005), Qu'est-ce que l'école?, Paris, Gallimard.

RANCIÈre J. (1987), Le maître ignorant. Cinq leçons sur l'émancipation intellectuelle, Paris, Fayard.

Reboul O. (1989), La philosophie de l'éducation, Paris, PUF.

Renault A. (2004), La fin de l'autorité, Paris, Flammarion.

Riché P., Verger J. (2006), Des nains sur les épaules des géants, Paris, Tallandier.

Serres M. (1992), Le tiers instruit, Paris, Gallimard.

Ulmann J. (1964), La nature et l'éducation, Paris, Vrin.

Vieillard-Baron J.-L. (1994), Qu'est-ce que l'éducation? Montaigne, Fichte et Lavelle, Paris, Vrin. 


\section{DE LA RECHERCHE EN PHILOSOPHIE DE L'ÉDUCATION}

Résumé: Les recherches entreprises en philosophie de l'éducation ne bénéficient pas d'un contexte particulièrement favorable du fait de la prégnance de concepts et thématiques empruntés à la philosophie politique, lesquels sont destinés à alimenter certaines thèses d'un débat sur l'école. Notre souci sera de montrer qu'à rebours la philosophie de l'éducation permet, dans son moment critique, de dépasser les alternatives mythiques que ces thèses ont construites, et d'ouvrir dans l'horizon ainsi dégagé une approche spécifique de l'éducation. Deux perspectives critiques sont ainsi présentées: la perspective déconstructive telle que Jacques Derrida l'avait ouverte en faisant de la question éducative une question philosophique centrale, celle de l'herméneutique philosophique fondée par Gadamer expressément autour de la question de la Bildung. À travers ces perspectives, la philosophie de l'éducation retrouve la dynamique d'une philosophie sociale telle que Rousseau l'avait inaugurée, et peut entreprendre un dialogue fructueux avec les disciplines des sciences de l'éducation, en particulier, dans cette communication, en interrogeant le thème de la différance chez Derrida.

Mots clés: philosophie de l'éducation, déconstruction, herméneutique, générativité.

\section{Introduction: une certaine façon de chercher}

«Fait-on de la recherche de la même façon en philosophie et en philosophie de l'éducation?» demandent les organisateurs de ces journées. C'est sur une certaine façon de chercher qu'il faut donc d'abord se concentrer. «Façon» est une métaphore dont Descartes se sert peu, sauf à y être contraint dans la jonction entre le concept intellectuel et la sensation, entre l'esprit et le corps: "Accoutumés à ne rien considérer qu'en s'imaginant, qui est une "façon" de penser particulière pour les choses matérielles ${ }^{1}$.

Descartes, par-delà l'universalité du penser, distingue donc des modes de ce penser, suivant l'objet vers lequel il s'oriente. Il nomme cela «façon", en ayant recours à une métaphore derrière laquelle il y a «faire» - et la résistance de la matière, comme

1. Descartes, Discours de la méthode, IV, 6. 
dans «manière», son synonyme, il y a la main, qui appartient au corps. Mais l'objet auquel s'applique cette «façon" est effectivement matériel. C'est pourquoi la question qui demande si l'on recherche de la même façon en philosophie comme en philosophie de l'éducation nous entraîne, d'emblée, sur le terrain de quelque chose qui résiste, et avec lequel il faut savoir s'y prendre. Savoir s'y prendre, savoir y faire avec les choses, c'est prendre en compte un objet résistant, qui fait problème, parce que, comme la matérialité vis-à-vis du penser chez Descartes, il nous installe dans la présence - c'est le rôle essentiel de l'éducation, même si nous refusons qu'il soit notre présence - nous ne sommes pas le produit de notre éducation.

Il y aurait donc quelque chose de l'éducation qui résisterait à une inspection transparente de l'esprit et qui donnerait à la "philosophie de l'éducation" son statut de discipline propre, parce que la «façon» d'y chercher l'en sépare quelque peu - non pas de la philosophie, mais d'autres façons de pratiquer la philosophie.

Mais que signifie cette hypothèse selon laquelle la philosophie de l'éducation a affaire à une résistance propre du réel qui se fait jour dans l'éducation?

Elle a d'entrée de jeu une signification épistémologique qui impose deux conséquences:

- la première est que la philosophie de l'éducation n'est pas elle-même une région de la philosophie générale, une philosophie appliquée ou une recherche limitée à certains objets;

- la seconde conséquence est que la philosophie de l'éducation entretient des relations de coopération avec les sciences objectives de l'éducation, et ne prétend en aucune manière, ignorer ou mépriser les savoirs construits dans ces disciplines; au contraire, elle s'en instruit pour sa propre recherche.

Suivant la première conséquence, qui serait celle de l'isonomia, du partage égal du pouvoir, la philosophie de l'éducation a une égale compétence pour chercher à éclairer les questions ontologiques, métaphysiques et éthiques. Selon la seconde, qui serait celle des isopsèphoi, de "ceux qui sont égaux dans le vote», la philosophie de l'éducation donne sa voix dans le débat sur les questions éducatives et peut se placer légitimement, comme on l'illustrera, sur le terrain empirique.

\section{De la recherche en philosophie de l'éducation}

\section{Les embarras d'une recherche}

Si la philosophie recherche bien dans la direction de cette résistance, il faut cependant commencer par comprendre comment cette recherche a pu être entreprise. Or, il faut l'admettre sans ambiguïté: cette situation n'est pas, depuis nombre d'années, spécialement favorable à la philosophie de l'éducation. Cela ne tient pas à une conjoncture contingente, ou à des influences individuelles négatives. La raison en est plutôt à rechercher dans ce que Jacques Derrida présente de la structure de la philosophie: 
la philosophie est irremplaçable, écrivait-il à l'occasion de la réforme Giscard-Haby, parce qu'elle se remplace elle-même toujours: «le combat n'est jamais pour ou contre la philosophie, sa présence ou son absence, mais entre des forces et leurs instances philosophiques, au-dedans et au dehors de l'institution scolaire $»^{2}$. En tant qu'elle implique un rapport à l'État et à l'histoire, l'éducation est une question qu'il a semblé à certaines instances dangereux d'ouvrir à la recherche philosophique, dans la mesure où toutes les questions y auraient été clarifiées, et les problèmes résolus. Il n'y a plus rien à chercher, soit que l'histoire est close et son sens devenu patent, soit que cet apogée du sens est déjà derrière nous, et qu'il suffit de retourner sur ses pas pour retrouver le moment historiquement exceptionnel de la fusion de l'État et de son projet éducatif.

C'est ce qui fait que la recherche en philosophie de l'éducation a été mise dans l'embarras par la prégnance de concepts de la philosophie politique dans le champ de l'éducation, sous la forme que l'on connaît du «républicanisme dogmatique», comme Michel Fabre l'a montré. Et l'arme privilégiée, dans l'ordre du discours, pour engager le combat a été la convocation de deux mythes, transformés pour qu'ils deviennent antagonistes.

Pourquoi la pensée moderne produit-elle des mythes, et principalement dans le champ éducatif? La philosophie des Lumières s'est déterminée comme une philosophie de l'éducation en promettant à la fois d'émanciper l'individu de ce qui le tient en minorité: les passions, les opinions et les liens particuliers qui l'enserrent dans un monde borné, et aussi de conduire l'humanité tout entière vers un état dans lequel sa rationalité lui aurait permis de mettre fin aux malheurs qui l'accablent et dont elle s'avère seule responsable. À l'espérance eschatologique promise par les autorités théologico-politiques succède un projet pour l'humanité d'assumer la responsabilité du monde ${ }^{3}$. La question éducative repasse ainsi au premier plan. L' «éducation du genre humain » se dédouble dans une articulation du particulier au général selon laquelle l'individu doit être éduqué pour que l'humanité elle-même s'éduque, mais l'éducation de l'individu n'est possible que dans une humanité elle-même en marche vers l'éducation ${ }^{4}$. La temporalité de l'attente eschatologique se sécularise alors en une historicité dans laquelle l'humanité construit son présent par son activité propre et peut en prévoir son avenir comme conséquence de cette action ${ }^{5}$.

Or cette prévision du futur est une promesse d'un salut advenant à l'humanité par le progrès qui résultera nécessairement de son orientation vers la rationalité. Cette prévision, montre Koselleck ${ }^{6}$, est la production d'une utopie, dont la fonction de mythe est de rassembler une communauté dans la promesse de l'attente. Et s'il y a un terreau qui s'avère d'une extrême fertilité pour la levée des utopies politiques, c'est celui de l'expérience éducative.

\footnotetext{
2. Derrida 1975, 458.

3. Revault d'Allonnes 2006.

4. Lessing 1976.

5. Löwith 2002.

6. Koselleck 2005.
} 
Or cette expérience ne peut être lue, pour l'idéalisme, qu'à travers le concept d'identité, identité du subjectif et de l'objectif, de la nature et de l'esprit. Éduquer l'humanité, en vue de former l'individu, instruire l'individu, en vue de former une humanité nouvelle, tels sont les termes dans lesquels les pédagogues de la Révolution française poseront le problème de l'éducation, en articulant l'éducation des adultes et l'instruction des enfants. Ce n'est donc pas principalement la raison qu'il s'agit de former dans l'humanité nouvelle, et les révolutionnaires comprennent qu'il s'agit plutôt de dégager progressivement le peuple que l'on destine à former une nation, de son passé de ténèbres et d'assujettissement. Il convient, pour ce faire, de s'adresser à sa faculté de sentir plutôt qu'à son entendement, à son cœur et à son imagination. Baczko montre ainsi que l'éducation du peuple va être pensée à travers un projet politique de fêtes civiques dans lesquelles se rassemble une communauté qui se façonne elle-même par le sentiment d'appartenance commune aux mythes révolutionnaires et à leurs "grands récits " ${ }^{7}$. Mais si l'on éduque les adultes, l'on instruira les enfants, en vue de leur éducation citoyenne future; la différence entre éduquer et instruire se fonde alors sur une opposition entre spontanéité et liberté, entre instant présent et futur, entre imagination et raison, entre plaisir et mortification. La pensée révolutionnaire façonne deux utopies éducatives, celui d'une éducation par la communion dans la fête, dont les linéaments sont empruntés à Rousseau, et celui d'une école close instruisant les enfants en vue de les orienter vers la rationalité. Ces utopies sont solidaires, ainsi les projets révolutionnaires prévoient-ils que chaque fête civique s'organisera autour d'un noyau d'instruction publique, assuré par l'instituteur et ses classes. La distinction entre éduquer et instruire n'est donc pas, à l'origine, une question politique, c'est une question philosophique à laquelle se heurte l'action politique comme problème à résoudre.

\section{Construction d'un antagonisme}

Or la pensée républicaine, par un jeu de glissements et de déplacements, va entreprendre de les rendre, comme on le sait, antagonistes. Mais pourquoi? Notre hypothèse est que la pensée républicaine ouvre le combat pour le compte de la métaphysique de l'État moderne, d'une immanence voulant se présenter comme transcendance fondée dans l'apriorisme de la raison. Cette hypothèse est étayée par les catégories métahistoriques de Koselleck. En distinguant en effet le champ d'expérience et l'horizon d'attente comme structures de notre expérience historique, Koselleck présente deux figures de la modernité: le républicanisme et le démocratisme. La thèse de Koselleck est que «les Temps modernes ne se saisissent comme des temps nouveaux que depuis le moment où les attentes se sont éloignées de toutes les expériences faites jusqu'alors ${ }^{8}$. Or cette tension croissante entre l'expérience et l'attente se trouve investie par des énoncés concurrentiels tendant à la rendre supportable. Réciproquement, le

7. Baczko 1982.

8. Ibid., 315 . 
champ sémantique politique et social se modèle sur cette tension inouie entre expérience et attente. Comme le dit Koselleck, "plus l'expérience est mince, plus l'attente est grande», et plus vite vont s'user les anciennes attentes au contact des nouvelles expériences. Le républicanisme, analyse Koselleck, est ce concept de mouvement qui permet de produire des énoncés porteurs d'une promesse de réalisation de la notion de progrès dans l'action politique, alors que le démocratisme soutient la même thèse dans le champ de l'action sociale et donc éducative. Les deux sont une anticipation du mouvement de l'histoire par la transformation de la pratique que le républicanisme veut voir résulter de la puissance de l'État politique, et que le démocratisme - réticent vis-à-vis de l'autoritarisme de l'État, confie bien plutôt à la vie sociale. La pensée politique du néo-républicanisme en France s'orientera vers le champ éducatif lorsqu'elle comprendra que le démocratisme ouvre l'école aux enjeux sociaux et aux attentes de la société vis-à-vis de sa demande de justice distributive. Les républicains dogmatiques transforment alors les utopies solidaires en mythes antagonistes.

Quels sont ces mythes? Le premier est celui d'une école républicaine dont on sait, grâce aux études critiques entreprises en histoire et en philosophie de l'éducation, qu'elle n'a jamais possédé les vertus qui lui ont été prêtées. Le second mythe, moins exploré pour des raisons sur lesquelles il faudrait se pencher, est celui d'une pédagogie apophatique, beaucoup plus négative encore que celle de l'Émile, puisqu'elle ne s'orienterait que sur les désirs immédiats ou socialement médiatisés des élèves. Il faudrait aussi montrer rigoureusement qu'une telle pédagogie n'a jamais existé empiriquement, parce qu'elle serait autoréfutative dans la pratique. Opposant les deux mythes, le républicanisme extrait l'école de la société, afin de la protéger, dit-il, d'une mort inéluctable. Les termes du débat sont connus.

La conséquence majeure de cette structuration du champ éducatif a été d'engager la réflexion dans une controverse sans issue où toute recherche critique se trouvait immédiatement balayée par une perspective hypercritique la dénonçant comme participant d'un complot conduisant l'école à son déclin.

\section{La perspective critique de la philosophie de l'éducation}

Comment dépasser l'aporie? Il est nécessaire d'échapper à l'idéalisme en refusant le rêve d'une synthèse finale de l'identité de l'esprit et du monde, réalisable par l'éducation, d'abandonner les mythes qui soutiennent le combat idéologique que nous avons décrit. En un mot, abandonner le comme si pour le comme tel, et revenir à la philosophie. C'est une perspective critique, au sens où Alain Vergnioux la distingue parmi les orientations structurelles de la philosophie de l'éducation ${ }^{9}$. Or cette perspective, si elle désire se préserver contre l'hypercritique prescriptive, peut, avec profit, s'orienter selon deux axes, qu'il importe d'expliciter: celui de la déconstruction des concepts importés dans le champ éducatif, et celui de l'herméneutique de l'expérience éducative. C'est sur eux que les recherches peuvent s'engager.

9. Vergnioux 2006. 


\section{La métaphysique de la présence}

Un des rôles traditionnels dont la philosophie de l'éducation s'est trouvée investie depuis son institutionnalisation au sein des sciences de l'éducation a été «d'éclairer les antinomies" propres à l'acte d'éduquer: nature et culture, liberté et contrainte, spontanéité et réceptivité, raison et passions, etc. ${ }^{10}$, bref de poursuivre la tâche de l'idéalisme transcendantal. Or le commentaire infini des oppositions métaphysiques traditionnelles ne pouvait que renforcer l'image d'une philosophie de l'éducation, spécialisée dans la reprise des questions générales appliquées à des objets spécifiques. Il ne s'agissait, au mieux, que d'aider les autres disciplines des sciences de l'éducation à bénéficier des avancées conceptuelles réalisées en philosophie générale, au pis à leur donner une légitimité grâce au travail de préparation que la philosophie de l'éducation avait réalisé.

Une rupture était donc nécessaire; elle devait rompre avec les restaurations postkantiennes de la métaphysique, comme avec la vision purement politique de la question éducative. Le penseur qui à la fois s'attaqua aux couples de la métaphysique et remit au premier plan la question éducative comme question philosophique, c'est Jacques Derrida. Le problème que posa très tôt Derrida fut de comprendre comment s'institue la présence dont la métaphysique considère qu'elle est toujours déjà-là, dans la plénitude du Logos ou de la Raison qui permet à l'esprit de s'emparer de cette présence. Ce que la métaphysique ne peut pas penser, c'est le processus d'inscription dans la présence, ce qui, dans l'éducation par exemple, fait apparaître un sujet éducable, qui ne préexiste pas à ce qui l'éduque. Derrida analyse ainsi le privilège absolu que la métaphysique accorde à la voix, sur les traces et sur l'écriture: la voix installe dans l'écoute, et lorsqu'il s'agit de la parole divine, la présence de l'homme ne peut trouver son sens que dans cette écoute, même si ce sens tarde à se manifester, puisqu'il y a promesse, pour celui qui écoute, qu'un tel sens se dévoile à la fin. Ce privilège aboutit à une disqualification de l'écriture et des inscriptions de traces, comme "mauvaise présence», puisqu'elles ne rendent présent que dans leur propre effacement. Ainsi le paradigme même de l'éducation selon la métaphysique consiste-t-il bien dans cette préparation à l'écoute de la parole vraie, au-delà de ce qui la brouille. On comprend aussi pourquoi la figure de Rousseau sera, pour Derrida, emblématique d'une rupture avec cette tradition.

Ce privilège de la toute-présence permet alors d'installer les couples conceptuels de la Nature et de l'Esprit, de l'Animalité et de l'Humanité, de la Liberté et de la Nécessité, à travers lesquels la philosophie pense son autre, ce qui la limite et qu'elle entend maîtriser: la présence moindre, qu'elle se réapproprie dans un processus de dévalorisation et que Derrida explore dans Marges. Aussi, interprétant la Destruktion de Heidegger, Derrida ouvre-t-il la voie à la déconstruction, dont l'enjeu est d'analyser ce que la métaphysique cache de son propre fonctionnement: la formation des

10. Mialaret 1967,82 . 
antinomies, l'élaboration des concepts. La déconstruction selon Derrida aboutit à démonter les structures de la métaphysique, à repérer la genèse des concepts fondateurs, à vider de leur prétendu contenu d'être les substantialisations classiques. Ce qui favorisera la discussion avec certains courants de la philosophie analytique américaine. Or, précisera Derrida, ce qui résiste à la déconstruction, c'est la justice. Nous reviendrons sur ce point.

\section{Les couples conceptuels en éducation}

Si l'on veut bien examiner les antinomies classiques de la philosophie de l'éducation, il est possible de s'apercevoir qu'elles trouvent leur racine dans ce privilège de la présence. C'est une direction de la recherche déconstructive en éducation, dont on peut donner un aperçu rapide. La pédagogie de la modernité est habitée par ce primat de la présence immédiate: celle naturelle, donc moindre, de l'enfant qui doit être convertie, selon le terme de Durkheim, vers la certitude de la conscience rationnelle, présence pleine de l'humain. Ou a contrario, chez Rousseau, par la vérité de la présence enfantine, corrompue dans cet âge adulte qui est la moindre présence de l'essence de l'homme, par l'opacité des signes sociaux et le privilège dont jouit l'apparence sur l'être.

Mais comme le montre Derrida, Rousseau ne distribue pas le partage sans hésitation, en montrant que la vérité de l'enfant est aussi la première manifestation de la déficience qui, dans l'ordre de la nature, appelle la suppléance. L'éducation se construit sur cette finitude, ce qui rend sa tâche paradoxale : la pédagogie doit intervenir le moins possible et le plus tard possible, mais elle doit intervenir. La finitude humaine, telle qu'elle se dévoile dans l'enfance, est ce paradoxe d'une présence qui ne peut accéder à sa pleine manifestation qu'en courant le risque de s'aliéner en s'exposant à ce qui la limite: autrui, les signes de la culture. Mais loin de lui, les mythes fonctionnent sans inquiétude: la culture contre la nature, la vérité de l'instant contre le mensonge d'un futur aliénant. Tous les débats sur l'éducation en France depuis les années soixante s'y sont alimentés, faute sans doute de s'ouvrir à cette thématique rousseauiste de l'« exposition», que les pédagogues allemands comme Georg Picht et Hartmut von Hentig, eux, ont pris en considération.

\section{L'éducation comme différance}

Derrida ouvre cependant cette perspective de l'exposition grâce à la notion de différance. La différance est une notion qui, par sa forme passive-active, permet de comprendre l'éducation comme un acte d'institution, et non plus un processus de conversion dans lequel l'élève cesse d'être momentanément sujet pour devenir objet, ou réciproquement, redevient sujet pour avoir été pris trop longtemps comme objet, sujet rebelle à la violence de l'institution. L'éducation ne se fait pas à partir d'une présence pleine, mais elle est l'institution, sans achèvement peut-être, d'une présence. Il n'y a pas de présence pleine du maître qui, comme le voudrait Levinas, soit à elle 
seule instituante de l'élève ${ }^{11}$. Il n'y a pas davantage de présence pleine de l'enfant qui, comme dans le mythe de l'enfant naturel, soit la base constitutive de l'acte éducatif. En effet la différance est instituante parce qu'elle dépasse le présent et s'expose comme un procès de temporisation: c'est l'intervalle entre passé et présent, entre présent et futur qui est marqué par l'éducation; l'éducation temporise. Elle le fait d'abord en inscrivant le temps mort dans le temps du vivant, par l'instruction et la transmission des traces du passé devenu savoir ${ }^{12}$. L'éducation, comme toute écriture, est d'origine testamentaire: origine elle-même sans origine, puisqu'indéfiniment recommencée. Instruire n'est pas contraindre une subjectivité privée, c'est arracher le présent à son illusion solipsiste pour le relier à l'humanité. L'éducation temporise - deuxième temps - en tournant le présent vers le futur : c'est l'éduquer, si l'on veut maintenir ce couple rhétorique. Le temps mort qui se lève dans le présent (anamnèse, réminiscence du Ménon) rend possible une nouvelle écriture, une inscription de soi dans le futur. Le sujet éducable se laisse alors creuser par son rapport à cet élément futur: pro-jet, formation de soi. Instruire et éduquer sont les deux faces du même procès de la différance qu'inscrit l'éducation, et, de même, toutes les antinomies classiques se trouvent affaiblies. Il est possible alors d'aborder l'acte éducatif à partir de son noyau ontologique propre: comment fait-on être, comment instituet-on dans la présence, à travers l'éducation?

\section{La compréhension d'une résistance}

La perspective de recherche ainsi ouverte permet de comprendre comment l'éducation institue un sujet éducable, pour lequel l'éducation et la formation de soi deviennent le mode fondamental de la présence humaine, d'une présence donc inachevable, et, il faut le dire, sans promesse de salut, ni en Dieu, ni en l'histoire. Elle y parvient par l'enseigner, qui est un art de produire des signes grâce auxquels s'effectue la temporisation: faire signe vers le passé en vue du futur, c'est creuser l'instant présent pour y faire naître l'étonnement, l'inquiétude et le désir d'apprendre; ainsi la pédagogie décrit-elle depuis Platon l'acte d'enseignement. Mais ce désir d'apprendre ne demeurerait qu'une nostalgie d'un passé qui n'a jamais été présent, s'il ne communiquait pas un pouvoir sur le monde que les signes représentent. L'enseignement institue certes l'altérité de l'élève de manière radicale, comme le pense Levinas, mais dans un jeu de traces, hors toute origine absolue: le passé vers lequel tend le désir d'apprendre ne sera jamais présent, il sera toujours autre que ce que les signes voulaient représenter; l'enseignement n'est pas un modelage ou une conformation selon un archétype, une origine absolue. C'est pourquoi la temporisation du futur fait de l'élève un auteur, qui peut alors s'autoriser à porter un nom. Derrida pense que la signature du nom propre est le propre de l'enseignement. Paul Ricœur n'est pas éloigné de cette

11. Levinas 2006 .

12. Derrida 1967. 
perspective lorsqu'il thématise la question du parcours de la reconnaissance, comme attestation donnée au sujet de ses capacités d'agir, par le passage du passif à l'actif ${ }^{13}$. Enseigner, c'est distribuer des signes par lesquels le sujet se reconnaît comme appartenant à un monde, culturel et épistémologique, et s'y reconnaît comme acteur de ses projets. C'est à ce titre qu'il s'y connaît.

\section{La question de la philosophie sociale}

L'approche déconstructive permet de dépasser les apories traditionnelles et de libérer la philosophie de l'éducation des assujettissements que lui imposent les disciplines qu'elle inquiète, dans la mesure où elle met en question, d'entrée de jeu, toutes les assurances positivistes. Mais toute déconstruction est entreprise, non pas pour le bénéfice exclusif de la philosophie, mais en vue de la pratique. La déconstruction derridienne ouvre la possibilité de la philosophie de l'éducation en tant que philosophie sociale, engagée dans la pratique par l'interprétation de l'expérience éducative. Axel Honneth définit la philosophie sociale comme une spécificité de l'aire germanique, indépendante de la philosophie politique, et dont la tâche est de définir et d'analyser les processus d'évolution de la société qui sont des «pathologies du social» ${ }^{14}$. Le fondateur de la philosophie sociale, pour Honneth, est Rousseau, dès le Discours sur les sciences et les arts, lorsqu'il fait le diagnostic de la modernité en termes de pathologie sociale, relativement aux critères éthiques que les concepts d'opacité et d'aliénation permettent de définir. C'est dans cet ordre du questionnement que la philosophie de l'éducation peut rencontrer concrètement les sciences de l'éducation du point de vue critique, tout en échappant au dogmatisme d'une certaine pensée politique ${ }^{15}$. La véritable sensibilité aux pathologies sociales donne en effet la priorité au fait éducatif, comme Rousseau en avait montré la voie. Or l'analyse de l'expérience éducative ne peut s'entreprendre que dans l'orientation propre à cette sensibilité, qui est une ouverture à la fragilité de l'autre. C'est ainsi que le second axe sur lequel la philosophie de l'éducation tend à déployer sa perspective critique est celui de l'herméneutique $^{16}$.

\section{De la certitude de soi à la compréhension de soi}

Certes la perspective herméneutique semble devenir une référence de plus en plus partagée, grâce à la réception grandissante de l'œuvre de Paul Ricœur. Néanmoins il convient de la distinguer rigoureusement de la compréhension à l'œuvre dans les

\footnotetext{
13. Ricœur 2004.

14. Honneth 2005.

15. Dans cette perspective, qu'il nous soit permis de renvoyer à nos propres travaux sur la construction de l'éthique professionnelle des enseignants, ouverts par la thèse réalisée sous la direction de Marguerite Altet en 2003 à l'université de Nantes.

16. C'est ce que confirmèrent sans ambiguïté les rencontres de la Sofphied à Paris, en juin 2007.
} 
sciences humaines, comme dans la sociologie de Weber. L'herméneutique philosophique ne commence véritablement qu'à partir de l'instant où l'on fait de la compréhension la structure même de l'existence de l'homme ${ }^{17}$ et que l'on thématise cette compréhension comme le fait premier du langage: si la structure de l'existence humaine réside dans l'effort pour comprendre le monde et autrui, et se comprendre à partir de cet effort, il apparaît que la compréhension ne saurait excéder le langage qui la fonde. L'homme échappe à l'autoréférence d'une compréhension centrée sur soi-même par la frange d'incompréhension même qu'y introduit le langage qui, nous aidant à comprendre, obscurcit autant qu'il dévoile. Ce statut du langage dans l'herméneutique est ce qui interdit de réinstaller le sujet classique de la métaphysique, maîtrisant le monde dans ses représentations.

C'est la voie qui sera choisie par Gadamer ${ }^{18}$. Et ce qui est remarquable est que cette perspective, comme celle de la déconstruction, rencontre la question de la Bildung comme la question centrale de la compréhension de soi et du monde. Une herméneutique philosophique doit être, si elle prétend être à la hauteur de son projet, une philosophie de l'éducation. Mieux encore, il est possible de discerner, dans toute l'histoire de l'herméneutique, un enjeu éducatif secret qui nous incite à penser que ce n'est pas l'éducation qui rencontrerait, par un phénomène de mode intellectuelle vain et éphémère, l'herméneutique, mais bien la question de la présence de l'homme en formation de soi-même à travers la parole des autres, qui taraude l'herméneutique.

L'éducation est pensée par Gadamer comme le partage du sens à travers le dialogue indéfini de la culture. C'est sur ce point que la déconstruction et l'herméneutique se séparent. Pour l'herméneutique de Gadamer, en effet, l'éducation manifeste une résistance du sens, que la déconstruction derridienne entend détruire, comme reliquat métaphysique de l'unité. Cette résistance est celle de la cohésion des unités de vie qui se maintiennent dans les interactions humaines, ces unités culturelles qui se transmettent dans l'histoire, explique Gadamer. Le paradigme en est le dialogue socratique, dans lequel il est nécessaire de passer par un autre pour comprendre et accéder à la connaissance: "nous pensons avec autrui, pour nous retourner vers soi comme un autre», dit Gadamer. C'est pourquoi, «comprendre, c'est être pris ».

Du point de vue de la critique du dogmatisme philosophique en éducation, l'herméneutique de Gadamer ruine ce postulat métaphysique de la certitude de soi qui fondait le sujet cartésien. Ce qui mine toute certitude de cet ordre est la limitation de l'expérience humaine, qui n'est traduisible que dans la communication langagière. Or cette communication est frappée d'une double finitude: celle du caractère chancelant des significations et celle plus radicale encore que Schleiermacher avait mis en évidence dans le phénomène universel de la mécompréhension : toute communication présuppose et se nourrit de mécompréhension, qui paradoxalement soutient l'effort pour

17. Heidegger 1986.

18. Gadamer 1976. 
comprendre et maintient l'opacité éthiquement nécessaire d'autrui ${ }^{19}$. Or l'expérience éducative est le dévoilement le plus manifeste de la structure dialogique de l'existence. Être en éducation ou en formation signifie être en projet et ne pas être un sujet installé dans une présence pleine, ni d'ailleurs dans un processus vers cette présence qui n'est qu'un leurre, car chaque projet se transforme par le dialogue qu'il permet d'entretenir avec le monde. Selon Gadamer, «le dialogue que nous sommes n'aura jamais de fin", c'est-à-dire que le sujet est toujours en compréhension de soi, et ne parvient jamais à l'intuition immédiate de quelque chose qui serait «sa vérité».

Mais cette compréhension de soi ne peut passer que par autrui, et l'éducation peut être saisie alors comme cette mise en route vers la compréhension de soi, dans un dialogue où autrui nous engage dans la langue, la culture et la connaissance. Le but d'une scolarité réussie n'est-il pas de rendre cette éducation inachevable, en devenant autoformation?

Mais l'inachèvement de l'éducation ne relève pas de l'infini du savoir propre à l'entendement divin, que nous ne ferions qu'apercevoir de très loin; cette sécularisation du savoir resterait encore métaphysique. Le problème est radicalement différent, selon Gadamer. Le fait est que comprendre, c'est comprendre autrement, et que ce factum que l'herméneutique met en évidence pose dans un jour nouveau la question de la transmission.

\section{La transmission du sens}

Transmettre par l'éducation des connaissances, ce n'est pas révéler un sens déjà-là dont l'entendement divin garantirait l'authenticité. C'est la conception que l'on trouve encore, dans sa version sécularisée, chez Durkheim; cette idée d'une grâce qui descendrait sur chaque enfant par sa conversion éducative, et que Blumenberg décrit comme "une singularisation de la raison qui se génère elle-même ${ }^{20}$. Le sens n'est pas une chose présente, un objet accessible dans le monde, le sens est dialogue et ce que transmet l'éducation est un trésor de significations dans lequel l'éduqué forgera des énoncés propres à porter réponse aux questions qu'il sera amené à se poser, du fait même des problèmes que nous lui léguerons pour n’avoir pas su les résoudre.

Mais le savoir ne peut devenir une possession de la vérité que sous la condition que chaque sujet puisse se l'approprier par un travail de connaissance ${ }^{21}$. Rendre ce travail possible est donc une priorité essentielle de l'éducateur, et elle requiert que la réflexion sur le langage s'engage dans une perspective épistémologique qui lui permette de construire des théories analysant les modalités selon lesquelles les énoncés de la transmission deviendront porteurs de sens pour ceux qui sont appelés à les recevoir. Ces théories épistémologiques, qui permettent l'analyse de la production des savoirs

19. Gadamer pose l'authenticité du Mitsein, de l'être avec, et réfute celle du Dasein de Heidegger, comme figure résiduelle du sujet cartésien. L'être avec, et non pas l'être en commun de la philosophie politique.

20. Blumenberg 1999, 84 .

21. Relativement à l'herméneutique de la culture dans l'éducation, cf. Simard 2004. 
ainsi que des systèmes pédagogiques qui les portent, reposent toutes, in fine, sur une théorie de la réception. Théorie double puisqu'elle s'intéresse à la façon dont les élèves comprennent, c'est-à-dire, comprennent différemment les savoirs, mais aussi à la façon dont les enseignants comprennent ou ne comprennent pas pourquoi les élèves comprennent différemment. Une théorie herméneutique de la réception transforme le regard sur la difficulté scolaire: l'élève en souffrance n'est plus une moindre présence devant le vrai, par rapport à celle, rayonnante de l'élève qui réussit, c'est une compréhension de soi qui échoue devant l'opacité de certains énoncés de l'école.

Chaque présence qu'institue l'éducation est marquée du passé et se creuse de la possibilité de l'avenir. Elle est donc, comme le dit Derrida, «hantée » ${ }^{22}$. La position positiviste en éducation, celle qui vise l'immortalité d'une présence pleine, que le mythe nostalgique place du côté de la Troisième République, reposait sur le dogme d'une perfectibilité indéfinie obtenue par une transmission transparente de savoirs sans crises parce que produits par l'accumulation de résultats. Mais hélas nous avons depuis d'autres résultats historiques à interroger, ceux qui ont été produits par un retournement de la raison contre elle-même.

\section{Conclusion : la générativité et les conditions d'un dialogue}

La recherche en philosophie de l'éducation peut revendiquer son autonomie parce qu'elle est d'abord la philosophie qui interroge en direction du réel dans un discours de vérité, et que cette exigence du vrai est ce qui engage à détruire les malentendus et les confusions. Il devient important, pour conclure, d'esquisser les marges de la philosophie de l'éducation, qui lui permettent de dialoguer avec les sciences de l'éducation.

Le problème fondamental de la philosophie de l'éducation, qui devra bien être approfondi un jour, est celui du noyau anthropologique que constitue le fait éducatif. Si ce noyau est fondé sur une essence de l'homme, c'en est fait de tout ce qui précède, et la philosophie de l'éducation peut devenir une facto-anthropologie, et cesser d'être philosophie. Ces tentations existent, et elles sont entreprises souvent de l'extérieur de la philosophie à partir de ses concepts et de ses thématiques.

Formulons le problème: de quoi l'éducation a-t-elle la charge, non plus vis-àvis du mode d'existence de l'homme, cela c'est la condition langagière, mais vis-à-vis de son être-propre, en tant qu'institué par la différenciation? L'éducation, peut-on répondre, a la charge de la générativité. C’est Koselleck qui propose cette catégorie en référence à la fois à la temporalité et à l'historialité de Heidegger. La générativité (Generativität) est proche de la nativité (Gebürtlichkeit) de Hannah Arendt ${ }^{23}$. C'est,

\footnotetext{
22. Derrida 1993.

23. "Le miracle qui sauve le monde, le domaine des affaires humaines, de la ruine normale, "naturelle", c'est finalement le fait de la natalité, dans lequel s'enracine ontologiquement le fait d'agir» (Arendt 1961, 314).
} 
dit-il, «la transformation anthropologique qui, d'un handicap zoologique, fait une réalité humaine». La générativité se traduit empiriquement par l'action des générations dans leur succession diachronique, et elle manifeste cette finitude humaine qui rend possible l'engendrement de nouvelles histoires. Les générations se succèdent en se chevauchant, ce qui constitue le noyau anthropologique de l'éducation. Il y a une nécessité de cette succession, et surpasser cette nécessité a été le rêve obscur de tous les totalitarismes dans l'instauration d'un présent perpétuel. Koselleck montre que «les changements et afflux de générations sont constitutifs des différents horizons historiques finis qui se chevauchent jusqu'à faire advenir les histoires». Ce qui implique que, «étant spécifiques aux générations, les expériences ne sont pas immédiatement transmissibles».

Nous ne pouvons pas comprendre aisément ce qui est proche temporellement de nous, et le caractère essentiel des temps modernes est de rendre cette compréhension encore plus incertaine. Le noyau anthropologique qu'indique Koselleck n'est pas très éloigné - hors tout arrière-plan métaphysique, de la détermination dans laquelle Rousseau pense la finitude humaine à partir de l'enfance. Nous retrouvons ici le point d'articulation dans les sciences de l'éducation entre la philosophie sociale et les autres disciplines. Ainsi l'intérêt d'un tel noyau anthropologique est d'affranchir la philosophie de l'éducation de toute tentation de recourir à une nature humaine pour discuter avec les sciences de l'éducation. Si la générativité n'est pas naturalisable, parce que différance, alors les sciences positives peuvent décrire l'œuvre et les effets de la générativité, tels qu'ils se manifestent dans les relations sociales et dans les processus qui structurent la représentation-compréhension de soi, mais ne peuvent en aucun cas prétendre à remonter à l'origine de la présence elle-même, puisqu'elle se dérobe sans cesse devant toute assignation.

La générativité permet de questionner l'éducation dans les termes de la justice distributive : comment une génération peut-elle produire suffisamment de biens éducatifs pour la génération suivante et en assurer une juste distribution dans le temps de leur chevauchement?

Les conditions d'un dialogue peuvent aisément se déduire de ces prémisses:

- l'abandon de toute velléité prescriptive de la part de la philosophie;

- le travail d'interprétation de l'éducation comme processus d'installation, dont les sciences apportent les signes et les marques;

- l'abandon symétrique, du côté des sciences positives, de toute tentation d'une objectivation dernière de l'éducation: nous ne pouvons pas connaître ce qui nous forme, en nous en extirpant par la réflexion, comme l'avait montré Kant.

Ainsi, ce dialogue entre toutes les disciplines de la recherche en éducation pourrat-il concourir vers un horizon commun, celui d'une école créative en vue d'une distribution juste des biens de l'éducation. Mais au-delà de cette perspective d'une division du travail, au sens où Habermas envisage la coopération entre philosophie et sciences humaines, se dégage un horizon vers lequel seule la philosophie est orientée. C'est celui que Derrida exempte de la déconstruction, celui de la justice, au-delà 
du droit et de la loi, au-delà de la justice distributive et de la politique de l'État. L'éducation est une forme de cette justice, car elle rend justice, en nous permettant de vivre avec les «fantômes» du passé, dans une présence de la mémoire et de l'héritage. En rendant justice à ceux qui nous ont précédés, l'éducation nous permet d'être justes (plus justes) avec les autres, car nous comprenons que notre présence, frangée de l'absence de nos prédécesseurs, ne doit pas réduire l'autre à l'absence ${ }^{24}$. Apprenons à vivre avec les spectres...

Didier MOREAU

CREN

Université de Nantes

\section{Références bibliographiques}

Arendt H. (1961), Condition de l'homme moderne, Paris, Calmann-Lévy.

Васzко B. (1982), Une éducation pour la démocratie. Textes et projets de l'époque révolutionnaire, Paris, Garnier.

Blumenberg H. (1999), La légitimité des Temps modernes, Paris, Gallimard.

Derrida J. (1967), De la grammatologie, Paris, Minuit.

Derrida J. (1975), «Réponses à la Nouvelle Critique», in Qui a peur de la philosophie?, Paris, Flammarion.

Derrida J. (1993), Spectres de Marx, Paris, Galilée.

Gadamer H.G. (1976), Vérité et Méthode, Paris, Seuil.

Heidegger M. (1986), Etre et Temps, Paris, Gallimard.

Honneth A. (2006), La société du mépris, Paris, La Découverte.

Koselleck R. (2005), Le futur passé, Paris, EHESS.

Lessing G.E. (1976), L'éducation du genre humain, Paris, Aubier Montaigne.

Levinas E. (2006), Le temps et l'autre, Paris, PUF.

Löwith K. (2002), Histoire et Salut, Paris, Gallimard.

Mialaret G. (1967), Les sciences de l'éducation, Paris, PUF.

Revault D'Allonnes M. (2006), Le pouvoir des commencements. Essai sur l'autorité, Paris, Seuil.

Ricceur P. (2004), Parcours de la reconnaissance, Paris, Stock.

Simard D. (2004), Éducation et herméneutique. Contribution à une pédagogie de la culture, Québec, Les Presses de l'université Laval.

Vergnioux A. (2006), "Philosophie et éducation», in Traité des sciences et des pratiques de l'éducation, J. Beillerot, N. Mosconi (dir.), Paris, Dunod, p. 489-50o.

24. Derrida 1993, 15. 


\section{TABLE DES MATIÊRES}

\section{OUVERTURE}

Gaston Mialaret : Les origines et l'évolution des sciences de l'éducation en pays francophones.

DE LA PSYCHOPÉDAGOGIE À LA RECHERCHE ÉDUCATIONNELLE

Présentation - Thierry Ріот : Évolution des questions. 25

Marguerite Altet: De la psychopédagogie à l'analyse plurielle des pratiques 31

Gérard Sensevy: Didactique et sciences de l'éducation: une reconfiguration?

\section{NOUVEAUX TERRAINS, NOUVEAUX TERRITOIRES}

Présentation - Jean-François ThÉmines: De nouvelles demandes sociales et de nouvelles problématiques de recherche. . . . . . . . . . . . . . . 61

Vincent LANG : La formation des enseignants. . . . . . . . . . . . . . 65

Dominique FABLET: Le travail social et la formation des travailleurs sociaux 71

LES RECHERCHES EN SCIENCES DE L'ÉDUCATION ET DANS LES DISCIPLINES ÉPONYMES

Présentation - Alain Vergnioux : Maturité et indépendance

La sociologie

Yves DutercQ: Sociologie, sociologie de l'éducation et sciences de l'éducation

Julie Delalande : Pratiquer l'anthropologie de l'enfance en sciences de l'éducation: une aide à la réflexion. 


\section{L'histoire}

Jean-Noël Luc: Territoires et pratiques de l'histoire de l'éducation. Le point de vue d'un historien des $\mathrm{XIX}^{e}$ et $\mathrm{XX}^{e}$ siècles. . . . . . . . . . . . . . . . 115

François JACQUET-FrANCILLON : L'histoire de l'éducation et de l'enseignement dans et hors les sciences de l'éducation . . . . . . . . . . . . . . . . . . . 129

Loïc Chalmel: Pour une épistémologie de l'histoire des idées pédagogiques 141

\section{La psychologie}

Claudine Blanchard-LAville: Du côté des recherches cliniques d'orientation psychanalytique en sciences de l'éducation. . . . . . . . . . . . . . 153

\section{La philosophie}

Jean Houssaye: De la naissance des philosophes de l'éducation en France. . 165

Didier Moreau: De la recherche en philosophie de l'éducation 
— N 1967, les «sciences de l'éducation» étaient créées comme _ discipline universitaire en particulier à Caen sous l'impulsion de Gaston Mialaret. Le colloque organisé 40 ans plus tard se donnait pour projet de faire le point sur un certain nombre de questions institutionnelles et épistémologiques sur la discipline:

- Histoire, sociologie, psychologie et philosophie de l'éducation: fait-on de la recherche de la même façon dans les disciplines éponymes de référence et dans les sciences de l'éducation?

- Dans quelle mesure et selon quelles modalités les sciences de l'éducation ont-elles été amenées à définir de nouveaux territoires? Ont-elles suscité de nouvelles problématiques de recherche, accompagné de nouvelles demandes sociales?

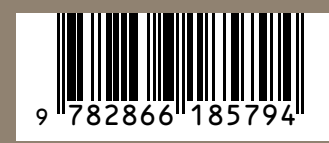

